\title{
LARGE-DISPLACEMENT LIGHTWEIGHT ARMOR
}

\author{
A Thesis \\ presented to \\ the Faculty of California Polytechnic State University,
}

San Luis Obispo

In Partial Fulfillment

of the Requirements for the Degree

Master of Science in Mechanical Engineering

by

Eric Christopher Clough

December 2013 
(C) 2013

Eric Christopher Clough

ALL RIGHTS RESERVED 


\section{COMMITTEE MEMBERSHIP}

TITLE:

AUTHOR:

DATE SUBMITTED:

COMMITTEE CHAIR:

COMMITTEE MEMBER
Large-displacement Lightweight Armor

Eric Christopher Clough

December 2013
Tom Mackin, $\mathrm{PhD}$

Professor of Mechanical Engineering

COMMITTEE MEMBER: Jacob Hundley, PhD

Member of the Technical Staff, HRL Laboratories 


\begin{abstract}
Large-displacement Lightweight Armor

Randomly entangled fibers forming loosely bound nonwoven structures are evaluated for use in lightweight armor applications. These materials sacrifice volumetric efficiency in order to realize a reduction in mass versus traditional armor materials, while maintaining equivalent ballistic performance. The primary material characterized, polyester fiberfill, is shown to have improved ballistic performance over control samples of monolithic polyester as well as 1095 steel sheets. The response of fiberfill is investigated at a variety of strain rates, from quasistatic to ballistic, under compression, tension, and shear deformation to elucidate mechanisms at work during ballistic defeat. Fiberfill's primary mechanisms during loading are fiber reorientation, fiber unfurling, and frictional sliding. Frictional sliding, coupled with high macroscopic strain to failure, is thought to be the source of the high specific ballistic performance in fiberfill materials. The proposed armor is tested for penetration resistance against spherical and cylindrical $7.62 \mathrm{~mm}$ projectiles fired from a gas gun. A constitutive model incorporating the relevant deformation mechanisms of texture evolution and progressive damage is developed and implemented in Abaqus explicit in order to expedite further research on ballistic nonwoven fabrics.
\end{abstract}




\section{Acknowledgments}

I would like to first thank my advisor, Professor Mackin, for the good talks about engineering, science, and philosophy. Without your input and direction none of this thesis would have ever come together. I also want to acknowledge Professor Mase for his input and guidance on the modeling and continuum mechanics sections. I want to thank Jake Hundley for taking time out of his busy schedule to teach me the ins and outs of user subroutines and damage mechanics, and for the many discussions on mechanics. I want to acknowledge Thijs van Loon and all of the hard work and enthusiasm that he put in to running experiments, not to mention all of the excellent Matlab scripts he contributed, all of the Subway sandwich breaks he forced me to take, and all of the great insights he had on the project.

A huge thanks to the folks at UCSB: Oshin Nazarian, Pete Maxwell, Kirk Fields, and Professor Frank Zok for the tremendous amount of help and guidance in completing ballistic experiments on their light gas gun.

I want to thank the entire grad-lab crew. Your late night/early morning antics, and dedication to working hard, but still having fun went a tremendous way in keeping my spirits up throughout the course of this thesis.

Thanks to everyone that helped in some way to get all of the equipment, software, and test fixtures I needed to use up and running. Thank you Josh Smith and Dustin Draper for your help in machining test fixtures, and thanks to Nash Anderson for the SEM work.

Most importantly, I want to thank my family for teaching me my love of science, engineering, and hard work. Thank you for supporting me throughout my life. None of this would be possible without you. 


\section{Table of Contents}

List of Tables ............................................................................................................. ix

List of Figures........................................................................................................................................ $\mathrm{x}$

1 Introduction ....................................................................................................................... 1

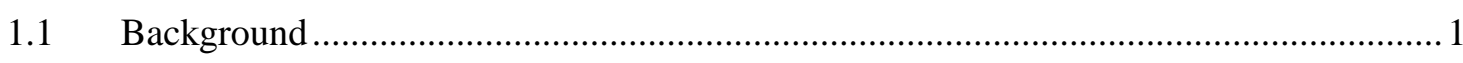

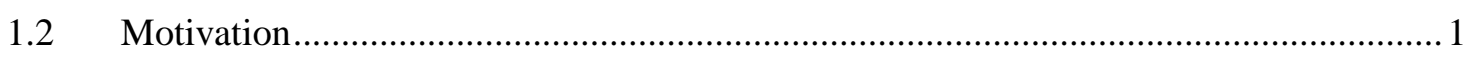

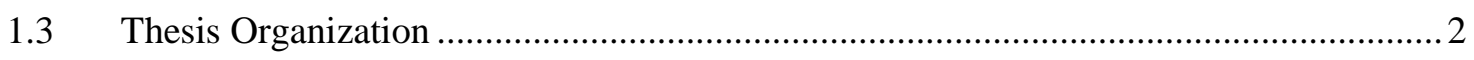

2 Armor Materials and Defeat Mechanisms .................................................................... 4

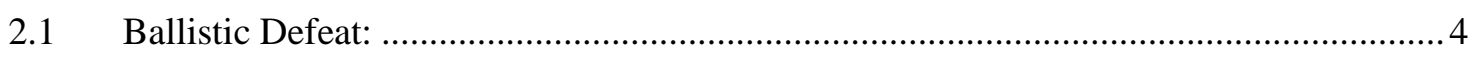

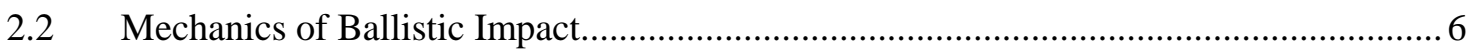

2.3 Monolithic Armor Plates................................................................................... 7

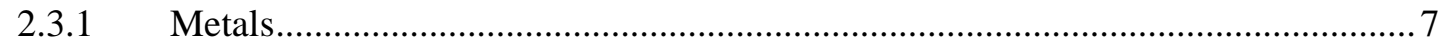

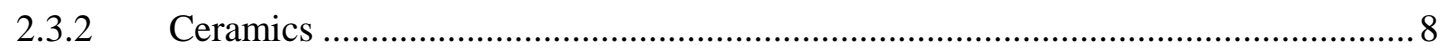

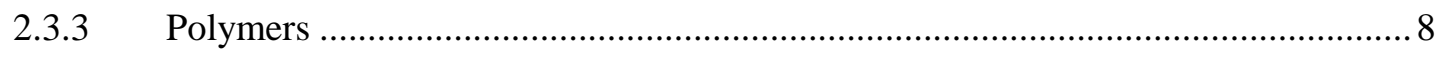

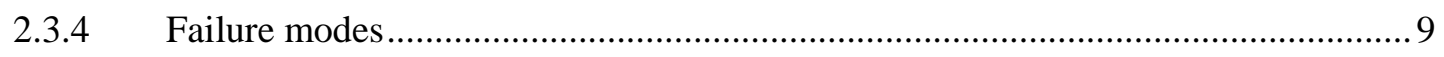

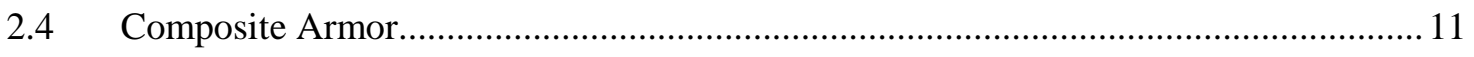

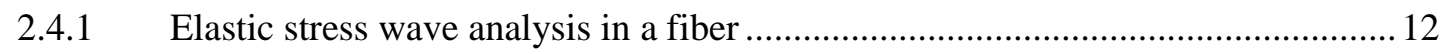

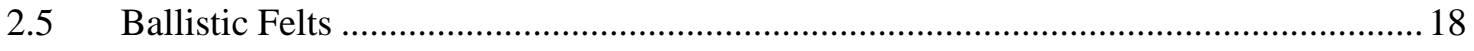

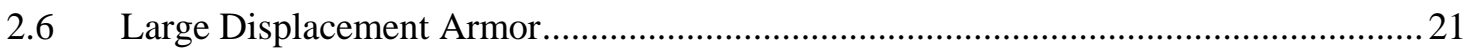

$3 \quad$ Preliminary Ballistic Testing ........................................................................................... 23

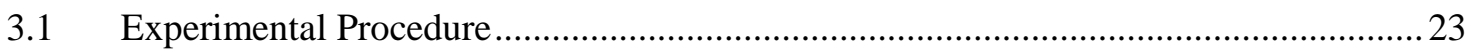




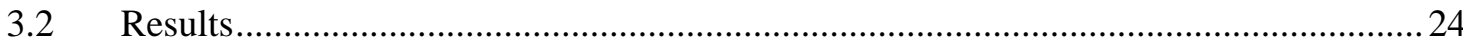

4 Mechanical Testing and Micromechanics ........................................................ 28

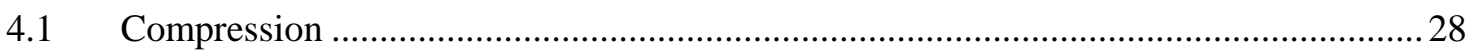

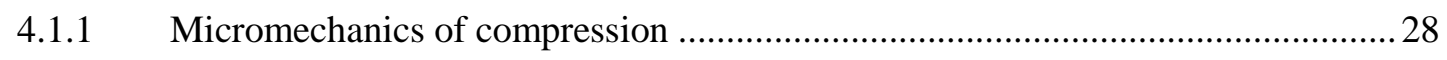

4.1.2 Compression experimental setup and procedure..............................................29

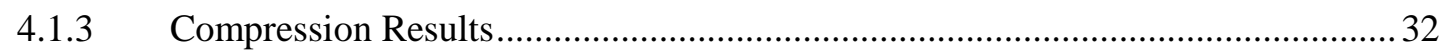

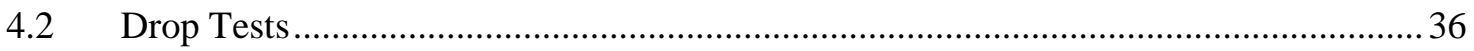

4.2.1 Micromechanics of drop test experiments ........................................................ 36

4.2.2 Drop test experimental setup and procedure ..................................................... 36

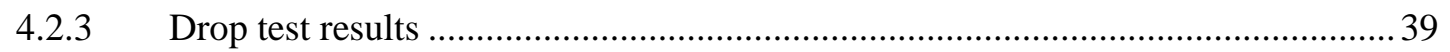

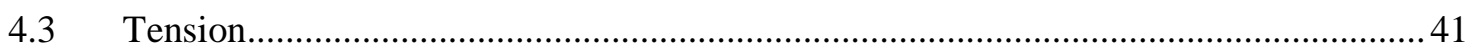

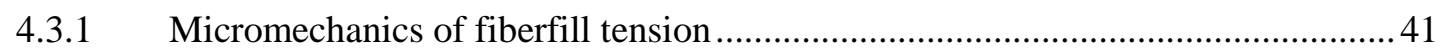

4.3.2 Tension experimental setup and procedure ................................................... 41

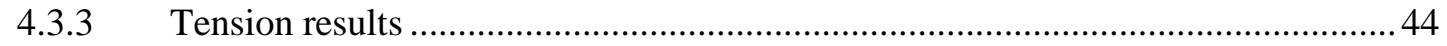

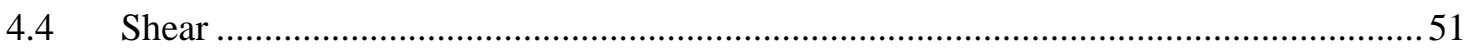

4.4.1 Micromechanics of fiberfill Shear ................................................................ 51

4.4.2 Shear experimental setup and procedure.........................................................51

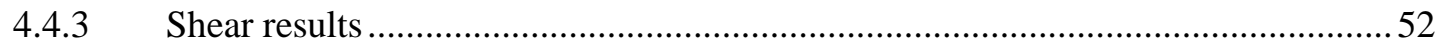

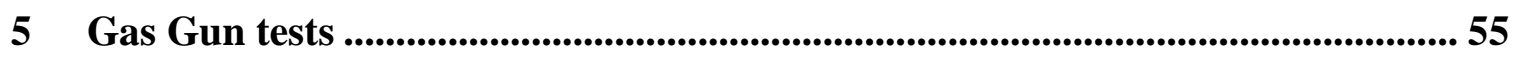

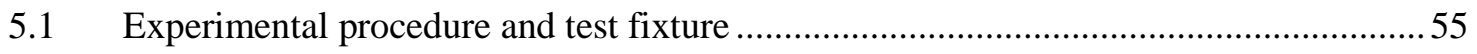

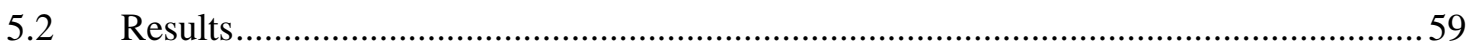


6 Numerical Analysis..................................................................................................... 69

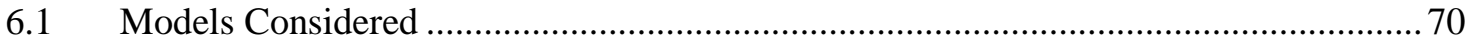

$6.2 \quad$ Fiberfill Continuum Model ...............................................................................

6.2.1 Progressive Damage Model ............................................................................73

6.2.2 Abaqus implementation ............................................................................

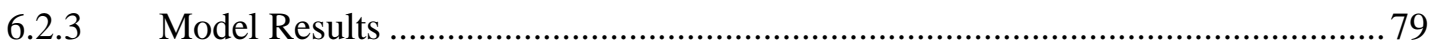

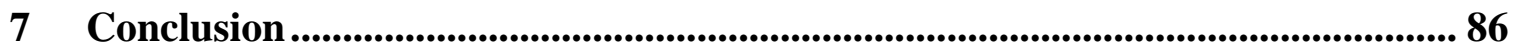

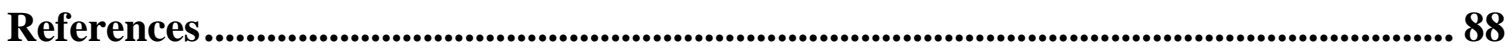

Appendix A. Complete drop test results ................................................................... 100

Appendix B. JS Model Derivation ............................................................................ 103

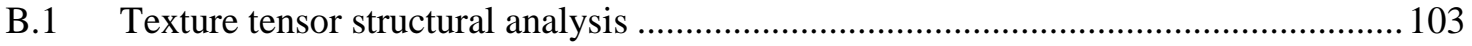

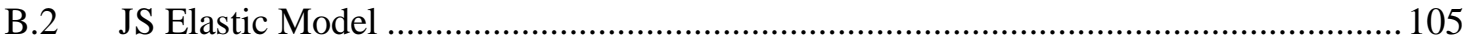

B.3 Inelastic extension of the JS model......................................................................... 108

Appendix C. JS VUMAT FORTRAN Code ......................................................................... 118 


\section{List of Tables}

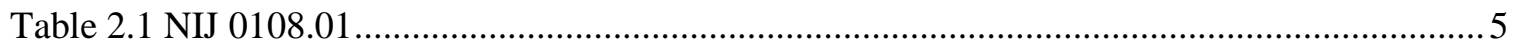

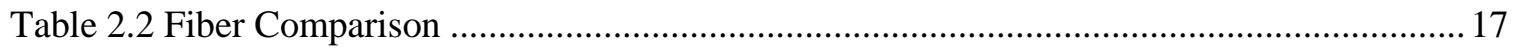

Table 2.3 Areal density comparison of Nylon ballistic felt with metals of equivalent ballistic

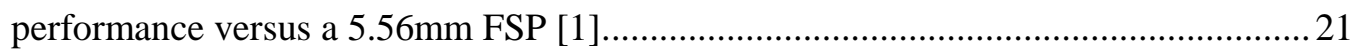

Table 3.1 Ammunition details (Manufacturer's Data) .................................................................. 23

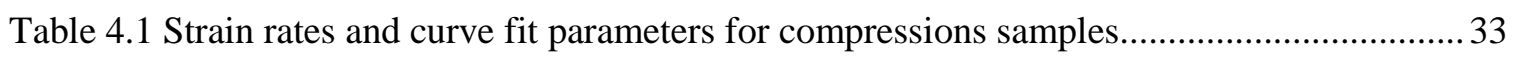

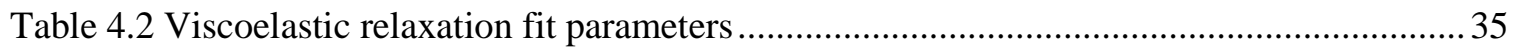

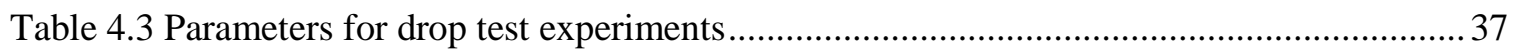

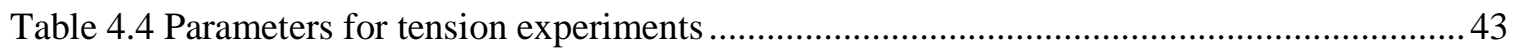

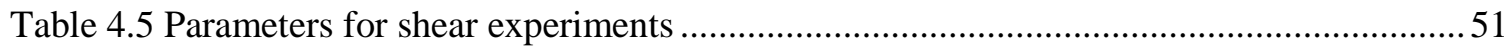

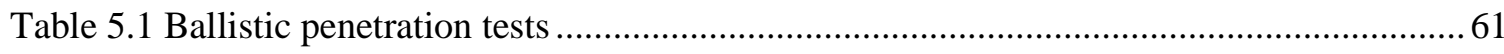

Table 6.1 Parameters used in model results (unless otherwise noted) …...................................79 


\section{List of Figures}

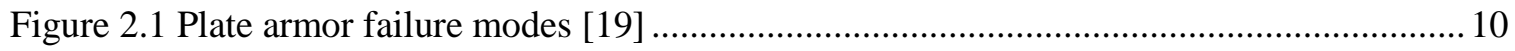

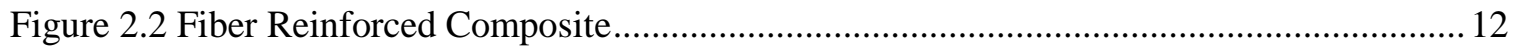

Figure 2.3 Longitudinal dynamic deformation of a single fiber. ............................................ 13

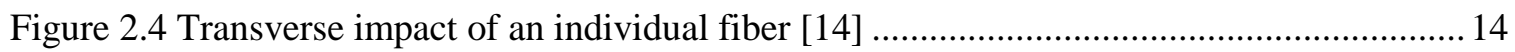

Figure 2.5 Linear-Log plot of strain energy behind the elastic wave front versus specific fiber

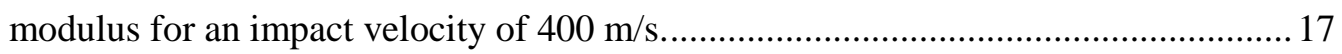

Figure 2.6 Fiber ballistic properties. Contours of constant $\Phi$ c shown [33] to [38]. ..................... 18

Figure 3.1 Schematic of the second ballistic experimental setup. ............................................24

Figure 3.2 "Cocoon" structures surrounding defeated projectiles.............................................25

Figure 3.3 Defeated projectile being extracted from a "cocoon." The tail on this projectile has

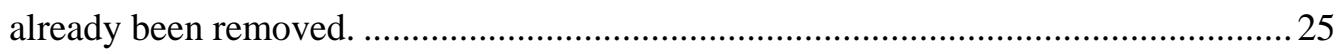

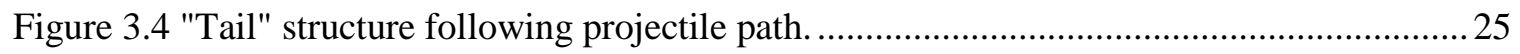

Figure 3.5 "Tail" emanating from the side of a defeated projectile............................................25

Figure 3.6 Fibers from the projectile affected region showing melting and adhesion...................26

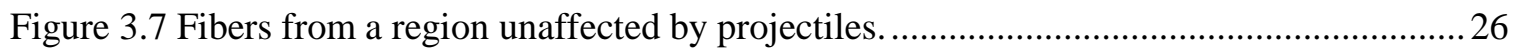

Figure 3.8 Close up of fiber from the affected region showing inter-fiber friction wear and

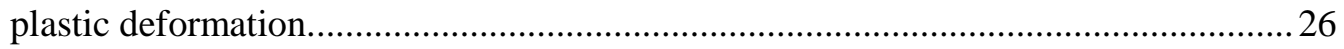

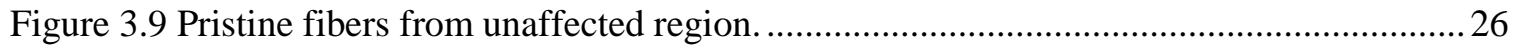

Figure 3.10 Results of the exit velocity experiments, values shown are averages of multiple

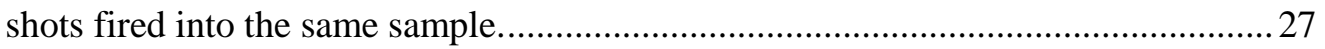

Figure 4.1 Compression test fixture mounted to servo hydraulic test frame …............................ 30

Figure 4.2 Example of toe correction procedure for compression data ..................................... 31

Figure 4.3 Compressive stress versus volume fraction (all data shown). Fit to the Toll van-

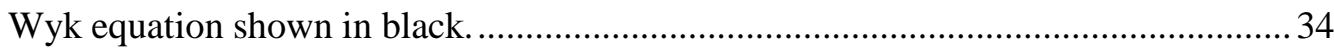


Figure 4.4 Compressive stress versus true strain. Fit to the Meziex form of the Toll van-Wyk

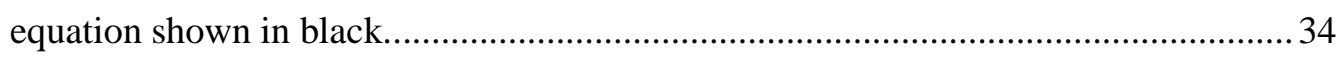

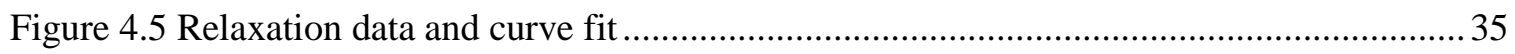

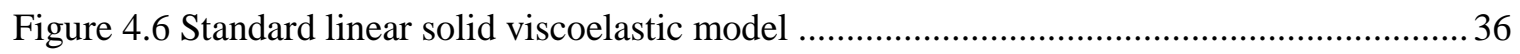

Figure 4.7 Drop test fixture mounted in the Dynatup drop tower............................................... 38

Figure 4.8 High-speed camera used in drop test experiments. .................................................. 38

Figure 4.9 Stress versus Nominal Strain curves for differing cross head speeds, Density =

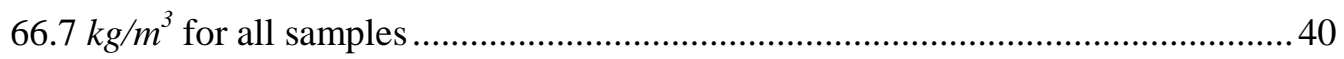

Figure 4.10 Fiberfill sample preparation........................................................................ 42

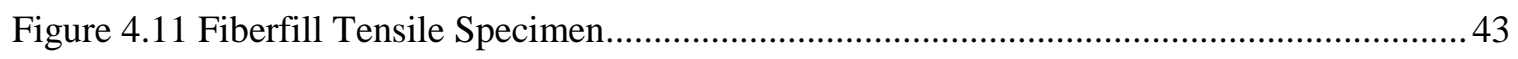

Figure 4.12 Images from tensile test 007. See Figure 4.13 ................................................... 45

Figure 4.13 Tensile test 007 ; several load-unload cycles shown .............................................. 46

Figure 4.14 Tensile data; low density samples ................................................................. 47

Figure 4.15 Tensile data; low density samples, small strain regime ......................................... 47

Figure 4.16 Comparison of high (009), medium (008) and low (003) density tensile samples..... 48

Figure 4.17 Comparison of high (009), medium (008) and low (003) density tensile samples;

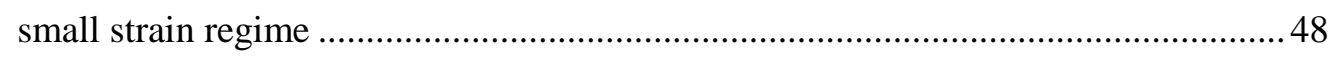

Figure 4.18 Peak stress versus volume fraction; cross direction samples. Power law fit shown in black

Figure 4.19 Initial modulus versus volume fraction; cross direction samples. Power law fit shown in black 49

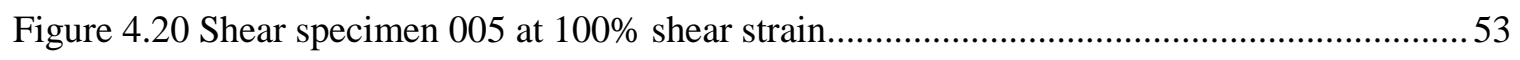

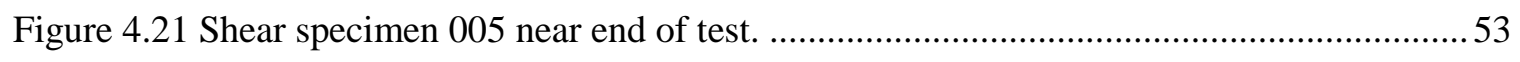

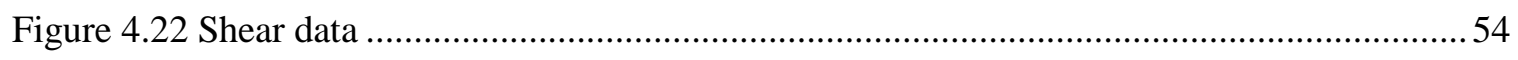

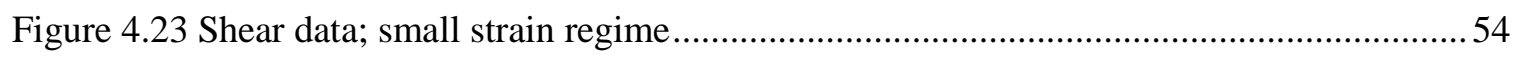

Figure 5.1 Gas gun configured with $45 \mathrm{~mm}$ barrel ........................................................... 58 
Figure 5.2 Gas gun schematic (not to scale)

Figure 5.3 Kevlar-PET layered fiberfill secured in test fixture .59

Figure 5.4 BB Tests; comparison of PET, Steel, and Fiberfill 61

Figure 5.5 Comparison of Test ID $004\left(10.39\left[\mathrm{~kg} / \mathrm{m}^{2}\right]\right)$ and Test ID $009\left(8.83\left[\mathrm{~kg} / \mathrm{m}^{2}\right]\right)$. 62

Figure 5.6 Test ID 010; low density Fiberloft vs BB projectile. 62

Figure 5.7 Test ID 011 and Test ID 012; Fiberloft vs FSPs 63

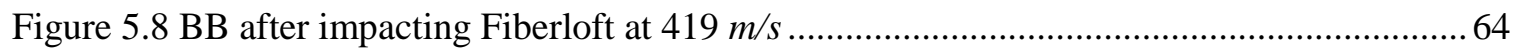

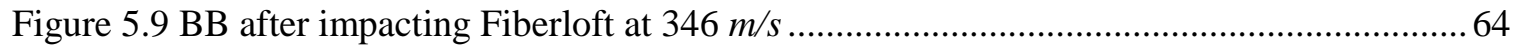

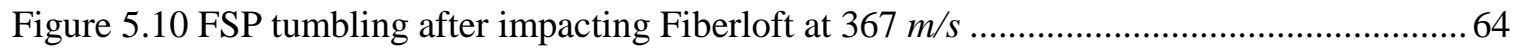

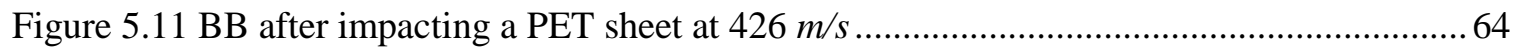

Figure 5.12 PET specimen showing cone and radial cracking after being impacted at $293 \mathrm{~m} / \mathrm{s} \ldots 65$

Figure 5.13 Kevlar/PET fiberfill enveloping a defeated projectile .66

Figure 5.14 Projectile stopped in a Kevlar tail. 66

Figure 5.15 Defeated projectile with foam obturator still intact 67

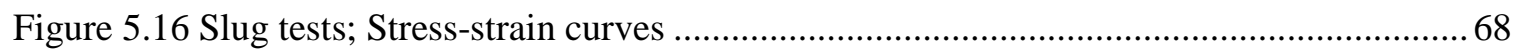

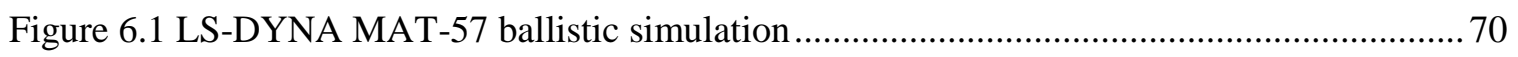

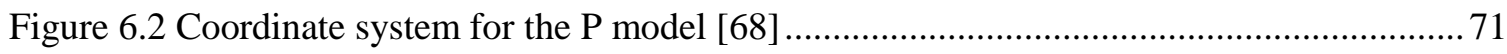

Figure 6.3 Illustration of the concept of effective area. .......................................................... 75

Figure 6.4 Boundary conditions and geometry for tension simulation ...................................... 81

Figure 6.5 JS model tension results; low strain regime (model results shown in black) .............. 81

Figure 6.6 JS model tension results (model results shown in black) …..................................... 82

Figure 6.7 Effect of changing the damage viscosity parameter .............................................. 82

Figure 6.8 JS model with and without progressive damage..................................................... 83

Figure 6.9 Damage localization in tension simulation (contours of damage shown) ................... 83

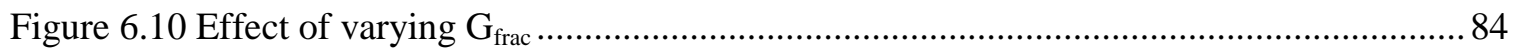

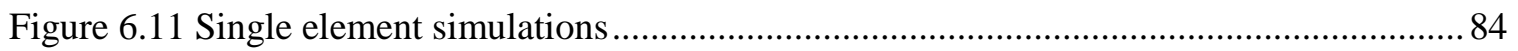




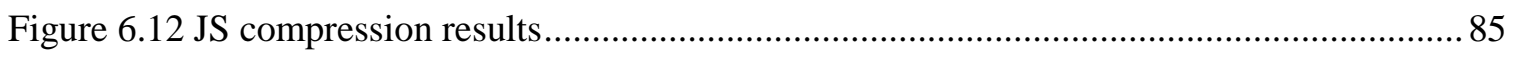

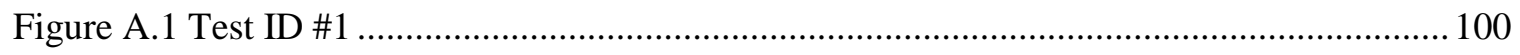

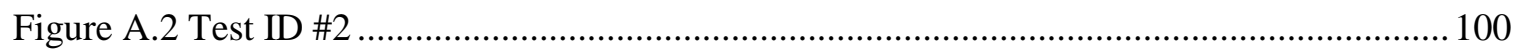

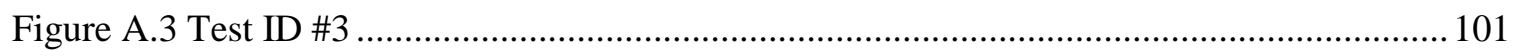

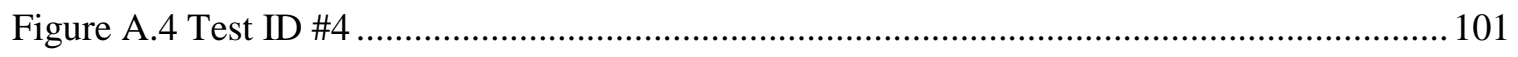

Figure A.5 Comparison of Test ID \#1 and Test ID \#2 fast impact; high versus low density ...... 101

Figure A.6 Comparison Test ID \#3 and Test ID \#4 slow impact; high versus low density......... 102

Figure A.7 Comparison of Test ID \#1 and Test ID \#3 high density; fast versus slow impact .... 102

Figure A.8 Comparison of Test ID \#2 and Test ID \#4 low density; fast versus slow impact...... 102

Figure B.1 Kroner-Lee Split of the deformation gradient [70] ............................................... 109 


\section{Introduction}

\subsection{Background}

The need for improved protection against ever increasing threat levels has evolved throughout history. Man has utilized nearly every class of material in search of protection from would-be threats. Starting from natural materials such as wood or dried animal hides and progressing into the $21^{\text {st }}$ century with complex composite armor systems utilizing technical ceramics, high strength fibers, nanoparticle filled matrices, and, in the case of armored vehicles, explosive reactive components. The need for lightness goes hand-in-hand with the need for sufficient protection when it comes to battlefield effectiveness. Mobility is key to survival and longevity in the field of battle. However, without sufficient protection high mobility is of little use. Necessarily, armor designers must make tradeoffs between these two attributes in order to optimize any armor system.

The need for lightness is nowhere more pronounced than in lightweight tactical vehicles, which are designed to be quick and maneuverable, and are ideally transportable via airlift. Often lightweight tactical vehicles are sparingly armored, relying heavily on active avoidance of enemy fire. This can be to the detriment of passengers, cargo, or equipment in these vehicles, as relatively low threat level munitions can easily cause loss of function or loss of life. There exists a need for lightweight armor that can protect against low level ballistic threats without significantly affecting total vehicle mass.

\subsection{Motivation}

This project began with the goal to develop an armor system for a robotic vehicle used in live-fire military training exercises. The principal munition used in these exercises is $5.56 \times 45 \mathrm{~mm}$ M855 NATO ball round. It was determined that to meet the protection and multi-hit performance required for this application, the majority of the robotic platform's load carrying capacity would 
have to be dedicated to ballistic armor. An armor system was devised that, rather than sacrificing payload capacity, sacrificed volumetric efficiency to obtain the ballistic performance required for the application.

\subsection{Thesis Organization}

This thesis aims to keep the application of large-displacement lightweight vehicular armor close at hand, and attempts to relate all experimental and theoretical work back to this end application. This thesis is organized as follows. Chapter 2 motivates the direction taken in researching large displacement armors by beginning with a discussion of ballistic defeat, the mechanisms associated with ballistic defeat, and the materials used in state-of-the-art armor. Chapter 2 ends with a discussion on ballistic non-woven felts, and emphasizes the mechanisms particular to this type of armor. A nonwoven material, polyester fiberfill, which possesses the desirable characteristics of a ballistic felt, namely: high fiber mobility and low density, is evaluated for ballistic performance in Chapter 3. Results from the somewhat crude proof-of-concept tests conducted in Chapter 3 serve to inform the remainder of the experimental study of large displacement armor; first in the quasi-static and low rate dynamic regime (Chapter 4) and then in the ballistic regime (Chapter 5). Chapter 4 includes a short review section on mechanisms and prior research for each of the relevant deformation modes: compression, tension, and shear, and includes comparisons of experimental observations with predictions from the micromechanical theory of entangled fiber networks. Chapter 5 chronicles ballistic testing conducted using a light gas gun and compares fiberfill armor performance and mechanisms with equivalent areal density steel and polyester sheets. Ballistic tests are a time consuming activity, and a more efficient means of studying variations of the parameter space associated with large-displacement lightweight armor systems is needed to further research in this field. To this end, in Chapter 6, a numerical model is sought that can capture the relevant physics of non-woven deformation under arbitrary loading conditions. It was discovered that no suitable material model was available in 
either of the commercial finite element analysis programs considered. Therefore, a material model that was capable of simulating fiberfill deformation behavior was developed and implemented in Abaqus explicit utilizing a VUMAT subroutine. Chapter 7 summarizes the work contained in this thesis and discusses future applications of large-displacement armor. 


\section{Armor Materials and Defeat Mechanisms}

\subsection{Ballistic Defeat:}

The basic science of ballistic defeat is simple; however, the various mechanisms involved are complex. A projectile is stopped when it is robbed of its kinetic energy by an object acting to oppose the projectile's motion. This can be described by the relation [1]:

$$
\int \mathrm{Fdx}=\frac{1}{2} \mathrm{mv}_{0}^{2}
$$

The left hand side of this equation describes the work done by the retarding force $\mathrm{F}$ through the deflection distance $\mathrm{dx}$, and the right hand side of the equation describes the kinetic energy of the projectile of mass $\mathrm{m}$ with an initial velocity of $\mathrm{v}_{0}$. This can also be cast in the impulse-momentum form of Newton's second law of motion [1]:

$$
\int \mathrm{Fdt}=\int \mathrm{mdv}
$$

From these two relations it is clear that either large forces or large deflections and contact times must arise in the process of ballistic defeat in order to stop a projectile with significant momentum/kinetic energy. Forces give rise to stresses in both the projectile and the material acting to stop the projectile. Describing these stresses accurately via appropriate rate-dependent constitutive laws is where theoretical analysis becomes complicated. The effects of various mechanisms for energy dissipation must be accounted for in these constitutive laws such as internal friction, formation of new surfaces via fracturing, or phase changes within the material. Further complicating matters, is the transport of stress waves radiating from the impact site and interacting with boundaries and other stress waves in a complex manner.

The purpose of armor is to harness these mechanisms, both elastic and inelastic, to defeat a projectile over a given distance in order to protect what lies beyond that distance. The 
mechanisms utilized in armor materials vary depending on the particular application. A great deal of research has been conducted to elucidate the relationship between failure mechanisms and material properties during ballistic impact, but no uniform design methodology has emerged [2] to [14]. This is a direct consequence of the broad range of applications of armor systems each with different design constraints with respect to threat level, area density $\left(A_{d}=\frac{\text { mass }}{\text { area }}\right)$, thickness, dynamic deflection, and multi-hit performance [15].

Ballistic armor levels are classified based on the type of threat they are designed to withstand. The National Institute of Justice (NIJ) ranks armors (except for police body armor and ballistic helmets) according to NIJ 0108.01, and requires that an armor specimen tolerate 5 hits by the projectile listed without any penetrations, except for threat level IV which only requires 1 hit to be stopped. These threat levels are summarized in Table 2.1. Other standards for armor include: NIJ 0101.04, NIJ 0106.01, ISO14876, NATO STANAG 4569, and MIL-STD-662F.

Table 2.1 NIJ 0108.01

\begin{tabular}{|c|c|c|c|c|}
\hline $\begin{array}{l}\text { Protection } \\
\text { Level }\end{array}$ & Ammunition & $\begin{array}{c}\text { Mass } \\
{[\mathrm{g}]}\end{array}$ & $\begin{array}{l}\text { Suggested Barrel Length } \\
\qquad[\mathrm{cm}]\end{array}$ & $\begin{array}{l}\text { Projectile velocity } \\
{[\mathrm{m} / \mathrm{s}]}\end{array}$ \\
\hline \multirow{2}{*}{ I } & $.22 \mathrm{LRHV}$ & 2.6 & \multirow{2}{*}{15 to 16.5} & $320 \pm 12$ \\
\hline & .38 Special RN & 10.2 & & $259 \pm 15$ \\
\hline \multirow{2}{*}{ II-A } & .357 Mag JSP & 10.2 & \multirow{2}{*}{10 to 12} & $381 \pm 15$ \\
\hline & 9mm FMJ & 8.0 & & $332 \pm 12$ \\
\hline \multirow{2}{*}{ II } & .357 Mag JSP & 10.2 & 15 to 16.5 & $425 \pm 15$ \\
\hline & $9 \mathrm{~mm}$ FMJ & 8.0 & 10 to 12 & $358 \pm 12$ \\
\hline \multirow{2}{*}{ III-A } & .44 Mag SWC & 15.55 & 14 to 16 & \multirow{2}{*}{$426 \pm 15$} \\
\hline & 9mm FMJ & 8.0 & 24 to 26 & \\
\hline \multirow[b]{2}{*}{ III } & $7.62 \mathrm{~mm}$ & \multirow[b]{2}{*}{9.7} & \multirow[b]{2}{*}{56} & \multirow[b]{2}{*}{$838 \pm 15$} \\
\hline & $\begin{array}{c}.308 \text { Winchester } \\
\text { FMJ }\end{array}$ & & & \\
\hline IV & $.30-06 \mathrm{AP}$ & 10.8 & 56 & $868 \pm 15$ \\
\hline
\end{tabular}

LRHV- Long Rifle High Velocity

RN- Round Nose

Mag- Magnum

FMJ- Full Metal Jacket

JSP- Jacketed Soft Point

SWC-Semi Wad Cutter

AP- Armor Piercing 
The velocities in the NIJ threat levels are $\mathrm{V}_{0}$ velocities, defined as the velocity at which there is a $0 \%$ chance of a projectile penetrating the given armor. As it is very difficult to determine $\mathrm{V}_{0}$ directly by experiment, $\mathrm{V}_{50}$ is a more common armor metric. $\mathrm{V}_{50}$ represents the velocity at which there is a 50\% probability that armor will stop a particular threat, and is discussed in MIL-STD662-F. There are different interpretations depending on the standard of what counts as a defeat of a particular armor. This work will adopt the definition of MIL-STD-662-F, in which the ballistic limit is defined based on penetration of a witness sheet placed a specified distance behind the target from the armor.

\subsection{Mechanics of Ballistic Impact}

Elastic properties pertinent to ballistic defeat are [5] [6]:

Elastic wave speed:

Acoustic impedance:

$$
\mathrm{C}=\sqrt{\frac{\mathrm{E}}{\rho}}\left[\frac{m}{s}\right]
$$

Specific energy absorption:

$$
\mathrm{Z}=\sqrt{\mathrm{E} \rho}\left[\frac{N-s}{m^{3}}\right]
$$

$$
\mathrm{U}=\frac{\sigma_{\mathrm{y}} \varepsilon_{\mathrm{y}}}{2 \rho}\left[\frac{P a-m^{3}}{k g}\right]
$$

Where $\mathrm{E}$ is the modulus with units of pressure, $\rho$ is the mass density, and $\sigma_{\mathrm{s}}$ and $\varepsilon_{\mathrm{s}}$ are the strength and failure strain respectively. Elastic wave speed represents the transmission of stress away from the impact site, and thus correlates to the volume of material engaged in the ballistic defeat. Acoustic impedance relates to the local pressures built up at the impact site that serve to deform the incoming projectile, but consequently also tend to cause yielding or fracture in the armor. The specific energy absorption attempts to capture the amount of energy a material can 
store elastically and is a linear approximate of the area under the stress-strain curve normalized by the mass density. Other important material properties are fracture toughness $\mathrm{K}_{\mathrm{c}}$ and hardness $\mathrm{H}$, the former represents the ability of a material to resist crack growth and the latter represents a material's resistance to permanent local deformation. $\mathrm{Z}$ and $\mathrm{H}$ are key to projectile erosion, but without adequately high values of $\mathrm{C}$ and $\mathrm{U}$, sufficient projectile erosion and energy absorption cannot be achieved. Tradeoffs between these parameters and the concomitant change in ballistic performance are not well understood [5]. None of these properties is very useful in predicting ballistic performance unless their behavior can be determined in the high strain rate regimes characteristic of ballistic impact. Moreover, these material properties are merely an estimate of a given materials usefulness in an armor application, and ballistic performance is not directly defined by them. However, ballistic performance is coupled to these properties, and thus they are useful in providing a starting point for materials selection in armor applications.

Depending on the application and threat level, different materials are called for in the construction of an appropriate armor. Low mass is generally desirable for vehicle and personnel armor, but becomes less important for static structures. Personnel armor must be highly deformable and low profile so that it does not impair the armor wearer's mobility, but ground vehicle armor does not necessarily require these traits. Relevant armor materials fall into broad categories of metals, ceramics, polymers, and fiber reinforced composites. While there are many commonalities in the elastic regime, these materials differ greatly in their inelastic deformation characteristics.

\subsection{Monolithic Armor Plates}

\subsubsection{Metals}

Metallic armors are highly effective against kinetic energy projectiles, and offer good structural performance in addition to having desirable armor characteristics. Metals defeat projectiles 
through a combination of efficient elastic transmission of stress waves and plastic dissipation, and are some of the more common armor materials. Because of their ductility, metal armors have a high tolerance to multiple, non-penetrating, projectile impacts within a relatively small area, but suffer from being less mass efficient than high performance ceramic or composite armors. Monolithic metal armors are highly susceptible to High Explosive Anti-Tank (HEAT) munitions, which are designed to melt through armor plates using high energy jets of molten copper. Metallic armor capable of protecting against HEAT rounds must be very dense and very thick.

\subsubsection{Ceramics}

Ceramic armors are used in heavy armored vehicles and are used as trauma plates incorporated into composite personnel armor. Ceramics dissipate impact energy through deformation of projectiles due to high acoustic impedances, and efficient load spreading via high elastic wave speeds. Under compressive loads, ceramics exhibit ductile modes of energy dissipation by means of lattice plasticity and intergranular plasticity. Low ductility failure mechanisms of radial and cone cracking caused by microcracking within the ceramic also serve as dissipation mechanisms in ceramic armors. Comminution due to extensive cracking in a confined region has the added benefit of creating a high-energy spall of ceramic particles that erode the impacting projectile. The formation of high energy ceramic particles during comminution also serves to break up the molten copper jet from HEAT munitions and provides superior resistance to this type of ballistic threat. This comes at a cost, as multi-hit performance of ceramics is generally very poor due the extensive cracking that occurs in the vicinity of a projectile impact [5].

\subsubsection{Polymers}

Polymers are utilized extensively in transparent armor. Polymers can display ductile or brittle behavior depending on loading rate and temperature. Their dissipation mechanisms include polymer chain untangling, interchain slip, bond breakage, and fracture. They benefit from low densities and relatively high elongation to failure. 


\subsubsection{Failure modes}

Failure modes for armor plates composed of metals, ceramics, and polymers can be divided into two regimes: one of low ductility and one of high ductility [16] to [19]. Theoretical and experimental studies on these modes can be found in Backman [18] and Johnson [20], and a review on the matter can be found in Corbett [21].

In the low ductility regime spall fracture, plugging, and radial fracture are dominant failure modes. Spall fracture results from a compressive dilatational wave, formed at the impact site, reflecting as a tensile wave at the back of the plate with a magnitude sufficient to exceed the dynamic tensile strength of the material. Plugging can result either due to high shear stresses formed around the moving plug directly in front of the projectile or, in the case of ceramics, due to cone cracking. In the limit as projectile velocities increase, shear plugging often becomes the dominant failure mode. High velocity shear plugging occurs when a material has insufficient time to respond to an impact. Stresses ample enough to cause failure in the armor build up almost instantaneously at the impact site, with little stress wave propagation occurring prior to complete perforation of the armor, and a plug with approximately the same area as the projectiles frontal area is knocked out of the armor. Radial cracking occurs in materials with tensile strengths lower than their compressive strengths. As the initial compressive dilatational wave spreads outward, trailing radial tensile stresses lead to the formation of radial cracks emanating from the impact site.

In the high ductility regime petaling and ductile hole enlargement are the two failure mechanisms usually observed. Petaling, either frontal or rearward, is caused by high radial and circumferential tensile stresses after the pass of the initial dilatational wave. Rearward petaling is the result of bending moments created by strain of the material in the direction of the projectiles motion. As the material pushes ahead of the projectile, large plastic deformation occurs which leads to a starshaped pattern of petals initiating from a failure site near the point of impact. Plastic hinges form 
at the bases of the petals, and the petals then bend out of the way as the projectile passes them. Frontal petaling occurs in thicker sections of material and occurs due to a combination of unloading and projectile erosion opposite the direction of the projectiles path. Ductile hole enlargement is a common mechanism in thick plates impacted by conical or ogival tipped projectiles. At the contact site the projectile tip displaces material transversely, leading to a radial momentum that continues so that a hole in the plate is enlarged in the direction of the projectile's motion.

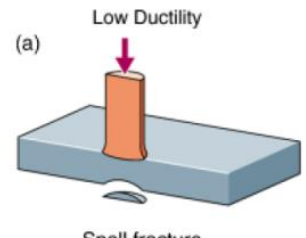

Spall fracture

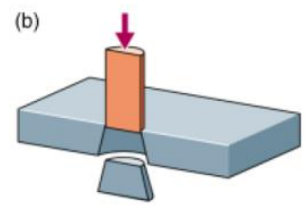

Plugging

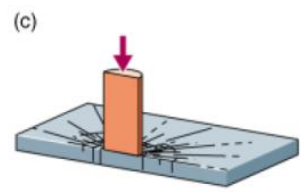

Radial fracture

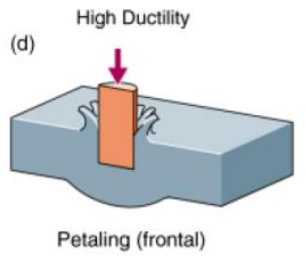

(e)

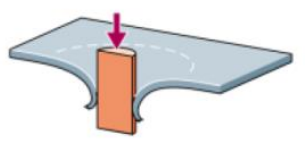

Petaling (rearward)

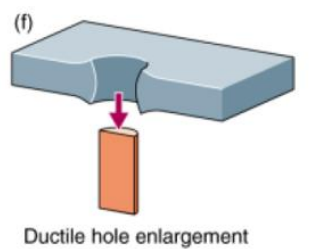

Figure 2.1 Plate armor failure modes [19]

Monolithic armor plates rely on a single material to provide sufficient ballistic resistance across a broad range of threat levels. One material may be better suited against a certain type of threat in one situation whereas another material type may be superior against a slightly different threat. For instance, a metallic armor may be better at stopping many small fragments impacting in a concentrated area, whereas a ceramic armor may be better at stopping a single high-hardness projectile. In practice, the ability to have both good multi-hit protection as well as high single-hit 
protection is needed. This often cannot be achieved using an armor composed of a single material.

\subsection{Composite Armor}

Composite armors can serve multifunctional structural and ballistic protection roles, and are composed of a myriad of constituents arranged in a systematic fashion. They fall into broad categories of fiber reinforced composites and laminated plate composites with significant overlap occurring between these two categories. Examples of composite armors include Composite Integral Armor (CIA) [22], [23]; Chobham armor, utilized on the M1 Abrams tank; Honeywell SpectraShield $^{\circledR}$; and Tencate Aramid-Shield ${ }^{\circledR}$.

In addition to the constituent material properties, parameters such as acoustic impedance mismatch and interfacial strength act in concert to dictate a composite armor system's performance [24]. Synergistic material behaviors can be harnessed in composite armor systems, which benefit from the splitting of roles between different constituents within the armor [25]. For example: bilayer armor composed of a ceramic outer layer and a metallic inner layer benefits from the ceramic's high hardness, which blunts or fractures oncoming projectiles and spreads the load on the inner layer, and the metallic layer's high ductility and damage tolerance. In CIAs, when an interlayer with low acoustic impedance is incorporated between the ceramic and supporting layers, an improvement in the overall armor system's performance can be realized without requiring an increase in $A_{d}$ [26] to [29].

Fiber reinforced composite armors represent the state of the art in ballistic protection. Fiber reinforced armors can be woven or non-woven, and are composed of fibers made from ceramics, metals, or polymers incorporated into a variety of matrix materials (Figure 2.2). Fibers possess high specific properties, and often exceed the mechanical performance of their parent material. 

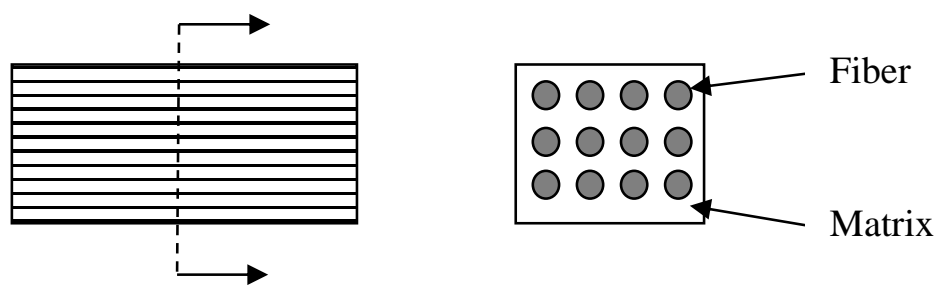

Figure 2.2 Fiber Reinforced Composite

During a ballistic impact, the fibers directly engaged by the projectile (primary fibers), bear the brunt of the impact. Longitudinal and transverse stress waves propagate faster along the axes of these primary fibers, and in 0/90 laminates this leads to a pyramidal shaped cone deformation. The size of the cone is an indication of how far the impact energy has spread and how much energy has been absorbed by the composite.

\subsubsection{Elastic stress wave analysis in a fiber}

The mechanical behavior of individual fibers subject to ballistic impact is studied in Smith [30] and extended to multiple intersecting fibers in Roylance [31], [32]. Smith uses a rate independent approximation to study the stress wave transmission behavior of a single fiber. First, the earlier equation for wave speed is altered to account for the variation in modulus with strain:

$$
C=\sqrt{\left(\frac{1}{\rho} \frac{\mathrm{d} \sigma(\varepsilon)}{\mathrm{d} \varepsilon}\right)}
$$

Now, consider a fiber fixed at one end with a velocity $\mathrm{V}$ instantaneously applied to its free end. After a time increment dt, the strain will have propagated into the fiber a distance Cdt while the free end will have displaced outward an amount Vdt (Figure 2.3). 


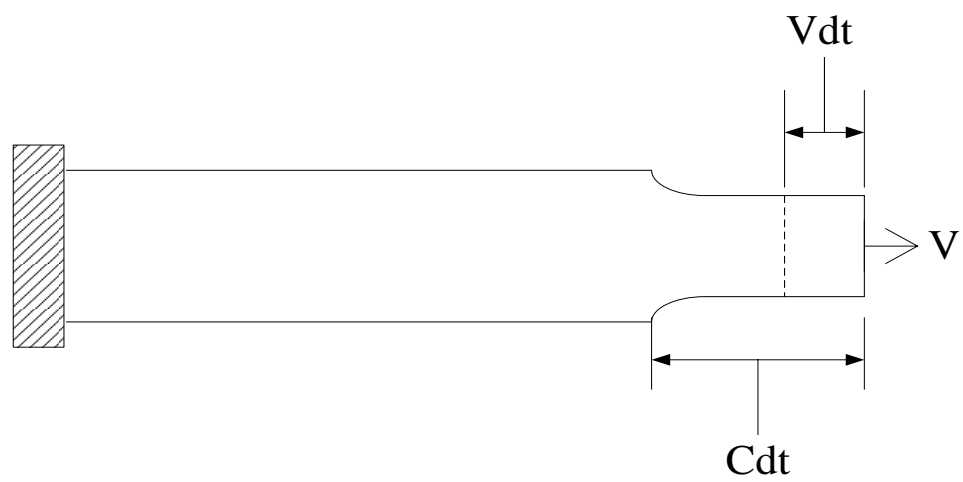

Figure 2.3 Longitudinal dynamic deformation of a single fiber.

The strain resulting from this deformation is then:

$$
\varepsilon=\frac{\mathrm{Vdt}}{\mathrm{Cdt}}=\frac{\mathrm{V}}{\mathrm{C}}
$$

and the stress is:

$$
\sigma=\frac{d \sigma}{d \varepsilon} \varepsilon=V \sqrt{\rho \frac{d \sigma}{d \varepsilon}}
$$

The shape of the wave front is dictated by the dynamic stress versus strain curve. If $\frac{\mathrm{d}^{2} \sigma}{\mathrm{d} \varepsilon^{2}}<0$ then each succeeding increment of strain in the propagating wave travels more slowly than the previous increment and the wave broadens as it travels. However, if $\frac{\mathrm{d}^{2} \sigma}{\mathrm{d} \varepsilon^{2}} \geq 0$ then each strain increment travels faster than the previous increment; this will lead to stacking up at the wave front and will form a shock wave. In general, a stress wave may contain both dispersive and shock components.

The velocity of a material point behind the wave is described by:

$$
\mathrm{w}=\int_{0}^{\varepsilon_{0}} \mathrm{Cd} \varepsilon
$$

Where $\varepsilon_{0}$ is the ultimate value of strain generated by the impact. Since w must equal V: 


$$
\mathrm{V}=\int_{0}^{\varepsilon_{0}} \mathrm{Cd} \varepsilon
$$

The transverse impact case, more pertinent to ballistic behavior, involves the propagation of longitudinal (with respect to the fiber axis) and transverse waves. Longitudinal waves radiate outward from the point of impact leading to particle velocities in the inward direction. Trailing the longitudinal wave front is a transverse "kink" wave.

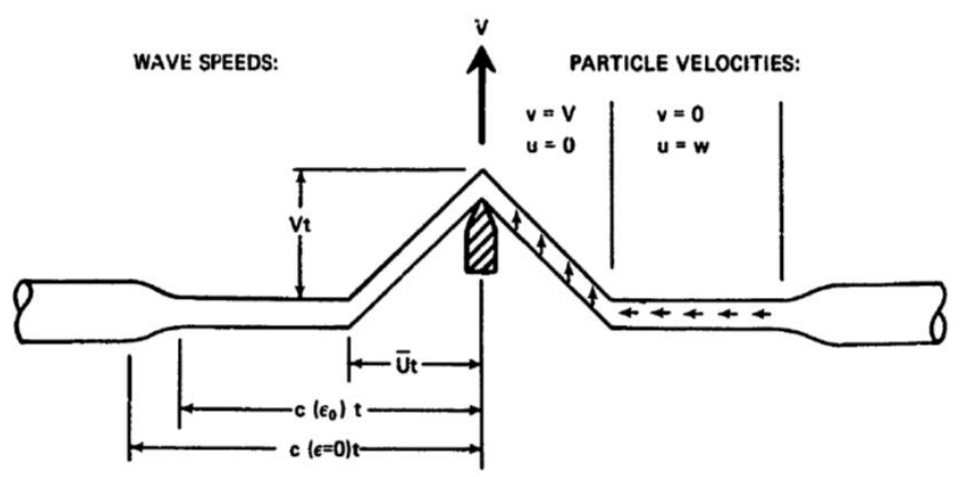

Figure 2.4 Transverse impact of an individual fiber [14]

At the kink wavefront, the particle velocity of the fiber abruptly changes to match the speed and direction of the impacting projectile. Tension and strain are continuous across the kink wavefront, but particle velocities are discontinuous. This is referred to in Roylance [31] as a geometrical shock. The seemingly unbalanced tensions on either side of the kink wave are compensated by the change in particle momentum as the wave propagates. Behind the kink wavefront all particle velocities are equal to the projectile velocity and the fiber forms a straight line with an inclination $\theta$ from the initial plane of the fiber. The radial inward particle velocity is found from Eq 2.9.

The velocity of the kink wave, with respect to a moving reference frame (Lagrangian) attached to the extending fiber, is:

$$
U=\sqrt{\frac{\sigma_{0}}{\rho\left(1+\varepsilon_{0}\right)}}
$$


In the reference frame of a stationary observer, the kink wave's velocity is:

$$
\overline{\mathrm{U}}=\left(1+\varepsilon_{0}\right) \mathrm{U}-\mathrm{w}
$$

These are related to the impact velocity V by:

$$
V=\sqrt{\left(1+\varepsilon_{0}\right)^{2} U^{2}-\bar{U}^{2}}
$$

For the case of non-linear material behavior this system of equations can be solved numerically. As a first estimate, however, we can assume linear elastic, rate independent behavior. These assumptions lead to the equation:

$$
\mathrm{V}=\sqrt{\varepsilon_{0} \frac{\mathrm{E}}{\rho}\left(2 \sqrt{\varepsilon_{0}\left(1+\varepsilon_{0}\right)}-\varepsilon_{0}\right)}
$$

which relates the strain developed by an impact velocity in terms of the fiber modulus and density.

Utilizing the expression:

$$
\Psi=\frac{1}{2} \sigma_{0} \epsilon_{0}=\frac{1}{2} \mathrm{E} \epsilon_{0}^{2}\left[\frac{J}{m^{3}}\right]
$$

for the strain energy stored within the fiber, a plot of strain energy behind the wave for a given impact velocity can be plotted versus the specific modulus (Figure 5). For a low specific modulus a larger amount of strain energy is stored behind the wave front. If the strain energy behind the wave exceeds the critical strain energy:

$$
\Psi=\mathrm{E} \epsilon_{\mathrm{s}}^{2}=\Psi_{\mathrm{c}}
$$

where $\epsilon_{\mathrm{s}}$ is the failure strain of the fiber, the fiber ruptures, and ceases to spread elastic energy away from the impact. The Young's modulus cannot be increased indefinitely, however, without 
a reduction in the failure strain. This creates an optimization problem wherein an armor designer must select a fiber with sufficient modulus to spread energy to as much material as possible, but without significantly sacrificing the strain to failure.

From this analysis of individual fibers it can be shown that there exists an impact velocity, for a fiber with a given stress-strain behavior, at which a fiber reaches its critical strain energy instantaneously upon impact. This velocity, deemed the critical velocity for a given fiber, can be related back to the parameters proposed by Cuniff. The critical velocity of a given fiber can be estimated by [6], [33]:

$$
\Phi_{\mathrm{c}}=\sqrt[3]{\mathrm{CU}}\left[\frac{m}{s}\right]
$$

The values of $\Phi_{c}$ for some common fibers are listed in Table 2.2. This analysis does not take into account the interaction of stress waves with boundaries, fiber crossover points (as in woven composites), or with other stress waves. It also ignores rate dependence and the effects of inelastic deformation. By assuming that the quasistatic properties apply in the ballistic regime, erroneous results are obtained. For instance, carbon fiber is expected to have a similar ballistic performance to Spectra Fiber (Figure 2.6). In practice, carbon fiber is relatively ineffective in armor applications [13]. 


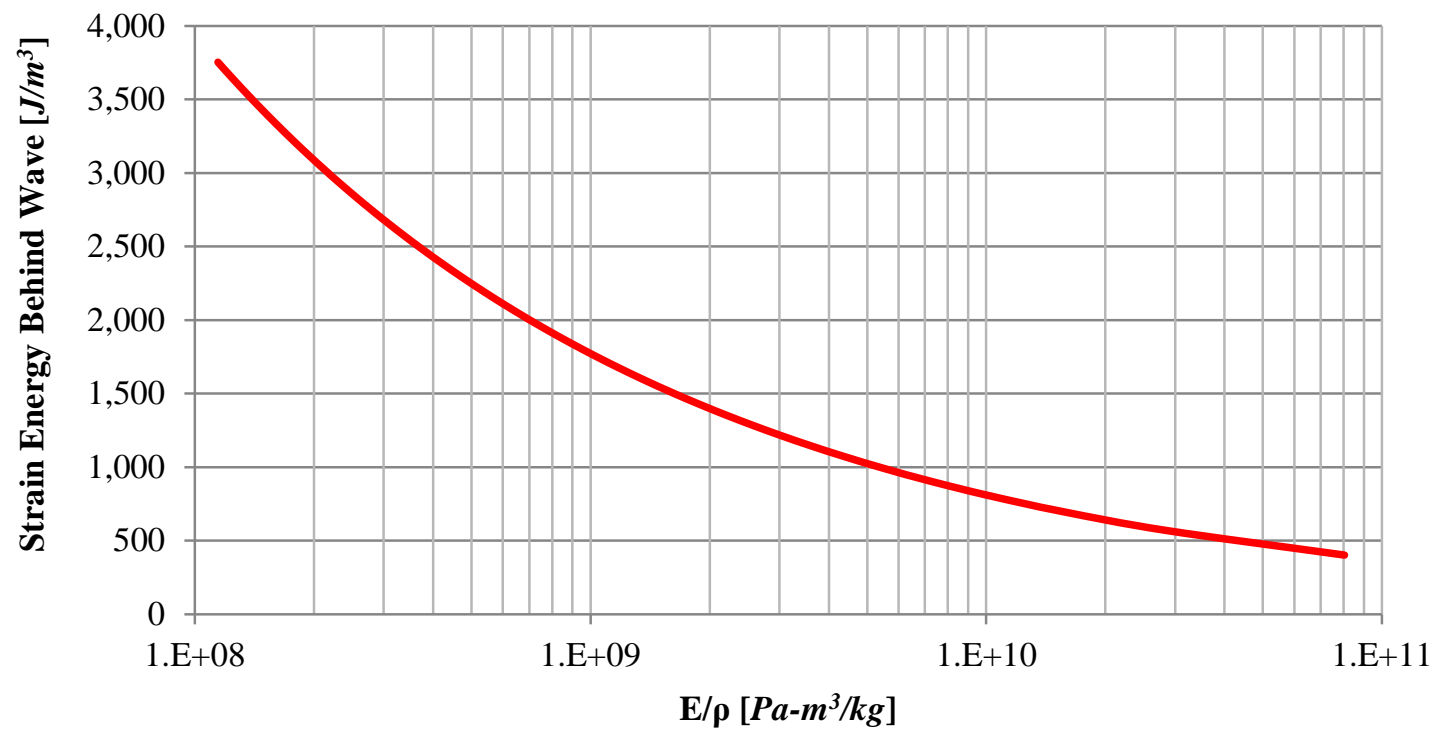

Figure 2.5 Linear-Log plot of strain energy behind the elastic wave front versus specific fiber modulus for an impact velocity of $400 \mathrm{~m} / \mathrm{s}$.

Table 2.2 Fiber Comparison

\begin{tabular}{|l|c|c|c|}
\hline Fiber Material & $\mathrm{C}\left[\frac{\mathrm{m}}{\mathrm{s}}\right]$ & $\mathrm{U}\left[\frac{\mathrm{J}-\mathrm{m}^{3}}{\mathrm{~kg}}\right]$ & $\Phi_{\mathrm{c}}\left[\frac{\mathrm{m}}{\mathrm{s}}\right]$ \\
\hline PET & $1.87 \mathrm{E}+03$ & $8.23 \mathrm{E}+04$ & 536 \\
\hline Nylon 6,6 & $2.88 \mathrm{E}+03$ & $7.91 \mathrm{E}+04$ & 611 \\
\hline Kevlar 29 & $7.00 \mathrm{E}+03$ & $3.65 \mathrm{E}+04$ & 634 \\
\hline Kevlar 49 & $8.83 \mathrm{E}+03$ & $2.50 \mathrm{E}+04$ & 604 \\
\hline Spectra 900 & $8.97 \mathrm{E}+03$ & $5.05 \mathrm{E}+04$ & 768 \\
\hline Spectra 1000 & $1.17 \mathrm{E}+04$ & $5.50 \mathrm{E}+04$ & 864 \\
\hline Spectra 2000 & $1.13 \mathrm{E}+04$ & $5.16 \mathrm{E}+04$ & 836 \\
\hline IM7 Carbon & $1.25 \mathrm{E}+04$ & $3.02 \mathrm{E}+04$ & 722 \\
\hline IM10 Carbon & $1.32 \mathrm{E}+04$ & $3.89 \mathrm{E}+04$ & 800 \\
\hline M5 & $1.33 \mathrm{E}+04$ & $6.25 \mathrm{E}+04$ & 940 \\
\hline S2 Glass & $5.94 \mathrm{E}+03$ & $5.67 \mathrm{E}+04$ & 696 \\
\hline
\end{tabular}




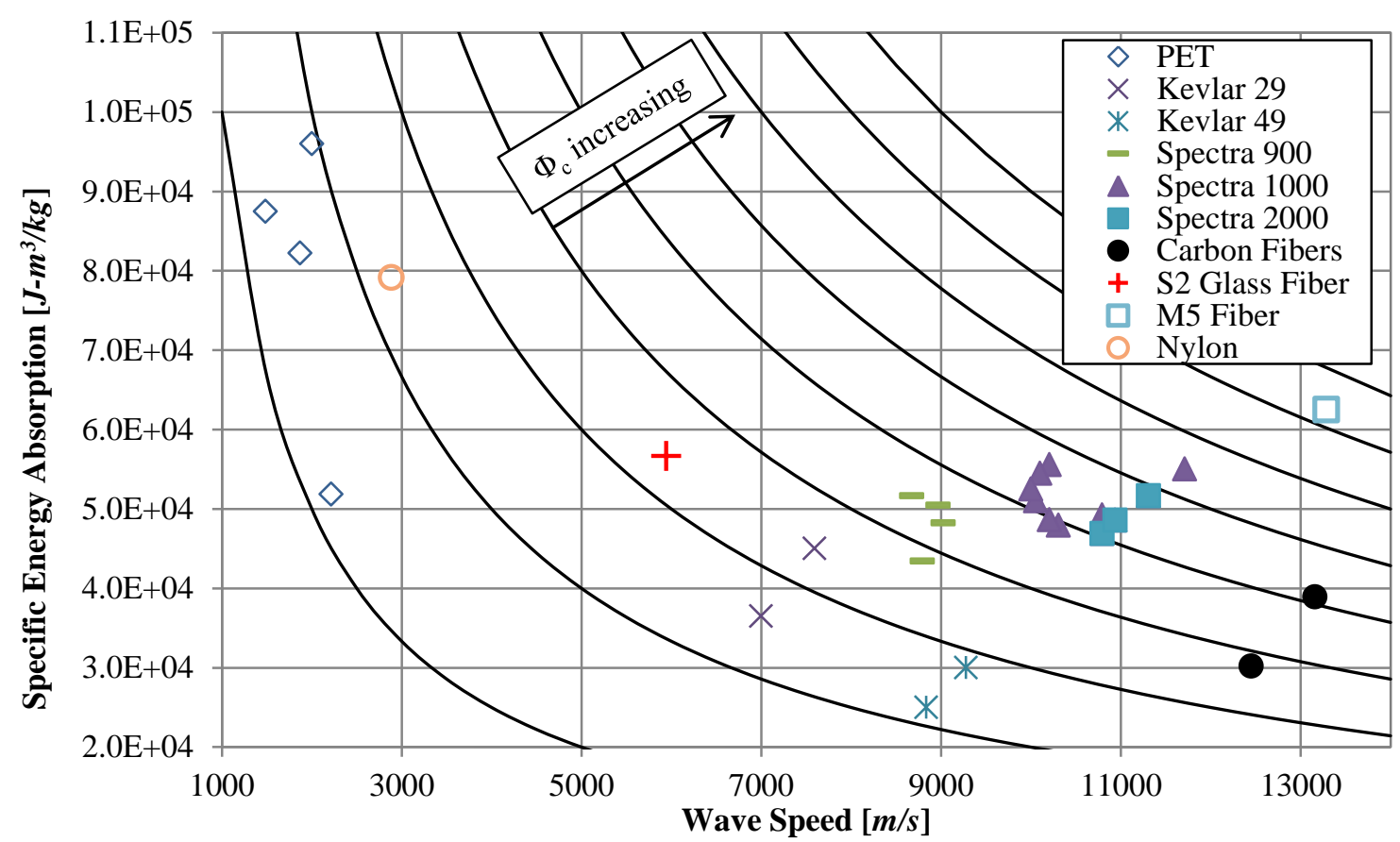

Figure 2.6 Fiber ballistic properties. Contours of constant $\Phi_{c}$ shown [33] to [38].

In order to study realistic ballistic fabrics, Roylance uses a grid model where secondary fibers are forced to move in the direction of stress waves traveling in the primary fibers, but are allowed some degree of slip. It was determined in this analysis that partial wave reflection occurs at crossover points, both impeding the transmission of the primary wave, and increasing the strain of the fiber at the crossover point. These two effects lead to premature failure of the fibers under ballistic loading [32], and give a good explanation as to why unidirectional fabrics are able to provide higher ballistic resistance compared to their woven counterparts [39].

\section{$2.5 \quad$ Ballistic Felts}

Ballistic composites include a category of non-woven textiles that, rather than using a secondary matrix material, rely on interfiber junctions to hold the textile together. These junctions are formed in a variety of ways including thermal calendaring and mechanical entanglement. Ballistic felts, formed by needle-punching entangled fibers, are studied in [1], [3], [40] to [43]. Felts have excellent ballistic properties at low areal densities and provide superior protection versus low velocity fragment projectiles. Chocron determined that the ballistic felt Dyneema ${ }^{\circledR}$ Fraglight $^{\circledR}$, at 
an areal density of only $0.6 \mathrm{~kg} / \mathrm{m}^{2}$, is capable of withstanding a $460 \mathrm{~m} / \mathrm{s}$ normal impact of a 1.1 gram $5.56 \mathrm{~mm}$ Fragment Simulating Projectile (FSP) [3]. However, ballistic felts do suffer from a number of deficiencies, namely, their range of applicability is limited to low velocity threats, and they require considerable deformation distances in order to stop a projectile [40].

The force-displacement behavior of a felt is highly nonlinear and involves significant evolution of the texture of the fabric arising from the rotation, realignment, and slippage of fibers. Felts are thought to stop projectiles not via efficient transport of stress waves or material inelastic deformation, but rather through a lossy, frictional, stick-slip mechanism. This so called stick slip mechanism, in which two interacting fibers alternate between being rigidly in contact and sliding upon one another, gives rise to macroscopic inelastic deformation, though the fibers themselves may only deform elastically. This mechanism is highly sensitive to fiber surface properties and the degree of confinement of a given fiber within the felt. A thicker felt with the same areal density as a thin felt will have a higher ballistic limit. This is thought to be an effect of the fiber mobility, with high compaction preventing fiber slippage from occurring [40].

Stress waves travel at a much lower velocity in felts than in unidirectional or woven composites, due to the low transmissibility of junctions and the tortuous path that an individual stress wave must traverse microscopically to accomplish significant displacement macroscopically. Because fibers are weakly held in place at junction points, rather than high magnitude stresses and wave reflection at these junction points, fibers instead slip and accelerate to the speed of the impacting projectile. Ipson experimentally determined the stress wave transmission in felts under ballistic impact by tracking material points on the felt using a series of spark gaps sewn into the back face of the felt [1]. The spark gaps were energized at known time increments leaving large bright spots on an exposed photographic plate which could be used to trace the motion of the felt. It was observed that the material initially displaces inward radially toward the impact site via longitudinal wave action, and then displaces transversely due to the macroscopic kink wave 
trailing the longitudinal wave. The macroscopic deformation described amounts to an axisymmetric extension of the single fiber analysis discussed earlier. Transverse velocity dropped off as it radiated outward from the impact site, indicating dispersion within the felt. The kinetic energy absorbed by a felt reaches a maximum at the felt's ballistic limit. This is a consequence of the drastic reduction in the volume of felt material involved in slowing the projectile as the ratio of projectile velocity to transverse wave velocity increases.

Ipson determined that the contact pressures between the projectile and felt were low, and never exceeded the elastic limit of the steel projectiles used in his experiments. Thus, the projectiles were essentially undeformed and stored a negligible amount of strain energy. A consequence of this observation is that the hardness and strength of the projectile matters very little during impact with a felt. This is both an advantage and disadvantage for the felt. It is an advantage versus high hardness armor piercing projectiles as the increased mechanical properties of the projectile affords no increase in penetration performance, but is a disadvantage in that a felt is unable utilize projectile deformation or erosion as a dissipation mechanism [44].

Ipson tested various felts composed of polypropylene, PET, Nylon, and Acrylic and determined that Nylon felts were the best performers with polypropylene registering a close second. Interestingly, Nylon had the lowest wave velocities of the fibers tested, but the highest strain to failure. This resulted in ballistic impacts having lower forces but higher contact times and displacements. Polypropylene, in contrast, possessed nearly the same ballistic limit, but achieved this through higher wave speeds and a higher failure stress rather than high strains to failure. PET felts had the lowest ballistic limit of the samples tested. A comparison of the ballistic limits for Nylon felt, 2024-T3 aluminum, and Hadfield steel versus a $5.56 \mathrm{~mm}$ FSP are shown in Table 2.3. 
Table 2.3 Areal density comparison of Nylon ballistic felt with metals of equivalent ballistic performance versus a $5.56 \mathrm{~mm}$ FSP [1].

\begin{tabular}{|c|c|c|c|}
\hline $\begin{array}{c}\text { Nylon Felt } A_{\mathrm{D}} \\
{\left[\mathrm{kg} / \mathrm{m}^{2}\right]}\end{array}$ & $\begin{array}{c}\text { Ballistic limit } \\
{[\mathrm{m} / \mathrm{s}]}\end{array}$ & $\begin{array}{c}\text { Al2024-T3 } \mathrm{A}_{\text {Deq }} \\
{\left[\mathrm{kg} / \mathrm{m}^{2}\right]}\end{array}$ & $\begin{array}{c}\text { Hadfield Steel } \mathrm{A}_{\text {Deq }} \\
{\left[\mathrm{kg} / \mathrm{m}^{2}\right]}\end{array}$ \\
\hline 0.64 & 229 & 7.80 & - \\
\hline 1.46 & 274 & 10.17 & 5.76 \\
\hline 1.80 & 305 & 11.53 & 6.10 \\
\hline
\end{tabular}

\subsection{Large Displacement Armor}

The concept of large displacement armor goes back to the impulse-momentum relation (Eq 2.2) where low forces but large deflections and relatively long time scales are used to arrest an incoming projectile. This is in contrast to classical plate armors which have very small deflections and generate high forces over short time periods. The aim of this work is to investigate thicksection nonwovens that undergo very large dynamic deflections, and to determine whether an armor that takes advantage of these large deflections can realize an improvement in ballistic performance. This amounts to sacrificing volumetric efficiency for the sake of increased protection, and is thus only suitable for applications in which an increase in the total thickness of the armor material can be tolerated.

The primary nonwoven studied in this thesis is polyester fiberfill manufactured by Polyester Fibers, LLC and sold under the brand name Mountain Mist ${ }^{\circledR}$ Fiberloft $^{\circledR}$. Fiberfill is utilized in stuffing of pillows and children's toys, but also sees use as an insulation material. It is comprised of crimped $30 \mu \mathrm{m}$ diameter polyethylene terephthalate staple fibers, with average crimped lengths of $\sim 25 \mathrm{~mm}$ and average fully extended lengths of $\sim 50 \mathrm{~mm}$. The fibers are mechanically entangled to form semi-consolidated batts that are then rolled and packaged. Because fiberfill is not needle punched, the fibers in it have a high degree of mobility. This makes handling of the fiberfill rather difficult as very low forces can alter its texture, and consequently its material properties. It is 
hypothesized that this increase in fiber mobility can lead to an increase in ballistic performance [42]. The selection of polyester fiberfill for this investigation was largely due to its wide availability and very low cost. PET fibers are not ideal for armor applications, but the format of the material should provide good insight into the mechanisms, and potential performance, of a non-needle punched, thick section, ballistic felt. 


\section{$3 \quad$ Preliminary Ballistic Testing}

\subsection{Experimental Procedure}

Early on in the experimental phase of this work, two sets of simple ballistic tests were undertaken to determine the usefulness of fiberfill in a ballistic armor application. In the first set of tests, fiberfill was stuffed into corrugated cardboard boxes and shot with two different types of .44 magnum ammunition. Projectiles were fired from a Winchester ${ }^{\circledR}$ Model 94.44 carbine at a range of $\sim 15 \mathrm{~m}$. Velocity measurements were not taken during the experiment, but the manufacturers' listed muzzle velocities are included in Table 3.1 below. The extended length of the barrel of the .44 carbine versus that of a .44 magnum handgun caused an increase in muzzle velocities of 140 $\mathrm{m} / \mathrm{s}$ in the case of Remington UMC ammunition (as determined in the second set of ballistic experiments), and is assumed to have caused a significant increase in the muzzle velocity of the MiWall ammunition as well. The kinetic energies of the projectiles upon impact with the sample were estimated to be $1 \mathrm{~kJ}$ and $2 \mathrm{~kJ}$ for the MiWall ammunition and the Remington ammunition respectively, when fired from the .44 carbine used in the experiment.

Table 3.1 Ammunition details (Manufacturer's Data)

\begin{tabular}{|c|c|c|c|c|}
\hline Ammunition Type & Mass $[g]$ & $\mathrm{V}[\mathrm{m} / \mathrm{s}]$ & $\begin{array}{c}\text { Kinetic Energy } \\
{[J]}\end{array}$ & Momentum $[\mathrm{kg}-\mathrm{m} / \mathrm{s}]$ \\
\hline $\begin{array}{c}\text { MiWall .44 Rem Mag } \\
\text { SP }\end{array}$ & 15.55 & 290 & 650 & 4.5 \\
\hline $\begin{array}{c}\text { Remington UMC .44 } \\
\text { Rem Mag JSP }\end{array}$ & 11.66 & 490 & 1400 & 5.7 \\
\hline
\end{tabular}

The second set of experiments tested the ballistic performance of varying densities of fiberfill compressed into $102 \mathrm{~mm}$ diameter $610 \mathrm{~mm}$ long cardboard tubes. The mass of the fiberfill was measured using a digital scale with the mass of the cardboard tube tared off. The same rifle firing only Remington UMC .44 magnum projectiles was used in these tests. Shots were fired at a 
distance of $3 \mathrm{~m}$ from muzzle to target. The average velocity of the Remington UMC ammunition was first determined, using an optical sensor chronograph, by firing shots over the test apparatus without a sample present. The average velocity of the five shots fired was $632 \mathrm{~m} / \mathrm{s}$, equating to a kinetic energy of $2332 \mathrm{~J}$. For the experiment, projectiles were fired through the armor samples, and the exit velocity of the projectile was measured for multiple shots per sample. The difference in the exit velocity with and without a sample present was used to calculate the kinetic energy absorbed by the armor. A schematic of the experimental setup is show in Figure 3.1.

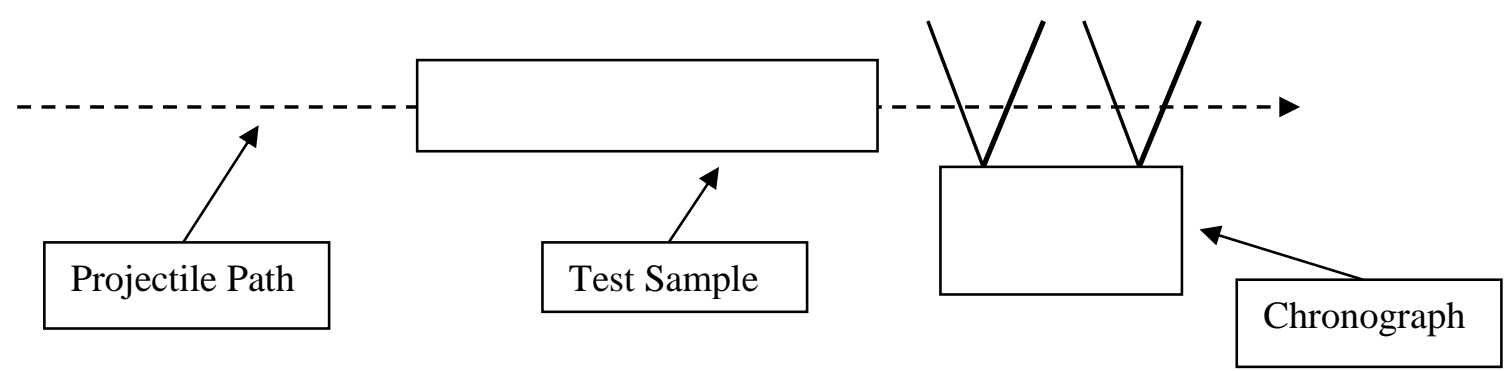

Figure 3.1 Schematic of the second ballistic experimental setup.

\subsection{Results}

In the first set of experiments all seven MiWall projectiles were defeated in 0.3 to 0.35 meters of compressed PET fiberfill. None of the Remington projectiles was defeated within the 0.6 meters total thickness of the fiberfill samples.

Defeated projectiles were extracted from the samples and the surrounding fibers were examined at both a macroscopic and microscopic level. On the leading edge of the projectiles, an envelope of compressed fibers formed cocoon-like structures that also entrained surrounding fibers (Figure 3.2 and Figure 3.3). The path of each defeated projectile was marked by a densified, highly aligned, and twisted tail (Figure 3.4 and Figure 3.5) surrounded by a less dense "hole" plowed by the projectile from the entrance to the point of defeat. A similar structure was left in the wake of the Remington ammunition, from the point of entry to the exit hole. Multi-hit performance was enhanced by the residual compressive stress brought about by packing the fibers into the box. 
This stress serves to backfill the "holes," formed by the projectiles that pass through the armor. The paths traced by the lower velocity projectiles, as evidenced by both tails and holes, indicated that projectiles became unstable and veered from their initial trajectories. The tortuous paths taken by destabilized projectiles enhanced the volume of armor material participating in ballistic defeat.

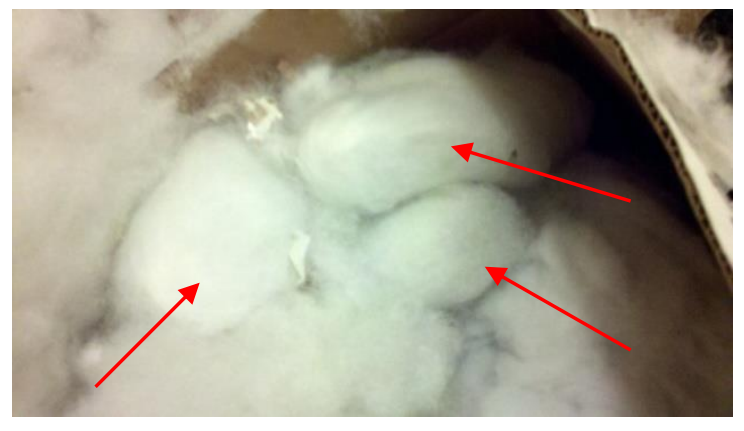

Figure 3.2 "Cocoon" structures surrounding defeated projectiles.

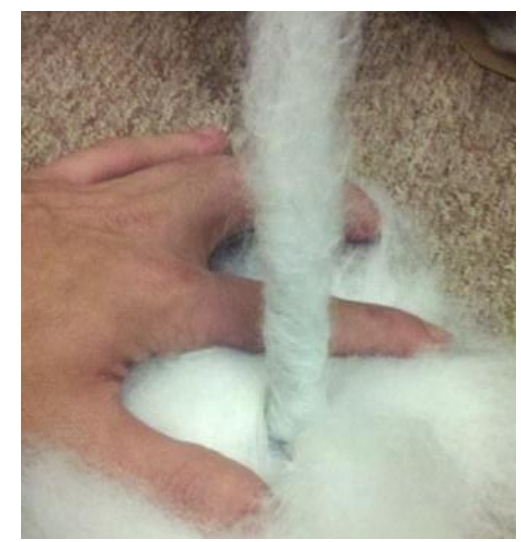

Figure 3.4 "Tail" structure following projectile path.

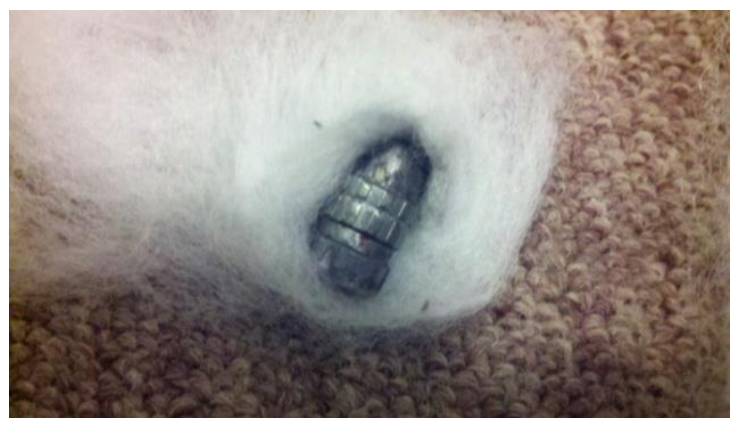

Figure 3.3 Defeated projectile being extracted from a "cocoon." The tail on this projectile has already been removed.

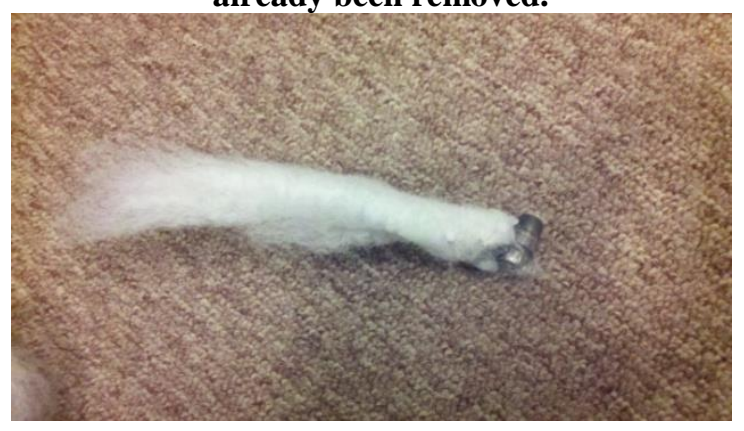

Figure 3.5 "Tail" emanating from the side of a defeated projectile.

A sample of fibers in the projectile affected area, where friction and melting appeared to occur at the macroscopic level, was extracted, using a razor blade, and examined under a scanning electron microscope (SEM) (Figure 3.6 and Figure 3.8). Projectile affected fibers were compared to fibers in the virgin state (Figure 3.7 and Figure 3.9). All samples were mounted to a pressure sensitive adhesive coated conductive platen prior to examination, but neither sample was sputter coated. Significant deformation, sliding friction, and melting were evident from the SEM images. 
In addition to melted and worn areas, fibers from the affected region showed significant flattening, and exhibited a greater degree of kinking than fibers from unaffected regions.

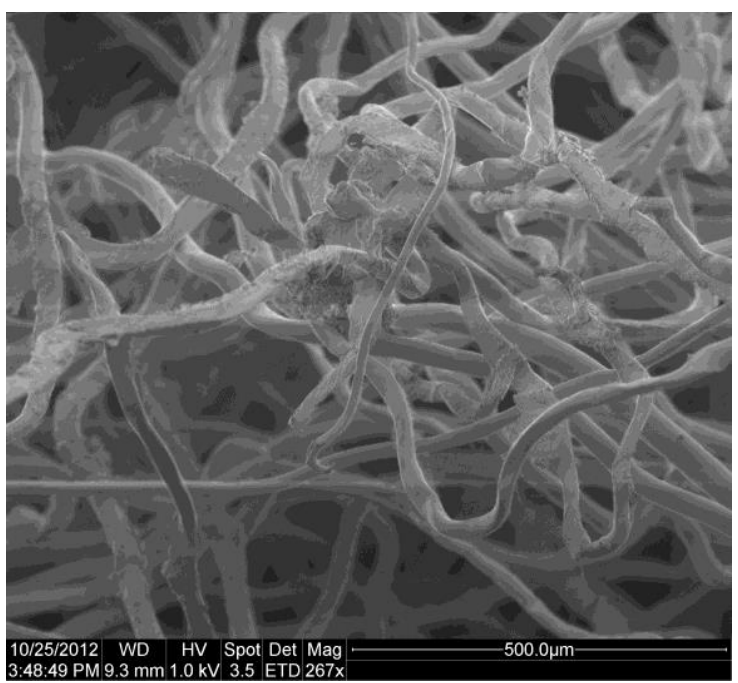

Figure 3.6 Fibers from the projectile affected region showing melting and adhesion.

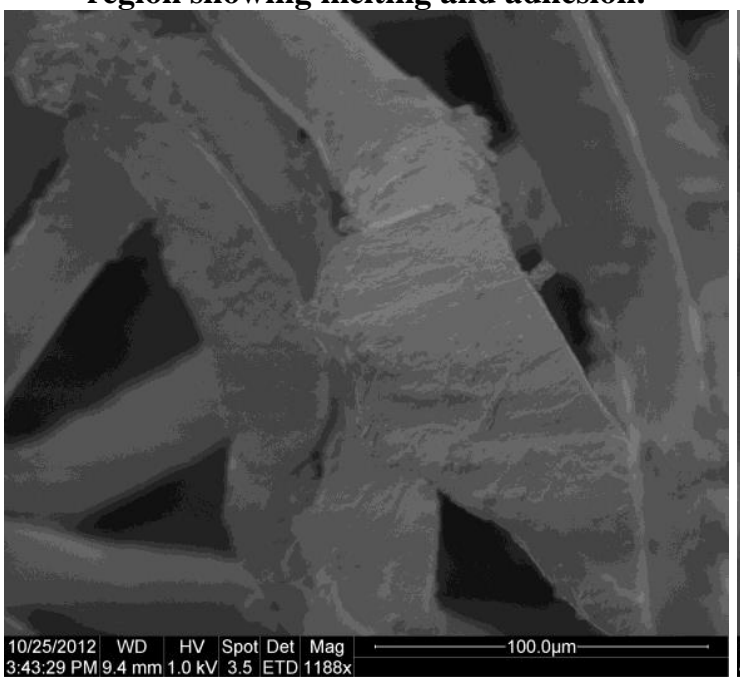

Figure 3.8 Close up of fiber from the affected region showing inter-fiber friction wear and plastic deformation.

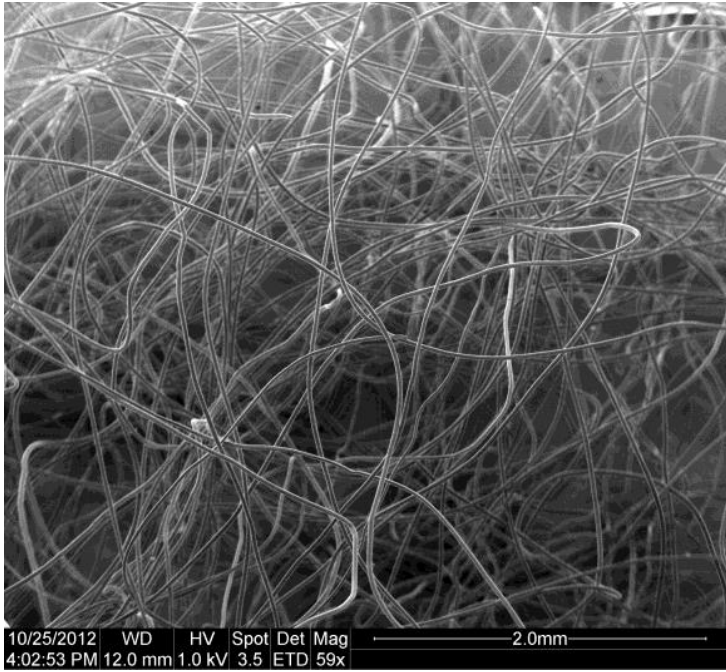

Figure 3.7 Fibers from a region unaffected by projectiles.

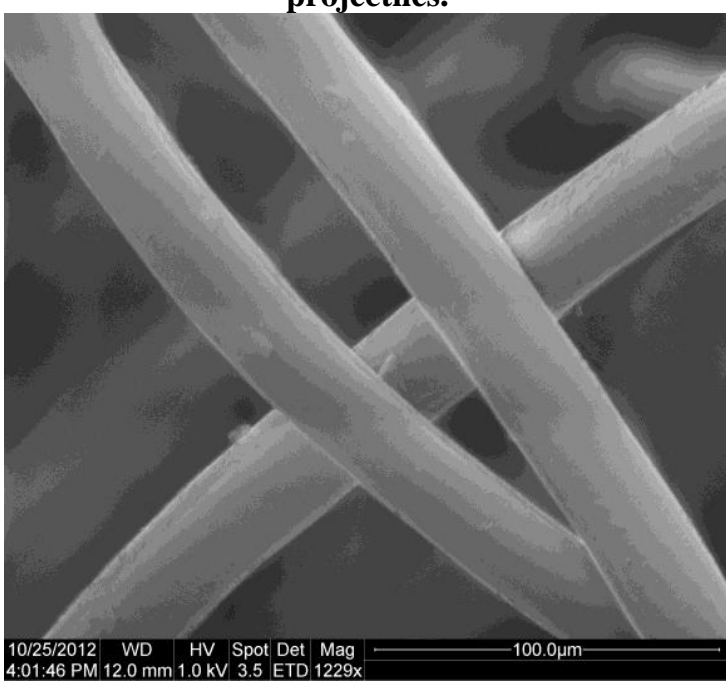

Figure 3.9 Pristine fibers from unaffected region.

The results of the second experiment are summarized in Figure 3.10. Interestingly, even after multiple shots were fired in essentially the same location, very little difference in the absorbed kinetic energy was observed. For the $46.2 \mathrm{~kg} / \mathrm{m}^{2}$ sample, the energy absorbed for the first, second, and third shot respectively was $949 \mathrm{~J}, 1135 \mathrm{~J}$, and $993 \mathrm{~J}$. 
Paths taken by projectiles in the second set of ballistic experiments were noted to display none of the tortuosity seen in the initial experiment. This is thought to be due to a combination of effects, namely, an increase in the projectile velocities, making the projectile less prone to destabilization due to an unbalance of forces, and a near-wall condition that may serve to direct the projectile along the axis of the constraint tube.

The results from these early ballistic experiments served to inform the remainder of the experimental studies conducted on fiberfill based armors. It was clear that controlled ballistic tests, involving a variation of velocity and "far-field" boundary conditions, were necessary to accurately determine the ballistic limit of fiberfill armor. Furthermore, a study of the mechanisms of fiber realignment and interfiber sliding under different loading conditions would be important to gaining insight into fiberfill's potential as an armor material.

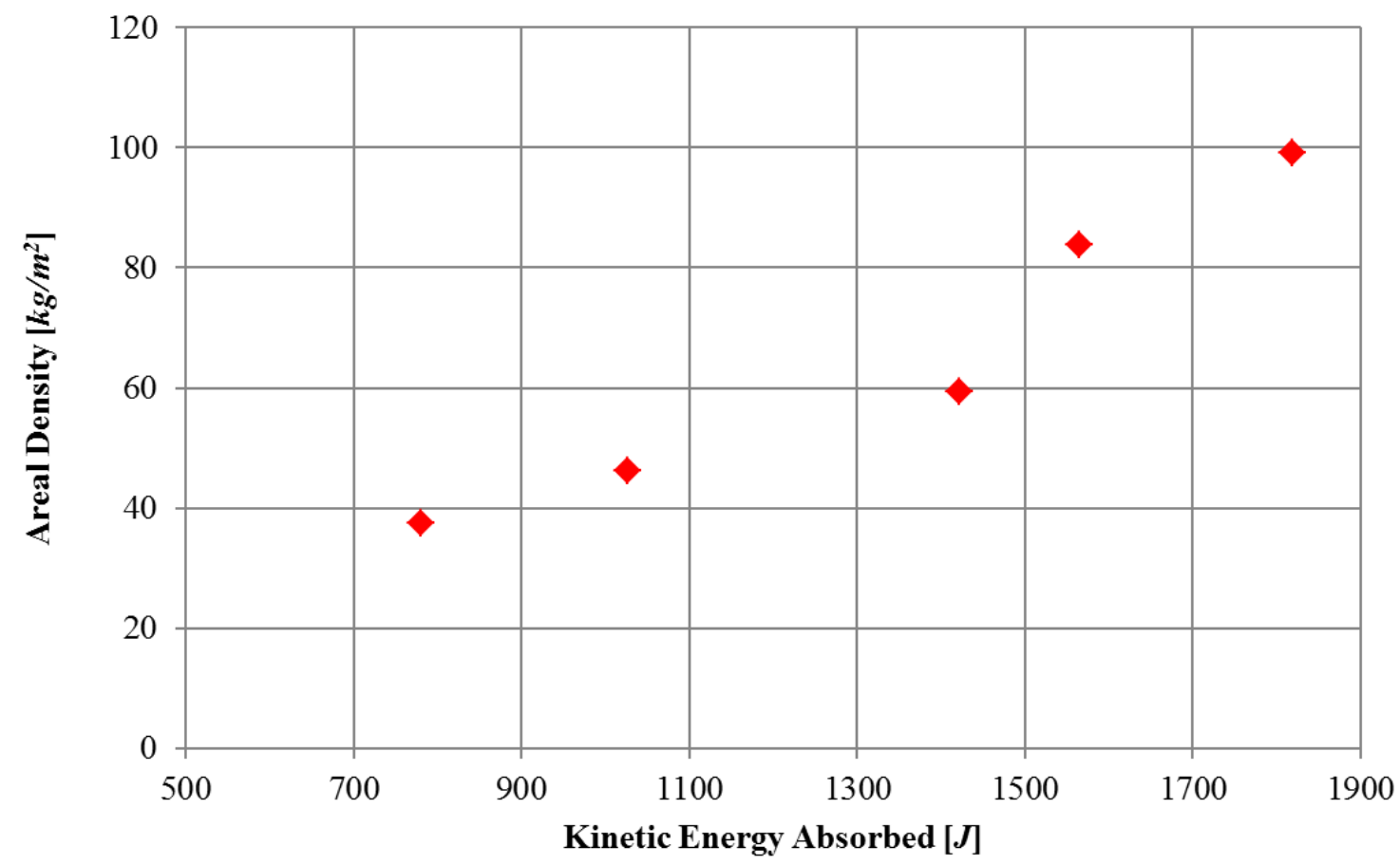

Figure 3.10 Results of the exit velocity experiments, values shown are averages of multiple shots fired into the same sample. 


\section{Mechanical Testing and Micromechanics}

Fiberfill was mechanically characterized in the deformation modes thought to be relevant to ballistic impact. While the loading conditions in ballistic impact are both mixed and occurring at various strain rates, isolating those loading conditions to homogenous, constant strain rate cases should give valuable insight into fiberfill's mechanical behavior. Where possible, elevated strain rates were studied, but, due to practical limitations, the majority of the material characterization tests were performed in a quasistatic manner. The response of fiberfill to homogenous deformations of uniaxial compression, uniaxial tension, and shear were investigated. For each deformation mode, a short review section and discussion on the underlying micromechanics precedes the experimental discussion. Where applicable, theoretical predictions of the micromechanical theory are compared with experimental results.

\subsection{Compression}

\subsubsection{Micromechanics of compression}

The compression behavior of entangled fiberwebs was studied by van-Wyk [45] who was investigating the compression behavior of wool. He concluded that the governing mechanism was fiber bending between contact points. Utilizing elementary beam theory, van-Wyk determined that the compression pressure should vary according to:

$$
\sigma=\mathrm{kE} \Phi^{\mathrm{n}}
$$

Where $\mathrm{k}$ is a fit parameter that accounts for geometric effects, $\mathrm{n}$ accounts for fiber distribution, $\mathrm{E}$ is the fiber elastic modulus, and $\Phi$ is the volume fraction of fibers.

This equation can also be expressed in terms of the true strain, $\epsilon=\ln \left(\frac{\mathrm{x}}{\mathrm{x}_{0}}\right)$, rather than the volume fraction [46]: 


$$
\sigma=\mathrm{Ae}^{\mathrm{b} \epsilon}
$$

here $\mathrm{b}$ is the same as the exponent $\mathrm{n}$ in the Toll van-Wyk equation, and $\mathrm{A}$ is a lumped modulus parameter with units of pressure.

Toll later extended van-Wyk's theory to account for both planar (2 dimensional) and bulk (3 dimensional) fiber orientation distribution, and accounted for the maximum unforced packing of fibers [47]. This modification is expressed as:

$$
\sigma=\mathrm{kE}\left(\Phi^{\mathrm{n}}-\Phi_{0}^{\mathrm{n}}\right)
$$

At sufficiently high values of $\Phi, \Phi_{0}$ can be neglected. Toll's analysis predicts that the contact in planar fibers can no longer be approximated as point contact, and approaches line contact as the planar fibers are increasingly aligned. This affects the exponent, n, in van-Wyk's equation, with random three dimensional configurations taking on the exponent $\mathrm{n}=3$, and planar splays taking on the exponent of $n=5$. Increasing alignment increases the exponent depending on the orientation distribution and the degree of fiber-fiber slip [48] to [50]. Hysteresis under compressive loading is discussed in Carnaby [51]. Experimental studies of entangled fiber compression can be found in Mezeix [46], and numerical investigation of van-Wyk's theory can be found in [52] to [54].

\subsubsection{Compression experimental setup and procedure}

Compressions tests were performed for strain rates (defined as $\dot{\epsilon}=\frac{\mathrm{V}}{\mathrm{L}_{0}}\left[\frac{1}{s}\right]$ where $\mathrm{V}$ is the velocity of the cross head and $\mathrm{L}_{0}$ is the initial height of the sample) ranging from $2.65 \times 10^{-4}$ to $1.061\left[\frac{1}{s}\right]$. All compression tests were performed on a servo-hydraulic Instron ${ }^{\circledR}$ universal test machine. The compression test fixture utilized a transparent constraint tube to keep the fiberfill sample in place between the two, $197 \mathrm{~mm}$ diameter platens (Figure 4.1). This constraint tube does not allow unconstrained radial expansion of the samples under test, and thus the test is not truly uniaxial. However, since fiberfill is highly compressible, the effect of the constraint tube's presence is 
assumed to be negligible. The effect of friction at the walls of the constraint tube was also neglected.

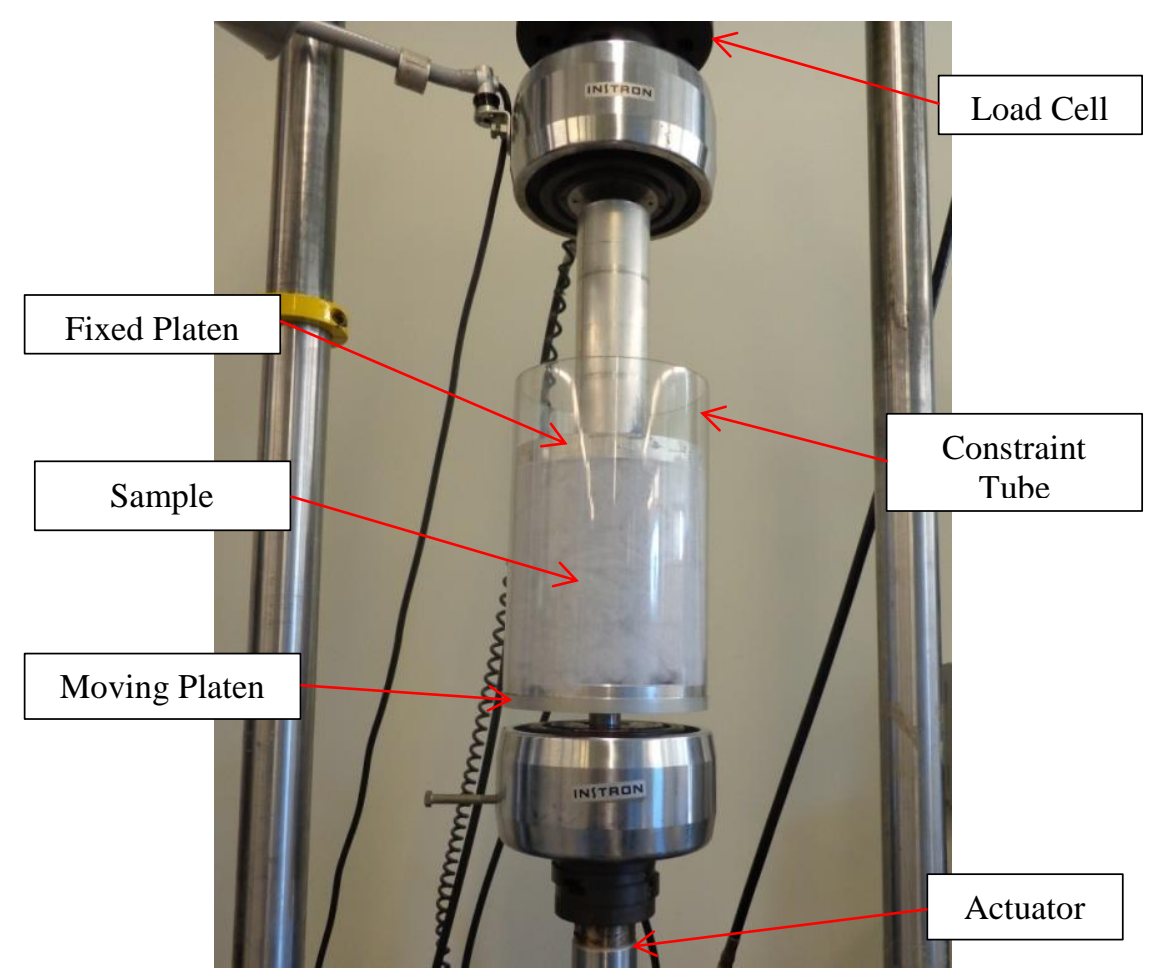

Figure 4.1 Compression test fixture mounted to servo hydraulic test frame

Compression samples were prepared by removing portions of fiberfill from the master roll, and placing them in the constraint tube, layer-by-layer until the desired sample mass was achieved. Material was added or removed from each layer as necessary for that individual layer to correctly conform to the inside of the constraint tube. This layering method imparted preferred alignment to the individual layers in the direction transverse to the loading axis, and thus altered the structure of the fiberfill. Ideally, randomly entangled preforms would be fabricated inside of the constraint tube, but attempts to do this using blown air were unsuccessful.

The procedure for conducting the individual compression tests was as follows:

- Balance the load cell and actuator readings

- Begin recording data 
- Lower the crosshead to a fixed displacement defined by the yellow shaft collar in Figure 4.1, thus pre-compressing the sample

- Lock the crosshead in place

- Start the actuator, which moves upward at a fixed velocity throughout the test

- End the test once a maximum load of $35 \mathrm{kN}$ is reached

Due to the tests not starting in a zero stress state, the results had to be "toe-corrected." To perform the toe correction a linear fit of the data in the low force regime was performed and the displacement necessary to reach an unstressed state was determined from the slope and intercept of this fit. The gage length and strain are then adjusted accordingly to achieve an unstressed state at zero strain (Figure 4.2).

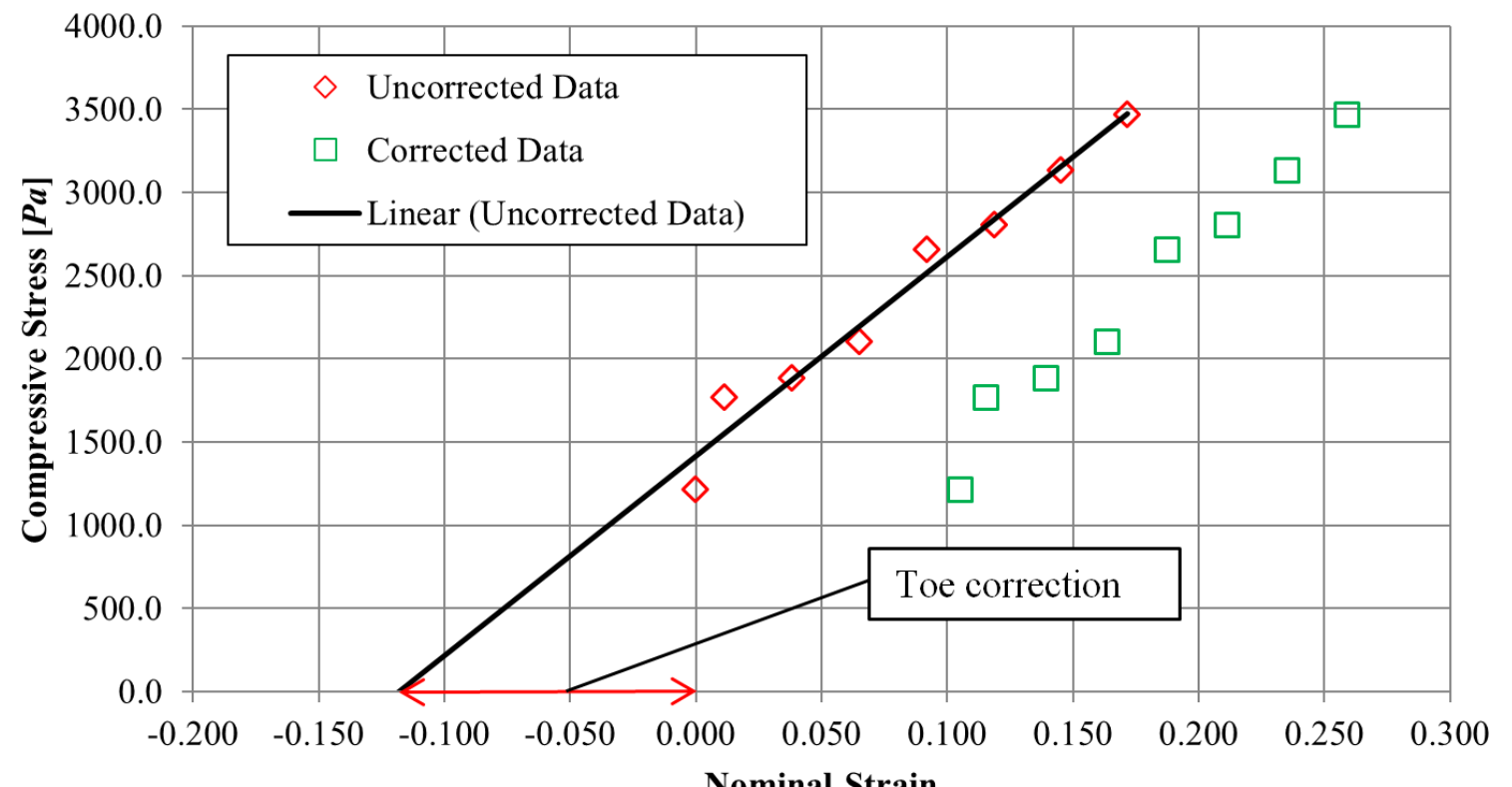

Figure 4.2 Example of toe correction procedure for compression data

Relaxation tests were performed in a fashion similar to the compression tests, but rather than stopping at a defined force, the actuator was set to stop at a defined displacement. The actuator 
rapidly ramped to the desired displacement at which point it ceased moving. The reduction in the stress over time at this displacement was then recorded until it appeared to reach steady state. This "relaxation," is due to fiber reorientation and the escape of air from the fiberfill.

\subsubsection{Compression Results}

The results of all of the compression tests are shown in Figure 4.3 and Figure 4.4. A nonlinear least squares curve fit was performed on each individual data set and the average of these parameters was used to provide the master curve fit, shown in black. Table 4.1 summarizes the results of the curve fitting. $\mathrm{kE}, \Phi_{0}$, and $\mathrm{n}$ refer to the parameters in the Toll van-Wyk equation, and $\mathrm{A}$ and $\mathrm{b}$ refer to the parameters in the Meziex version of the Toll van-Wyk equation [46].

The data is in excellent agreement with the power law prediction, but did show significant dispersion, especially in the low compaction range. This is likely due to the variability in samples due to non-ideal sample preparation procedures. Nonetheless, an exponent of 3.6 was obtained indicating that the samples were not three dimensionally isotropic in their randomness, but that they were not excessively aligned to the point where they behaved more like a two dimensional fiber splay.

No rate dependence was evident in the range of strain rates tested. This was surprising due to the rate dependent nature of PET as well as the expected poroelastic contribution to the stress arising from the air contained in each sample [55]. However, while four decades of strain rates were sampled, the absolute value of these rates was likely too low to begin seeing evidence of these effects.

Toll made a generalized prediction for the maximum unforced packing of arbitrarily oriented particles. For three dimensional, randomly oriented, nondisperse fibers Toll's prediction for $\Phi_{0}$ is:

$$
\Phi_{0}=\frac{4}{3+\overline{\mathrm{r}}}
$$


Where $\overline{\mathrm{r}}=\frac{1.7 \times 10^{-3}}{30 \times 10^{-6}}\left[\frac{\mathrm{m}}{\mathrm{m}}\right]$ is the fiber aspect ratio, taken as the average length between crimps divided by the average fiber diameter. The resulting prediction for the maximum unforced packing is $\Phi_{0}=6.7 \times 10^{-2}$. This prediction is somewhat higher than the average result obtained in the compression experiments (Table 4.1). Likely sources of error include experimental inaccuracy in the low force-displacement regime and non-ideal packing, which leads to dispersity and nonrandom alignment of fibers.

Table 4.1 Strain rates and curve fit parameters for compressions samples

\begin{tabular}{|c|c|c|c|c|c|c|}
\hline Sample & $\dot{\boldsymbol{\epsilon}}[\mathbf{1} / \mathbf{s}]$ & $\mathbf{k E}[\boldsymbol{P a}]$ & $\mathbf{n}$ & $\boldsymbol{\Phi}_{\mathbf{0}}$ & $\mathbf{A}[\boldsymbol{P a}]$ & $\mathbf{b}$ \\
\hline P110 & $2.65 \mathrm{E}-01$ & $1.40 \mathrm{E}+07$ & 3.51 & $6.07 \mathrm{E}-07$ & 243.6 & 3.51 \\
\hline P111 & $2.65 \mathrm{E}-01$ & $2.07 \mathrm{E}+07$ & 3.74 & $1.19 \mathrm{E}-06$ & 214.1 & 3.72 \\
\hline P112 & $2.65 \mathrm{E}-04$ & $1.30 \mathrm{E}+07$ & 3.49 & $6.31 \mathrm{E}-07$ & 242.4 & 3.49 \\
\hline P113 & $2.65 \mathrm{E}-04$ & $1.97 \mathrm{E}+07$ & 3.66 & $3.06 \mathrm{E}-02$ & 220.8 & 3.66 \\
\hline P114 & $2.65 \mathrm{E}-04$ & $1.72 \mathrm{E}+07$ & 3.60 & $2.38 \mathrm{E}-07$ & 223.2 & 3.60 \\
\hline P115 & $2.65 \mathrm{E}-03$ & $1.72 \mathrm{E}+07$ & 3.60 & $3.15 \mathrm{E}-07$ & 223.2 & 3.60 \\
\hline P116 & $2.65 \mathrm{E}-03$ & $2.06 \mathrm{E}+07$ & 3.72 & $4.65 \mathrm{E}-02$ & 202.7 & 3.73 \\
\hline P117 & $2.65 \mathrm{E}-03$ & $1.54 \mathrm{E}+07$ & 3.50 & $2.66 \mathrm{E}-06$ & 251.6 & 3.50 \\
\hline P118 & $2.65 \mathrm{E}-03$ & $2.06 \mathrm{E}+07$ & 3.68 & $4.65 \mathrm{E}-02$ & 222.6 & 3.71 \\
\hline P119 & $2.65 \mathrm{E}-03$ & $2.68 \mathrm{E}+07$ & 3.87 & $5.68 \mathrm{E}-02$ & 193.3 & 3.90 \\
\hline P120 & $2.65 \mathrm{E}-02$ & $1.97 \mathrm{E}+07$ & 3.61 & $2.47 \mathrm{E}-06$ & 240.0 & 3.61 \\
\hline P121 & $2.65 \mathrm{E}-02$ & $2.30 \mathrm{E}+07$ & 3.70 & $4.34 \mathrm{E}-02$ & 231.2 & 3.72 \\
\hline P122 & $2.65 \mathrm{E}-02$ & $2.19 \mathrm{E}+07$ & 3.72 & $5.60 \mathrm{E}-02$ & 229.0 & 3.75 \\
\hline P123 & $2.65 \mathrm{E}-02$ & $1.84 \mathrm{E}+07$ & 3.57 & $3.35 \mathrm{E}-06$ & 248.6 & 3.57 \\
\hline P124 & $2.65 \mathrm{E}-02$ & $2.40 \mathrm{E}+07$ & 3.73 & $4.81 \mathrm{E}-02$ & 230.5 & 3.75 \\
\hline P125 & 1.06 & $1.35 \mathrm{E}+07$ & 3.42 & $1.93 \mathrm{E}-02$ & 338.3 & 3.42 \\
\hline P126 & 1.06 & $1.95 \mathrm{E}+07$ & 3.57 & $5.03 \mathrm{E}-02$ & 304.3 & 3.59 \\
\hline P127 & 1.06 & $1.74 \mathrm{E}+07$ & 3.52 & $2.80 \mathrm{E}-02$ & 228.6 & 3.52 \\
\hline P128 & 1.06 & $1.78 \mathrm{E}+07$ & 3.53 & $5.54 \mathrm{E}-02$ & 355.7 & 3.56 \\
\hline P129 & 1.06 & $1.35 \mathrm{E}+07$ & 3.40 & $4.12 \mathrm{E}-02$ & 410.9 & 3.41 \\
\hline P130 & 1.06 & $1.54 \mathrm{E}+07$ & 3.46 & $5.69 \mathrm{E}-02$ & 394.7 & 3.49 \\
\hline Average & - & $1.85 \mathrm{E}+07$ & 3.60 & $2.76 \mathrm{E}-02$ & 259.5 & 3.61 \\
\hline Standard Deviation & - & $3.69 \mathrm{E}+06$ & 0.12 & $2.40 \mathrm{E}-02$ & 62.6 & 0.13 \\
\hline & & & & & & \\
\hline
\end{tabular}




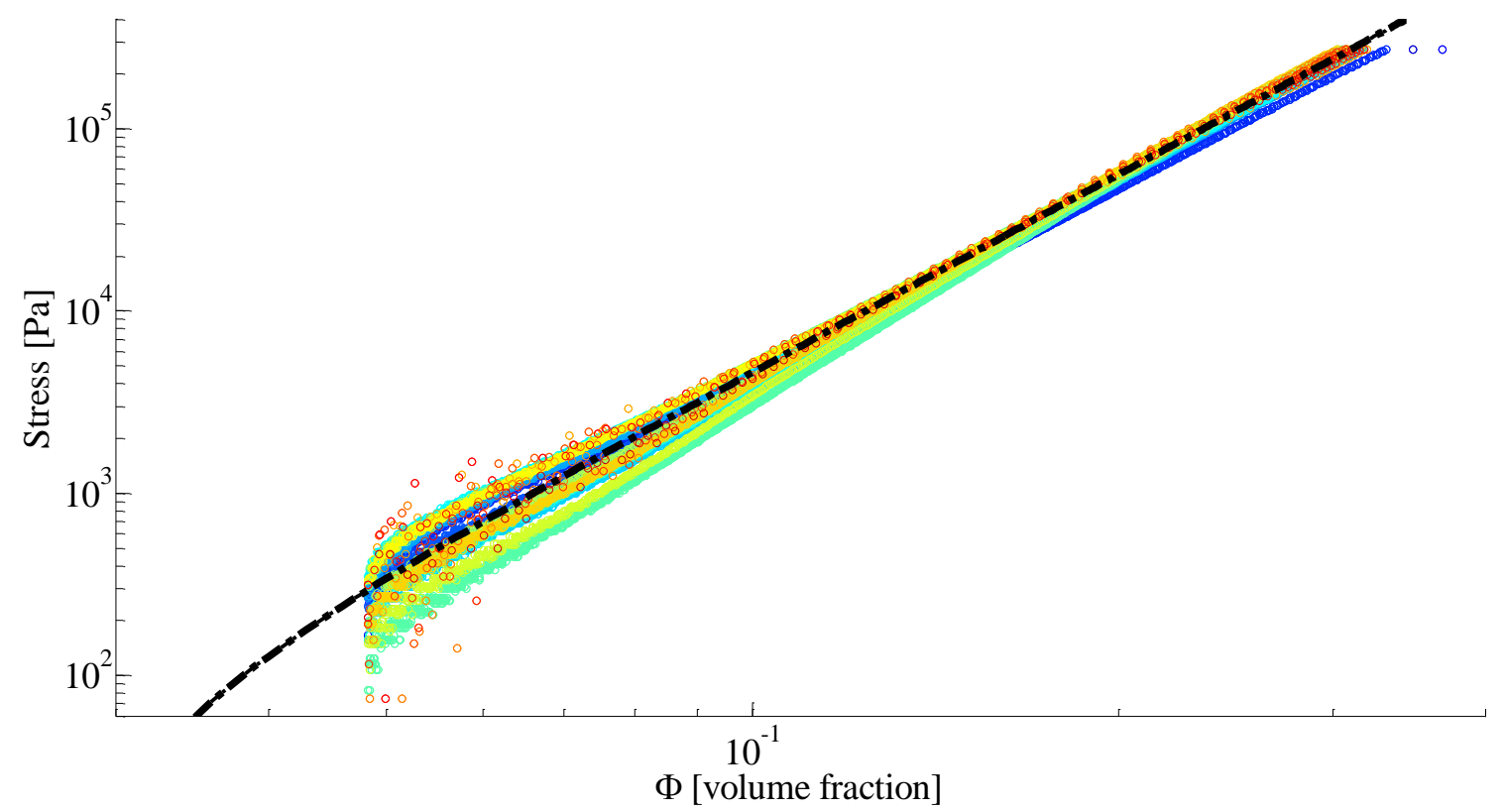

Figure 4.3 Compressive stress versus volume fraction (all data shown). Fit to the Toll van-Wyk equation shown in black.

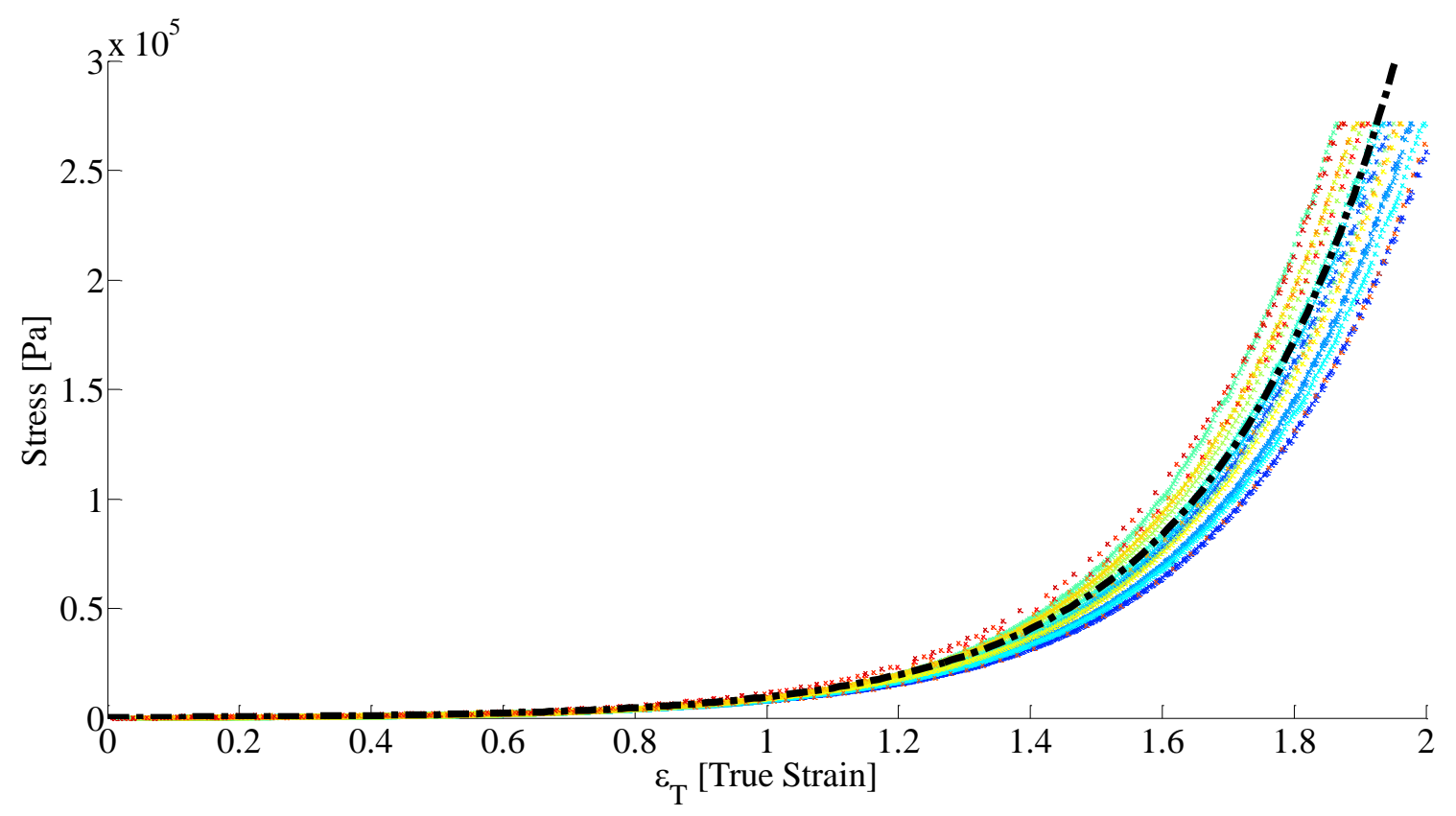

Figure 4.4 Compressive stress versus true strain.

Fit to the Meziex form of the Toll van-Wyk equation shown in black.

Representative relaxation results are shown in Figure 4.5. The data are fit to the standard linear solid viscoelastic model, which consists of a spring in parallel with a Maxwell element as shown 
in Figure 4.6. The solution to the ordinary differential equation that describes the response of this model to a step input of strain is (see for example: Mase [56]):

$$
\mathrm{E}=\mathrm{E}_{\mathrm{f}} \exp (-\beta \mathrm{t})+\mathrm{E}_{\mathrm{c}}
$$

Where $\beta=\frac{E_{D}}{\eta}$. The parameters determined by the curve fit are shown in Table 4.2 below. These parameters are later used in modeling drop test experiments.

Table 4.2 Viscoelastic relaxation fit parameters

\begin{tabular}{|c|c|}
\hline $\mathrm{E}_{\mathrm{D}}[\mathrm{Pa}]$ & $2.24 \mathrm{E}+04$ \\
\hline$\beta[1 / s]$ & 1.649 \\
\hline $\mathrm{E}_{\mathrm{c}}[\mathrm{Pa}]$ & $3.65 \mathrm{E}+04$ \\
\hline
\end{tabular}

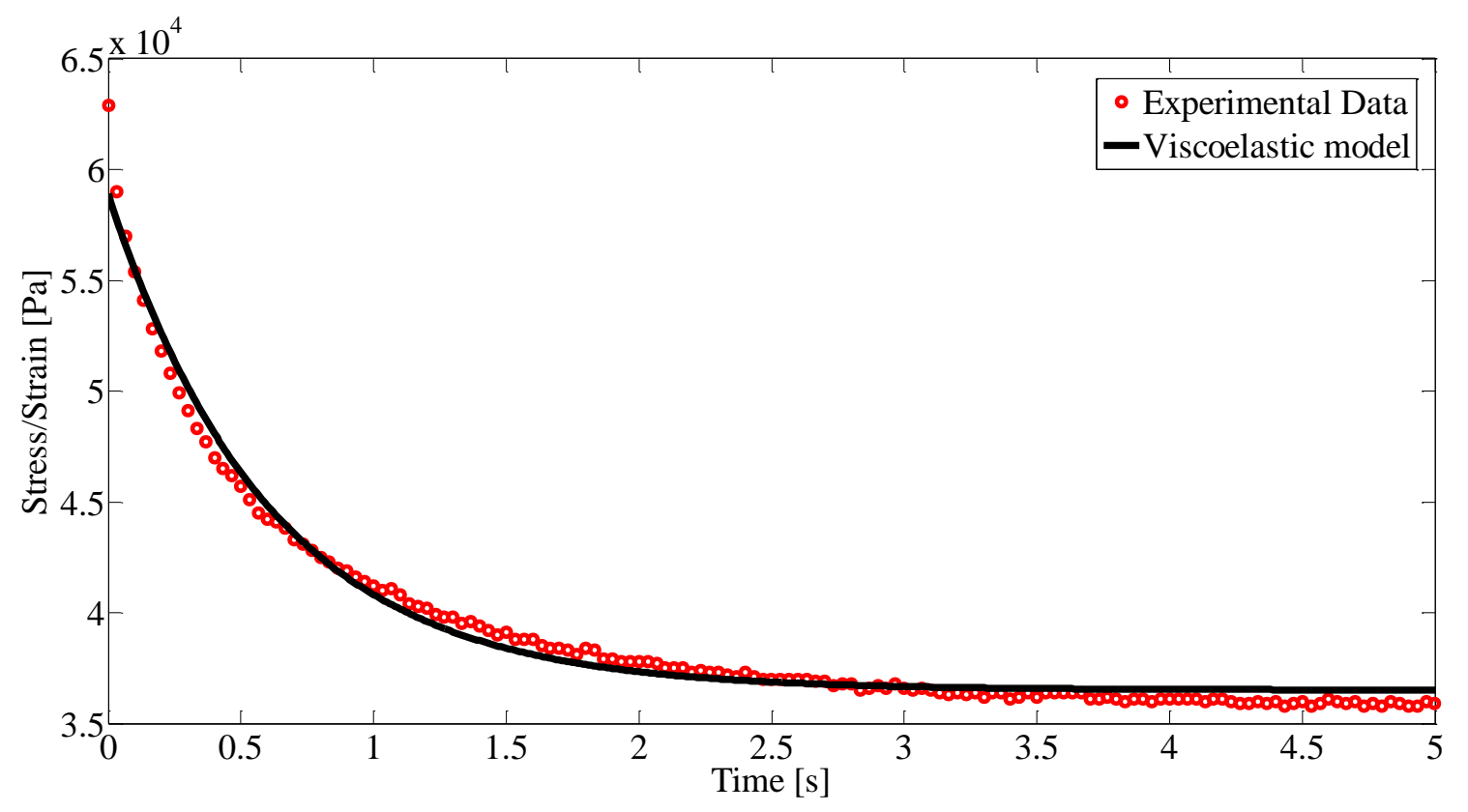

Figure 4.5 Relaxation data and curve fit 


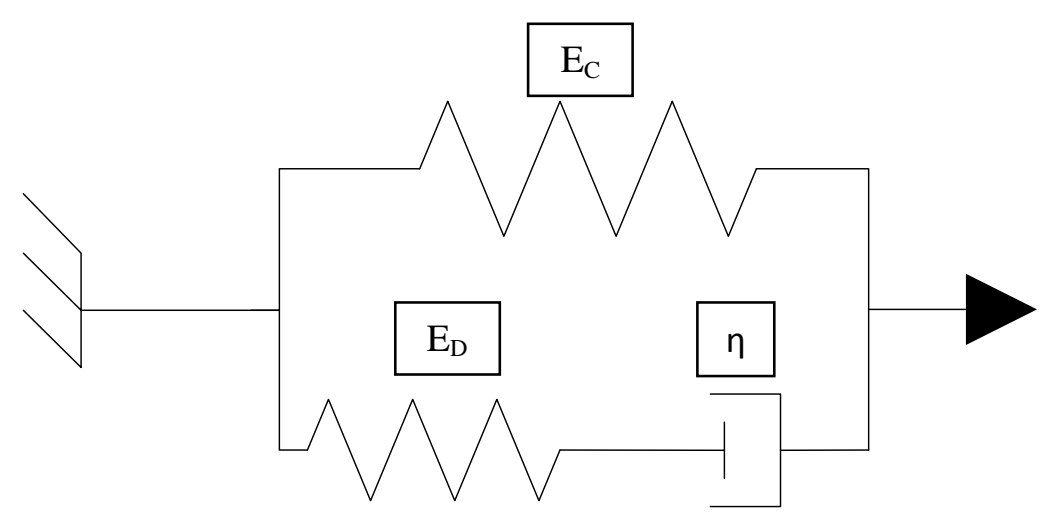

Figure 4.6 Standard linear solid viscoelastic model

\subsection{Drop Tests}

\subsubsection{Micromechanics of drop test experiments}

The drop test experiments conducted in this work were designed to closely mimic the compression tests performed in the previous section. The micromechanics for these high-rate compression tests are expected to be nearly identical to those seen in the quasistatic case, with the exception that rate effects should be visible as strain rates are increased above a certain threshold. The strain rate hardening in high rate compression tests is expected to arise from two sources:

1. Interfiber friction opposing the rapid reorientation of fibers during compression

2. Viscous effects caused by air trying to escape from the fiberfill/test fixture

As the strain rates grow very high, for low-density fiberfill, the contribution from entrapped air is expected to dominate the compression response [55]. This is very similar to the behavior of open celled foams under high strain rate compressive loading.

\subsubsection{Drop test experimental setup and procedure}

In order to determine the effect of high strain rates on the compression response of fiberfill a series of drop tests were performed. A test fixture similar to the one used for compression tests was used in these experiments (Figure 4.7). In the experiments, the fixture is mounted in a 
Dynatup drop tower, and a weighted crosshead, with a mass of $6.15 \mathrm{~kg}$, is dropped on samples from varying heights. The force on the crosshead is determined from a conservation of momentum analysis where the acceleration of the cross head is calculated by twice differentiating displacement data with respect to time and multiplying the result by the mass of the crosshead. Displacement versus time data is collected using a Phantom high-speed camera (Figure 4.8) recording at a rate of 15,000 frames per second. The data is processed using a Matlab script that tracks the motion of a black dot drawn on the crosshead and smoothes the displacement data using a multi-point averaging scheme. The force acting on the cross head is taken to be equal to and opposite the force acting on the fiberfill sample under test. The stress in the fiberfill is:

$$
\sigma=\frac{m_{c}}{A_{s}}\left(\frac{d^{2} x}{{d t^{2}}^{2}}\right)
$$

Where $m_{c}$ is the mass of the crosshead, $A_{s}$ is the area of the fiberfill sample, $x$ is the displacement of the crosshead, and $t$ is time. The body force due to gravity is ignored, because of its relatively low magnitude. The double differentiation is very sensitive to any noise or error in the displacement data, hence the use of multi-point averaging to reduce these effects.

Four different sets of test parameters were used, as described in Table 4.3. Two different densities were studied at two different drop heights, and each test ID \# was conducted a minimum of three times.

Table 4.3 Parameters for drop test experiments

\begin{tabular}{|c|c|c|c|}
\hline TestID & Drop height $[\mathrm{m}]$ & Impact velocity $[\mathrm{m} / \mathrm{s}]$ & Density of fiberfill $\left[\mathrm{kg} / \mathrm{m}^{3}\right]$ \\
\hline 1 & 0.77 & 3.91 & 66.7 \\
\hline 2 & 0.77 & 3.91 & 44.4 \\
\hline 3 & 0.35 & 2.62 & 66.7 \\
\hline 4 & 0.35 & 2.62 & 44.4 \\
\hline
\end{tabular}




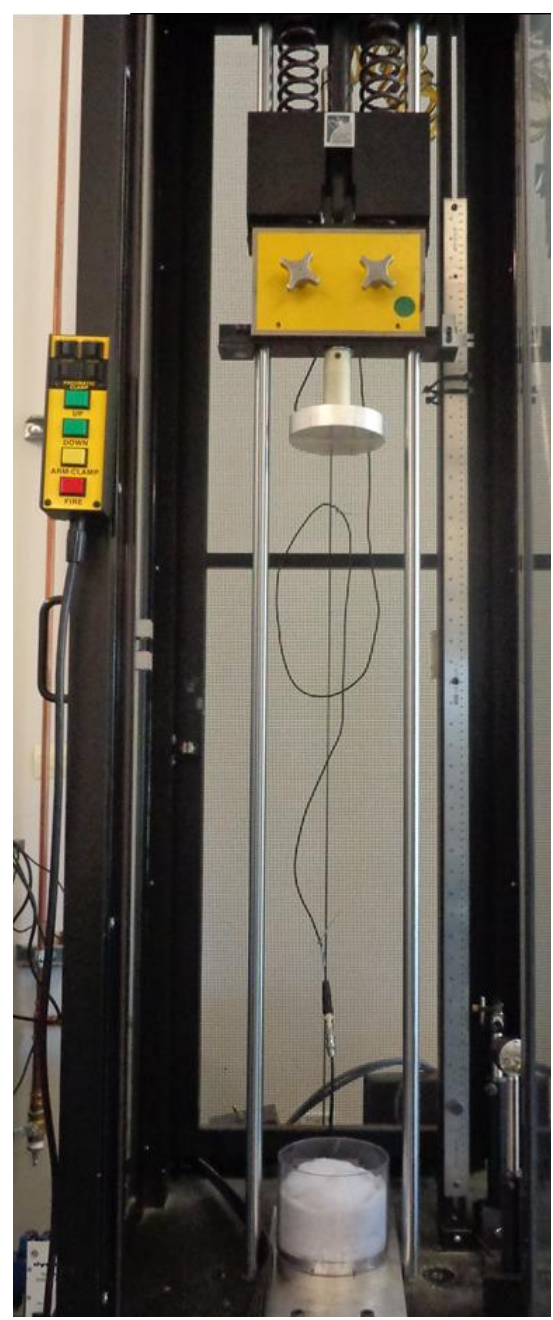

Figure 4.7 Drop test fixture mounted in the Dynatup drop tower.

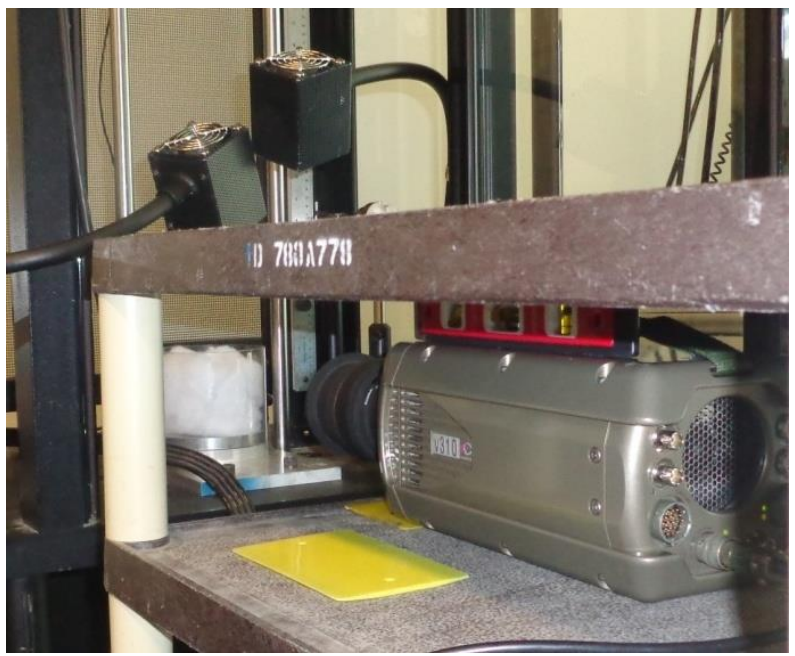

Figure 4.8 High-speed camera used in drop test experiments. 


\subsubsection{Drop test results}

The complete results of the drop tests are shown in Appendix A. Results for a given set of parameters are consistent from experiment to experiment. In the high rate tests, larger forces and thus higher stresses are seen, as expected. Interestingly, however, the difference between high and low density samples at the high strain rate is smaller than anticipated, potentially indicating the onset of the dominance of viscous mechanisms associated with entrapped air near that applied strain rate. It is important to note that in the drop tests the crosshead velocity, and thus the strain rate, is changing continuously throughout the test. Also, importantly, once the kinetic energy of the crosshead reaches a value of zero, the forces acting on the crosshead cause it to reverse direction. The consequence of this is that the fiberfill is not highly compacted prior to the crosshead changing directions. In fact, the fiberfill was not compacted above a nominal strain of $70 \%$ in any of the drop tests. A very large crosshead mass could be used to offset these effects, but would come at the cost of an amplification in the error associated with the force calculation, and is also practically limited by the size of the weights affixed to the crosshead. Also of note is the clear trend of the stress versus strain curves with increasing initial velocity, indicating the activation of rate effects. 


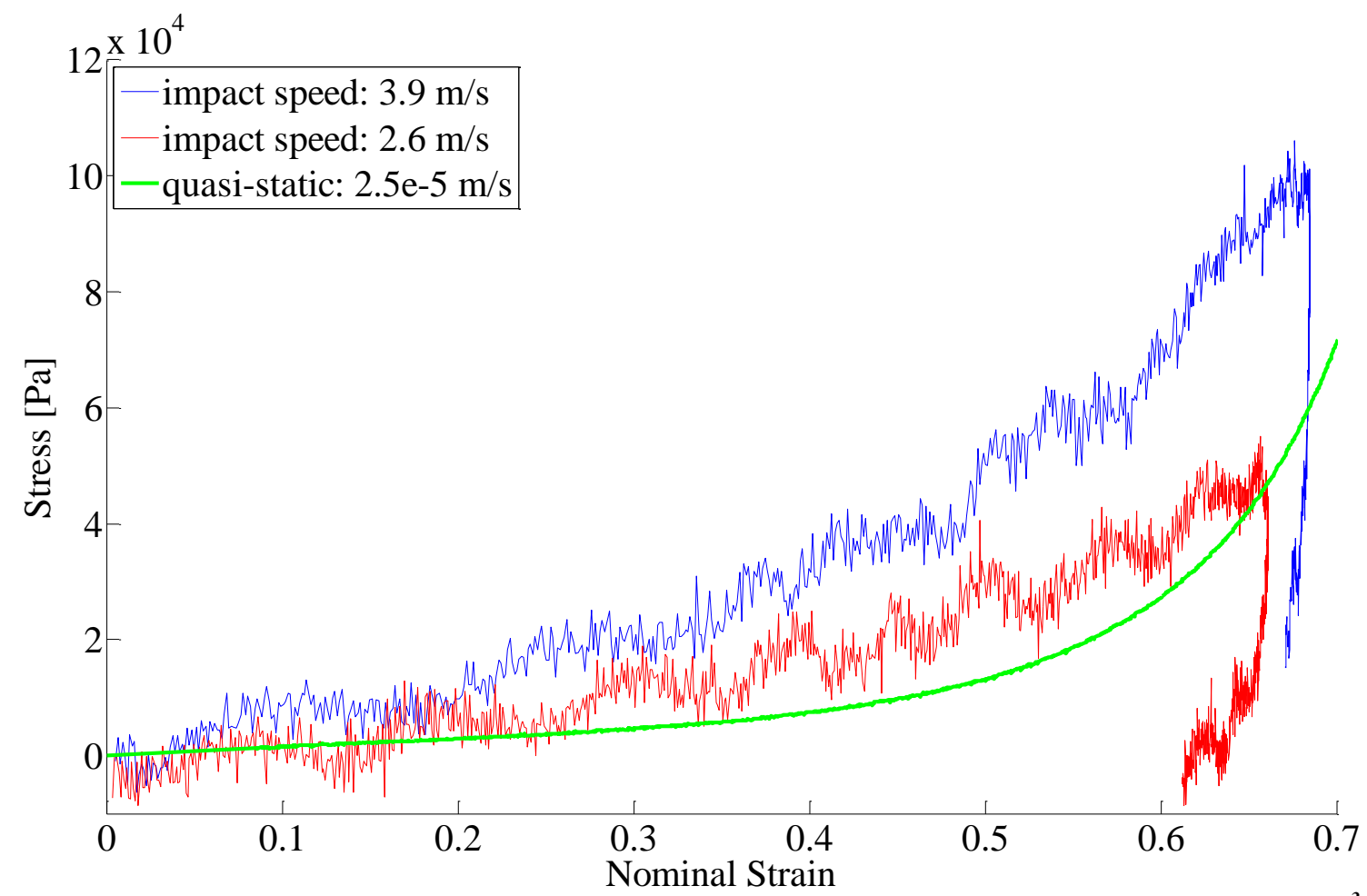

Figure 4.9 Stress versus Nominal Strain curves for differing cross head speeds, Density $=66.7 \mathrm{~kg} / \mathrm{m}^{3}$ for all samples 


\subsection{Tension}

\subsubsection{Micromechanics of fiberfill tension}

Tension appeared to be a dominant mode in the initial ballistic tests. Once the fibers reorient themselves in the direction of the applied load they unfurl and stretch in tension, all the while sliding along their neighboring fibers. The study of the tensile micromechanics of randomly oriented fibers is derived heavily from the theory developed by Cox [57]. Cox theoretically derived the stiffness tensor for an assemblage of straight fibers using an expansion of spherical harmonics of the orientation distribution function. In his 1952 paper Cox also derives the means of load transfer between fibers, a theory now referred to as shear lag in composite materials. Narter et al extended Cox's theory to arbitrary orientation distributions and showed that in the case of random orientation, their theory reduced to Cox's [58]. The predicted elasticity tensor, is however, only applicable to straight fibers, whereas in fiberfill the individuals fibers have significant waviness due to their multiple crimps. Crimped fibers must unfurl before they are able to obtain the stiffness of a straight fiber with the same cross section and elastic properties. This leads to marked nonlinearity in the tensile response of entangled fibers, and is the topic of a significant quantity of research in recent years with applications in the field of biological tissues and micromechanical modeling of polymers [59] to [62]. In fact, fiberfill is not unlike a collagen fiber reinforced biological tissue, however it is lacking in a matrix material and thus the individual fibers are much more mobile. A number of different force-stretch relationships have been derived to model the behavior of collagen fibers including the Freely Jointed Chain, Wormlike Chain, and Euler elastica based formulations [63].

\subsubsection{Tension experimental setup and procedure}

Tensile specimens were cut, from a large fiberfill roll (Figure 4.10), into strips with widths of 40 to $50 \mathrm{~mm}$ and lengths of $\sim 90 \mathrm{~mm}$. These specimens were then adhesively bonded to $1 \mathrm{~mm}$ thick aluminum sheets, the adhesive and fibers forming a composite core sandwiched between the 
aluminum (Figure 4.11). This allowed the load to be transferred from the grips of the testing machine to the fibers in the specimens in a fairly uniform manner. For testing, the specimens were placed in a screw-driven Instron ${ }^{\circledR}$ universal test frame equipped with a $500 \mathrm{~N}$ load cell. The samples were tested at low rates until complete failure. Some specimens of higher than nominal density were prepared by tightly rolling larger pieces of fiberfill, which were then bonded to aluminum sheets in the same manner as the low density samples. Samples cut from the roll direction, in addition to the cross direction, were also tested to investigate initial anisotropy in the fiberfill. A summary of the tensile test parameters is given in Table 4.4.
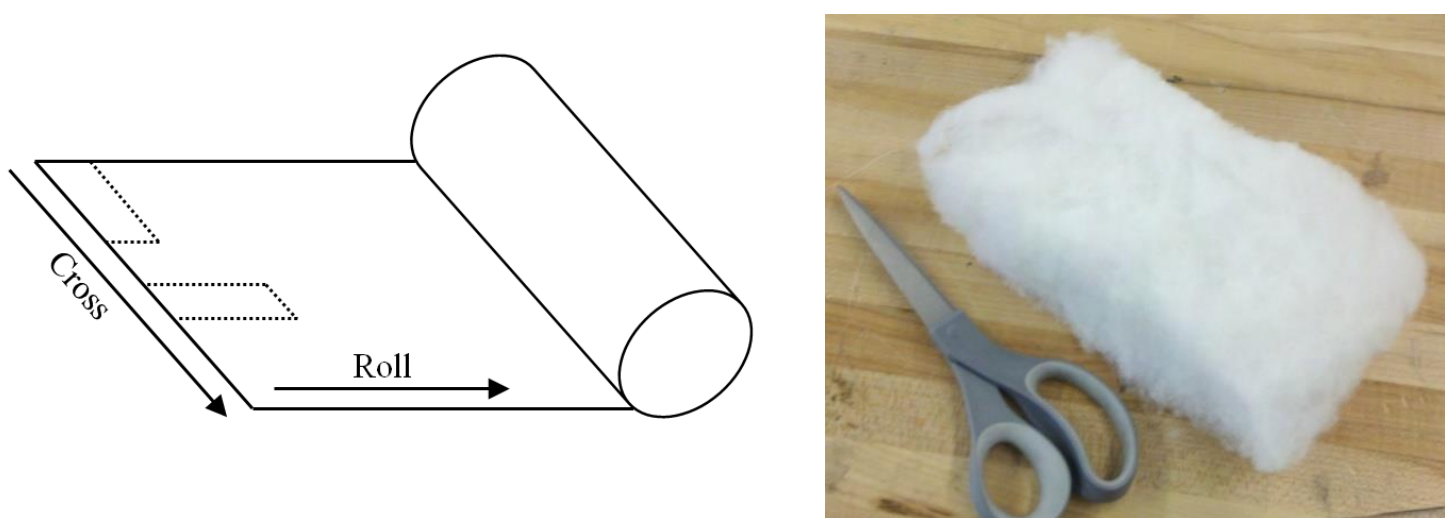

Figure 4.10 Fiberfill sample preparation 


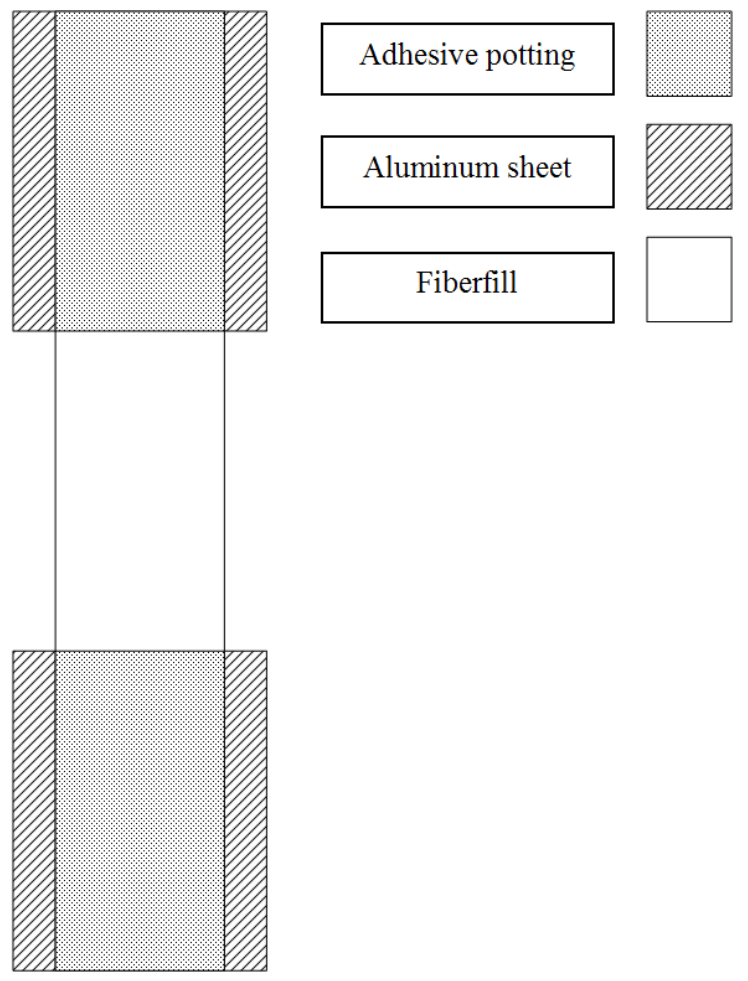

Figure 4.11 Fiberfill Tensile Specimen

Table 4.4 Parameters for tension experiments

\begin{tabular}{|c|c|c|c|}
\hline Test ID & $\begin{array}{c}\text { Relative } \\
\text { Density }\end{array}$ & $\begin{array}{c}\text { Strain rate } \\
{[\mathbf{1} / \mathbf{s}]}\end{array}$ & Direction \\
\hline 001 & $1.38 \mathrm{E}-02$ & $9.06 \mathrm{E}-04$ & Cross \\
\hline 002 & $9.07 \mathrm{E}-03$ & $9.06 \mathrm{E}-04$ & Cross \\
\hline 003 & $1.29 \mathrm{E}-02$ & $3.62 \mathrm{E}-03$ & Cross \\
\hline 004 & $8.86 \mathrm{E}-03$ & $3.62 \mathrm{E}-03$ & Cross \\
\hline 005 & $8.87 \mathrm{E}-03$ & $1.09 \mathrm{E}-02$ & Cross \\
\hline 006 & $8.22 \mathrm{E}-03$ & $4.35 \mathrm{E}-02$ & Cross \\
\hline 007 & $9.22 \mathrm{E}-03$ & $1.53 \mathrm{E}-04$ & Cross \\
\hline 008 & $1.55 \mathrm{E}-02$ & $1.38 \mathrm{E}-03$ & Cross \\
\hline 009 & $3.16 \mathrm{E}-02$ & $1.31 \mathrm{E}-03$ & Cross \\
\hline 010 & $5.83 \mathrm{E}-03$ & $1.27 \mathrm{E}-03$ & Roll \\
\hline 011 & $6.08 \mathrm{E}-03$ & $1.15 \mathrm{E}-03$ & Roll \\
\hline 012 & $1.06 \mathrm{E}-02$ & $1.61 \mathrm{E}-03$ & Cross \\
\hline 013 & $8.80 \mathrm{E}-03$ & $1.61 \mathrm{E}-03$ & Roll \\
\hline 014 & $8.33 \mathrm{E}-03$ & $1.61 \mathrm{E}-03$ & Roll \\
\hline
\end{tabular}




\subsubsection{Tension results}

In the initial stage of deformation the locked-in stress, due to static friction between fibers, dominates and the stress is due almost entirely to the stretching and bending of fibers without reorientation. The response in this low strain regime appears to follow a power-law like curve for all samples tested. After this very small elastic regime tensile deformation was dominated by the rotation and unfurling of the crimped fibers. The realignment of fibers in the loading direction led to a Poisson-like effect with samples showing significant reductions in cross section. As the load increased, fibers began slipping from their entanglements. After the peak load was reached, the deformation was dominated by gross slippage of fibers, which ultimately led to the softening of the response for higher levels of strain. Near the end of each test, damage localized in a small region near the middle of the sample and total failure occurred. An example of these stages of deformation is shown in Figure 4.12 and Figure 4.13.

Samples tested in the roll direction showed approximately the same initial stiffness as those cut in the cross direction, but failed at much lower stresses. This is due to the alignment of the fibers induced by the manufacturing process, with preferential alignment in the cross direction [41], [42]. 


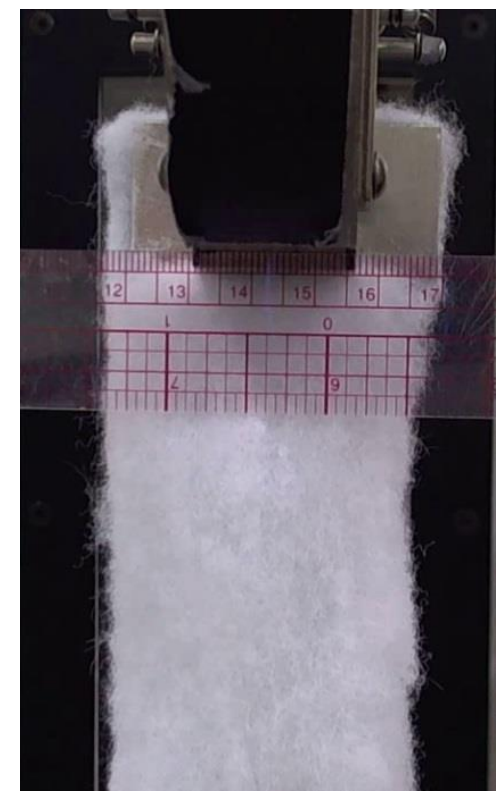

A: Start of test

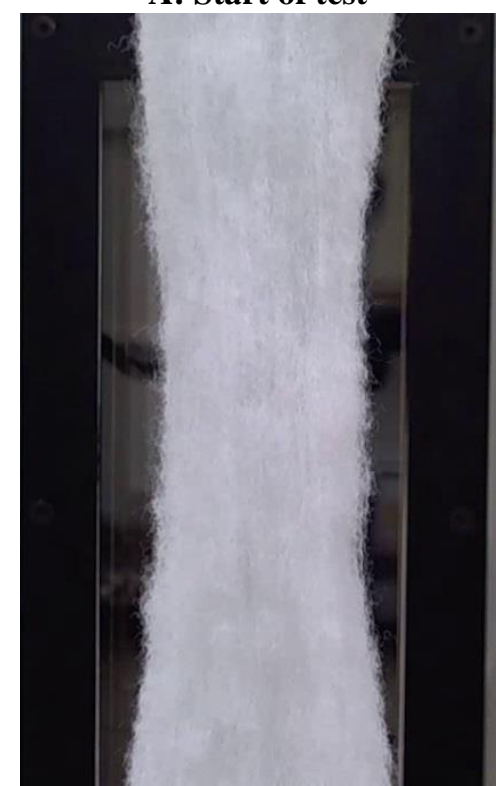

D: Uniform damage progression

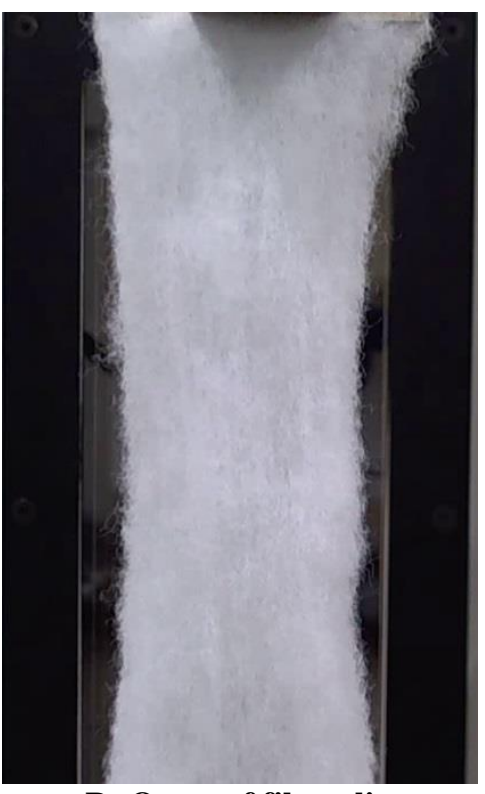

B: Onset of fiber slip

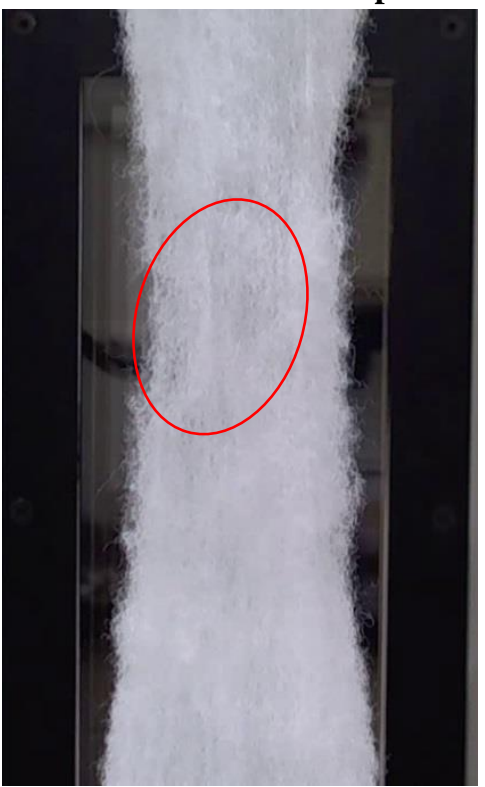

E: Damage localization

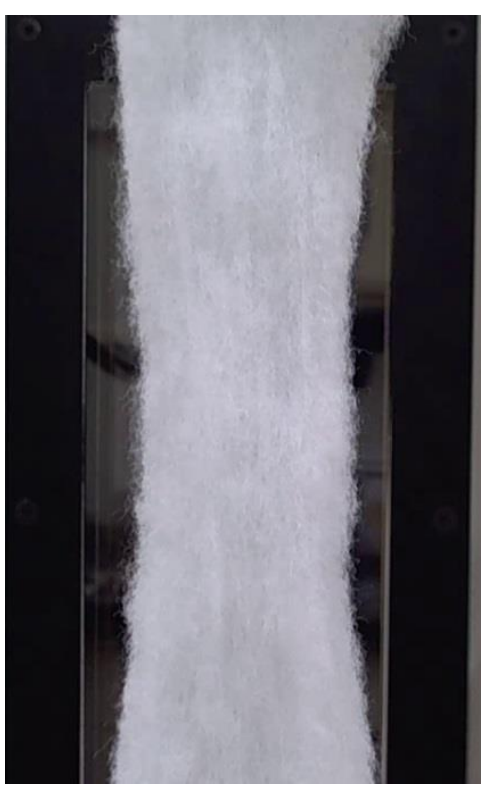

C: Load plateau

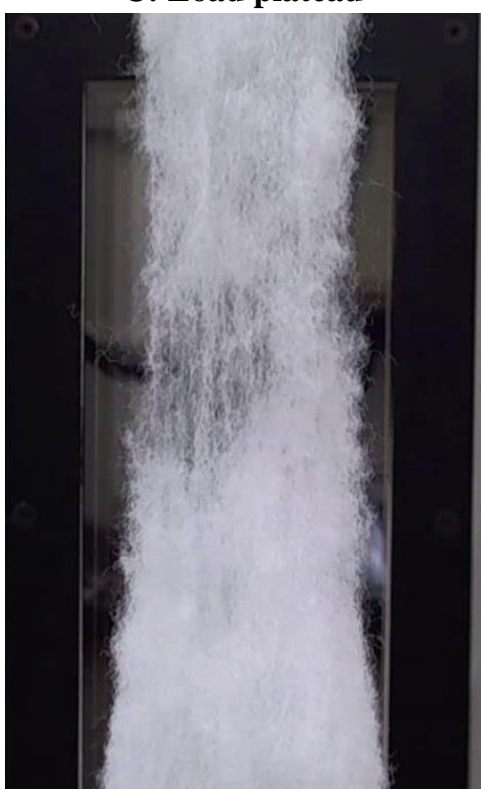

F: End of Test

Figure 4.12 Images from tensile test 007. See Figure 4.13 


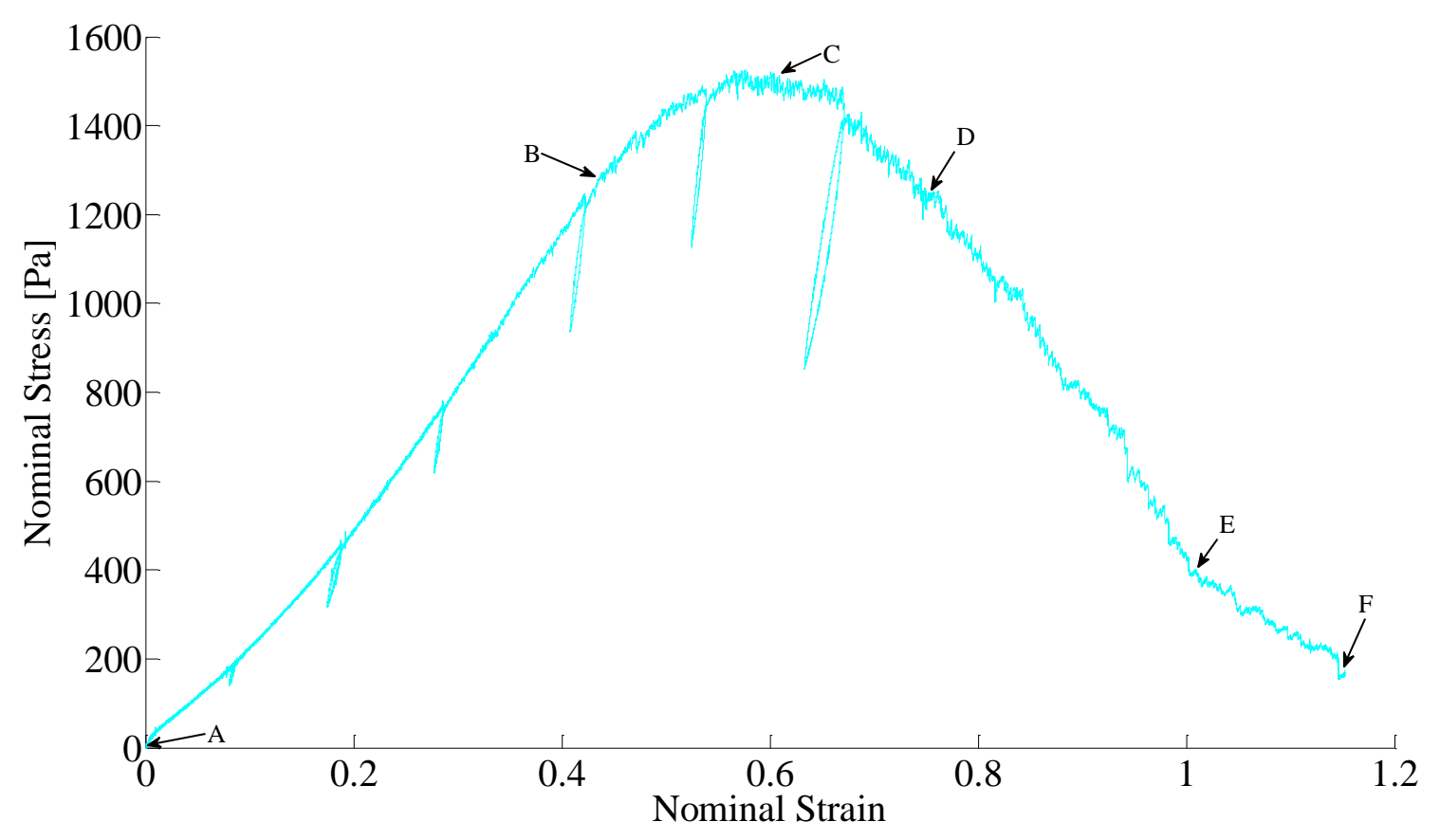

Figure 4.13 Tensile test 007; several load-unload cycles shown

There was significant variation in the stress-strain behavior of the tensile specimens, even among samples with very similar densities. Much of this variability may be due to the inherent variability within the material. Another source of variability may be due to the uncertainty of width and length measurements. This may have also been due to handling of the fiberfill while preparing the samples, which introduced variable levels of inelastic deformation to the different samples. It is also likely that the starting strains of the samples were not equal, which affected the measured stiffness.

A comparison of sample IDs 003, 004, and 005 is shown in Figure 4.16 and Figure 4.17. Sample 003 had a very similar relative density to that of sample 008, but showed significantly lower strength and initial stiffness. Sample 009 had the highest strength and initial stiffness, and showed an extended power law region (Figure 4.17), indicating a higher degree of locked in friction and lower fiber mobility. Comparisons of peak load and initial strength are shown in Figure 4.18 and Figure 4.19 respectively. 


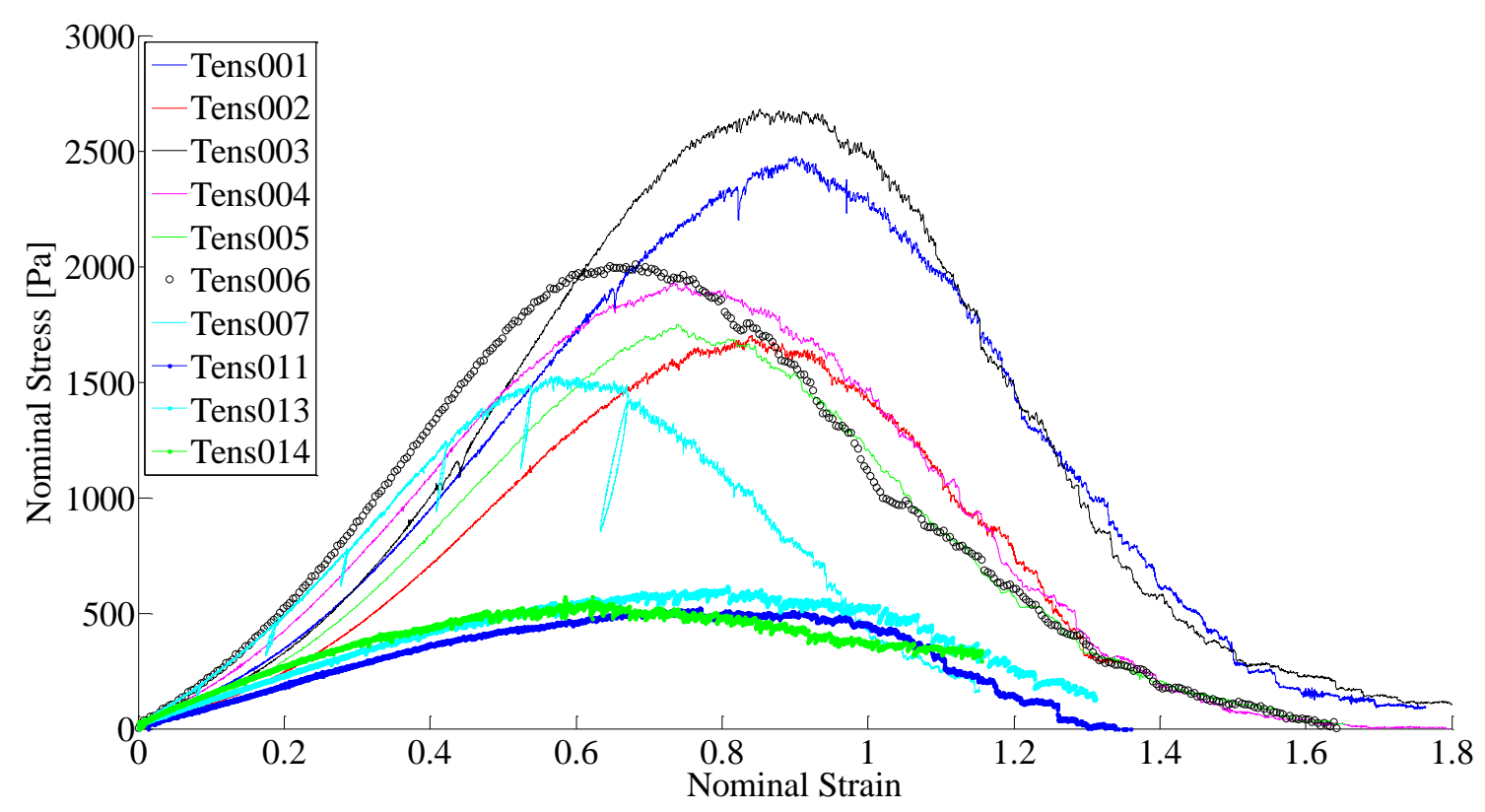

Figure 4.14 Tensile data; low density samples

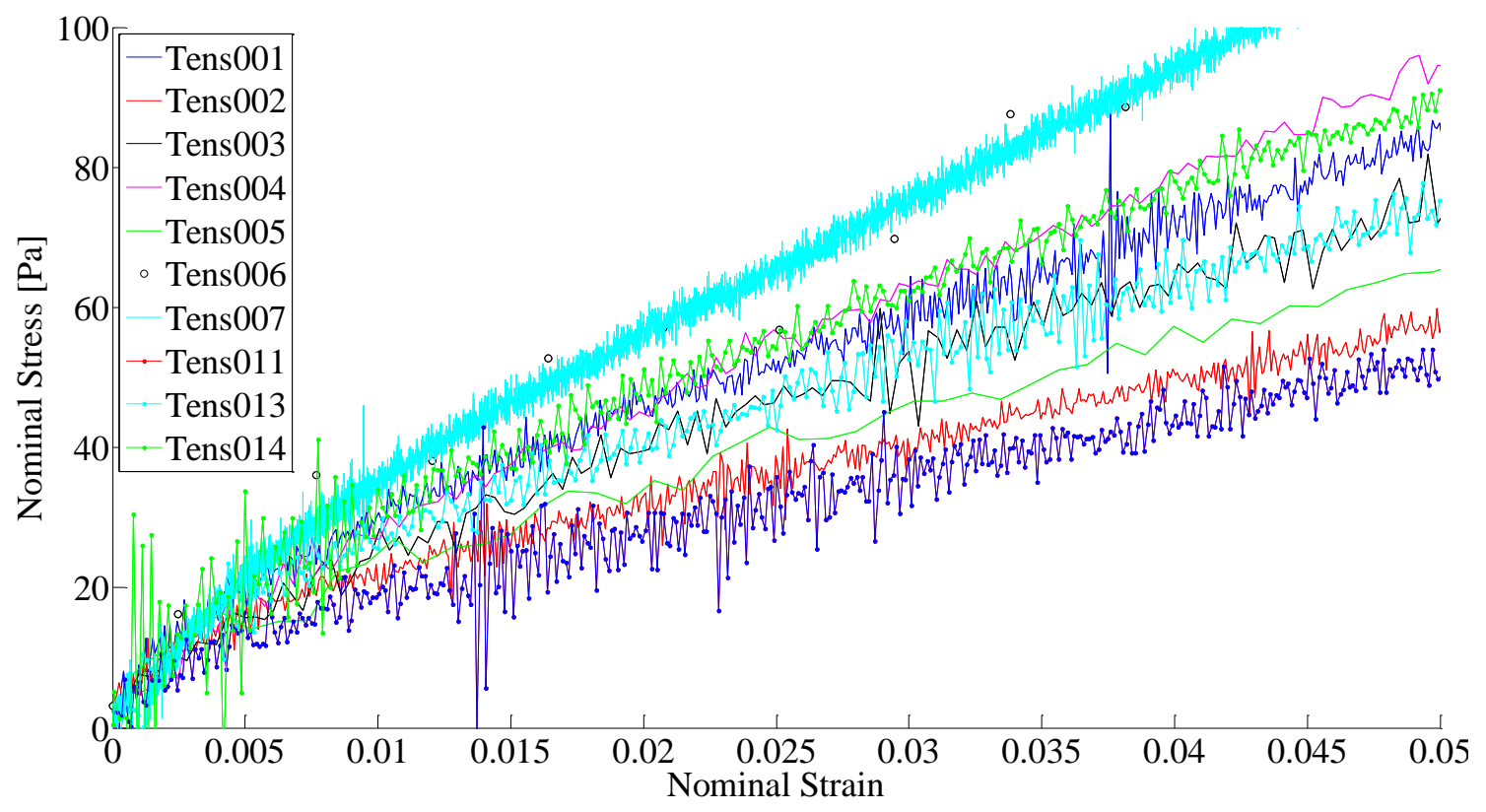

Figure 4.15 Tensile data; low density samples, small strain regime 


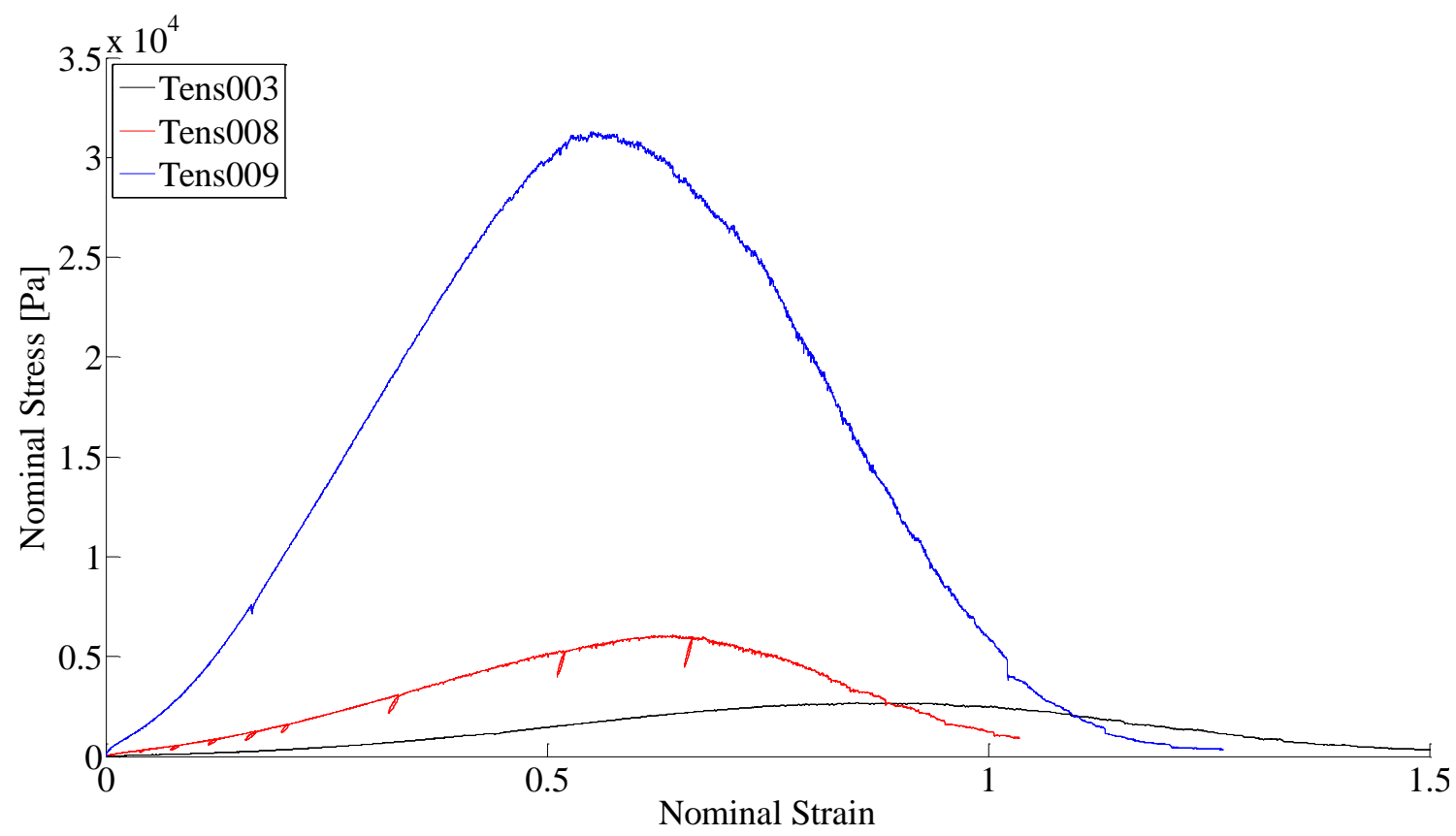

Figure 4.16 Comparison of high (009), medium (008) and low (003) density tensile samples

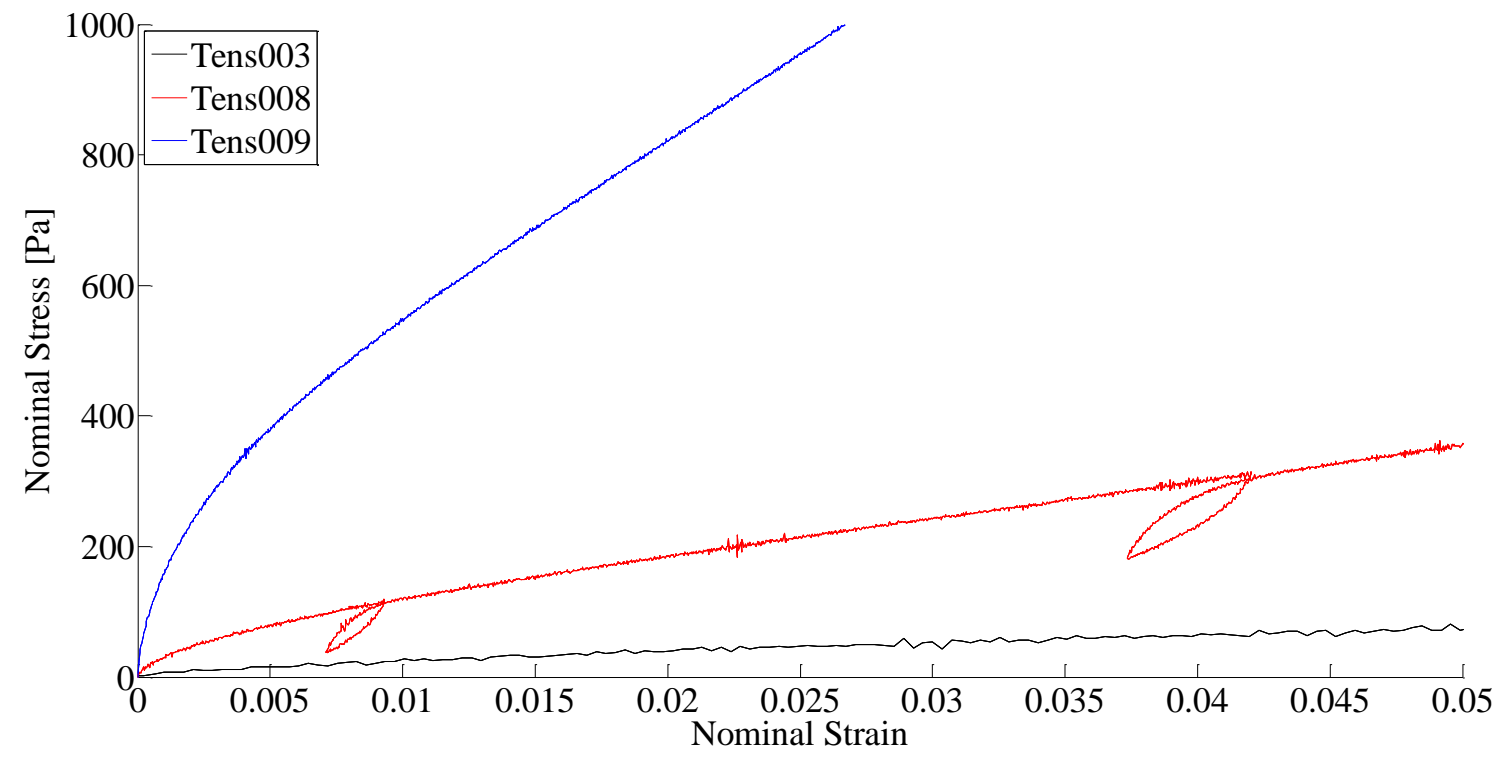

Figure 4.17 Comparison of high (009), medium (008) and low (003) density tensile samples; small strain regime 


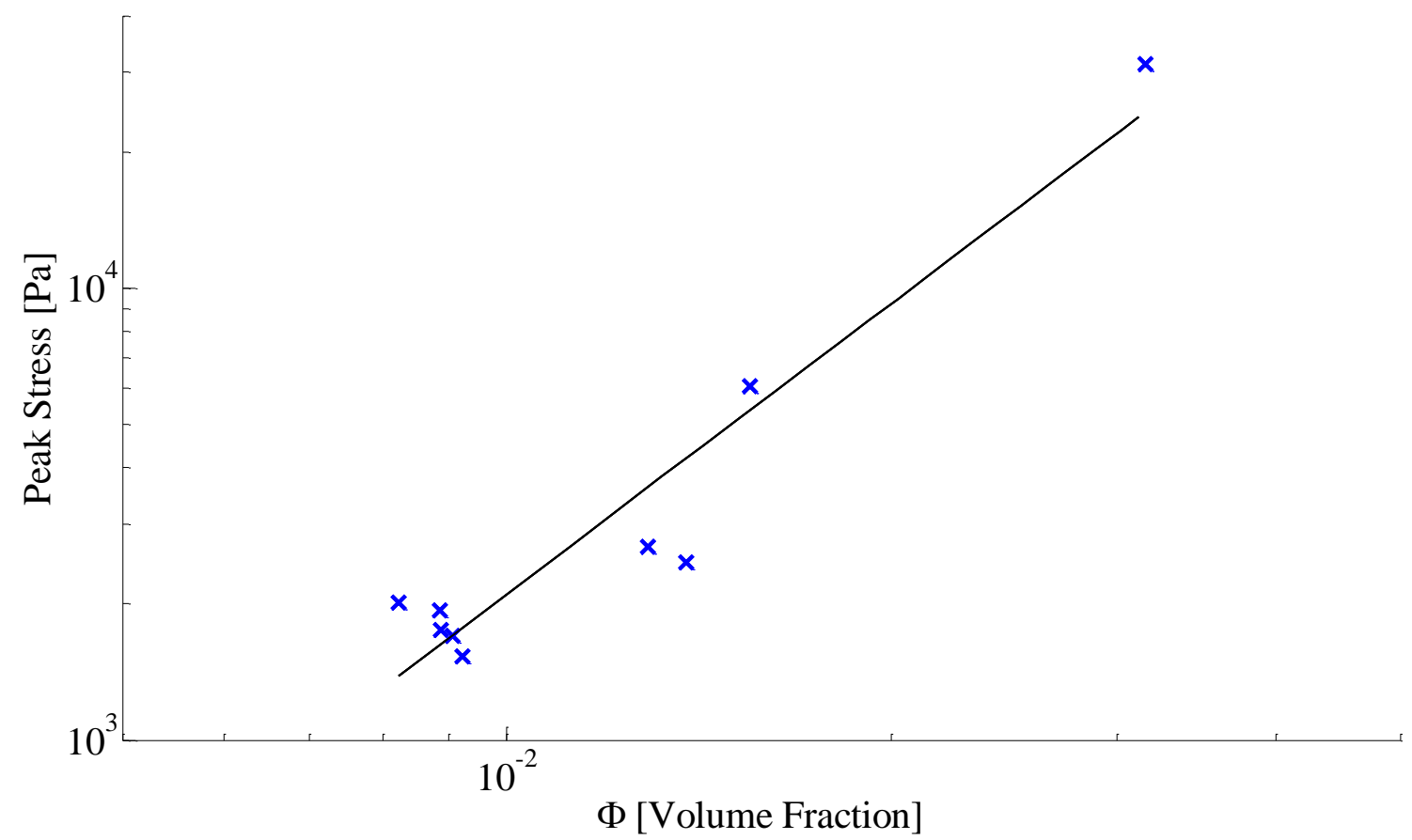

Figure 4.18 Peak stress versus volume fraction; cross direction samples. Power law fit shown in black

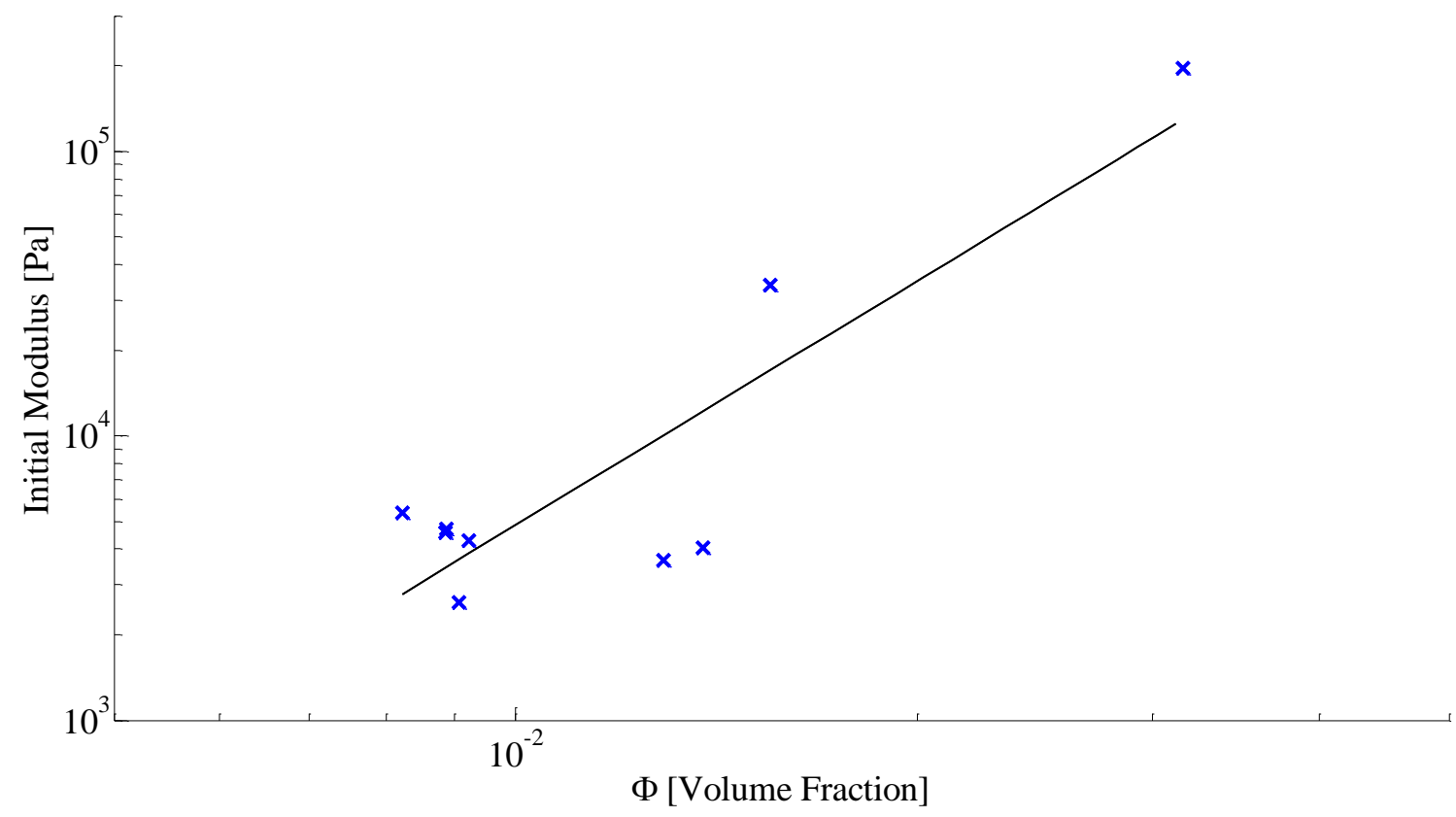

Figure 4.19 Initial modulus versus volume fraction; cross direction samples. Power law fit shown in black 
The curve fits shown for these data sets are:

$$
\begin{gathered}
\sigma_{\text {peak }}=3.90 \mathrm{e} 7 \times \Phi^{2.134}[\mathrm{~Pa}] \\
\mathrm{E}_{\text {initial }}=2.46 \mathrm{e} 9 \times \Phi^{2.852}[\mathrm{~Pa}]
\end{gathered}
$$

Eq 4.7

Eq 4.8

indicating a strong dependence on fiber volume fraction. For comparison, the modulus of opencelled foams varies according to: $\mathrm{E}_{\text {foam }}=\mathrm{E}_{\text {solid }} \Phi^{2}$ where $\mathrm{E}_{\text {solid }}$ is the modulus of a solid piece of material from which the foam is made $\left(\mathrm{E}_{\mathrm{PET}} \approx 2 \mathrm{e} 9[\mathrm{~Pa}]\right)$. The relatively high exponent for fiberfill has two implications: first, that fiberfill is bending dominated in small-strain tension, and second, that the mechanical performance of fiberfill has a higher sensitivity to volume fraction than foam made of the same parent material [55]. 


\subsection{Shear}

\subsubsection{Micromechanics of fiberfill Shear}

Shear micromechanics are very similar to those of tension, with unfurling, fiber realignment, and interfiber slip all playing a part in the deformation. Skelton studied the effects of friction on the shear behavior of polyethylene felts [64]. He determined that a simple power law equation could describe the small strain regime of both bending and shear deformation, with increasing strain leading to a decreased tangent shear modulus due to interfiber slip. Pan and Carnaby later analyzed the small strain shear deformation of fibrous assemblies and determined elastic constants for a random assembly of straight fibers [65]. They assumed that the two dominant mechanisms were fiber bending between contact points, and fiber slip. In both analyses the contact point density and contact-point-to-contact-point distance play strong roles in dictating the deformation behavior.

\subsubsection{Shear experimental setup and procedure}

Shear specimens were made by adhesively bonding strips of fiberfill to plates which were mounted in a tensile plate shear fixture designed per ASTM C273. The specimens were tested to failure at strain rates between $3.28 \mathrm{e}-4$ to $3.28 \mathrm{e}-3[1 / s]$. The parameters for each shear test are listed in Table 4.5

Table 4.5 Parameters for shear experiments

\begin{tabular}{|c|c|c|}
\hline TestID & Relative Density & Strain rate [1/s] \\
\hline 001 & $7.87 \mathrm{E}-03$ & $3.28 \mathrm{E}-04$ \\
\hline 002 & $7.54 \mathrm{E}-03$ & $3.28 \mathrm{E}-03$ \\
\hline 003 & $6.80 \mathrm{E}-03$ & $1.64 \mathrm{E}-03$ \\
\hline 004 & $7.75 \mathrm{E}-03$ & $3.28 \mathrm{E}-03$ \\
\hline 005 & $8.02 \mathrm{E}-03$ & $3.28 \mathrm{E}-03$ \\
\hline 006 & $8.09 \mathrm{E}-03$ & $3.28 \mathrm{E}-03$ \\
\hline
\end{tabular}




\subsubsection{Shear results}

The shear behavior of fiberfill was very similar to its tension behavior; with an initial high stiffness region, corresponding to the locked-in frictional mechanism, followed by softening as fibers began to slide. In the large strain regime, fiber realignment in the maximum shear stress direction and fiber unfurling led to a stiffening of the response up to about $100 \%$ strain. After $\sim 100 \%$ strain fiber slippage from junction points began to dominate, and significant softening occurred. The fiberfill was, however, able to achieve engineering shear strains of 350 to $500 \%$ prior to complete failure. Again, very little rate effect was seen in the range of strain rates tested. Photographs of a shear test are shown in Figure 4.20 and Figure 4.21. Shear results are summarized in Figure 4.22 and Figure 4.23.

Again, considerable variation in the stress-strain behavior was observed. The shear specimens underwent a significant amount of handling during sample preparation, thus increasing the risk of inducing permanent deformation in the samples. In addition, the specimens were somewhat compressed from their initial state during the adhesive bonding process, which likely affected the outcome of the shear tests. 


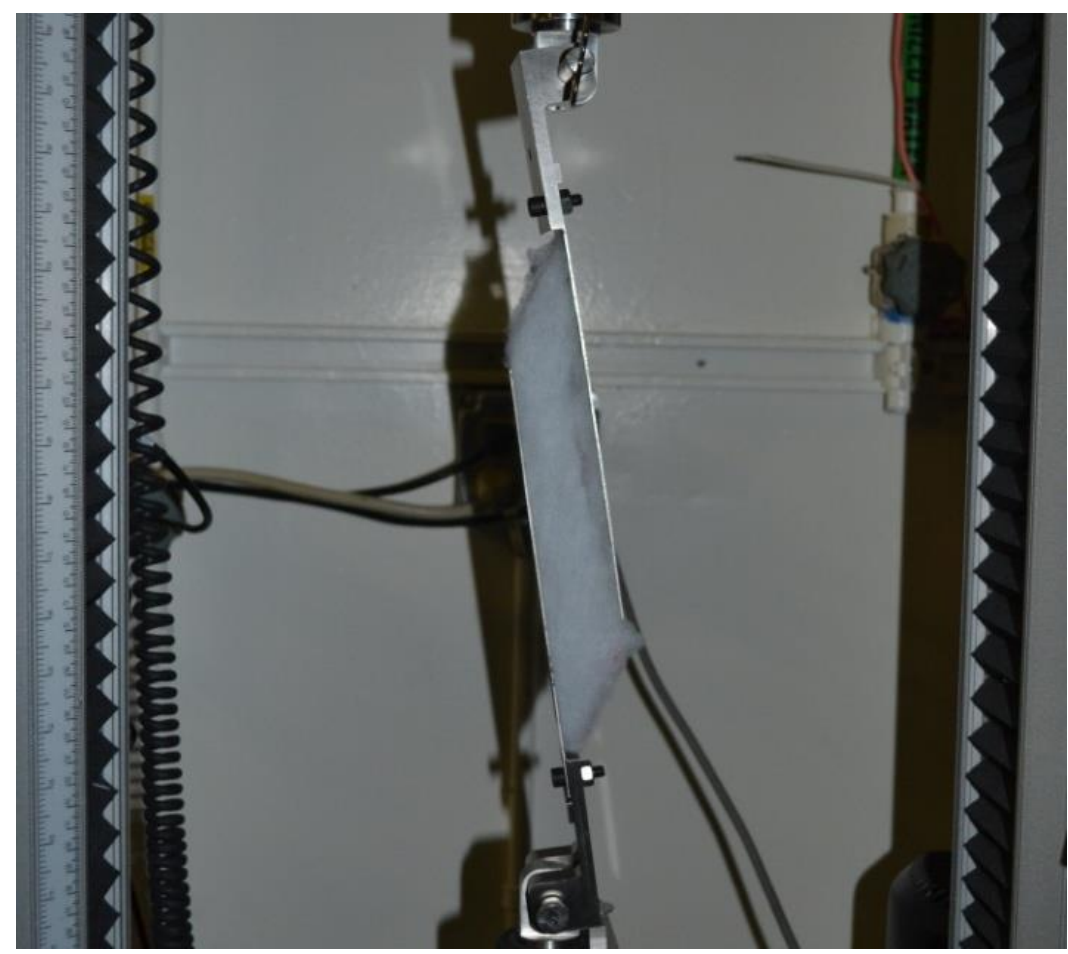

Figure 4.20 Shear specimen 005 at $100 \%$ shear strain.

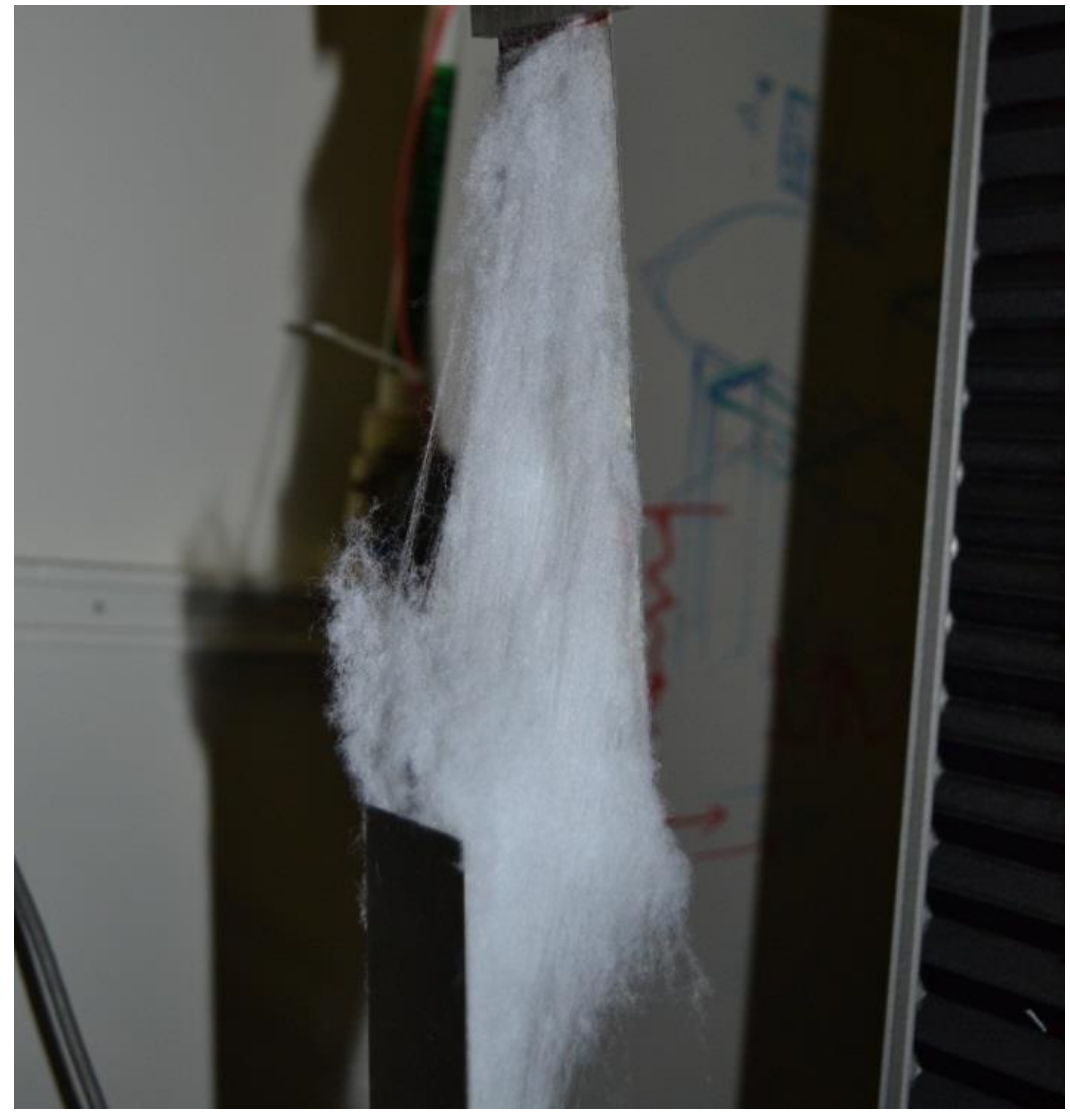

Figure 4.21 Shear specimen 005 near end of test. 


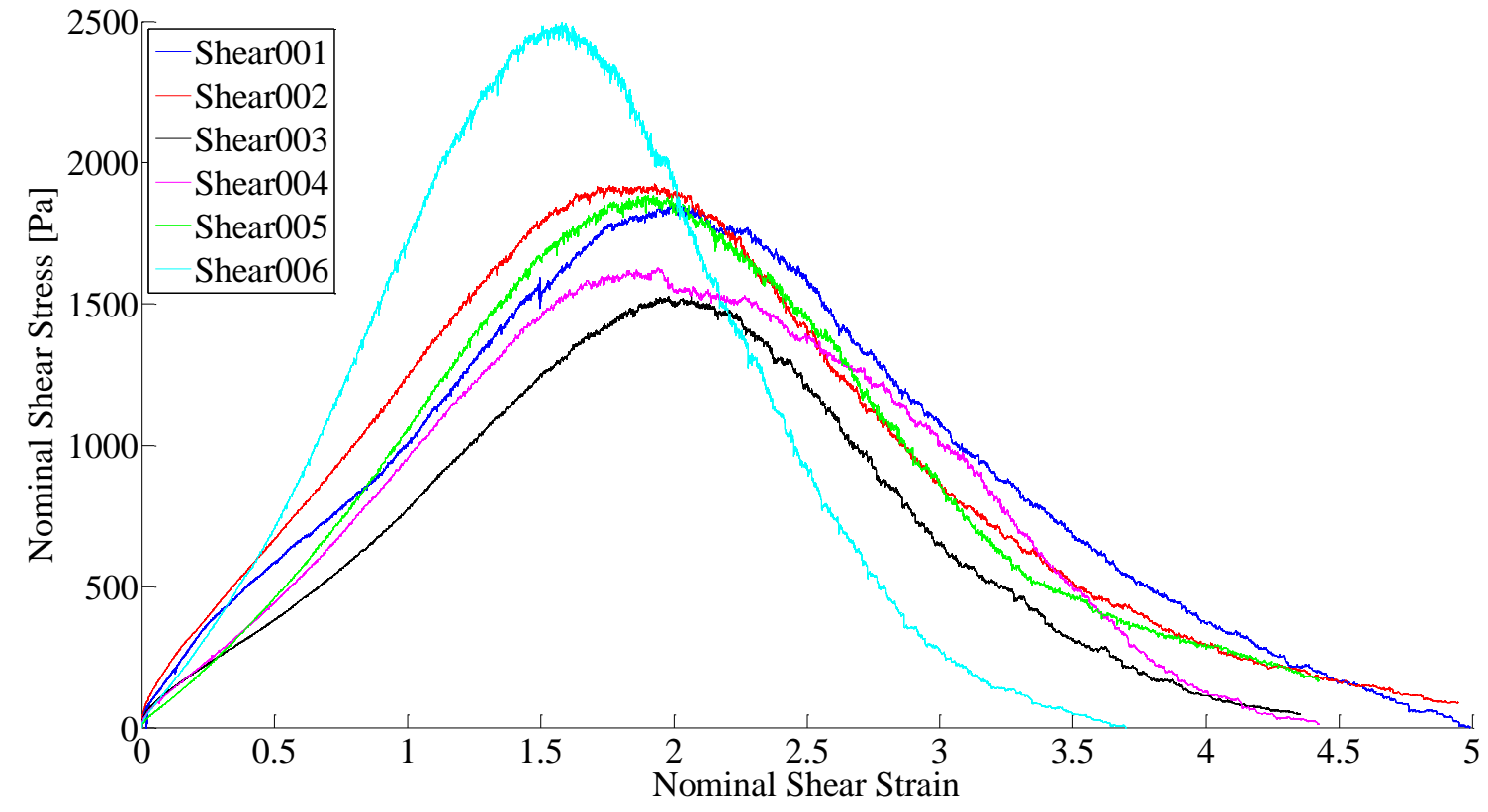

Figure 4.22 Shear data

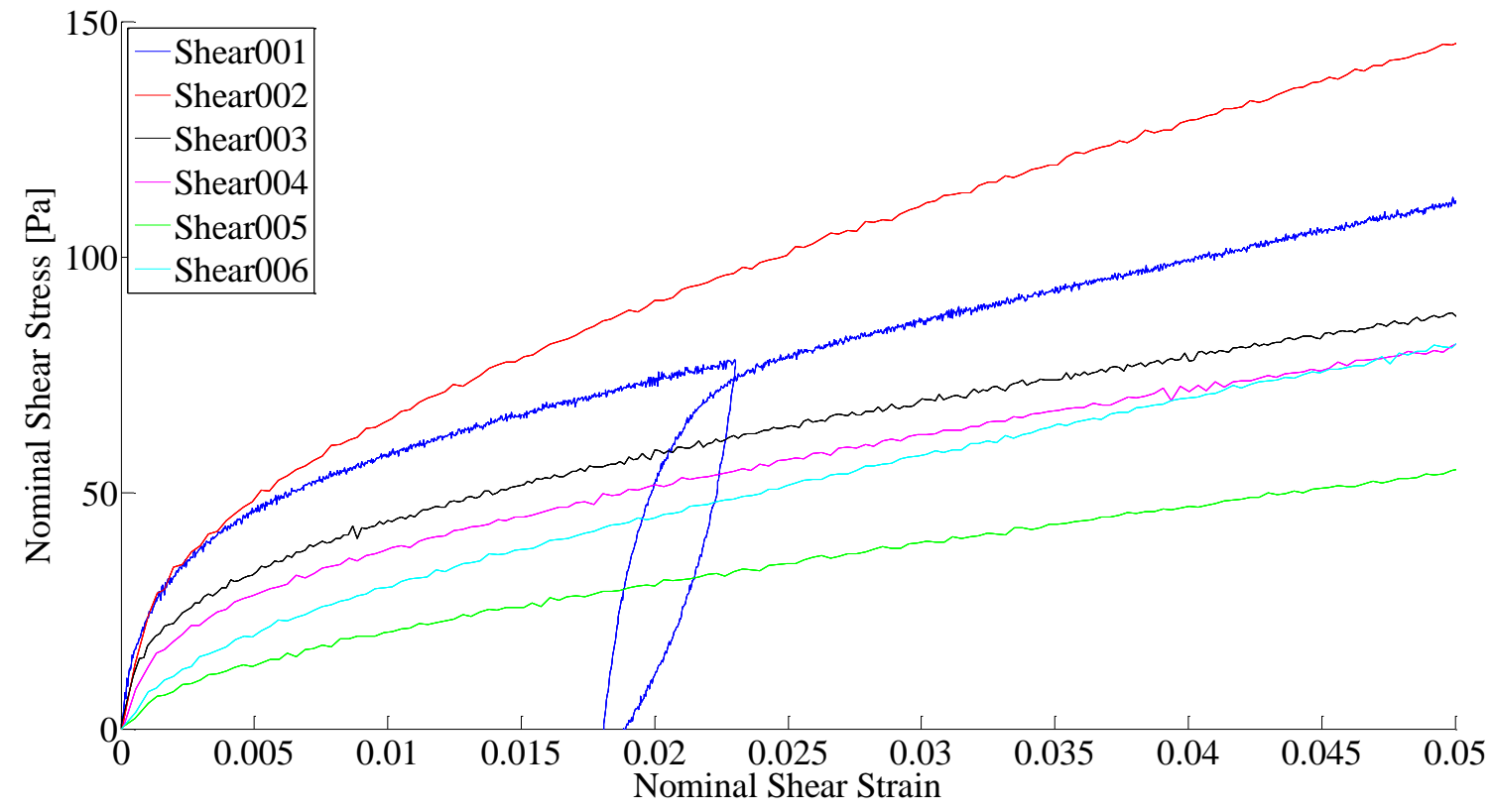

Figure 4.23 Shear data; small strain regime 


\section{Gas Gun Tests}

\subsection{Experimental procedure and test fixture}

Gas gun tests were conducted in the mechanical testing facilities at the University of California Santa Barbara [5], [12]. The light gas gun utilized in this testing used high pressure nitrogen as a propellant, and had swappable barrels of either 7.62 or $45 \mathrm{~mm}$ bore diameters (Figure 5.1 and Figure 5.2). The gun has the ability to fire low velocity shots (100 to $450 \mathrm{~m} / \mathrm{s}$ ) using just the breech chamber, or can fire high velocity shots $(\sim 1000 \mathrm{~m} / \mathrm{s})$ using the breech chamber in conjunction with a larger charge chamber. Experiments conducted in this thesis used only the low velocity configuration of the gas gun.

The gun is loaded from the breech end and can use a variety of different projectiles. The projectiles used were: $7.54 \mathrm{~mm} 304$ stainless steel ball bearings (BB), $7.54 \mathrm{~mm}$ diameter by 40 $\mathrm{mm}$ long 6061-T6 aluminum fragment simulating projectiles (FSP), and $44.5 \mathrm{~mm}$ diameter $75 \mathrm{~mm}$ long 6061-T6 aluminum slugs. In the small barrel, a tiny gap exists between the outer dimension of the projectile and the bore of the barrel. A urethane foam obturator was taped to the back of each projectile. The obturator was cut slightly oversized in order to form an interference fit with the barrel, thus preventing high pressure propellant gas from bypassing the projectile.

A piece of brass shim stock separates the breech chamber from the barrel. To fire the gun, pressure in the breech is slowly increased until the brass shim ruptures; allowing the high pressure propellant gas to expand, which then forces the projectile down the barrel. Coarse adjustments of the velocity can be made by using thinner or thicker brass shims, thus altering the burst pressure. Fine velocity adjustments are accomplished by shifting the location of the projectile either closer to the muzzle of the barrel to reduce the velocity, or closer to the breech to increase the velocity. An array of laser gates is mounted in the muzzle of the barrel for measuring 
the projectile's speed. When the beams formed by these gates are interrupted by the passing projectile, the signal is recorded on a digital oscilloscope and the muzzle velocity can be determined from the delay between gate triggers. The laser gate signals trigger the high speed camera recording and trigger a high intensity flash to provide sufficient lighting for the camera footage. Tuning of the delay and ramp times for both the flash and camera is necessary to obtain good footage from each shot. Exit velocities, where applicable, were determined using the location of the projectile in, and the timestamp of, a series of frames taken after the projectile has exited the back side of a sample under test.

Fiberfill was stuffed into transparent polycarbonate constraint tubes and mounted to a fixture at the muzzle of the gas gun. In the BB and FSP tests, fiberfill areal densities ranging from 4.4 to $10.7 \mathrm{~kg} / \mathrm{m}^{2}$ were tested. The highest of these densities was tested in four different tube diameters ranging from 47.6 to $197 \mathrm{~mm}$ to determine if there was a "near wall" effect in the smaller tube diameters. Difficulty was encountered with keeping the fiberfill in the constraint tube throughout the duration of the test. The projectile, the air in the barrel ahead of the projectile, and the highpressure gas behind the projectile all had a tendency to plug out the entire fiberfill sample. To overcome this issue, samples were sealed into their constraint tubes using a two-ply layer of duct tape on the each end of the tube. This proved to still be insufficient at keeping the entire sample from plugging out of the constraint tube, so a backing plate of $0.5 \mathrm{~mm}$ thick 2024 aluminum with a $25.4 \mathrm{~mm}$ orifice, roughly concentric with the sample under test, was added. The contribution of the duct tape and orifice plate to the ballistic performance was neglected. The orifice plate limited the back facing duct tape's ability to membrane-stretch and served to limit the quantity of fiberfill participating in slowing projectiles as they exited the back of the samples, which is assumed to be a negative performance effect offsetting the increase in areal density from the duct tape.

A large steel tube filled with alternating layers of ballistic sand and high density foam, and backed by a thick steel plate was placed behind each sample at a small standoff distance. This 
"catch can" had a layer of duct tape placed across its face, both acting to hold its sand and foam contents in place, and serving as a witness plate. If the outer layer of duct tape was ruptured in any way, the shot was deemed an armor defeat as per MIL-STD-662-F. $\mathrm{V}_{50}$ was determined by taking the average of the two highest non-defeating shot velocities with the two lowest armor defeating shot velocities. Many shots that exited the sample under test at non-zero velocities were slowed enough to not puncture the witness tape. Thus, the exit velocity of a given projectile proved to be a poor metric for determining the actual $\mathrm{V}_{50}$, since the projectile was continuing to slow as it exited the frame of the camera and headed toward the catch can.

FSP tests were conducted to elucidate any projectile destabilization effects that the fiberfill might have on a cylindrical rather than spherical projectile. These tests were only conducted on the higher density fiberfill. The issue of determining exit velocities in these tests was exacerbated by the destabilization of projectiles near the ballistic limit which tended to cause the projectiles to veer out of the frame.

For comparison testing $101.6 \times 101.6 \mathrm{~mm}, 1.27 \mathrm{~mm}$ thick 1095 blue tempered spring steel, and monolithic $6.35 \mathrm{~mm}$ thick PET sheets were tested. These control samples had approximately the same areal density as the fiberfill. The monolithic sheets were edge clamped in a transparent enclosure directly affixed to the catch can assembly. $V_{50}$ for these comparison samples was determined by the same method utilized for fiberfill.

Alternate fiber materials were also studied. One set of BB experiments was run on a commercial PET fiberfill called Polyfil. Additionally, high performance fiberfill samples made from Kevlar 49 and Spectra 1000 fibers were prepared and tested. Fibers from these two materials were cut into $100 \mathrm{~mm}$ lengths, entangled and fluffed by hand. The process of making these samples by hand was incredibly time consuming, and consequently tests on high performance fiberfill was limited. 
Several tests were performed with a mix of Kevlar and PET fiberfill to determine how the top layer of fiberfill deformed. Samples for these tests consisted of a layer of Kevlar fiberfill backed with a thicker layer of PET fiberfill (Figure 5.3).

Slug tests were performed on fiberfill samples mounted in polycarbonate tubes with an open faced front side and rigid steel backing plate. These tests were intended to approximate the drop test experiments performed earlier, but at higher strain rates.

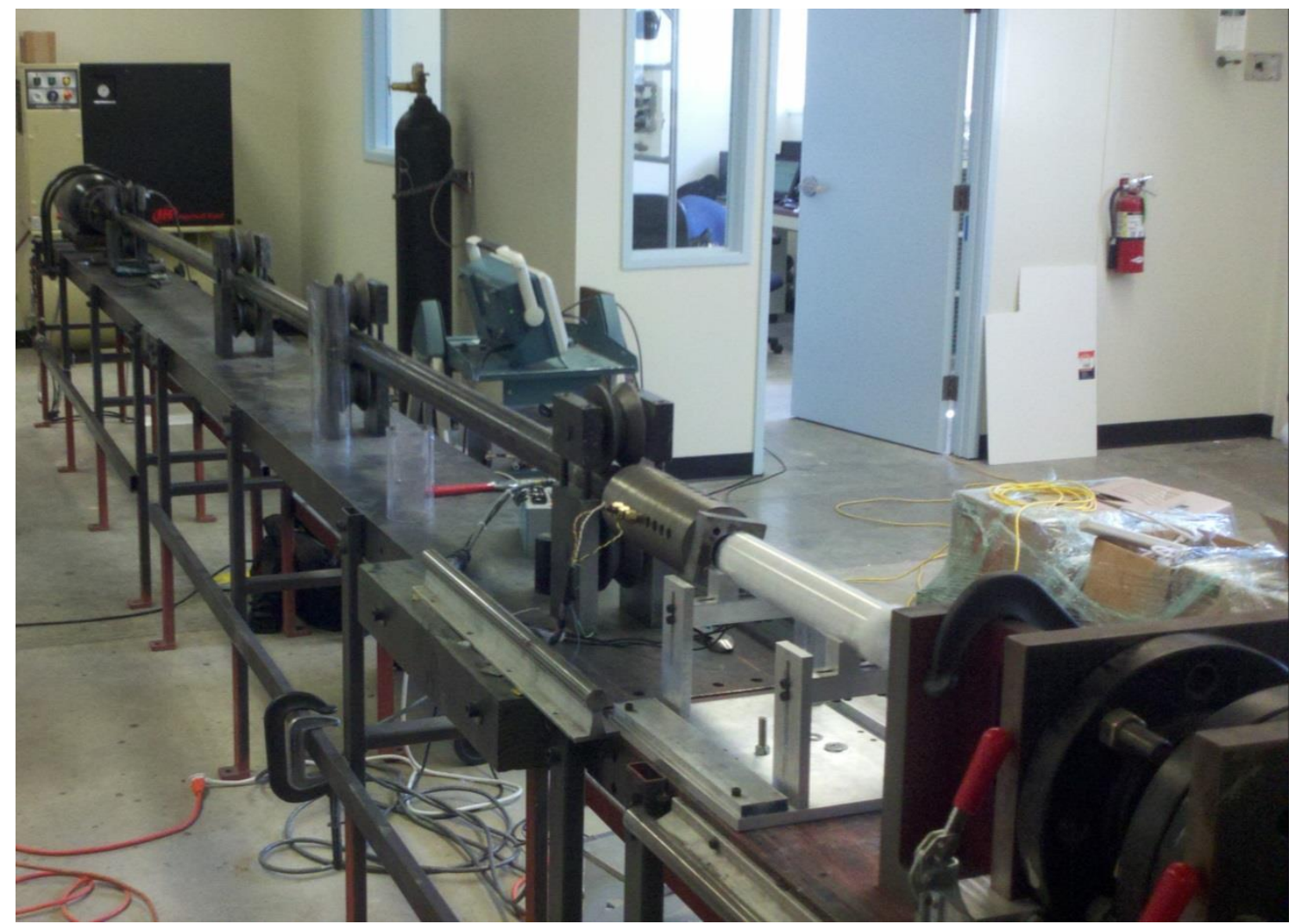

Figure 5.1 Gas gun configured with $45 \mathrm{~mm}$ barrel 


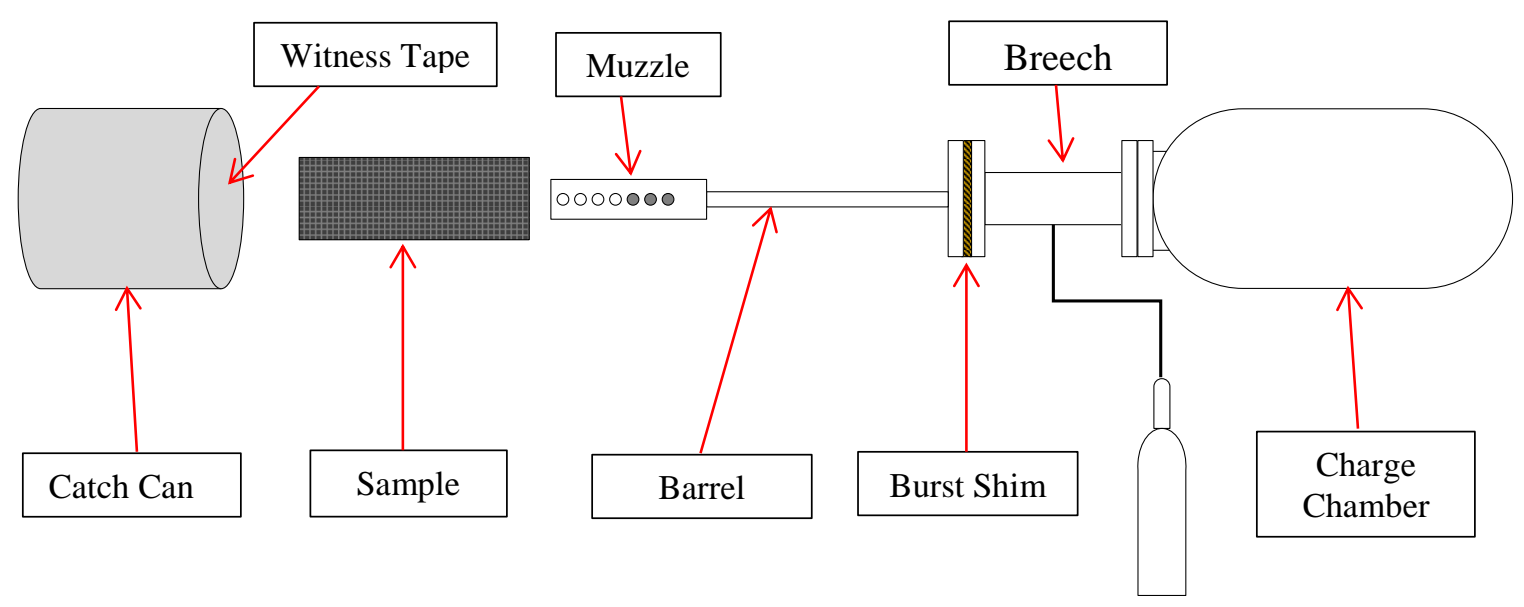

Figure 5.2 Gas gun schematic (not to scale)

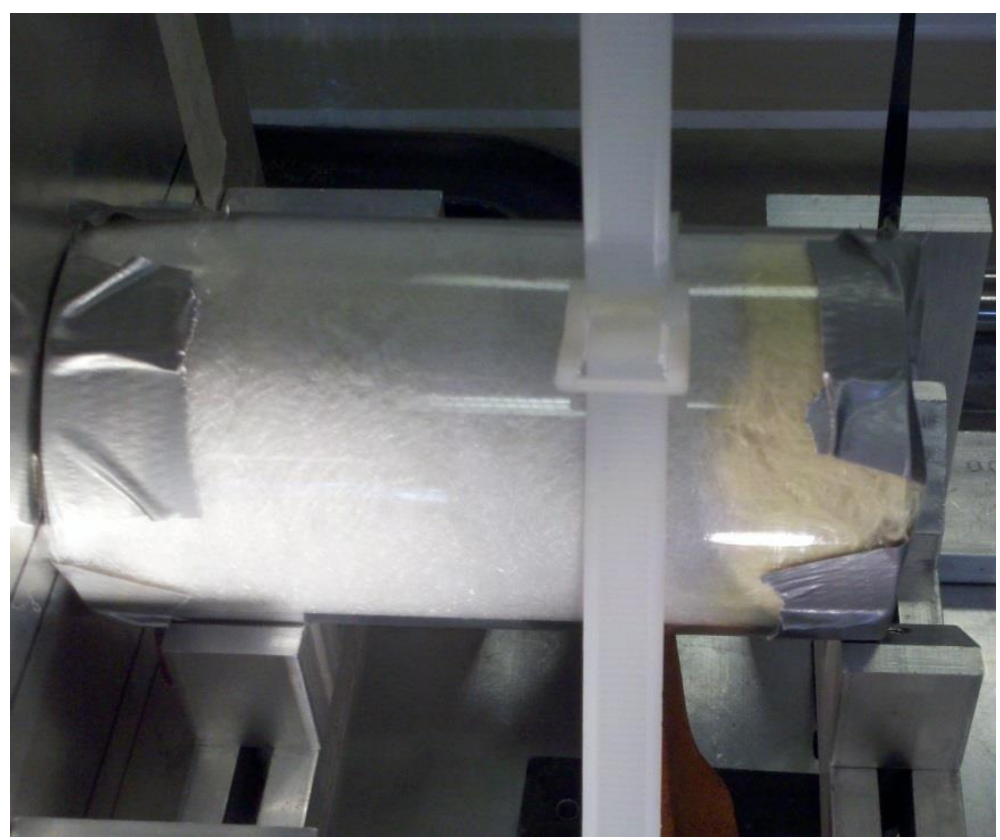

Figure 5.3 Kevlar-PET layered fiberfill secured in test fixture

\subsection{Results}

Results of each ballistic penetration test were fit to the Lambert-Jonas equation [66]:

$$
\mathrm{V}_{\text {out }}=\left(\mathrm{V}_{\text {in }}^{\mathrm{n}}-\mathrm{V}_{50}^{\mathrm{n}}\right)^{\frac{1}{\mathrm{n}}}
$$


with data falling below the $\mathrm{V}_{50}$ limit ignored. The results of this fitting are shown in Table 5.1. The exponent $\mathrm{n}$ takes on the value of 2 if a constant amount of kinetic energy is absorbed by the armor. Values of $\mathrm{n}$ higher than 2 indicate a reduction in the amount of kinetic energy absorbed as projectile velocities increase, and values less than 2 indicate an increase in energy absorbed with increasing velocity. Within the range tested, steel and PET show an increase in kinetic energy absorption with higher projectile velocities. This may be due to intrinsic rate dependence in the materials or in the projectile itself. Fiberfill, generally had an exponent of around 2, but varied between 1.804 and 2.453. This variation may be due to variable material properties, or may simply be an artifact of the error introduced by the $V_{50}$ calculation. Nonetheless, fiberfill compares favorably to both 1095 steel and PET sheets of similar area densities in terms of ballistic limit (Figure 5.4). The gas gun as configured for these experiments was unable to achieve velocities capable of defeating the Spectra or Kevlar fiberfill samples, showing that with an increase in fiber performance a marked increase in armor performance can potentially be realized.

The medium, and high-density Fiberloft samples are compared in Figure 5.5. Interestingly, the medium density samples appear to have the same, or perhaps slightly improved, performance versus the high density samples. This may indicate that the optimum tradeoff between fiber mobility and areal density occurs at a packing density less than $10.4 \mathrm{~kg} / \mathrm{m}^{2}$. The results for the low-density Fiberloft sample are shown in Figure 5.6. At around $300 \mathrm{~m} / \mathrm{s}$ an abrupt decrease in energy absorption is seen. This indicates a change in the mechanisms dissipating the projectile's kinetic energy, and may be the limit velocity at which the stick-slip mechanism is able to act on the projectile efficiently for this density of Fiberloft. 
Table 5.1 Ballistic penetration tests

\begin{tabular}{|c|c|c|c|c|c|c|}
\hline Test ID & Material & $\begin{array}{c}\text { Tube } \\
\text { Diameter } \\
{[\mathbf{m m}]}\end{array}$ & $\begin{array}{c}\text { Areal Density } \\
{\left[\mathbf{k g} / \boldsymbol{m}^{2}\right]}\end{array}$ & Projectile & $\mathbf{V}_{\mathbf{5 0}}[\mathbf{m} / \mathbf{s}]$ & $\mathbf{n}$ \\
\hline 001 & PET & - & 9.53 & BB & 238 & 1.468 \\
\hline 002 & 1095 Steel & - & 10.23 & BB & 282 & 1.567 \\
\hline 003 & Fiberloft & 47.6 & 10.67 & BB & 333 & 2.453 \\
\hline 004 & Fiberloft & 95.3 & 10.39 & BB & 336 & 1.919 \\
\hline 005 & Fiberloft & 197 & 10.38 & BB & 364 & 2.423 \\
\hline 006 & Polyfil & 47.6 & 10.67 & BB & 294 & 1.882 \\
\hline 007 & Kevlar 49 & 47.6 & 10.67 & BB & - & - \\
\hline 008 & Spectra 1000 & 47.6 & 10.67 & BB & - & - \\
\hline 009 & Fiberloft & 73 & 8.83 & BB & 356 & 2.251 \\
\hline 010 & Fiberloft & 73 & 4.42 & BB & 120 & - \\
\hline 011 & Fiberloft & 73 & 10.39 & FSP & 352 & 2.019 \\
\hline 012 & Fiberloft & 197 & 10.39 & FSP & 345 & 1.804 \\
\hline
\end{tabular}

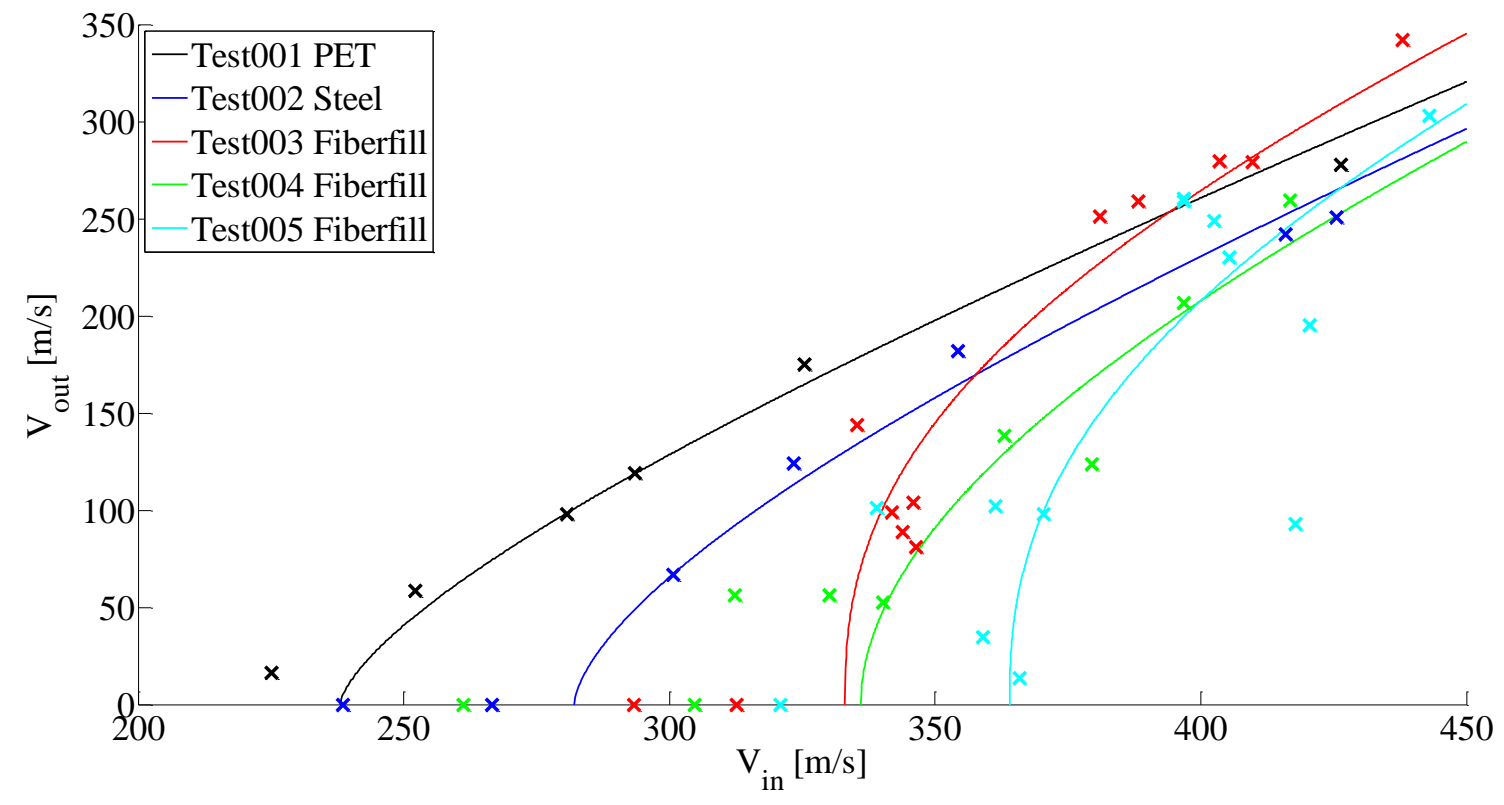

Figure 5.4 BB Tests; comparison of PET, Steel, and Fiberfill 


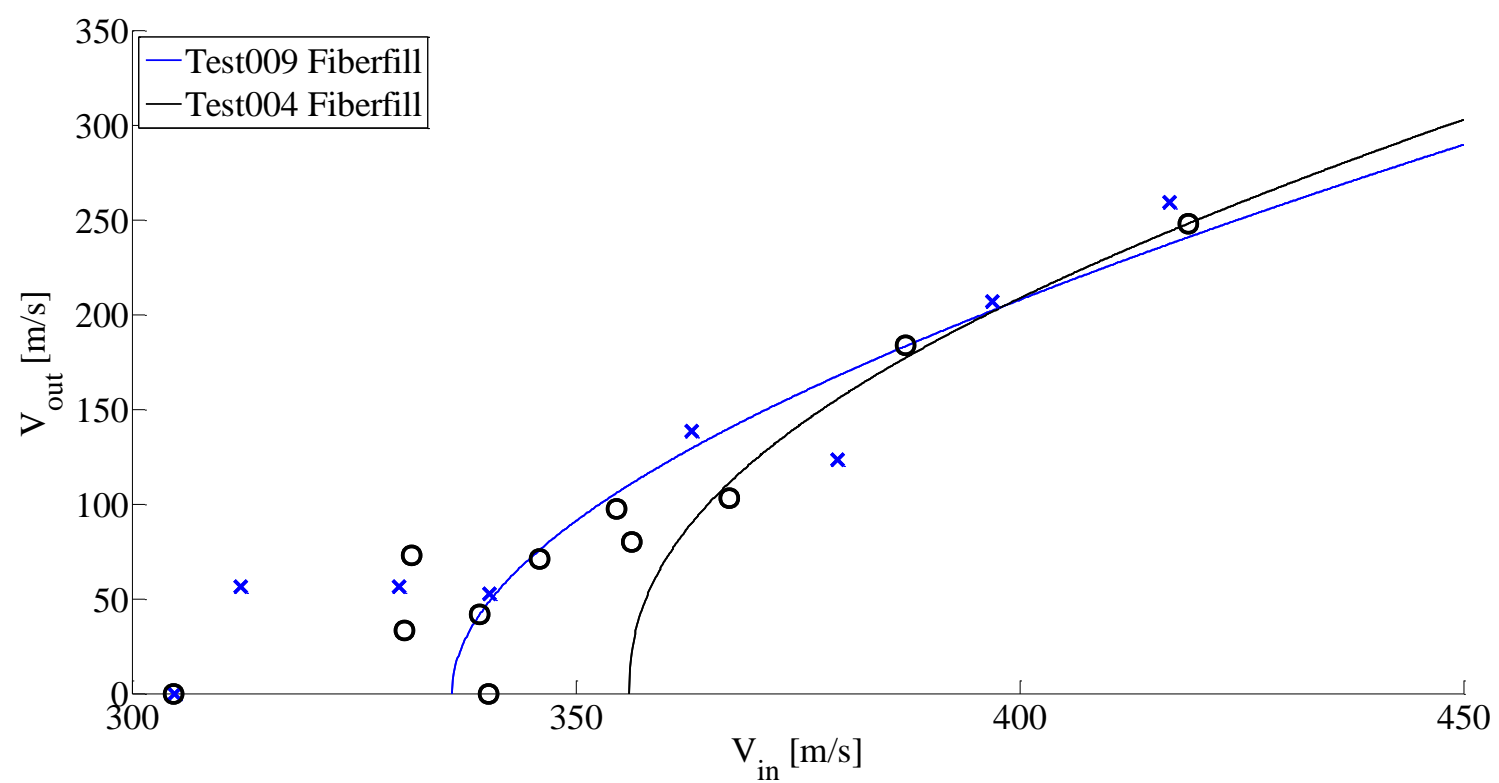

Figure 5.5 Comparison of Test ID $004\left(10.39\left[\mathrm{~kg} / \mathrm{m}^{2}\right]\right)$ and Test ID $009\left(8.83\left[\mathrm{~kg} / \mathrm{m}^{2}\right]\right)$

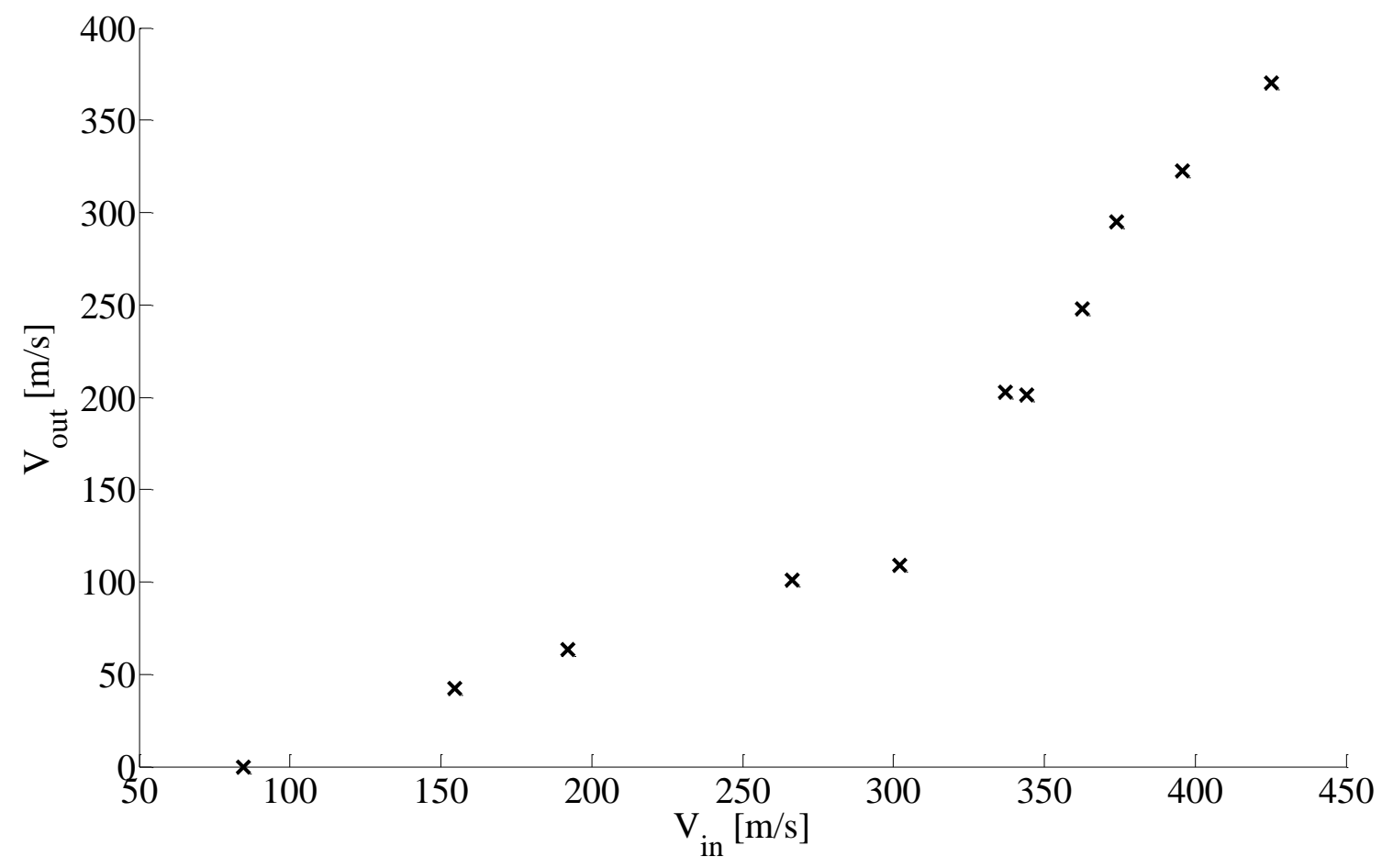

Figure 5.6 Test ID 010; low density Fiberloft vs BB projectile

Fragment simulating projectile test results are shown in Figure 5.7. The exit velocities near the ballistic limit were very difficult to determine in these tests because of the large amount of 
projectile tumbling. This tumbling tended to cause the higher velocity projectiles to veer off course, entirely miss the witness tape, and instead make contact with the steel plate surrounding the catch can. For exit velocities in excess of $200 \mathrm{~m} / \mathrm{s}$ the projectiles tended to be more stable, indicating a velocity limitation on the destabilization phenomena for the FSPs used. The tumbling projectiles interacted with increased volumes of the fiberfill material, which contributed to improved ballistic performance of the fiberfill when a projectile became unstable. Such a mechanism might prove useful in versus projectiles designed to tumble upon penetration of soft targets.

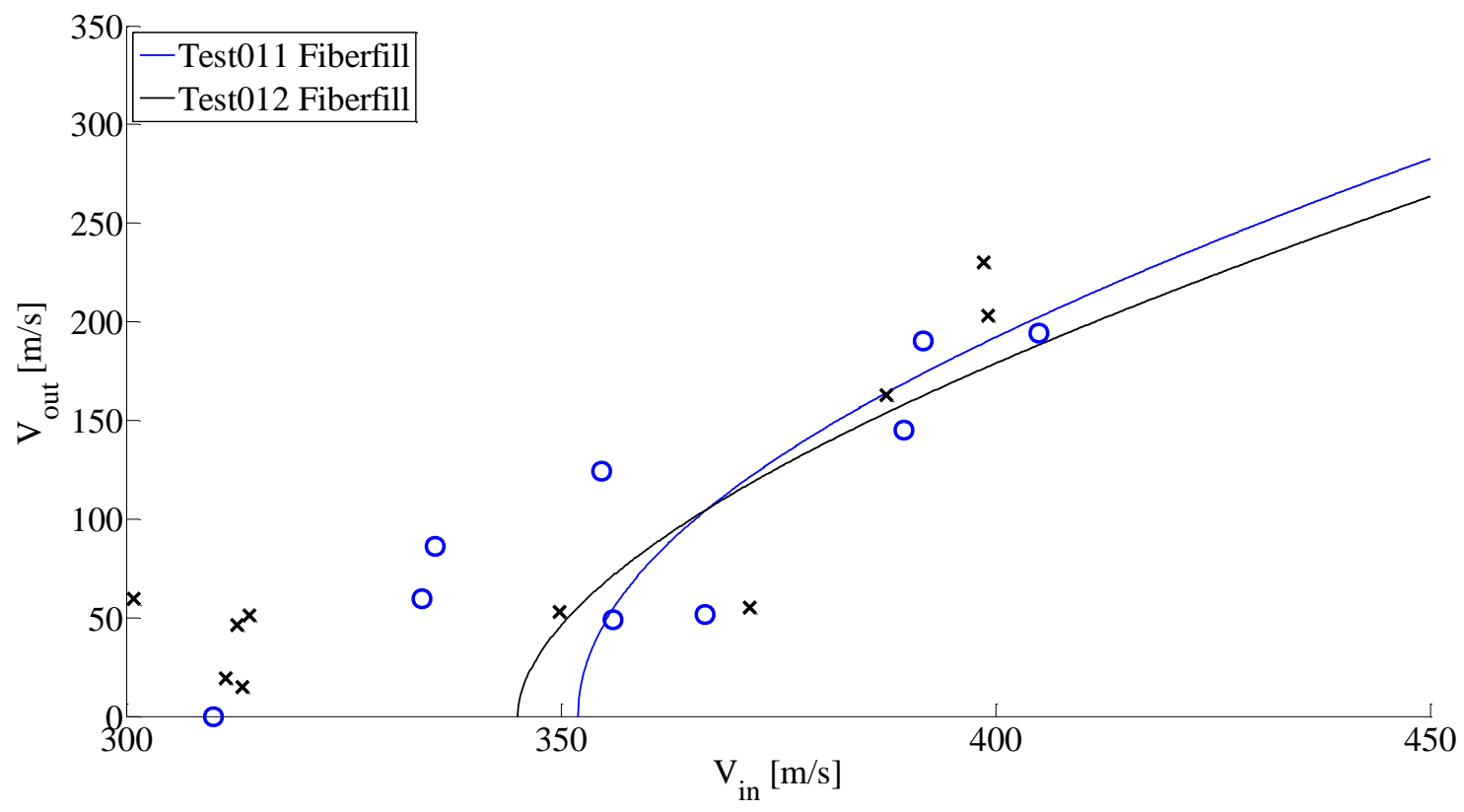

Figure 5.7 Test ID 011 and Test ID 012; Fiberloft vs FSPs

Images of the characteristic behaviors seen for penetrating projectiles versus Fiberloft and PET sheets are shown in Figure 5.8 through Figure 5.11. For the high velocity projectiles, a reduction in the amount of Fiberloft participating in the defeat is clearly evident from the exit photographs. The fibers that are involved in the defeat are seen to rotate, frictionally interacting with neighboring fibers, and align in the direction of the projectiles motion, forming long tails that slow the projectile even after it has left the sample's constraint tube (Figure 5.8 and Figure 5.9). 
This is in stark contrast to the monolithic PET sheets that were seen to behave in a very brittle manner at ballistic strain rates. Even in the lower velocity shots, PET quickly cracked and comminuted, absorbing no energy after the projectile had passed (Figure 5.11 and Figure 5.12).

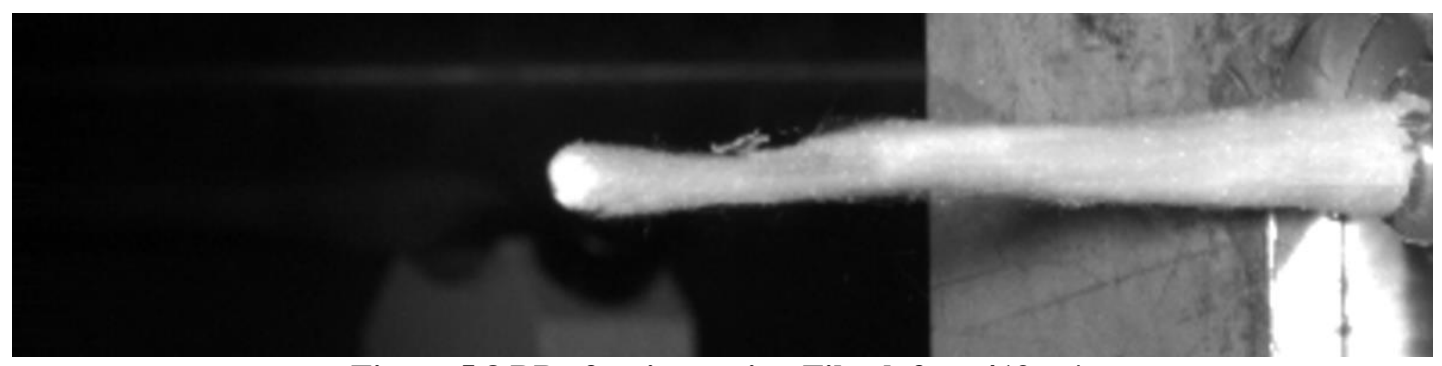

Figure 5.8 BB after impacting Fiberloft at $419 \mathrm{~m} / \mathrm{s}$

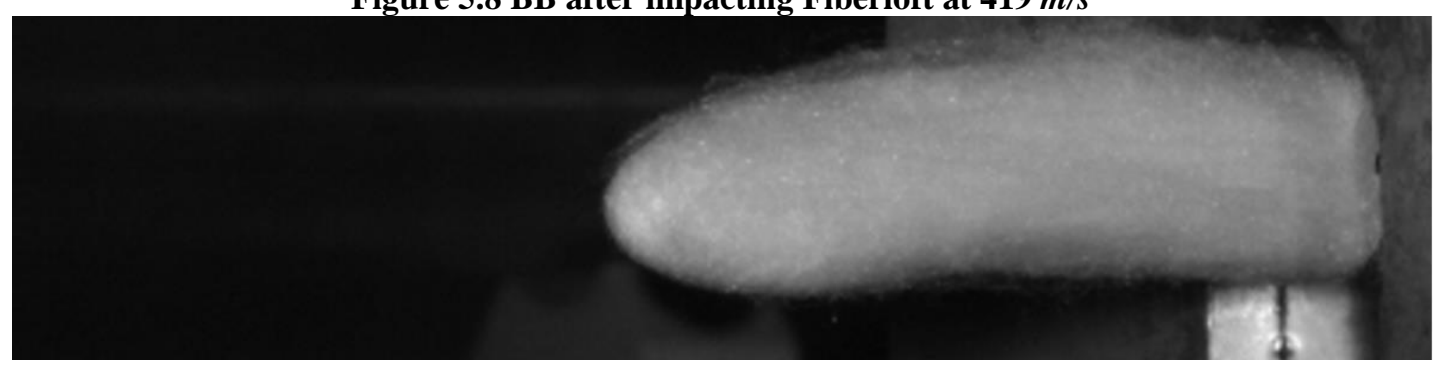

Figure 5.9 BB after impacting Fiberloft at $346 \mathrm{~m} / \mathrm{s}$

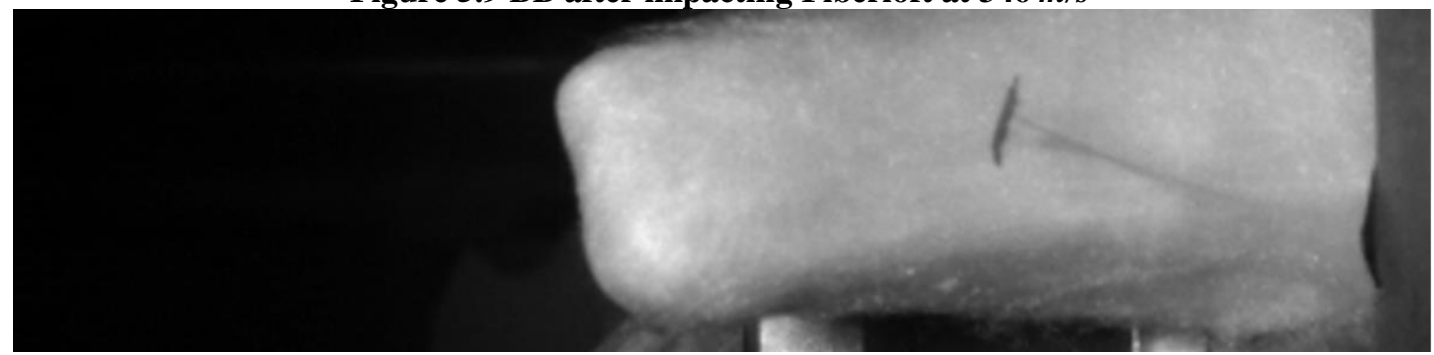

Figure 5.10 FSP tumbling after impacting Fiberloft at $367 \mathrm{~m} / \mathrm{s}$

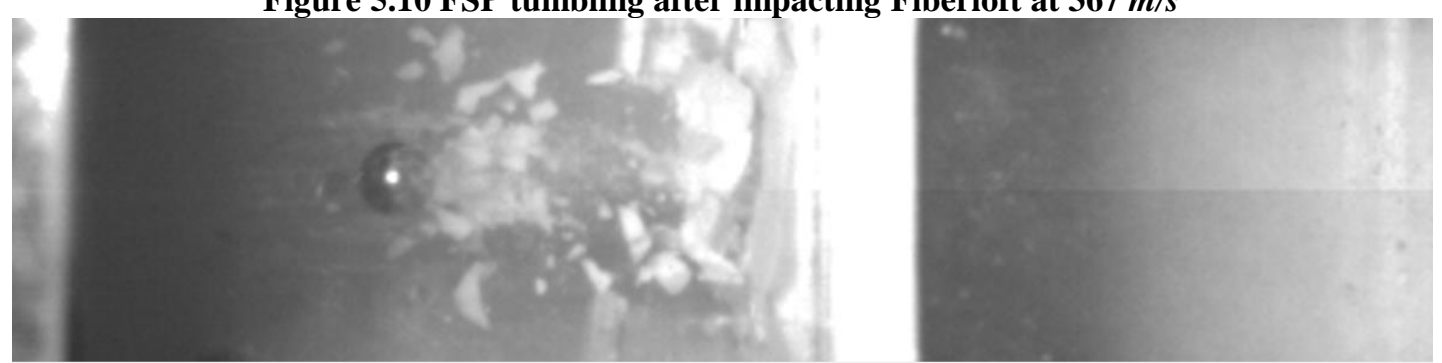

Figure 5.11 BB after impacting a PET sheet at $426 \mathrm{~m} / \mathrm{s}$ 


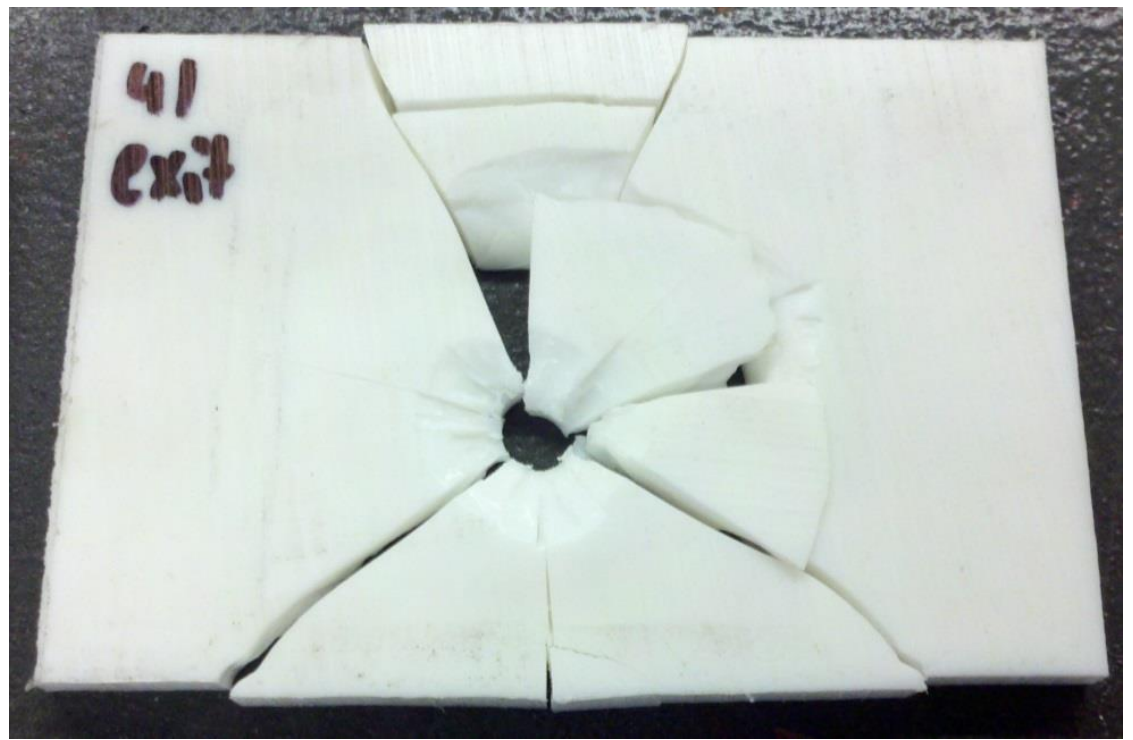

Figure 5.12 PET specimen showing cone and radial cracking after being impacted at $293 \mathrm{~m} / \mathrm{s}$

The ballistic limit could not be determined for the multi-layer Kevlar-PET fiberfill. Some interesting results regarding the deformation behavior of fiberfill during ballistic impact, however, were obtained from these tests. The projectile forces the top layer of Kevlar to fold in on itself, behaving like a net that envelops the projectile Figure 5.13. This entire mass of Kevlar is then dragged through the PET fiberfill behind it, forming a tail similar to those observed in 3.2. If the projectile has sufficient velocity it pierces the tape on the back of the sample, dragging a long tail of Kevlar behind it (Figure 5.14). For the range of velocities tested, projectiles were unable to break free from this tail and make contact with the witness tape. 


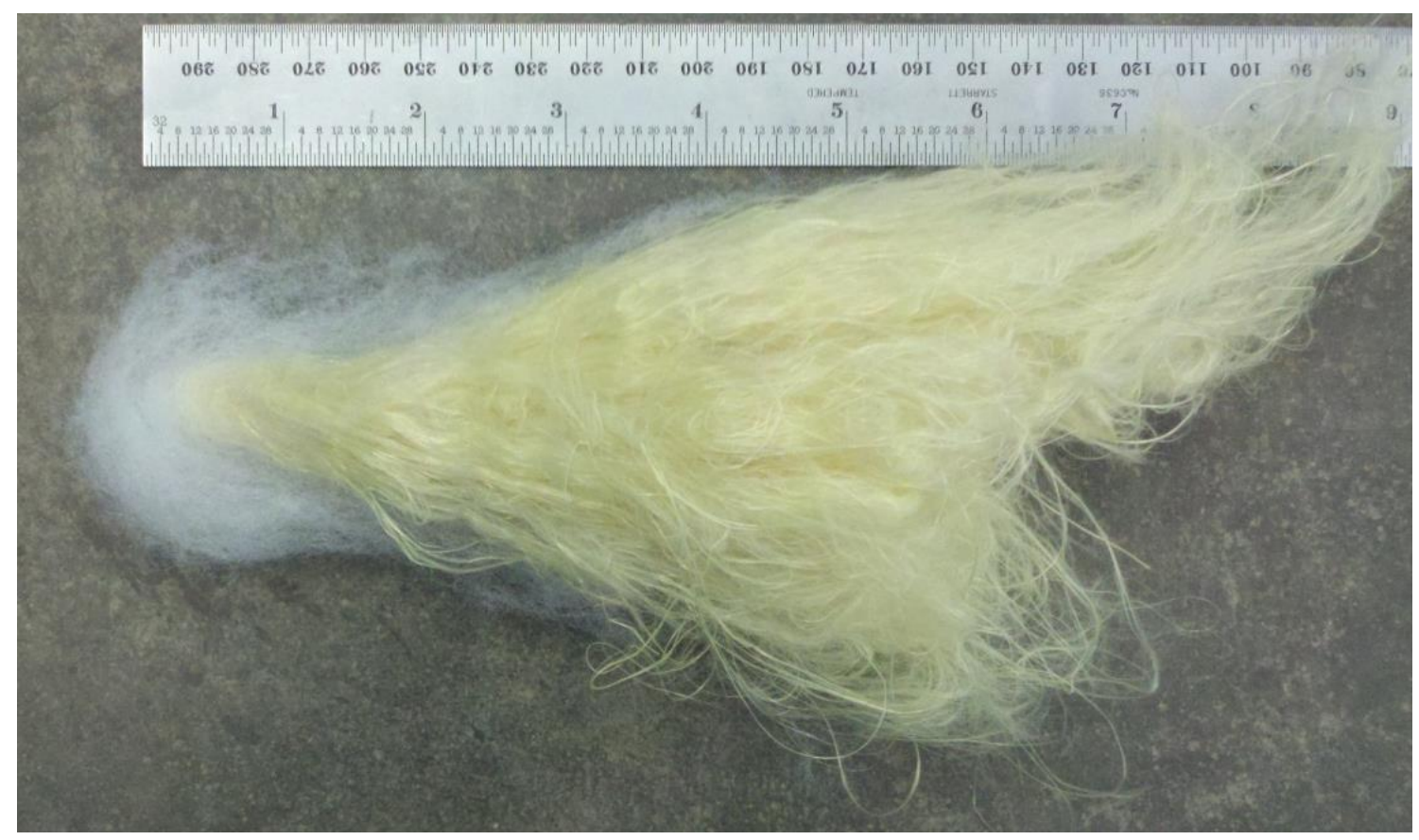

Figure 5.13 Kevlar/PET fiberfill enveloping a defeated projectile

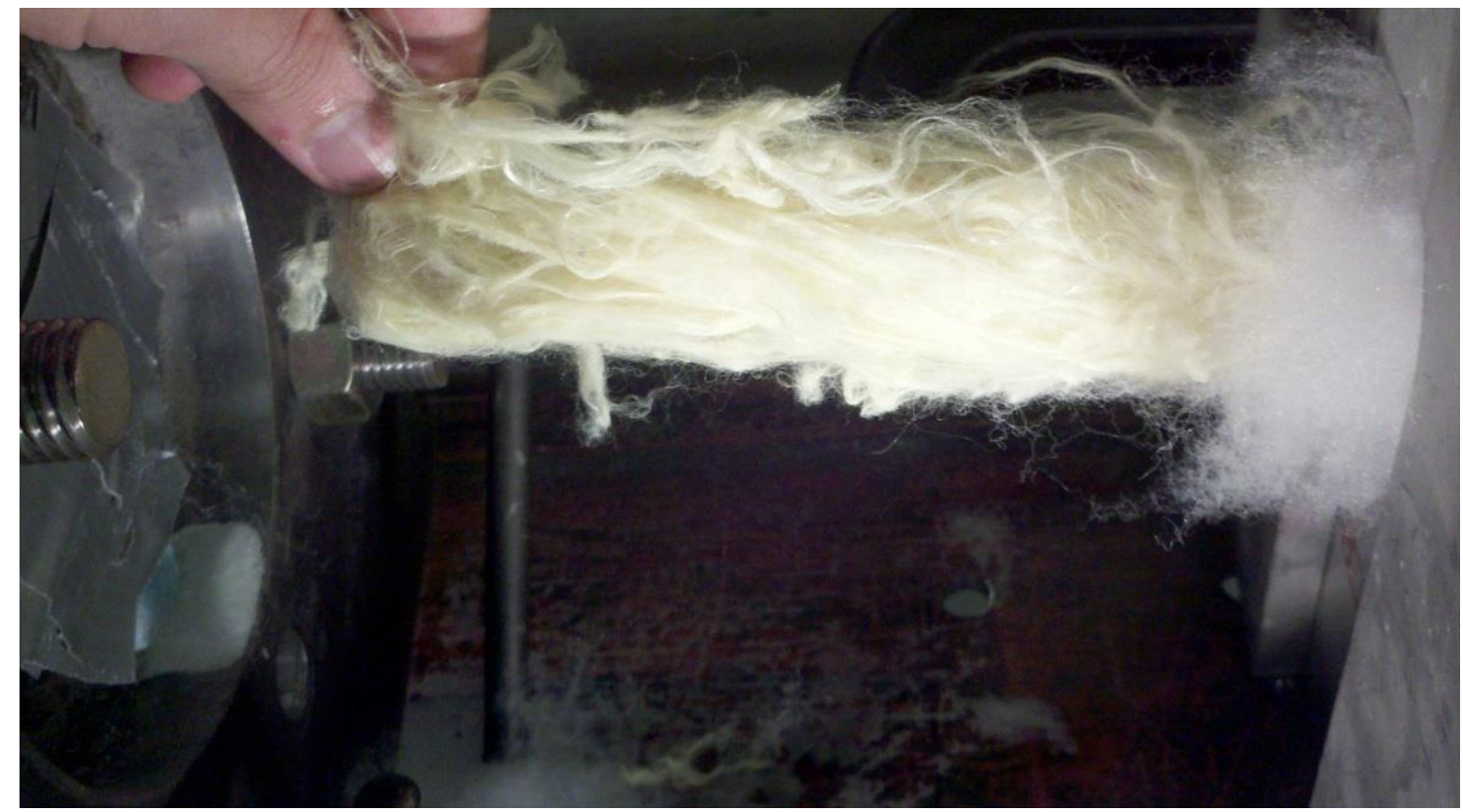

Figure 5.14 Projectile stopped in a Kevlar tail 


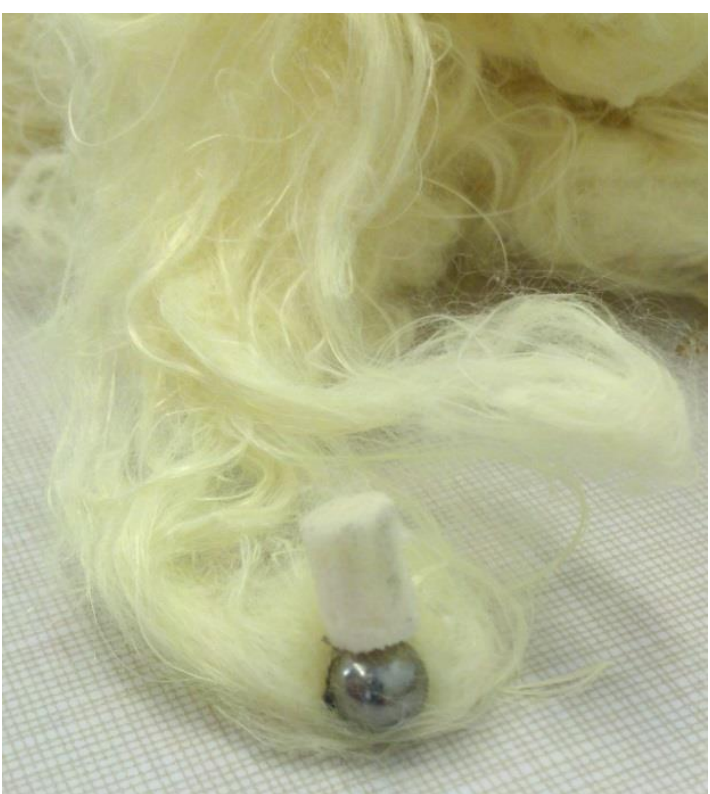

Figure 5.15 Defeated projectile with foam obturator still intact

Slug test results are shown in Figure 5.16. The data for these graphs was extracted from the high speed videos by tracking the back edge of the aluminum slug as it compressed the sample. Data for strains below $\sim 30 \%$ have been omitted due to a large degree of noise, and has instead been approximated by straight line from the origin. The remaining data was smoothed using a robust Lowess linear method in the Matlab curve fitting toolbox. The data is again truncated in the compaction regime due to a lack of certainty that the slug itself or the test fixture was not deforming under the applied stress. The results confirm the rate hardening trend seen in the drop tests, with the increased hardening likely coming from the entrapped air in the sample and constraint tube. 


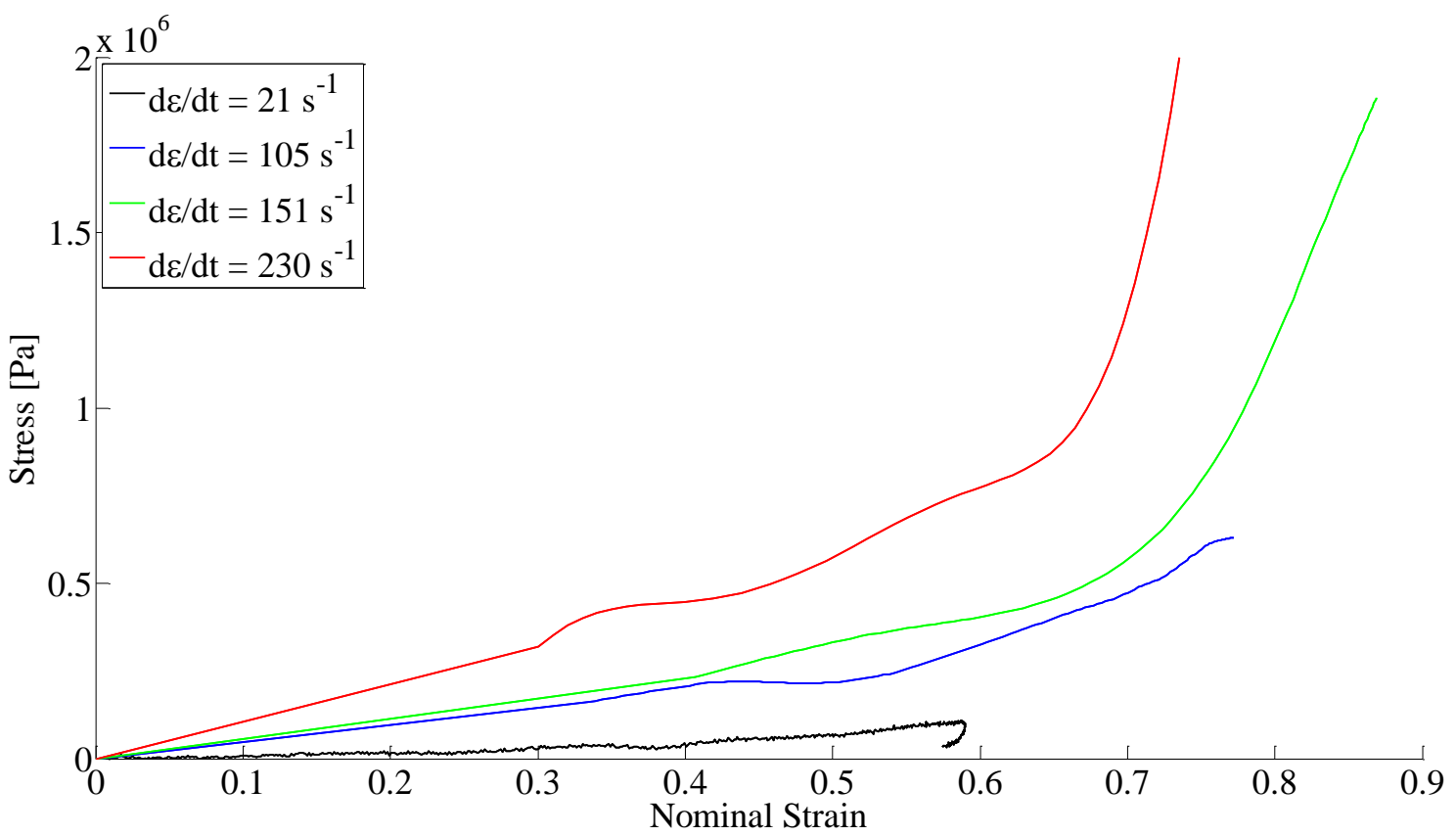

Figure 5.16 Slug tests; Stress-strain curves 


\section{Numerical Analysis}

Running a full set of ballistic experiments to understand the change in $\mathrm{V}_{50}$ with the variation of any one parameter is a time consuming process, often taking days of experimental work and data extraction before good results are obtained. Furthermore, in these experiments there are physical and practical limitations with what can be tested, and how these tests can be instrumented. The ability to setup and run a set of numerical simulations of the experiments prior to investing the time and capital to run physical tests would prove to be a useful tool in speeding up both research and design in the field of large displacement ballistic armor. To this end, various attempts at developing such a model were made throughout the course of working on this thesis. Initially, a simple open-celled foam model (MAT-57) was utilized in LS-DYNA to model the drop test experiments [67], and good results were obtained for low to moderate strain rates. This model was then extended using a simple damage criteria to simulate the gas gun BB experiments (Figure 6.1). The simple model failed to appropriately capture the physics occurring in the experiment, its weakness lying in this model's inability to accurately depict modes of deformation other than compression. Clearly, a more accurate constitutive model, with the ability to account for fiber orientation, arbitrary loading, and damage was needed. 


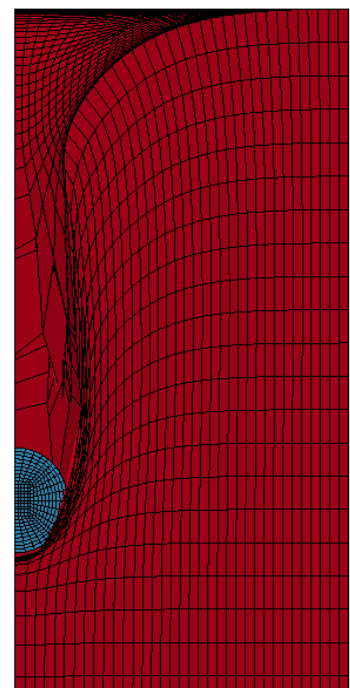

Figure 6.1 LS-DYNA MAT-57 ballistic simulation

\subsection{Models Considered}

Two models were found in the literature that had the potential to accurately capture the deformation behavior of entangled fiber materials under arbitrary loading conditions. These two models are the Planas model (P model) [68], [69], and the Jearanaisilawong model (JS model) [70]. Both models rely on the concepts of an effective fiber stretch [61], and an orientation distribution weighted response. Both models also rigorously account for the large rotations and extensions of the constituent fibers in the continuum, which will be necessary for simulation of the finite strains seen during ballistic experiments. The Cauchy stress of the incompressible P model is:

$$
\underline{\boldsymbol{\sigma}}=\int \hat{\mathrm{s}}_{\mathrm{f}}\left(\lambda_{\mathrm{f}}\right) \frac{[\underline{\mathbf{F}} \mathbf{N} \otimes \underline{\mathbf{F}} \mathbf{N}]}{\|\underline{\mathbf{F}} \mathbf{N}\|} \psi(\mathbf{N}, \mathbf{X}) \mathrm{d} \Omega-p \underline{\mathbf{I}}
$$

Where $\underline{\mathbf{F}}$ is the deformation gradient tensor, defined as: $\frac{\partial \mathbf{x}_{\mathbf{i}}}{\partial \mathbf{x}_{\mathrm{A}}}$ which maps material points in the reference configuration to material points in the present configuration,

$$
\mathbf{N}=\cos (\Theta) \mathbf{e}_{\mathbf{1}}+\sin (\Theta) \cos (\Phi) \mathbf{e}_{\mathbf{2}}+\sin (\Theta) \sin (\Phi) \mathbf{e}_{\mathbf{3}}
$$


is a unit vector in polar coordinates (Figure 6.2), $\mathrm{s}_{\mathrm{f}}$ is the user determined fiber stress-stretch constitutive relationship, $\psi$ is the orientation distribution function (ODF) of fibers in the continuum, $\lambda_{\mathrm{f}}$ is the fiber stretch, $\mathrm{p}$ is an arbitrary hydrostatic pressure, and $\underline{\mathbf{I}}$ is the unit tensor:

$$
\underline{\mathbf{I}}=\left[\begin{array}{lll}
1 & 0 & 0 \\
0 & 1 & 0 \\
0 & 0 & 1
\end{array}\right]
$$

The integral shown is taken on the upper spherical half space at each material point considered. This polar integration, which accomplishes the homogenization of an arbitrary fiber assemblage, weights the fiber stretch according to the orientation distribution function. Taking this spherical integral at each material point, for each increment during a finite element solution is, however, computationally costly. The JS model avoids this computational cost by using a tensorial measure of fabric anisotropy, thus losing the generality of an ODF-weighted integration, but consequently reducing computational cost.

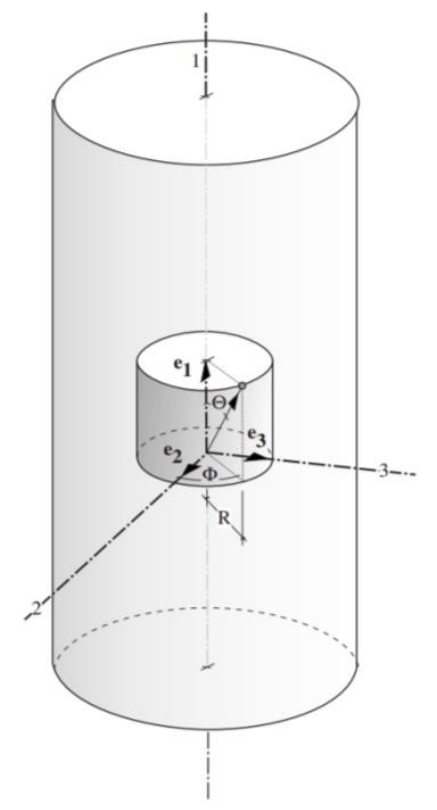

Figure 6.2 Coordinate system for the P model [68] 


\subsection{Fiberfill Continuum Model}

The JS model is, at its core, a homogenization of a multi-chain hyperelastic model. The strain energy function is derived from the force stretch relationship, the interfiber repulsion, and the resistance to bulk compaction. The model assumes a Langevin statistics based force-stretch relationship [60], [61], [71], [72], which phenomenologically captures uncrimping of the fibers. The framework of the model does not limit the selection of a force stretch relationship to one based on Langevin statistics, but it will be the fiber constitutive relationship adopted in this thesis. The JS model utilizes a tensorial representation of the fiber orientation distribution and the distribution of junction-to-junction distances, thus eliminating the need to perform polar integration at each material point. This comes at the cost of losing the ability to represent arbitrary fiber distributions, as in the P model [73]. The structural tensor that captures orientation effects is referred to as the "fabric ellipsoid" or "texture" tensor, $\underline{\mathbf{G}}_{\mathbf{0}}$. A derivation for the elastic and inelastic parts of the JS model is included in the appendix. The Cauchy stress is written as follows:

$$
\underline{\boldsymbol{\sigma}}=\frac{\mu}{\mathrm{J} \operatorname{tr}\left(\underline{\mathbf{G}}_{0}\right)}\left(\frac{\lambda_{1}}{\lambda_{\mathrm{f}}} \beta\{\chi\} \underline{\mathbf{F}} \underline{\mathbf{G}}_{0} \underline{\mathbf{F}}^{\mathrm{T}}-\lambda_{1} \beta_{0} \underline{\mathbf{R}}_{\underline{\mathbf{G}}_{0}} \underline{\mathbf{R}}^{\mathrm{T}}\right)-\kappa\left(\mathrm{J}^{-\mathrm{n}}-1\right) \underline{\mathbf{I}}
$$

For inelastic deformation the texture tensor $\underline{\mathbf{G}}_{0}$ is replaced by the evolving structural tensor $\underline{\mathbf{G}}_{\mathrm{t}}$.

The stress in the inelastic JS model depends on the elastic portion of the deformation and the inelastic evolution of the texture tensor. The Cauchy stress is calculated by:

$$
\underline{\boldsymbol{\sigma}}=\frac{\mu}{\mathrm{J} \operatorname{tr}\left(\underline{\mathbf{G}}_{\mathrm{t}}\right)}\left(\frac{\lambda_{1}}{\lambda_{\mathrm{f}}^{\mathrm{e}}} \beta\left\{\chi^{\mathrm{e}}\right\} \underline{\mathbf{F}}^{\mathrm{e}} \underline{\mathbf{G}}_{\mathrm{t}} \underline{\mathbf{F}}^{\mathrm{e}^{\mathrm{T}}}-\lambda_{1} \beta_{0} \underline{\mathbf{R}}^{\mathrm{e}} \underline{\mathbf{G}}_{\mathrm{t}} \underline{\mathbf{R}}^{\mathrm{e}^{\mathrm{T}}}\right)-\kappa\left(\mathrm{J}^{\mathrm{e}-\mathrm{n}}-1\right) \underline{\mathbf{I}}
$$

The model implemented in this work deviates from the original JS model in several key ways. First, the constitutive relationship is augmented by a bulk compression term of the power law form suggested by van-Wyk. Second, the inelastic rotation is assumed to be: $\underline{\mathbf{R}}^{\mathrm{i}}=\underline{\mathbf{I}}$. Therefore 
only "elastic" rotations occur. This forces the solution of the inelastic component of the deformation to be unique [74], and leads to the same result for the inelastic spin as assumed in the JS inelastic model:

$$
\underline{\mathbf{W}}^{\mathrm{i}}=\underline{\mathbf{0}}
$$

Eq 6.6

and is helpful in casting the constitutive relationship in a corotational framework, as discussed in 6.2.2. Third, the model is augmented by a progressive damage evolution law that models the effects of fiber slip and disentanglement.

\subsubsection{Progressive Damage Model}

The JS model fails to capture the effects of fibers slipping from junction points [75]. This leads to greatly over predicted stresses at high strains, as real fiberfill displays significant softening after reaching a peak stress due to the effects of accumulated damage. In order to capture these effects the principles of continuum damage mechanics (CDM) were applied, within the framework of the JS model, to formulate a simple damage evolution law.

Kachanov was the first to introduce the concept of using a continuous variable to capture the microscopic effects of damage accumulation [76]. Damage models based on CDM utilize this continuous, and monotonically increasing damage variable, $\mathrm{d}$, to track the effects of accumulating damage in the continuum. The stress in a damaged body is related to the effective stress by the relation:

$$
\underline{\tilde{\boldsymbol{\sigma}}}=\frac{\underline{\boldsymbol{\sigma}}}{1-\mathrm{d}}
$$

Here, the quantity (1-d) can be interpreted as the effective area on which the stress is acting (Figure 6.3), $\underline{\tilde{\boldsymbol{\sigma}}}$ is the effective stress acting on the effective area, and $\underline{\boldsymbol{\sigma}}$ is the stress acting on the reference area [77]. This concept in the present model amounts to a scaling back of the effective strain energy due to the elongation of fibers. This is expressed as: 


$$
\underline{\boldsymbol{\sigma}}=(1-\mathrm{d})\left(\frac{\mu}{\mathrm{J} \operatorname{tr}\left(\underline{\mathbf{G}}_{\mathrm{t}}\right)}\left(\frac{\lambda_{1}}{\lambda_{\mathrm{f}}^{\mathrm{e}}} \beta\left\{\chi^{\mathrm{e}}\right\} \underline{\mathbf{F}}^{\mathrm{e}} \underline{\mathbf{G}}_{\mathrm{t}} \underline{\mathbf{F}}^{\mathrm{e}^{\mathrm{T}}}-\lambda_{\mathrm{l}} \beta_{0} \underline{\mathbf{R}}^{\mathrm{e}} \underline{\mathbf{G}}_{\mathrm{t}} \underline{\mathbf{R}}^{\mathrm{e}^{\mathrm{T}}}\right)-\kappa\left(\mathrm{J}^{\mathrm{e}-\mathrm{n}}-1\right) \underline{\mathbf{I}}\right)
$$

A simple phenomenological model based on a Weibull analysis of fiber pullout is discussed in Krajcinovic [78]. The form of this model is adopted in the present analysis. The proposed model for the progressive growth of damage in fiberfill material is:

$$
\begin{gathered}
\mathrm{d}_{\mathrm{t}+\mathrm{dt}}=\left\{\begin{array}{c}
1-\exp \left(-\left(\mathrm{A}\left(\frac{\sigma_{\mathrm{eff}}}{\sigma_{0}}-1\right)\right)^{\mathrm{m}_{\mathrm{d}}}\right) \\
\mathrm{d}_{\mathrm{t}} ; \sigma_{\mathrm{eff}} \leq \sigma_{0}
\end{array}\right) \sigma_{\mathrm{eff}}>\sigma_{0} \\
\mathrm{~A}=\frac{2 \mathrm{~L}_{\mathrm{ch}} \sigma_{0}^{2}}{2 \mu \beta\left(\frac{\lambda_{\mathrm{f}}^{\mathrm{e}}}{\lambda_{1}}\right) G_{\text {frac }}-\mathrm{L}_{\mathrm{ch}} \sigma_{0}^{2}}
\end{gathered}
$$

Here, from the JS model, $\mu$ is the modulus parameter with units of pressure, $\beta(\cdot)$ is the inverse Langevin function, $\lambda_{\mathrm{f}}^{\mathrm{e}}$ is the elastic effective fiber stretch, and $\lambda_{1}$ is the locking stretch. $\sigma_{\text {eff }}$ is the Von Mises effective stress defined as:

$$
\sigma_{\text {eff }}=\frac{1}{1-\mathrm{d}} \sqrt{\frac{3}{2}(\underline{\boldsymbol{\sigma}}-\operatorname{tr}(\underline{\boldsymbol{\sigma}}) \underline{\mathbf{I}})^{2}}
$$

where $\operatorname{tr}(\cdot)$ is the trace operator, $\sigma_{0}$ is the effective stress at which damage accumulation begins to occur, $G_{\text {frac }}$ is a fit parameter with units of energy divided by area, $\mathrm{m}$ is a dimensionless fit parameter, and $\mathrm{L}_{\mathrm{ch}}$ is the element characteristic length. The effective Von Mises stress is selected as the driving variable in the damage model based on the observation that damage accumulates earlier in shear deformation than in tensile deformation. An equivalent formulation based on the effective deviatoric strain could alternatively be implemented. In order to capture complex anisotropic damage growth, growth laws for different modes would need to be implemented. An 
example of an anisotropic continuum damage model for composite materials can be found in Matzenmiller [79].

The element characteristic length is introduced to reduce the mesh dependency of the damage model. Damage accumulation suffers from non-physical localization effects and element size dependent energy dissipation in finite element modeling. These spurious effects are reduced by the introduction of the element characteristic length to the damage evolution law [80]. The form of the "fracture energy" variable, A, used in the present model is analogous to that used in Ridruejo [69], but with a variable, nonlinear modulus parameter that scales with the effective stretch ratio.

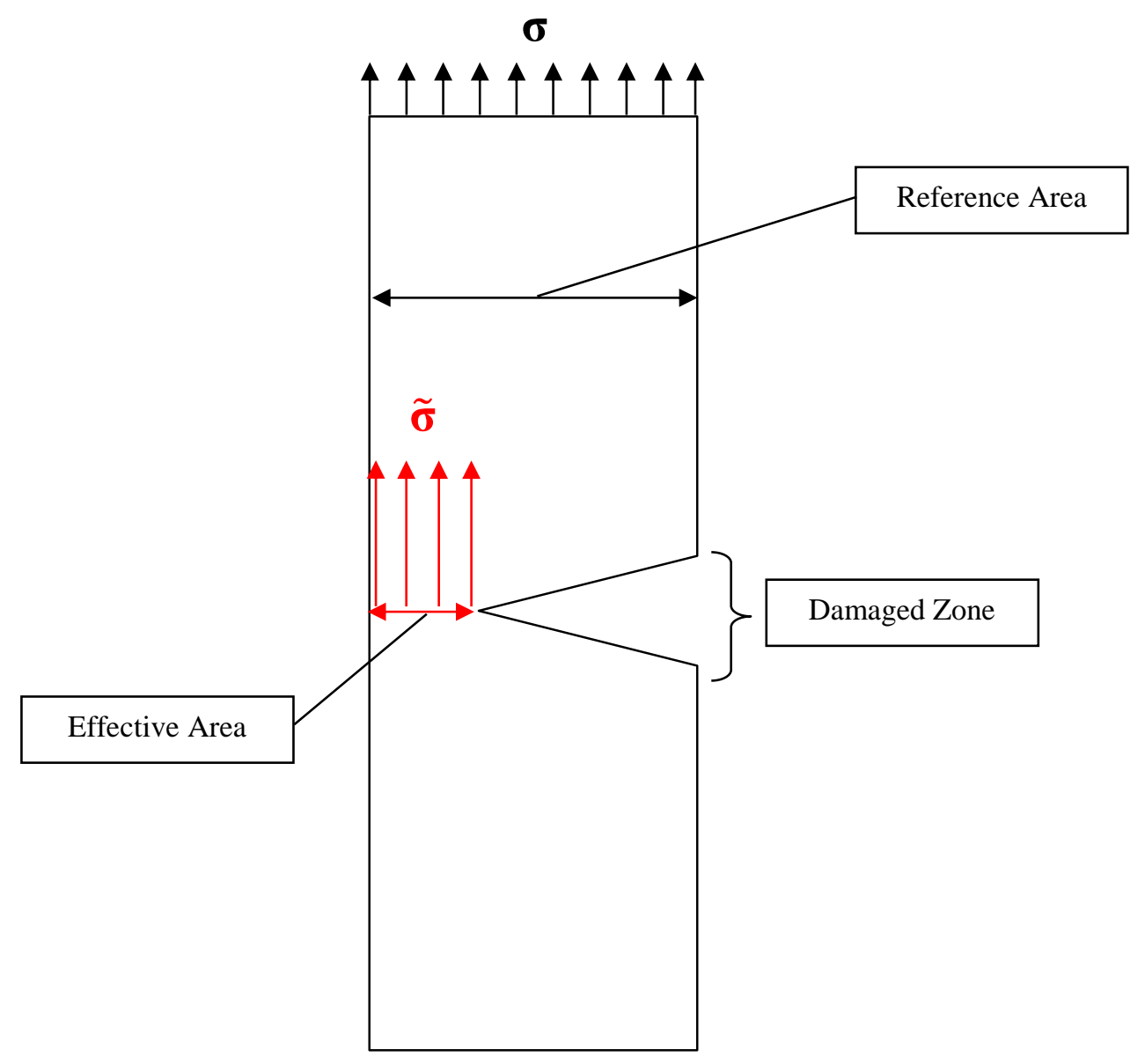

Figure 6.3 Illustration of the concept of effective area. 
This model is subject to the thermodynamic restriction that damage must be dissipative, and thus cannot decrease. Therefore:

$$
\mathrm{d}=\max [0, \max (\mathrm{d})]
$$

It is also restricted by the criteria:

$$
\mathrm{d}=[0,1]
$$

since damage cannot physically be greater than $100 \%$.

The damage model is completed by a viscous regularization of the damage growth based on the generalized Duvaut-Lions method [81]. This is expressed as:

$$
d_{t+\Delta t}=\frac{\Delta t}{\eta_{d}+\Delta t} d_{t+\Delta t}^{t r}+\frac{\eta_{d}}{\eta_{d}+\Delta t} d_{t}
$$

Here $\mathrm{d}^{\text {tr }}$ is the value calculated from the damage evolution law, and $\mathrm{d}$ is the viscous regularized damage variable, which approaches $d^{\text {tr }}$ as $t \rightarrow \infty$. Viscous regularization minimizes damage runaway in the event of localization and stabilizes the solution in the softening regime, but it also increases the amount of energy absorbed during damage accumulation, and adds a time dependent component to damage growth. These effects must be accounted for when calibrating the damage model and it is suggested that a very low value of the damage viscosity, $\eta_{d}$, be used.

\subsubsection{Abaqus implementation}

Modeling of short timescale events such as ballistic impact and blast, are well suited to an explicit dynamic finite element solution [67], [82]. The JS model, including the proposed additions, was implemented in Abaqus Explicit as a vectorized user material model (VUMAT).

The Abaqus VUMAT interface uses a corotational framework where the reference system rotates along with the material. The rotating material frame is rotated back to the global reference frame 
external to the VUMAT subroutine. Thus, when specifying the Cauchy stress within the VUMAT the corotational stress must be used, specified by the equation:

$$
\underline{\boldsymbol{\sigma}}^{\text {corotational }}=\underline{\mathbf{R}}^{\mathrm{T}} \underline{\boldsymbol{\sigma}} \underline{\mathbf{R}}
$$

This is accommodated by formulating the constitutive model using the stretch tensor $\underline{\mathbf{U}}$ in place of the deformation gradient tensor $\underline{\mathbf{F}}$. Where, from the polar decomposition of the deformation gradient:

$$
\underline{\mathbf{F}}=\underline{\mathbf{R}} \underline{\mathbf{U}}
$$

Eq 6.16

Some difficulty arises from the calculation of the elastic stretch from the inelastic stretch. Using the assumption mentioned previously, that $\underline{\mathbf{R}}^{\mathrm{i}}=\underline{\mathbf{I}}, \underline{\mathbf{R}}=\underline{\mathbf{R}}^{\mathrm{e}}$ the elastic stretch can be calculated as:

$$
\underline{\mathbf{U}}^{\mathrm{e}}=\underline{\mathbf{U}} \underline{\mathbf{U}}^{\mathrm{i}^{-1}}
$$

The damage affected Cauchy stress internal to the VUMAT is then expressed as:

$$
\underline{\boldsymbol{\sigma}}^{\text {corotational }}=(1-\mathrm{d})\left(\frac{\mu}{\mathrm{J} \operatorname{tr}\left(\underline{\mathbf{G}}_{\mathrm{t}}\right)}\left(\frac{\lambda_{1}}{\lambda_{\mathrm{f}}^{\mathrm{e}}} \beta\left\{\chi^{\mathrm{e}}\right\} \underline{\mathbf{U}}^{\mathrm{e}} \underline{\mathbf{G}}_{\mathrm{t}} \underline{\mathbf{U}}^{\mathrm{e}}-\lambda_{\mathrm{l}} \beta_{0} \underline{\mathbf{G}}_{\mathrm{t}}\right)-\kappa\left(\mathrm{J}^{\mathrm{e}-\mathrm{n}}-1\right) \underline{\mathbf{I}}\right)
$$

Where the elastic effective stretch is:

$$
\lambda_{\mathrm{f}}^{\mathrm{e}}=\sqrt{\frac{\operatorname{tr}\left(\underline{\mathbf{U}}^{\mathrm{e}} \underline{\mathbf{G}}_{\mathrm{t}} \underline{\mathbf{U}}^{\mathrm{e}^{\mathrm{T}}}\right)}{\operatorname{tr}\left(\underline{\mathbf{G}}_{\mathrm{t}}\right)}}
$$

The model is written so as to include all necessary subroutines without requiring additional software libraries, such as BLAS or LAPACK. An LU decomposition subroutine from [83] is used for calculating matrix inverses. A simple pseudorandom number generator is included if a stochastic component to the damage evolution, or any other material property, is desired. The 
user also has the option to delete elements based on the maximum allowable accumulated damage. This allows the model to capture the post-localization ripping and tearing effects seen in the experiments (Figure 4.13)

The inverse Langevin function, $\beta$, defined as:

$$
x=\operatorname{coth}(\beta(x))-\frac{1}{\beta(x)}
$$

is approximated by the inverse of a Taylor series expansion of, $\mathcal{L}$, the Langevin function:

$$
\mathrm{L}(\mathrm{x})=\operatorname{coth}(\mathrm{x})-\frac{1}{\mathrm{x}}
$$

Thus the inverse Langevin function is approximated as [84]:

$$
\beta(x)_{\text {approx }}=3 x+\frac{9}{5} x^{3}+\frac{297}{175} x^{5}+\frac{1539}{875} x^{7}+\frac{126117}{67375} x^{9}+\ldots
$$

A representation of this form does away with the inextensibility limit and has reduced accuracy in the neighborhood of $x=1$. Alternatively, a Padé approximant can be used, which has better fidelity near the inexstensible limit [85]:

$$
\beta(\mathrm{x})_{\text {Padé }}=3 \mathrm{x} \frac{35-12 \mathrm{x}^{2}}{35-33 \mathrm{x}^{2}}
$$

The Padé approximant gives a more accurate response than the Taylor series approximation with fewer terms, but suffers from an instability at $x=1$. Both functions are included in the VUMAT code.

The complete FORTRAN code is included in the appendix. 


\subsubsection{Model Results}

The model remains to be appropriately fit to a specific entangled fiber armor material. Some results for material parameters shown in Table 6.1 appear to be a reasonable approximation for the tension and compression response of Fiberloft. However, the structure is assumed to be initially isotropic for the results shown, which is known to be false based on the material characterization tests conducted in Chapter 4.

Table 6.1 Parameters used in model results (unless otherwise noted)

\begin{tabular}{|c|c|c|c|}
\hline Number & Parameter & Value & Units \\
\hline 1 & $\mu$ & 5000 & {$[P a]$} \\
\hline 2 & $\lambda_{1}$ & 2 & - \\
\hline 3 & $\kappa$ & 259 & {$[P a]$} \\
\hline 4 & $\mathrm{n}$ & 3.61 & - \\
\hline 5 & $\mathrm{G}_{0}(1,1)$ & 1 & - \\
\hline 6 & $\mathrm{G}_{0}(2,2)$ & 1 & - \\
\hline 7 & $\mathrm{G}_{0}(3,3)$ & 1 & - \\
\hline 8 & $\mathrm{G}_{0}(1,2)$ & 0 & - \\
\hline 9 & $\mathrm{G}_{0}(2,3)$ & 0 & - \\
\hline 10 & $\mathrm{G}_{0}(3,1)$ & 0 & - \\
\hline 11 & $\tau_{0}$ & 5 & {$[P a]$} \\
\hline 12 & $\alpha$ & 175 & {$[P a]$} \\
\hline 13 & $\mathrm{~m}$ & 1.5 & - \\
\hline 14 & $v$ & 0.25 & - \\
\hline 15 & $\mathrm{~S}_{0}$ & 10 & {$[P a]$} \\
\hline 16 & $\dot{\gamma}$ & 0.005 & {$[1 / s]$} \\
\hline 17 & $\mathrm{G}_{\text {frac }}$ & 3 & {$\left[\mathrm{~J} / \mathrm{m}^{2}\right]$} \\
\hline 18 & $\mathrm{~m}_{\mathrm{d}}$ & 1 & - \\
\hline 19 & $\sigma_{0}$ & 650 & {$[P a]$} \\
\hline 20 & $\eta_{\mathrm{d}}$ & 0 & {$[s]$} \\
\hline 21 & $\mathrm{~d}_{\max }$ & 1 & - \\
\hline & & & \\
\hline
\end{tabular}

Quasistatic simulations of the tension experiments were run using the JS model (Figure 6.4). Good results in the low stretch regime were obtained (Figure 6.5 and Figure 6.6). Adequate strain softening behavior could not be achieved with the proposed damage model without a very large 
increase in the damage viscosity parameter (Figure 6.7). However, an improvement in the model predictions is seen with the proposed progressive damage law in effect (Figure 6.8). With too low of a damage viscosity the solution tends to localize into a single plane of elements (Figure 6.9), unload, then load the next plane of elements until it too localizes. This leads to oscillations in the stress in the high strain regime, and is due to the ill posed nature of the problem at some point during the softening [86]. The localization seen here is a consequence of the discretization of the body into finite elements. Methods for circumventing non-physical localization instability include gradient smoothing, inter-element averaging, and non-local formulations. In order to obtain a physically realistic response in the softening regime, one of these schemes should be adopted. As it stands, a sufficient amount of damage viscosity can be added to approximate the solution desired. The elements should then be eroded to prevent large stress oscillations.

Results for the variation of $G_{\text {frac }}$ are shown in Figure 6.10. Increasing this parameter does not affect the localization issue, but it does increase the peak stress at failure and increases the energy absorbed up to fracture.

Uniaxial tensile simulations were also run on a single element model. In the single element, no spurious localization can occur, so it should give insight into the damage model's behavior if damage could be adequately spread to neighboring elements. Results are shown in Figure 6.11.

Results for constrained compression are shown in Figure 6.12. These results show good agreement with experimental results for fiberfill, but predict excessively high stiffness in the precompaction regime, perhaps owing to the fact that the model is not yet appropriately fit to fiberfill.

In general, the proposed material model appears to capture the relevant mechanisms in the deformation modes examined, but still requires some work in terms of complete fitting to the data and a mesh-insensitive formulation for progressive damage modeling. 


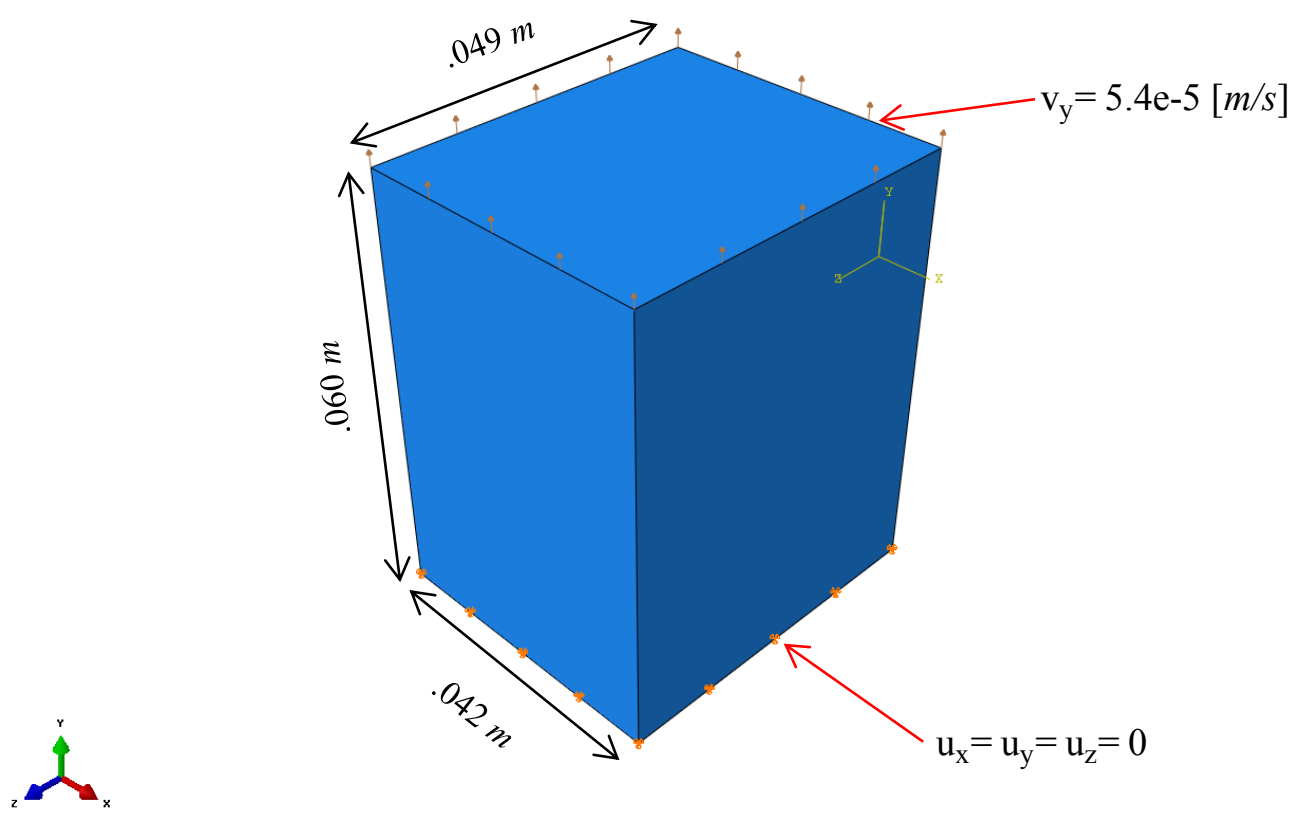

Figure 6.4 Boundary conditions and geometry for tension simulation

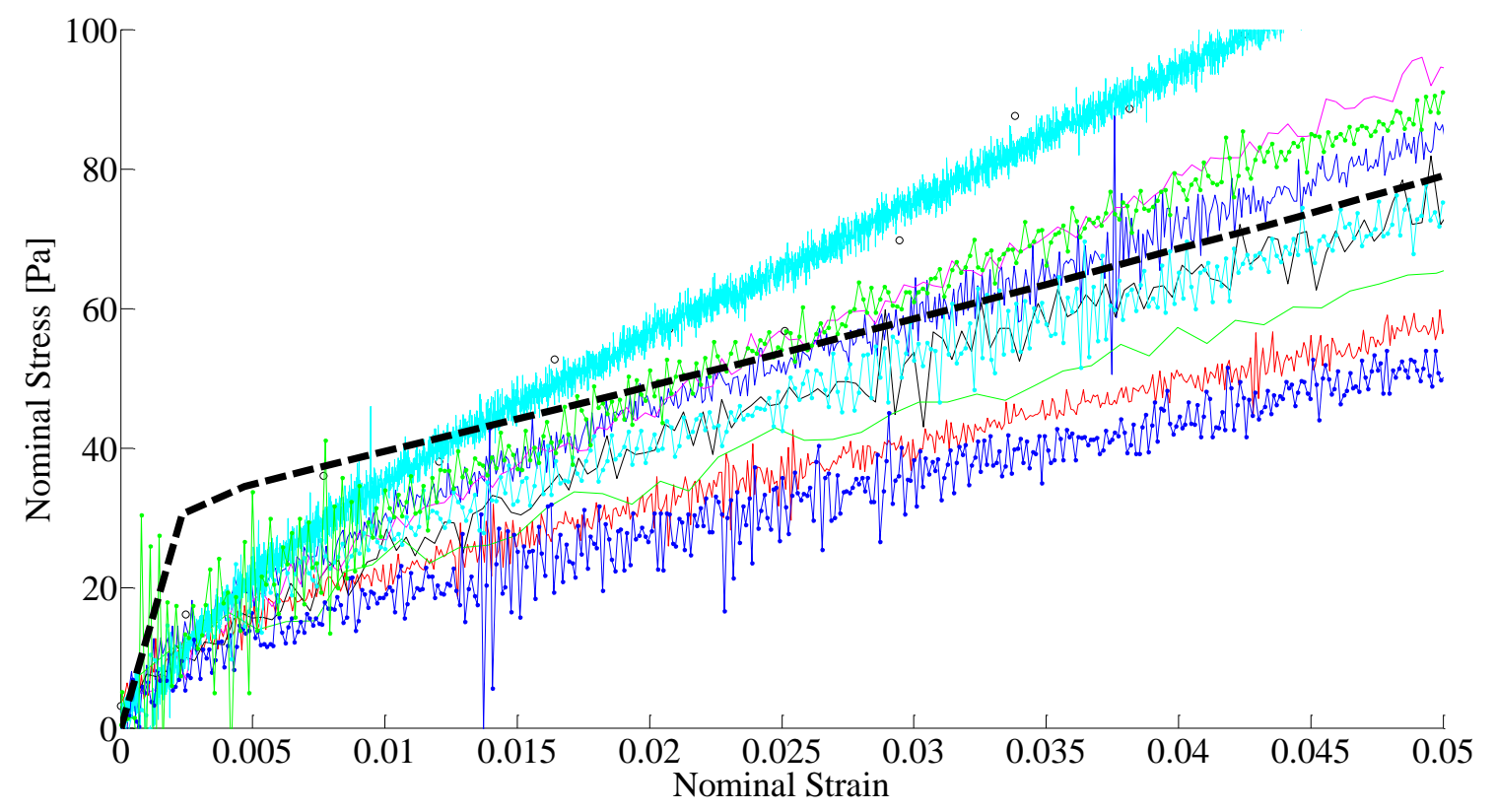

Figure 6.5 JS model tension results; low strain regime (model results shown in black) 


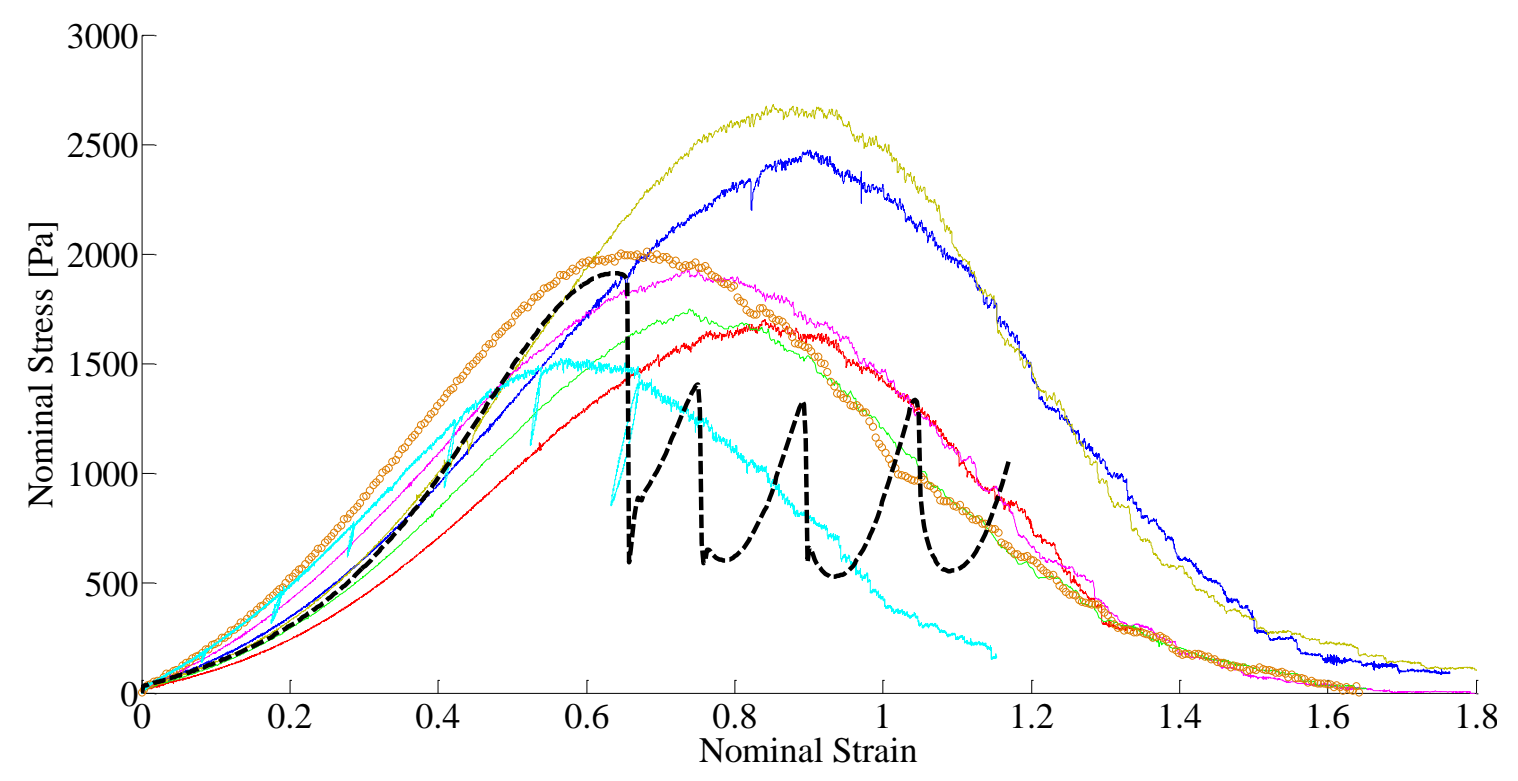

Figure 6.6 JS model tension results (model results shown in black)

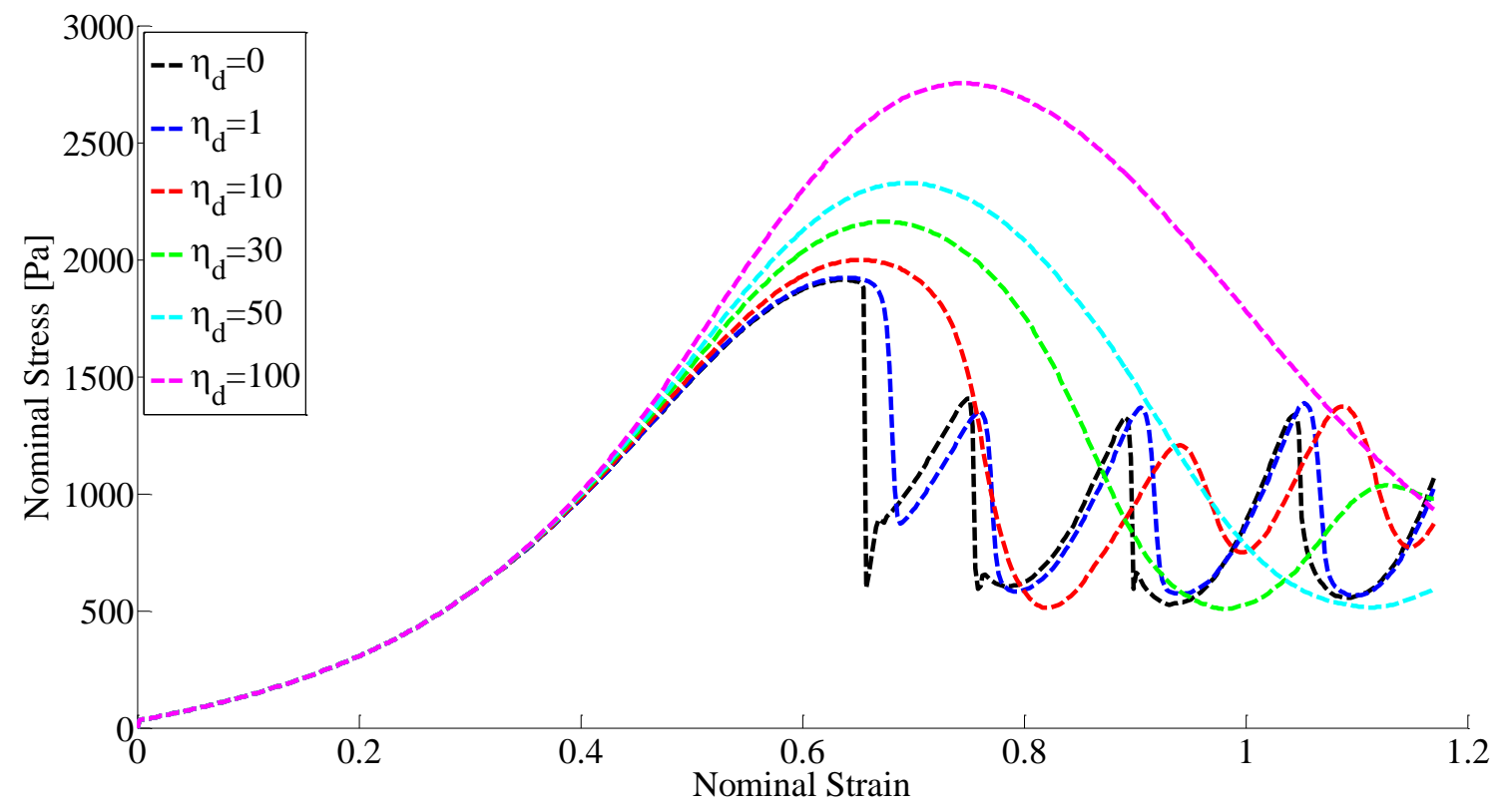

Figure 6.7 Effect of changing the damage viscosity parameter 


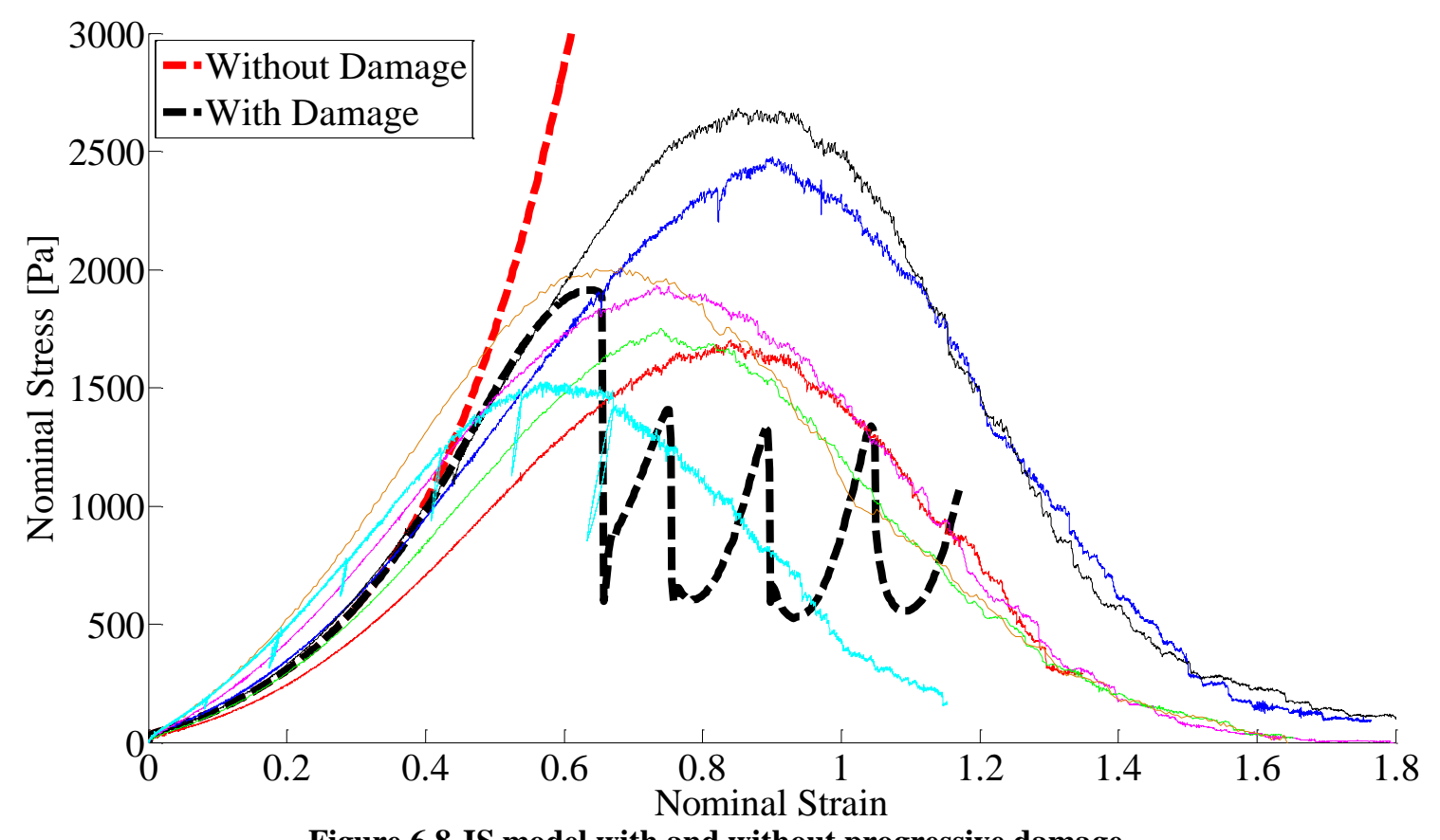

Figure 6.8 JS model with and without progressive damage.
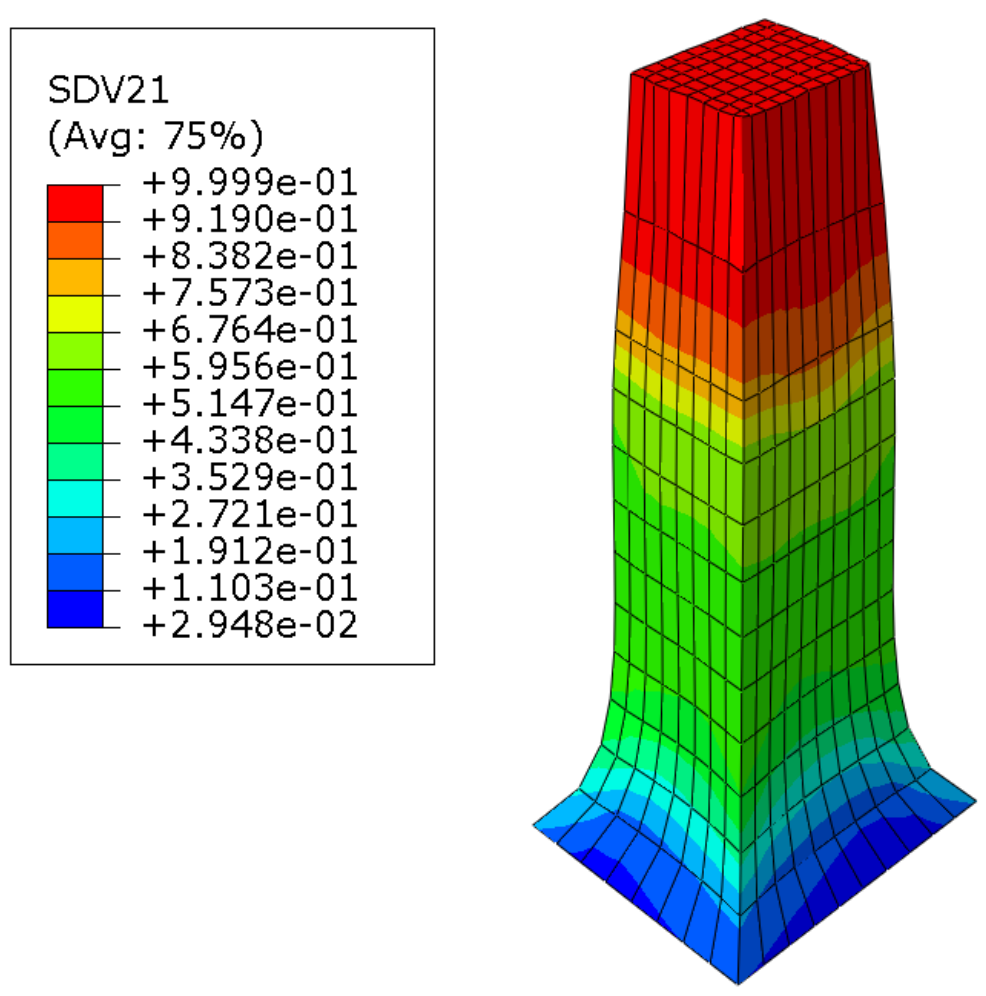

Figure 6.9 Damage localization in tension simulation (contours of damage shown) 


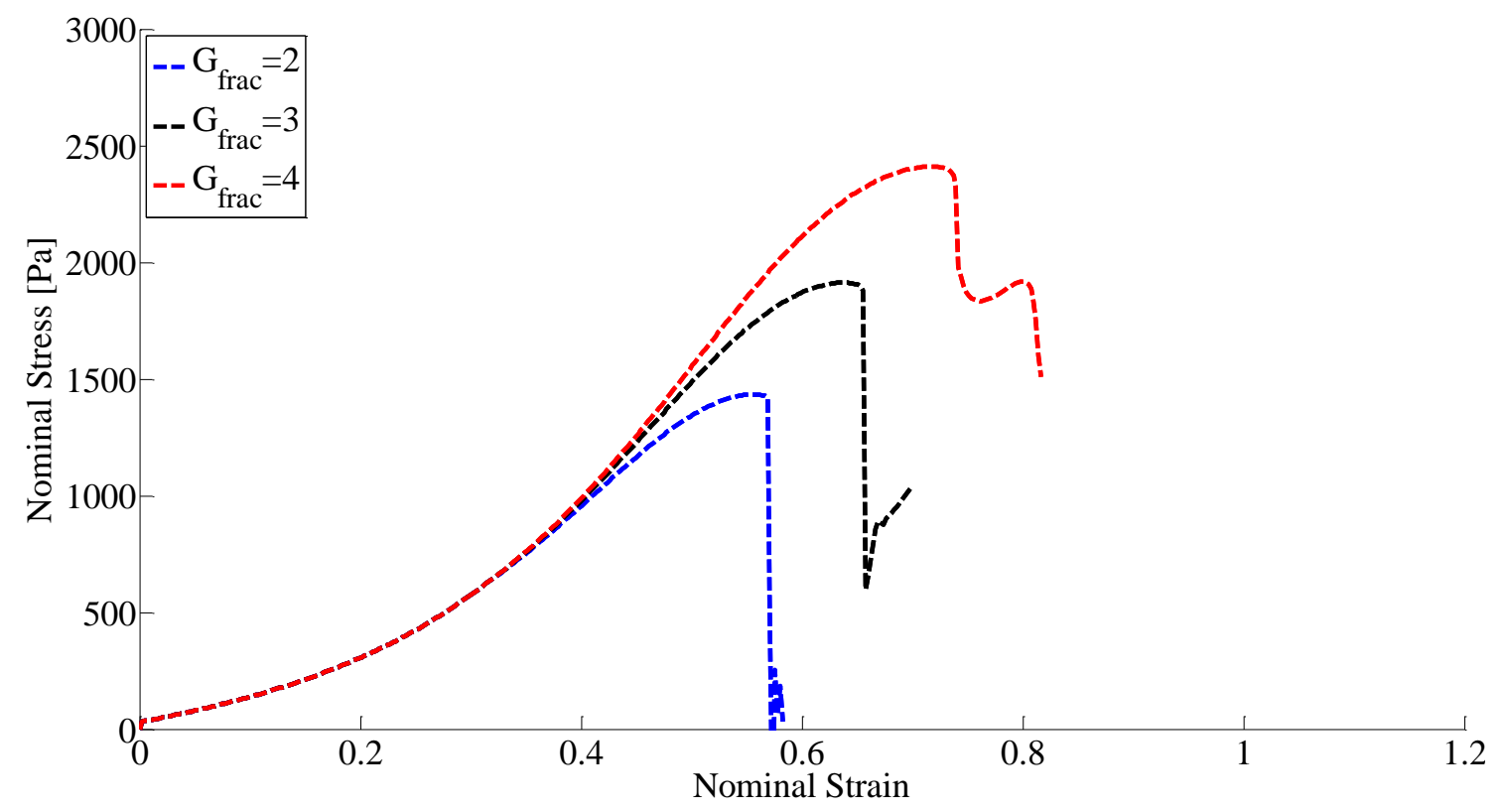

Figure 6.10 Effect of varying $\mathbf{G}_{\text {frac }}$

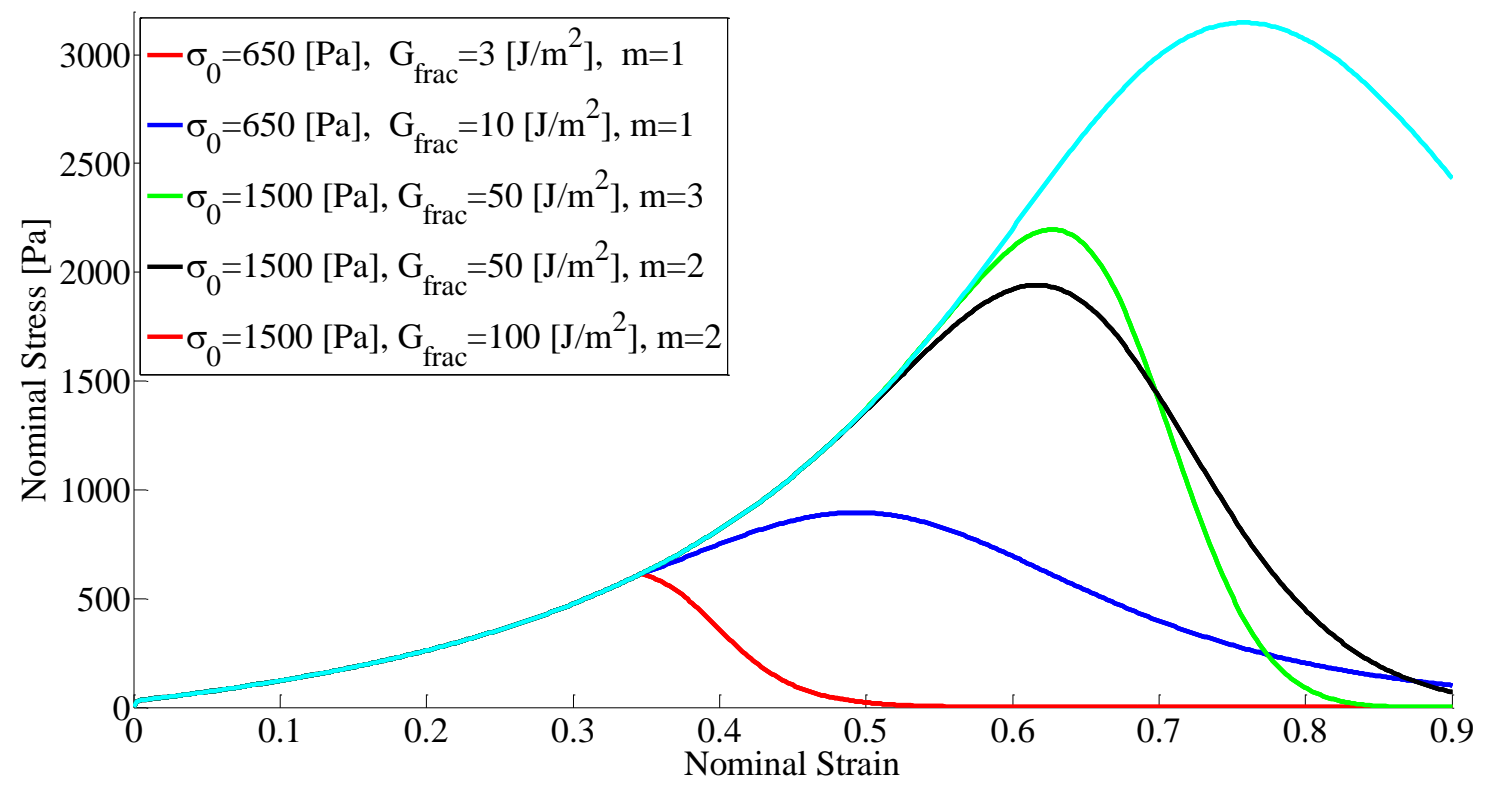

Figure 6.11 Single element simulations 


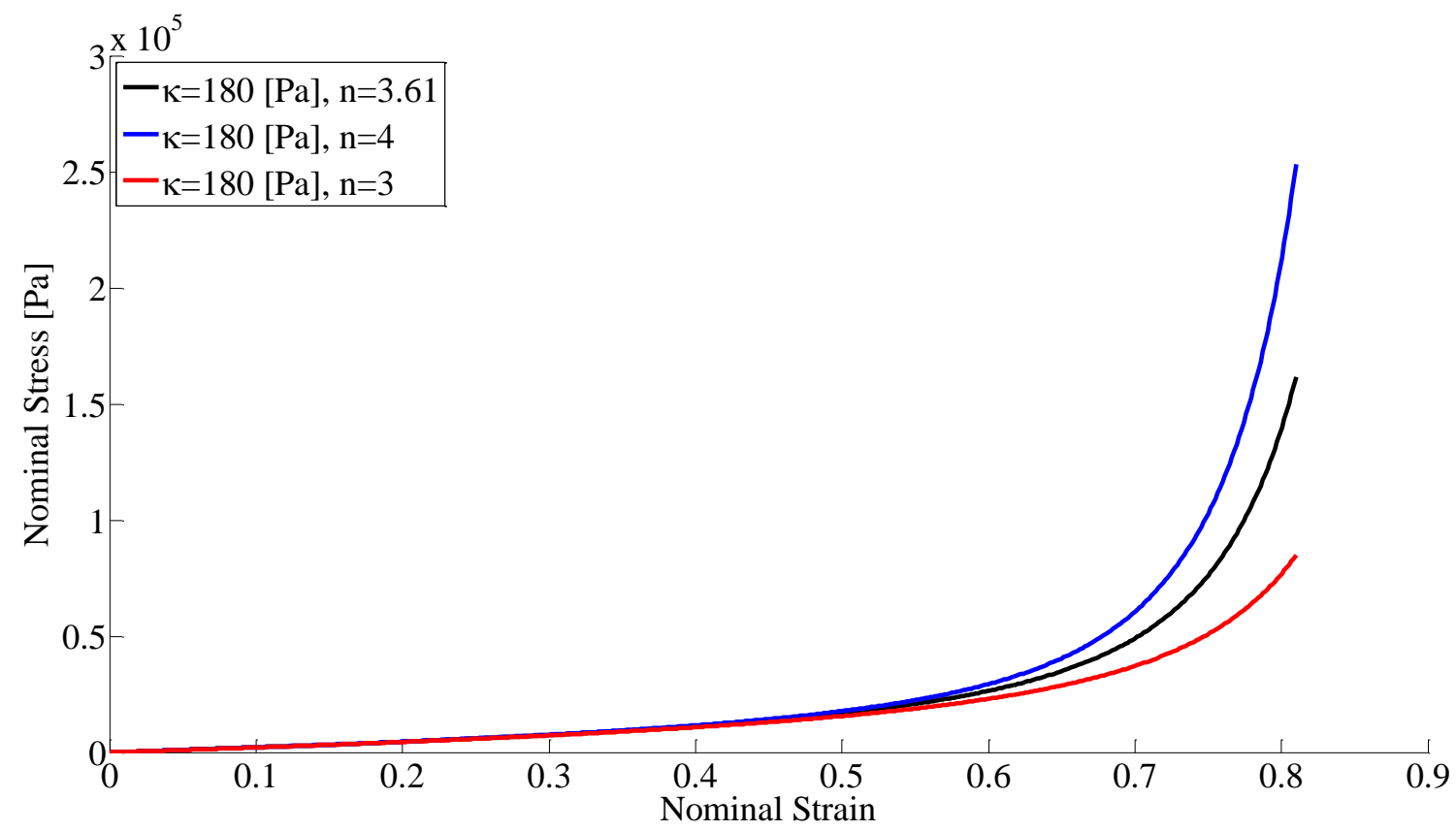

Figure 6.12 JS compression results 


\section{Conclusion}

The initial task of this thesis was to determine an effective lightweight armor system for a small robotic vehicle. The work presented here made progress toward that goal, but some deficiencies remain, namely, the lack of a finalized armor system capable of protecting against M855 ammunition. Nonetheless, low-density, thick-section, non-woven ballistic armors appear to be a promising material format, especially in the case of multi-hit performance. Polyester fiberfill was selected as the pilot test material, and showed surprisingly good ballistic performance against two types of $7.62 \mathrm{~mm}$ projectiles. The fact that a large-displacement armor, composed of relatively low performance fibers, was able to outperform approximately equivalent areal density hardened 1095 steel sheets versus ballistic threats bodes well for an armor system utilizing higher ballistic performance fibers.

There remains significant work to be done in characterizing large-displacement lightweight armor systems, especially in the ballistic regime. Good progress was made in the characterization of polyester fiberfill in the quasistatic regime; however, the behavior at low rates does not indicate the behavior at high rates. This is especially evident when comparing fiberfill's low strength and relatively low work-to-failure seen at quasistatic rates with its relatively good ballistic performance at high strain rates. This disparity is likely to be due, in some part, to the rate dependency of this material.

Avenues for improvement of fiberfill-based, large-displacement armor include incorporating hard outer face sheets to facilitate load spreading and projectile erosion (two areas where soft fiberfill armor is lacking), and utilization of high performance fibers as the constituent material. Investigation of both of these methods for improved ballistic performance would benefit greatly from numerical studies utilizing an accurate material model. 
The framework of the extended JS model presents a solid foundation for further refinement of an accurate fiberfill material model, but remains lacking in the area of damage modeling. As mentioned in Chapter 6, non-local averaging is necessary before adequate damage modeling can be accomplished. Furthermore, the model may benefit from the additions of anisotropic and/or rate dependent damage accounting for different modes of damage growth and rate dependence of material degradation. 


\section{References}

[1] T. W. Ipson and E. P. Wittrock, "Response of Non-Woven Synthetic Fiber Textiles to Ballistic Impact.," 1966.

[2] M. Ashby and C. Sammis, "The damage mechanics of brittle solids in compression," Pure and Applied Geophysics, vol. 133, pp. 489-521, 1990.

[3] S. Chocron, A. Pintor, F. Gálvez, C. Roselló, D. Cendón and V. Sanchez-Gálvez, "Lightweight polyethylene non-woven felts for ballistic impact applications: Material characterization," Composites Part B: Engineering, vol. 39, pp. 1240-1246, 2008.

[4] B. A. Cheeseman and T. A. Bogetti, "Ballistic impact into fabric and compliant composite laminates," Composite Structures, vol. 61, pp. 161-173, 2003.

[5] B. Compton, E. Gamble, V. Deshpande and F. Zok, "Damage development in an armor alumina impacted with ductile metal spheres," Journal of Mechanics of Materials and Structures, vol. 7, pp. 575-591, 2012.

[6] P. M. Cunniff, "Dimensionless parameters for optimization of textile-based body armor systems," 1999.

[7] N. David, X.-L. Gao and J. Zheng, "Ballistic resistant body armor: Contemporary and prospective materials and related protection mechanisms," Applied Mechanics Reviews, vol. 62, p. 0802, 2009.

[8] B. A. Gama, T. A. Bogetti, B. K. Fink, C.-J. Yu, T. D. Claar and H. H. Eifert, "Aluminum foam integral armor: a new dimension in armor design," Composite Structures, vol. 52, pp. 
381-395, 2001.

[9] E. A. Gamble, B. G. Compton, V. S. Deshpande, A. G. Evans and F. W. Zok, "Damage Development in an Armor Ceramic Under Quasi-Static Indentation," Journal of the American Ceramic Society, vol. 94, pp. s215-s225, 2011.

[10] J. Gillespie, A. Monib and L. Carlsson, "Damage tolerance of thick-section S-2 glass fabric composites subjected to ballistic impact loading," Journal of Composite Materials, vol. 37, pp. 2131-2147, 2003.

[11] R. Zaera and V. Sánchez-Gálvez, "Analytical modelling of normal and oblique ballistic impact on ceramic/metal lightweight armours," International Journal of Impact Engineering, vol. 21, pp. 133-148, 1998.

[12] D. M. Davis, "Finite Element Modeling of Ballistic Impact on a Glass Fiber Composite Armor," 2012.

[13] N. Naik and P. Shrirao, "Composite structures under ballistic impact," Composite structures, vol. 66, pp. 579-590, 2004.

[14] H. N. Wadley, T. Børvik, L. Olovsson, J. J. Wetzel, K. P. Dharmasena, O. S. Hopperstad, V. Deshpande and J. W. Hutchinson, "Deformation and fracture of impulsively loaded sandwich panels," Journal of the Mechanics and Physics of Solids, 2012.

[15] B. K. Fink, "Performance metrics for composite integral armor," Journal of Thermoplastic Composite Materials, vol. 13, pp. 417-431, 2000.

[16] H. Hopkins and H. Kolsky, "Mechanics of Hypervelocity Impact of Solids," 1960. 
[17] W. Goldsmith and J. Frasier, "Impact: The theory and physical behavior of colliding solids," Journal of Applied Mechanics, vol. 28, p. 639, 1961.

[18] M. E. Backman and W. Goldsmith, "The mechanics of penetration of projectiles into targets," International Journal of Engineering Science, vol. 16, pp. 1-99, 1978.

[19] C. J. Yungwirth, J. OConnor, A. Zakraysek, V. S. Deshpande and H. N. Wadley, "Explorations of hybrid sandwich panel concepts for projectile impact mitigation," Journal of the American Ceramic Society, vol. 94, pp. s62-s75, 2011.

[20] W. Johnson, N. Chitkara, A. Ibrahim and A. Dasgupta, "Hole flanging and punching of circular plates with conically headed cylindrical punches," The Journal of Strain Analysis for Engineering Design, vol. 8, pp. 228-241, 1973.

[21] G. Corbett, S. Reid and W. Johnson, "Impact loading of plates and shells by free-flying projectiles: a review," International Journal of Impact Engineering, vol. 18, pp. 141-230, 1996.

[22] B. Cheeseman, R. Jensen and C. Hoppel, "Protecting the future forces: Advanced materials and analysis enable robust composite armor," AMPTIAC Quarterly, vol. 8, pp. 37-43, 2004.

[23] S. Feli, M. Yas and M. Asgari, "An analytical model for perforation of ceramic/multilayered planar woven fabric targets by blunt projectiles," Composite Structures, vol. 93, pp. 548-556, 2011.

[24] B. K. Fink, T. A. Bogetti and B. Gama, "Application of Aluminum Foam for Stress-Wave Management in Lightweight Composite Integral Armor," 2001. 
[25] A. L. Florence and T. Ahrens, "Interaction of projectiles and composite armor," 1967.

[26] A. Tasdemirci and I. Hall, "Development of novel multilayer materials for impact applications: A combined numerical and experimental approach," Materials \& Design, vol. 30, pp. 1533-1541, 2009.

[27] A. Tasdemirci and I. Hall, "Experimental and modeling studies of stress wave propagation in multilayer composite materials: Low modulus interlayer effects," Journal of Composite Materials, vol. 39, pp. 981-1005, 2005.

[28] A. Tasdemirci and I. Hall, "Numerical and experimental studies of damage generation in multi-layer composite materials at high strain rates," International Journal of Impact Engineering, vol. 34, pp. 189-204, 2007.

[29] A. Tasdemirci, G. Tunusoglu and M. Güden, "The effect of the interlayer on the ballistic performance of ceramic/composite armors: Experimental and numerical study," International Journal of Impact Engineering, vol. 44, pp. 1-9, 2012.

[30] J. C. Smith, F. L. McCrackin and H. F. Schiefer, "Stress-Strain Relationships in Yarns Subjected to Rapid Impact Loading Part V: Wave Propagation in Long Textile Yarns Impacted Transversely," Textile Research Journal, vol. 28, pp. 288-302, 1958.

[31] D. Roylance and S.-S. Wang, "Penetration mechanics of textile structures," 1979.

[32] D. Roylance, "Stress wave propagation in fibres: effect of crossovers," Fibre Science and Technology, vol. 13, pp. 385-395, 1980.

[33] P. M. Cunniff, M. A. Auerbach, E. Vetter and D. J. Sikkema, "High performance "M5" 
fiber for ballistics/structural composites," 2002.

[34] R. R. Hegde, A. Dahiya, M. Kamath, R. Kotra and X. Gao, "Polyester Fibers," [Online]. Available: http://www.engr.utk.edu/mse/Textiles/Polyester\%20fiber.htm. [Accessed 2012].

[35] DuPont Advanced Fiber Systems, "KEVLAR Technical Guide," [Online]. Available: http://www2.dupont.com/Kevlar/en_US/assets/downloads/KEVLAR_Technical_Guide.pdf. [Accessed 2013].

[36] Honeywell Advanced Fibers and Composites, "Spectra Fibers Physical Properties," [Online]. Available: http://www.honeywelladvancedfibersandcomposites.com/products/fibers/.

[37] Hexcel, "Hex Tow Continuous Carbon Fiber Products," [Online]. Available: http://www.hexcel.com/Resources/Cont-Carbon-Fiber-Data-Sheets. [Accessed 2013].

[38] AGY, "S-2 Glass Products," [Online]. Available: http://www.agy.com/products/s2/continuous_filament.htm. [Accessed 2013].

[39] M. Karahan, "Comparison of ballistic performance and energy absorption capabilities of woven and unidirectional aramid fabrics," Textile Research Journal, vol. 78, pp. 718-730, 2008.

[40] R. C. Laible and M. C. Henry, "A Review of the Development of Ballistic Needle-punched Felts," 1969.

[41] S. Chocron, A. Pintor, D. Cendon, C. Rosello and V. Sanchez-Galvez, Characterization of Fraglight Non-Woven Felt and Simulation of FSPs Impact in it, 2002. 
[42] J. Hearle and A. Purdy, "Research on Energy Absorption by Nonwoven Fabrics," 1971.

[43] C.-C. Lin, C.-M. Lin, C.-C. Huang, C.-W. Lou, H.-H. Meng, C.-H. Hsu and J.-H. Lin, "Elucidating the Design and Impact Properties of Composite Nonwoven Fabrics with Various Filaments in Bulletproof Vest Cushion Layer," Textile Research Journal, vol. 79, pp. 268-274, 2009.

[44] M. Jacobs and J. V. Dingenen, "Ballistic protection mechanisms in personal armour," Journal of Materials Science, vol. 36, pp. 3137-3142, 2001.

[45] C. V. Wyk, "NOTE ON THE COMPRESSIBILITY OF WOOL," Journal of the Textile Institute Transactions, vol. 37, pp. T285-T292, 1946.

[46] L. Mezeix, C. Bouvet, J. Huez and D. Poquillon, "Mechanical behavior of entangled fibers and entangled cross-linked fibers during compression," Journal of Materials Science, vol. 44, pp. 3652-3661, 2009.

[47] S. Toll, "Packing mechanics of fiber reinforcements," Polymer Engineering \& Science, vol. 38, pp. 1337-1350, 1998.

[48] T. Komori and M. Itoh, "A new approach to the theory of the compression of fiber assemblies," Textile Research Journal, vol. 61, pp. 420-428, 1991.

[49] N. Pan, "A modified analysis of the microstructural characteristics of general fiber assemblies," Textile Research Journal, vol. 63, pp. 336-345, 1993.

[50] C. Barbier, R. Dendievel and D. Rodney, "Role of friction in the mechanics of nonbonded fibrous materials," Physical Review E, vol. 80, p. 016115, 2009. 
[51] G. A. Carnaby and N. Pan, "Theory of the compression hysteresis of fibrous assemblies," Textile Research Journal, vol. 59, pp. 275-284, 1989.

[52] C. Barbier, R. Dendievel and D. Rodney, "Numerical study of 3D-compressions of entangled materials," Computational Materials Science, vol. 45, pp. 593-596, 2009.

[53] N. B. Beil and W. W. Roberts, "Modeling and Computer Simulation of the Compressional Behavior of Fiber Assemblies Part I: Comparison to van Wyks Theory," Textile Research Journal, vol. 72, pp. 341-351, 2002.

[54] J. Masse, L. Salvo, D. Rodney, Y. Brechet and O. Bouaziz, "Influence of relative density on the architecture and mechanical behaviour of a steel metallic wool," Scripta Materialia, vol. 54, pp. 1379-1383, 2006.

[55] L. J. Gibson and M. F. Ashby, Cellular solids: structure and properties, Cambridge university press, 1999.

[56] G. T. Mase and G. E. Mase, Continuum mechanics for engineers, Crc Press, 2010.

[57] H. Cox, "The elasticity and strength of paper and other fibrous materials," British journal of applied physics, vol. 3, p. 72, 1952.

[58] M. A. Narter, S. K. Batra and D. R. Buchanan, "Micromechanics of three-dimensional fibrewebs: constitutive equations," Proceedings of the Royal Society of London.Series A: Mathematical, Physical and Engineering Sciences, vol. 455, pp. 3543-3563, 1999.

[59] S. Federico and T. C. Gasser, "Nonlinear elasticity of biological tissues with statistical fibre orientation," Journal of The Royal Society Interface, vol. 7, pp. 955-966, 2010. 
[60] J. E. Bischoff, E. M. Arruda and K. Grosh, "Finite element modeling of human skin using an isotropic, nonlinear elastic constitutive model," Journal of Biomechanics, vol. 33, pp. 645-652, 2000.

[61] E. M. Arruda and M. C. Boyce, "A three-dimensional constitutive model for the large stretch behavior of rubber elastic materials," Journal of the Mechanics and Physics of Solids, vol. 41, pp. 389-412, 1993.

[62] J. Bergström and M. Boyce, "Constitutive modeling of the large strain time-dependent behavior of elastomers," Journal of the Mechanics and Physics of Solids, vol. 46, pp. 931954, 1998.

[63] J. Palmer, C. Castro, M. Arslan and M. Boyce, "Constitutive models for the force-extension behavior of biological filaments," 2010.

[64] J. Skelton, "Frictional effects in fibrous assemblies," Textile Research Journal, vol. 44, pp. 746-752, 1974.

[65] N. Pan and G. A. Carnaby, "Theory of the shear deformation of fibrous assemblies," Textile Research Journal, vol. 59, pp. 285-292, 1989.

[66] J. Lambert and G. Jonas, "Towards standardization in terminal ballistics testing: velocity representation," 1976.

[67] J. O. Hallquist, "LS-DYNA theory manual," Livermore Software Technology Corporation, vol. 3, 2006.

[68] J. Planas, G. Guinea and M. Elices, "Constitutive model for fiber-reinforced materials with 
deformable matrices," Physical Review E, vol. 76, p. 041903, 2007.

[69] A. Ridruejo, C. González and J. LLorca, "A constitutive model for the in-plane mechanical behavior of nonwoven fabrics," International Journal of Solids and Structures, vol. 49, pp. 2215-2229, 2012.

[70] P. Jearanaisilawong, "A continuum model for needlepunched nonwoven fabrics," 2008.

[71] J. E. Bischoff, E. M. Arruda and K. Grosh, "A rheological network model for the continuum anisotropic and viscoelastic behavior of soft tissue," Biomechanics and modeling in mechanobiology, vol. 3, pp. 56-65, 2004.

[72] J. E. Bischoff, E. M. Arruda and K. Grosh, "Finite element simulations of orthotropic hyperelasticity," Finite Elements in Analysis and Design, vol. 38, pp. 983-998, 2002.

[73] S. G. Advani, "The use of tensors to describe and predict fiber orientation in short fiber composites," Journal of Rheology, vol. 31, p. 751, 1987.

[74] M. C. Boyce, G. Weber and D. M. Parks, "On the kinematics of finite strain plasticity," Journal of the Mechanics and Physics of Solids, vol. 37, pp. 647-665, 1989.

[75] A. Ridruejo, C. González and J. LLorca, "Micromechanisms of deformation and fracture of polypropylene nonwoven fabrics," International Journal of Solids and Structures, vol. 48, pp. 153-162, 2011.

[76] L. M. Kachanov, "Rupture time under creep conditions," International Journal of Fracture, vol. 97, pp. 11-18, 1999.

[77] J. Lemaitre, "How to use damage mechanics," Nuclear Engineering and Design, vol. 80, 
pp. 233-245, 1984.

[78] D. Krajcinovic, Damage mechanics, vol. 41, Access Online via Elsevier, 1996.

[79] A. Matzenmiller, J. Lubliner and R. Taylor, "A constitutive model for anisotropic damage in fiber-composites," Mechanics of Materials, vol. 20, pp. 125-152, 1995.

[80] B. Oh and Z. BAZANT, "Crack band theory for fracture of concrete," Materials and Structures, January-February, pp. 155-177, 1983.

[81] G. Duvaut and J. L. Lions, Les inéquations en mécanique et en physique, vol. 18, Dunod Paris, 1972.

[82] Dassault Systems Simulia, "ABAQUS theory manual," 2009.

[83] W. H. Press, Numerical recipes in Fortran 77: the art of scientific computing, vol. 1, Cambridge university press, 1992.

[84] M. Itskov, R. Dargazany and K. Hörnes, "Taylor expansion of the inverse function with application to the Langevin function," Mathematics and Mechanics of Solids, vol. 17, pp. 693-701, 2012.

[85] A. Cohen, "A Padé approximant to the inverse Langevin function," Rheologica Acta, vol. 30, pp. 270-273, 1991.

[86] J. Besson, D. Moinereau and D. Steglich, Local approach to fracture, Presses des MINES, 2006.

[87] S. Toll, "Note: On the tube model for fiber suspensions," Journal of Rheology, vol. 37, p. 
123, 1993.

[88] J. Bischoff, E. Arruda and K. Grosh, "A microstructurally based orthotropic hyperelastic constitutive law," Journal of applied mechanics, vol. 69, pp. 570-579, 2002.

[89] J. E. Bischoff, E. M. Arruda and K. Grosh, "A new constitutive model for the compressibility of elastomers at finite deformations," Rubber chemistry and technology, vol. 74, pp. 541-559, 2001.

[90] E. Kuhl, K. Garikipati, E. M. Arruda and K. Grosh, "Remodeling of biological tissue: mechanically induced reorientation of a transversely isotropic chain network," Journal of the Mechanics and Physics of Solids, vol. 53, pp. 1552-1573, 2005.

[91] P. Jearanaisilawong, "Investigation of deformation and failure mechanisms in woven and nonwoven fabrics under quasi-static loading conditions," 2004.

[92] E. H. Lee, Elastic-plastic deformation at finite strains, Stanford University, Divsion of Engineering Mechanics, 1968.

[93] E. Lee and D. Liu, "Finite-Strain Elastic-Plastic Theory with Application to Plane-Wave Analysis," Journal of Applied Physics, vol. 38, pp. 19-27, 1967.

[94] E. Kroner and C. Teodosiu, "Lattice defect approach to plasticity and viscoplasticity," Problems of plasticity.(A 75-13527 03-39) Leiden, Noordhoff International Publishing, 1974,, pp. 45-82, 1974.

[95] J. Mandel, "Thermodynamics and plasticity," Foundations of continuum thermodynamics, pp. 283-304, 1974. 
[96] J. Kratochvil, "On a finite strain theory of elastic-inelastic materials," Acta Mechanica, vol. 16, pp. 127-142, 1973.

[97] S. Nemat-Nasser, "On finite deformation elasto-plasticity," International Journal of Solids and Structures, vol. 18, pp. 857-872, 1982.

[98] A. Agah-Tehrani, E. Lee, R. Mallett and E. Onat, "The theory of elastic-plastic deformation at finite strain with induced anisotropy modeled as combined isotropic-kinematic hardening," Journal of the Mechanics and Physics of Solids, vol. 35, pp. 519-539, 1987.

[99] J. Simo and M. Ortiz, "A unified approach to finite deformation elastoplastic analysis based on the use of hyperelastic constitutive equations," Computer Methods in Applied Mechanics and Engineering, vol. 49, pp. 221-245, 1985.

[100] J. Simo and T. Hughes, "Computational inelasticity. 1998," New York.

[101] P. M. Naghdi, "A critical review of the state of finite plasticity," Zeitschrift für angewandte Mathematik und Physik ZAMP, vol. 41, pp. 315-394, 1990.

[102] J. Lubliner, "A maximum-dissipation principle in generalized plasticity," Acta Mechanica, vol. 52, pp. 225-237, 1984. 


\section{Appendix A. Complete Drop Test Results}

Table 4.3 Parameters for drop test experiments

\begin{tabular}{|c|c|c|c|}
\hline TestID & Drop height $[\mathrm{m}]$ & Impact velocity $[\mathrm{m} / \mathrm{s}]$ & Density of fiberfill $\left[\mathrm{kg} / \mathrm{m}^{3}\right]$ \\
\hline 1 & 0.77 & 3.91 & 66.7 \\
\hline 2 & 0.77 & 3.91 & 44.4 \\
\hline 3 & 0.35 & 2.62 & 66.7 \\
\hline 4 & 0.35 & 2.62 & 44.4 \\
\hline
\end{tabular}
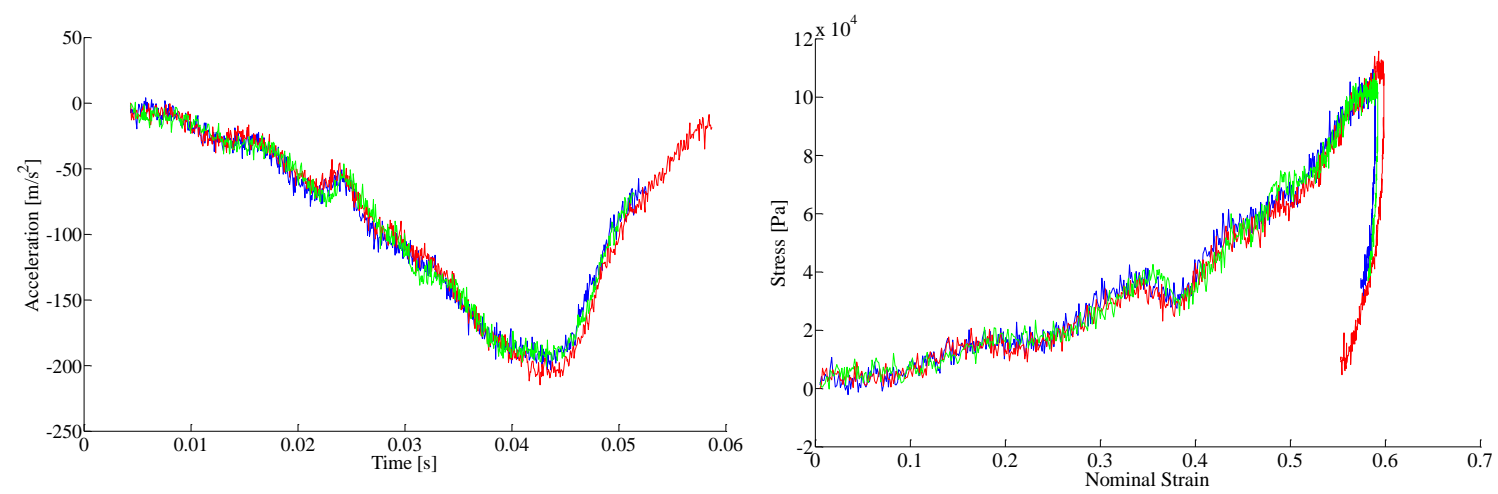

Figure A.1 Test ID \#1
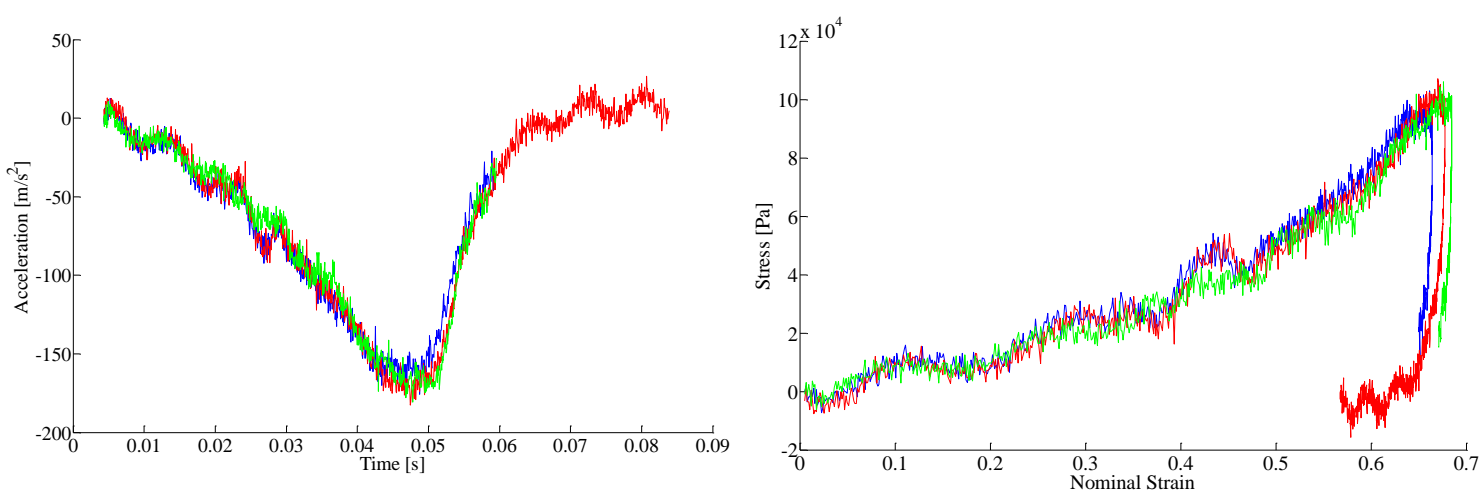

Figure A.2 Test ID \#2 

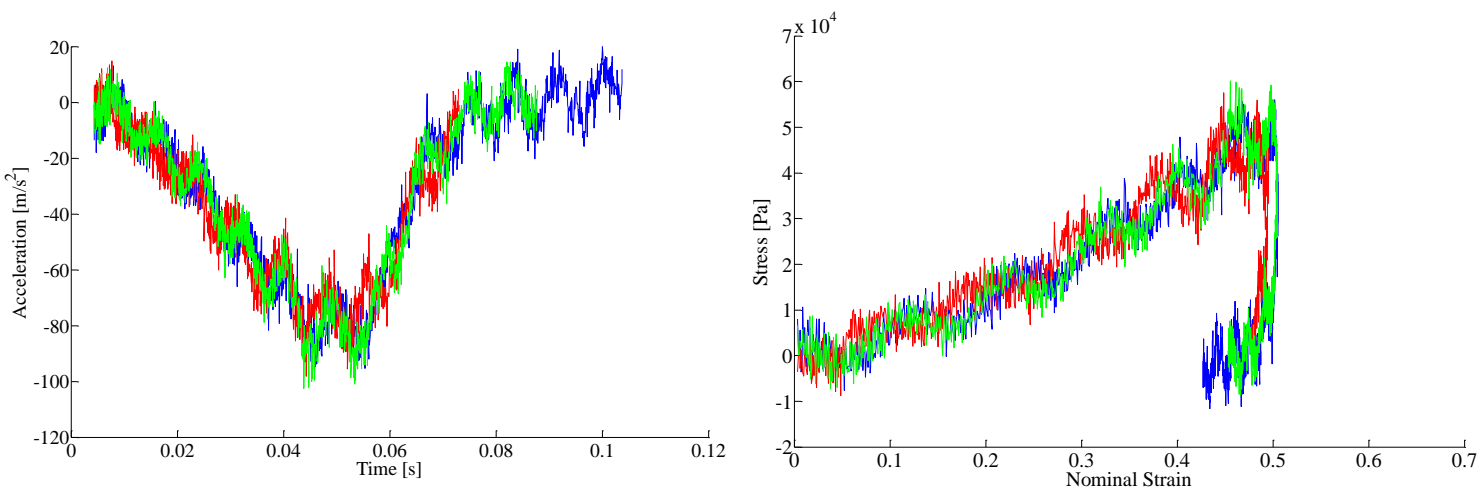

Figure A.3 Test ID \#3
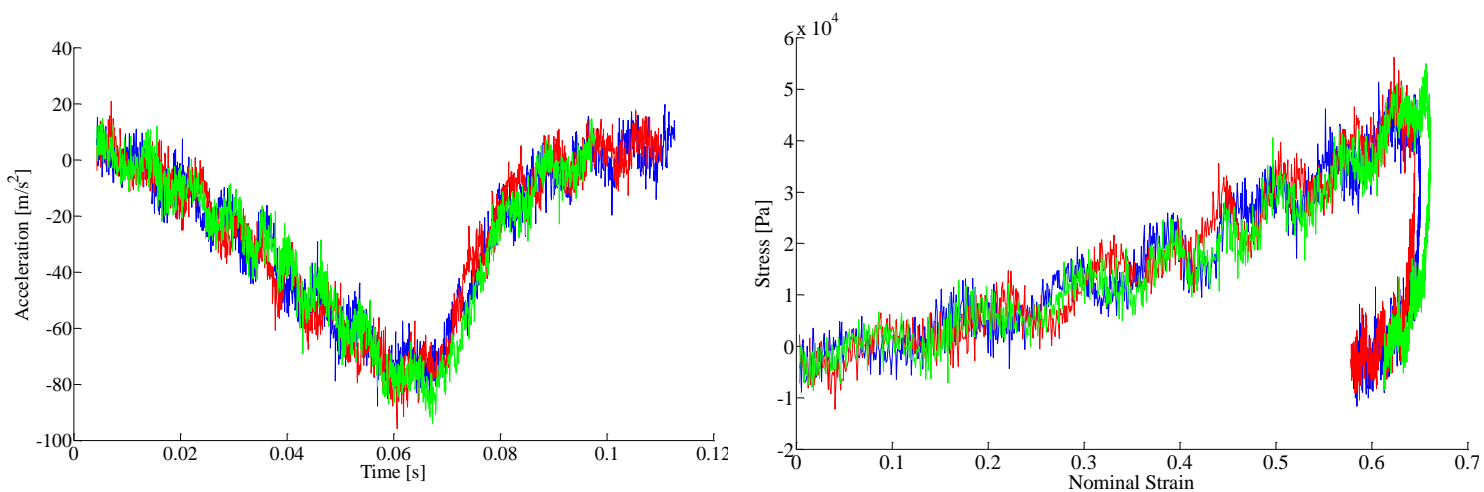

Figure A.4 Test ID \#4
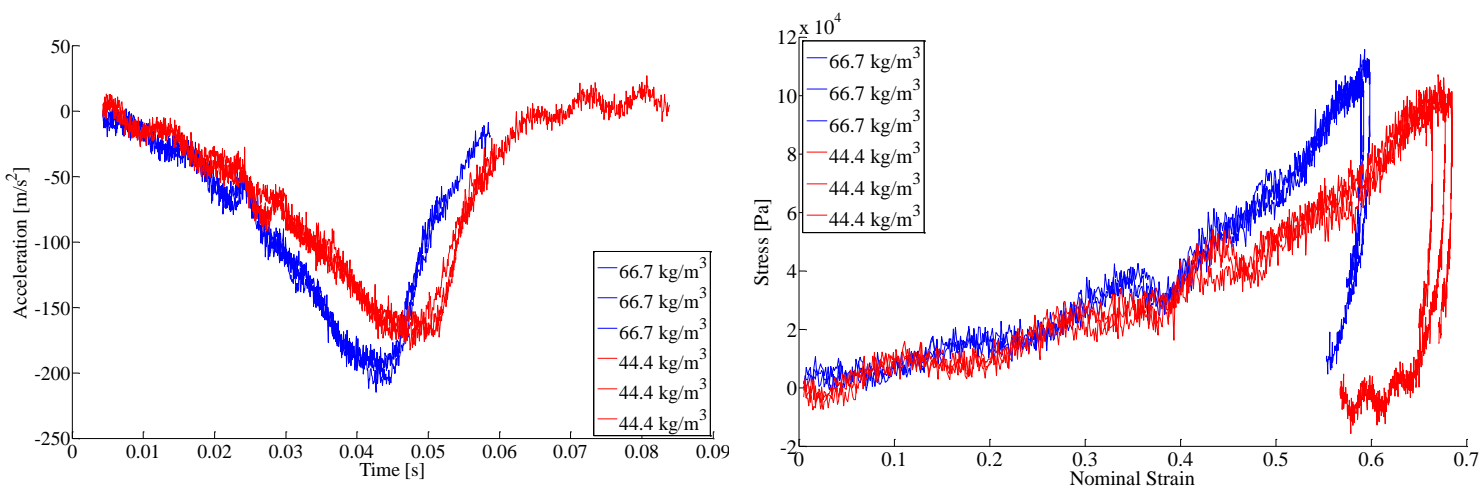

Figure A.5 Comparison of Test ID \#1 and Test ID \#2 fast impact; high versus low density 

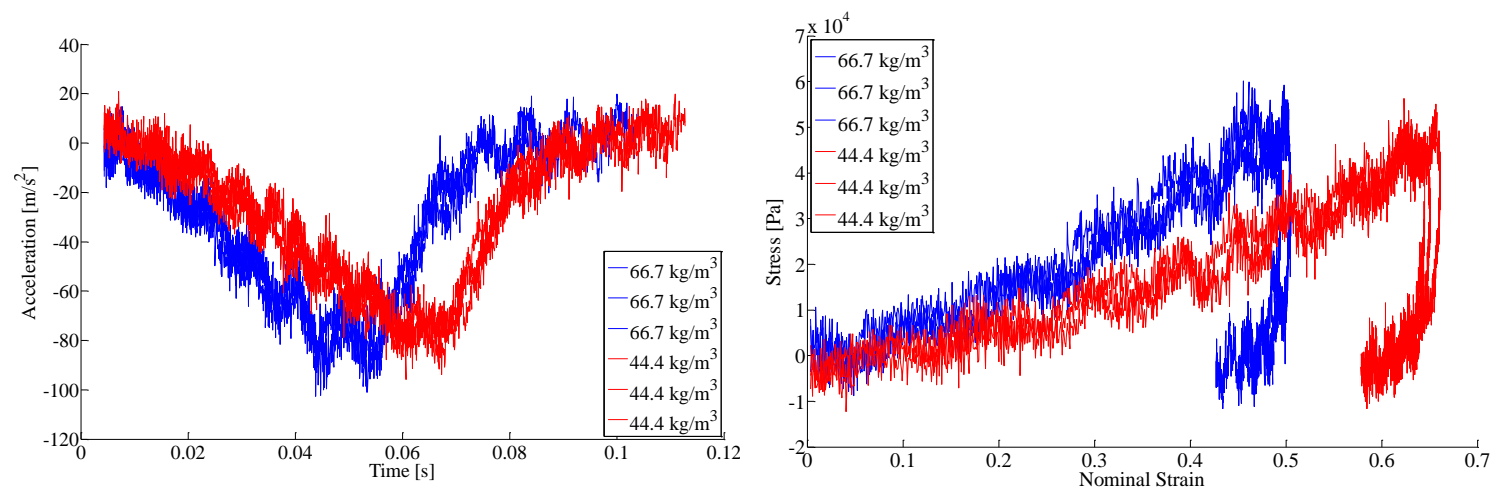

Figure A.6 Comparison Test ID \#3 and Test ID \#4 slow impact; high versus low density
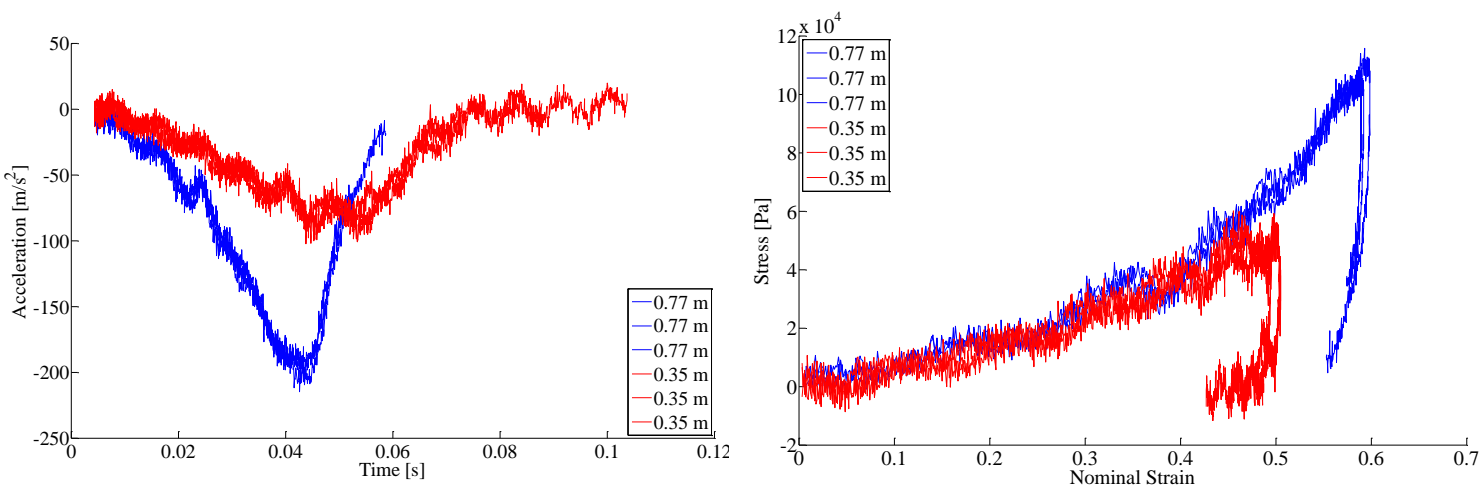

Figure A.7 Comparison of Test ID \#1 and Test ID \#3 high density; fast versus slow impact
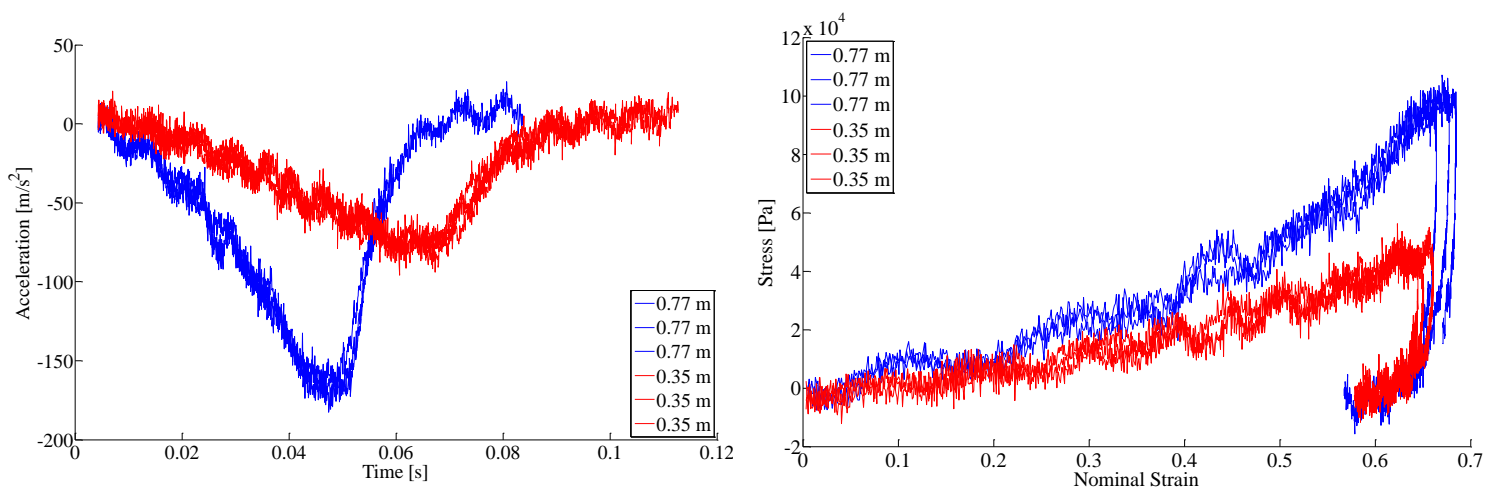

Figure A.8 Comparison of Test ID \#2 and Test ID \#4 low density; fast versus slow impact 


\section{Appendix B. JS Model Derivation}

This appendix is a recapitulation of the JS model derivation found in [70], with the additions proposed in section 6.2. Some of the finer points are emphasized; while material that is sufficiently covered in the original model derivation may be glossed over. This section is included here in hopes that, in conjunction with original paper by Jearanaisilawong, it may aid any future users of the JS model in understanding this model's derivation.

\section{B.1 Texture tensor structural analysis}

The number of points on a test fiber that are in contact with fibers in an arbitrary direction $\mathbf{p}^{\prime}$ is given in Toll's analysis of the compression of random fiber entanglements [47], [87]. First, a phantom fiber network in which fibers are able to interpenetrate is constructed. The average number of fiber centerlines intersecting a test fiber can be calculated by:

$$
\overline{\mathrm{N}}_{\mathrm{i}}=\mathrm{nl}^{2} \mathrm{fd}+\frac{1}{4} \pi \mathrm{nld}^{2}(\mathrm{~h}+1)
$$

Where $\mathrm{n}$ is the number of fibers per unit volume, $\bar{l}$ is the average length between crimps, $\mathrm{d}$ is the fiber diameter, and $\mathrm{f}$ and $\mathrm{h}$ are scalar invariants of the orientation distribution. For the number of intersections on a test fiber at a specified orientation $\mathbf{p}^{\prime}$ the invariants are:

$$
\begin{aligned}
& \mathrm{f}=\oint\left|\mathbf{p} \times \mathbf{p}^{\prime}\right| \psi(\mathbf{p}) \mathrm{d} \mathbf{p} \\
& \mathrm{h}=\oint\left|\mathbf{p} \cdot \mathbf{p}^{\prime}\right| \psi(\mathbf{p}) \mathrm{d} \mathbf{p}
\end{aligned}
$$

For the number of intersections with an average test fiber the invariants are then:

$$
\mathrm{f}=\oint \oint\left|\mathbf{p} \times \mathbf{p}^{\prime}\right| \psi\left(\mathbf{p}^{\prime}\right) \psi(\mathbf{p}) \mathrm{d} \mathbf{p}^{\prime} \mathrm{d} \mathbf{p}
$$




$$
\mathrm{h}=\oint \oint\left|\mathbf{p} \cdot \mathbf{p}^{\prime}\right| \psi\left(\mathbf{p}^{\prime}\right) \psi(\mathbf{p}) \mathrm{d} \mathbf{p}^{\prime} \mathrm{d} \mathbf{p}
$$

Where $\mathbf{p}$ is the orientation unit vector of a fiber and $\psi(\mathbf{p})$ is the fiber orientation distribution function. In a three dimensional randomly entangled assembly, $\mathrm{f}=\frac{\pi}{4}$ and $\mathrm{h}=\frac{1}{2}$. For a planar fiber splay $\mathrm{f}=\mathrm{h}=\frac{2}{\pi}$, and for aligned fibers $\mathrm{f}=0$ and $\mathrm{h}=1$.

The number of volume contacts is obtained by replacing $\mathrm{d}$ with $2 \mathrm{~d}$.

$$
\overline{\mathrm{N}}_{\mathrm{c}}=2 \mathrm{nl} \overline{\mathrm{l}}^{2} \mathrm{df}+\pi \mathrm{n} \overline{\mathrm{l}} \mathrm{d}^{2}(\mathrm{~h}+1)
$$

This can be written in terms of the fiber volume fraction, $\phi=\frac{1}{4} n \pi \mathrm{d}^{2} \overline{1}$ :

$$
\overline{\mathrm{N}}_{\mathrm{c}}=\frac{8}{\pi} \phi \overline{\mathrm{r}} \mathrm{f}+4 \phi(\mathrm{h}+1)
$$

Where $\overline{\mathrm{r}}=\frac{\overline{1}}{\mathrm{~d}}$ is the fiber aspect ratio. For high aspect ratios $\overline{\mathrm{r}} \gg 1$ and this reduces to:

$$
\overline{\mathrm{N}}_{\mathrm{c}}=\frac{8}{\pi} \phi \overline{\mathrm{r} f}
$$

The average spacing between contact points is:

$$
\bar{\lambda}=\frac{\overline{1}}{\overline{\mathrm{N}}_{\mathrm{c}}}
$$

Which for non-aligned slender fibers reduces to:

$$
\bar{\lambda}=\frac{\pi \mathrm{d}}{8 \phi \mathrm{f}}
$$

The interjunction distance in each material direction is a product of the junction-to-junction distance distribution, and the average inter-junction distance $\bar{\lambda}$ : 


$$
\lambda(\mathbf{p})=g(\mathbf{p}) \bar{\lambda}
$$

Eq B.11

using the relations:

$$
\begin{aligned}
& g(\mathbf{p})=\underline{\mathbf{G}}:(\mathbf{p} \otimes \mathbf{p}) \\
& \psi(\mathbf{p})=\underline{\boldsymbol{\Psi}}:(\mathbf{p} \otimes \mathbf{p})
\end{aligned}
$$

Where $\mathrm{g}(\mathbf{p})$ is the junction-to-junction distribution, $\underline{\mathbf{G}}$ is the second order texture tensor, and $\underline{\boldsymbol{\Psi}}$ is the second order orientation distribution tensor.

$$
\underline{\mathbf{G}}:(\mathbf{p} \otimes \mathbf{p})=\frac{\pi \mathrm{d}}{8 \phi \bar{\lambda}}\left(\oint \oint\left|\mathbf{p} \times \mathbf{p}^{\prime}\right| \underline{\Psi}:\left(\mathbf{p}^{\prime} \otimes \mathbf{p}^{\prime}\right) \underline{\Psi}:(\mathbf{p} \otimes \mathbf{p}) \mathrm{d} \mathbf{p}^{\prime} \mathrm{d} \mathbf{p}\right)^{-1}
$$

This defines a complex relationship between the orientation distribution and the texture tensor, but, in theory, could be determined via experimental means.

$$
\underline{\mathbf{G}}=\underline{\mathbf{F}} \underline{\mathbf{G}}_{0} \underline{\mathbf{F}}^{\mathrm{T}}
$$

\section{B.2 JS Elastic Model}

Fiber junction points are assumed to deform in an affine manner with the macroscopic continuum strain. The texture tensor transforms according to:

$$
\underline{\mathbf{G}}=\underline{\mathbf{F}} \underline{\mathbf{G}}_{0} \underline{\mathbf{F}}^{\mathrm{T}}
$$

Eq B.16

The fiber stretch in this framework is:

$$
\lambda_{\mathrm{f}}=\sqrt{\frac{\operatorname{tr}\left(\underline{\mathbf{F}} \underline{\mathbf{G}}_{0} \underline{\mathbf{F}}^{\mathrm{T}}\right)}{\operatorname{tr}\left(\underline{\mathbf{G}}_{0}\right)}}
$$

Thus, the fiber stretch is biased by the texture tensor according to the fiber orientation and junction-to-junction distance.

The strain energy function describing the fiber stretch is: 


$$
\Psi_{\text {fiber }}=\eta_{0} \eta \lambda_{1}^{2}\left(\left(\frac{\lambda_{\mathrm{f}}}{\lambda_{1}}\right) \beta\{\chi\}+\ln \left[\frac{\beta\{\chi\}}{\sinh (\beta\{\chi\})}\right]\right)+\eta_{0} \Psi_{0}
$$

Where $\eta_{0}$ is a scaling term with units of energy, $\eta$ is the length of fiber in the control volume, $\lambda_{1}$ is the locking stretch, $\chi=\lambda_{\mathrm{f}} / \lambda_{1}$ is the stretch ratio, $\beta\{\cdot\}$ is the inverse Langevin function, and $\Psi_{0}$ is a normalizing energy that forces the strain energy to equal zero in the unstretched configuration.

The second Piola-Kirchoff stress is calculated by:

$$
\underline{\mathbf{S}}=2 \frac{\partial \Psi_{\text {fiber }}}{\partial \underline{\mathbf{C}}}
$$

Where:

$$
\frac{\partial \Psi_{\text {fiber }}}{\partial \underline{\mathbf{C}}}=\eta_{0} \eta \lambda_{1}^{2}\left[\chi \frac{\partial \beta\{\chi\}}{\partial \chi} \frac{\partial \chi}{\partial \lambda_{\mathrm{f}}} \frac{\partial \lambda_{\mathrm{f}}}{\partial \underline{\mathbf{C}}}+\frac{\beta\{\chi\}}{\lambda_{1}} \frac{\partial \lambda_{\mathrm{f}}}{\partial \underline{\mathbf{C}}}-\left(\operatorname{coth}(\beta\{\chi\})-\frac{1}{\beta\{\chi\}}\right) \frac{\partial \beta\{\chi\}}{\partial \chi} \frac{\partial \chi}{\partial \lambda_{\mathrm{f}}} \frac{\partial \lambda_{\mathrm{f}}}{\partial \underline{\mathbf{C}}}\right]
$$

However:

$$
\operatorname{coth}(\beta\{\chi\})-\frac{1}{\beta\{\chi\}}=\mathrm{L}\{\beta\{\chi\}\}=\chi
$$

Therefore:

$$
\begin{gathered}
\frac{\partial \Psi_{\text {fiber }}}{\partial \underline{\mathbf{C}}}=\eta_{0} \eta \lambda_{1}^{2} \frac{\partial \lambda_{\mathrm{f}}}{\partial \underline{\mathbf{C}}}\left[\frac{\beta\{\chi\}}{\lambda_{1}}+\chi \frac{\partial \beta\{\chi\}}{\partial \chi} \frac{\partial \chi}{\partial \lambda_{\mathrm{f}}}-\chi \frac{\partial \beta\{\chi\}}{\partial \chi} \frac{\partial \chi}{\partial \lambda_{\mathrm{f}}}\right] \\
\frac{\partial \Psi_{\text {fiber }}}{\partial \underline{\mathbf{C}}}=\eta_{0} \eta \lambda_{1} \frac{\partial \lambda_{\mathrm{f}}}{\partial \underline{\mathbf{C}}} \beta\{\chi\}
\end{gathered}
$$

Now, employing the relation:

$$
\begin{gathered}
\frac{\partial \lambda_{\mathrm{f}}}{\partial \underline{\mathbf{C}}}=\frac{1}{2 \lambda_{\mathrm{f}} \operatorname{tr}\left(\underline{\mathbf{G}}_{0}\right)} \underline{\mathbf{G}}_{0} \\
\underline{\mathbf{S}}=\frac{\eta_{0} \eta}{\operatorname{tr}\left(\underline{\mathbf{G}}_{0}\right)}\left(\frac{\lambda_{1}}{\lambda_{\mathrm{f}}}\right) \beta\{\chi\} \underline{\mathbf{G}}_{0}
\end{gathered}
$$


A repulsion term $\left(\Psi_{\text {repulsion }}\right)$ must be added to enforce a stress-free reference configuration, and prevent collapse of the material [88] to [90]. The contribution to the stress due to repulsion between fibers is described by:

$$
\underline{\mathbf{S}}_{\text {repulsion }}=\frac{-\eta_{0} \eta}{\operatorname{tr}\left(\underline{\mathbf{G}}_{0}\right)} \lambda_{1} \beta_{0} \underline{\mathbf{U}}^{-1} \underline{\mathbf{G}}_{0} \underline{\mathbf{U}}^{-\mathrm{T}}
$$

Where $\beta_{0}=\beta\left\{\frac{1}{\lambda_{1}}\right\}$ is the value of the inverse Langevin function of the fiber stretch ratio in the reference configuration.

The model does not account for all mechanisms of resistance to bulk compressibility (for instance, volume exclusion). In order to take these effects into account an isotropic term is added to the strain energy function of the JS model:

$$
\begin{gathered}
\Psi_{\text {bulk }}=\frac{1}{2}\left(\frac{-\mathrm{J}_{\kappa} J^{-n}}{-n+1}+J_{\kappa}\right) \\
2 \frac{\partial \Psi_{\text {bulk }}}{\partial \underline{\mathbf{C}}}=\frac{-\kappa}{-\mathrm{n}+1}\left(-n J J^{-n-1} \frac{\partial \mathbf{J}}{\partial \underline{\mathbf{C}}}+J^{-n} \frac{\partial \mathbf{J}}{\partial \underline{\mathbf{C}}}\right)+\kappa \frac{\partial \mathbf{J}}{\partial \underline{\mathbf{C}}} \\
2 \frac{\partial \Psi_{\text {bulk }}}{\partial \underline{\mathbf{C}}}=\frac{-\kappa}{-n+1}(-n+1) J^{-n} \frac{\partial \mathbf{J}}{\partial \underline{\mathbf{C}}}+\kappa \frac{\partial \mathbf{J}}{\partial \underline{\mathbf{C}}}
\end{gathered}
$$

Using the identity:

$$
\begin{gathered}
\frac{\partial \mathrm{J}}{\partial \underline{\mathbf{C}}}=\underline{J}^{-1} \\
2 \frac{\partial \Psi_{\text {bulk }}}{\partial \underline{\mathbf{C}}}=-\kappa J\left(\mathrm{~J}^{-\mathrm{n}}-1\right) \underline{\mathbf{C}}^{-1}
\end{gathered}
$$

Where $\kappa$ is a bulk modulus term with units of pressure. The second Piola-Kirchoff stress arising from this term is:

$$
\underline{\mathbf{S}}_{\text {bulk }}=2 \frac{\partial \Psi_{\text {bulk }}}{\partial \underline{\mathbf{C}}}=-\kappa J\left(J^{-\mathrm{n}}-1\right) \underline{\mathbf{C}}^{-1}
$$


The second Piola-Kirchoff stress for the entire network including all contributions is:

$$
\begin{gathered}
\underline{\mathbf{S}}=\underline{\mathbf{S}}_{\text {stretch }}+\underline{\mathbf{S}}_{\text {repulsion }}+\underline{\mathbf{S}}_{\text {bulk }} \\
\underline{\mathbf{S}}=\frac{\eta_{0} \eta}{\operatorname{tr}\left(\underline{\mathbf{G}}_{0}\right)}\left(\left(\frac{\lambda_{1}}{\lambda_{\mathrm{f}}}\right) \beta\{\chi\} \underline{\mathbf{G}}_{0}-\lambda_{1} \beta_{0} \underline{\mathbf{U}}^{-1} \underline{\mathbf{G}}_{0} \underline{\mathbf{U}}^{-\mathrm{T}}\right)-\kappa \mathrm{J}\left(\mathrm{J}^{-\mathrm{n}}-1\right) \underline{\mathbf{C}}^{-1}
\end{gathered}
$$

Eq B.33

Eq B.34

This is related to the Cauchy stress by the tensorial push forward operation:

$$
\underline{\boldsymbol{\sigma}}=\frac{1}{\mathrm{~J}} \underline{\mathbf{F}} \underline{\mathbf{S}}^{\mathrm{F}}
$$

Finally the constitutive relationship is expressed as:

$$
\underline{\boldsymbol{\sigma}}=\frac{\mu}{\mathrm{J} \operatorname{tr}\left(\underline{\mathbf{G}}_{0}\right)}\left(\frac{\lambda_{1}}{\lambda_{\mathrm{f}}} \beta\{\chi\} \underline{\mathbf{F}} \underline{\mathbf{G}}_{0} \underline{\mathbf{F}}^{\mathrm{T}}-\lambda_{1} \beta_{0} \underline{\mathbf{R}}_{\mathbf{\mathbf { G }}} \underline{\mathbf{R}}^{\mathrm{T}}\right)-\kappa\left(\mathrm{J}^{\mathrm{-n}}-1\right) \underline{\mathbf{I}}
$$

Here $\mu=\eta \eta_{0}$ is a modulus parameter with units of pressure.

\section{B.3 Inelastic extension of the JS model}

The elastic JS model accounts for the recoverable deformation of fibers with an arbitrary orientation distribution, and tracks the change in orientation via a push forward of the texture tensor. The deformation of fiberfill is dominated, however, by inelastic effects leading to irrecoverable changes in the texture tensor [91]. These effects are captured via finite strain inelasticity.

A multiplicative split of the deformation gradient is utilized in the inelastic formulation of the JS model. Multiplicative decompositions of this type have been considered in [92] to [102], among others. This split relies on the concept of an intermediate configuration, where the intermediate configuration amounts to an unloading of all neighborhoods of the body (Figure B.1). This is described by: 


$$
\underline{\mathbf{F}}=\underline{\mathbf{F}}^{\mathrm{e}} \underline{\mathbf{F}}^{\mathrm{i}}
$$

Where the superscript " $\mathrm{e}$ " refers to the elastic part of the total deformation gradient, and the superscript "i" refers to the inelastic portion of the total deformation gradient. The Jacobian of transformation decomposes as:

$$
\begin{gathered}
\mathrm{J}=\operatorname{det}(\underline{\mathbf{F}})=\operatorname{det}\left(\underline{\mathbf{F}}^{\mathrm{e}} \underline{\mathbf{F}}^{\mathrm{i}}\right)=\operatorname{det}\left(\underline{\mathbf{F}}^{\mathrm{e}}\right) \operatorname{det}\left(\underline{\mathbf{F}}^{\mathrm{i}}\right)=\mathrm{J}^{\mathrm{e}} \mathrm{J}^{\mathrm{i}} \\
\mathrm{J}^{\mathrm{e}}=\operatorname{det}\left(\underline{\boldsymbol{F}}^{\mathrm{e}}\right) \\
\mathrm{J}^{\mathrm{i}}=\operatorname{det}\left(\underline{\boldsymbol{F}}^{\mathrm{i}}\right)
\end{gathered}
$$

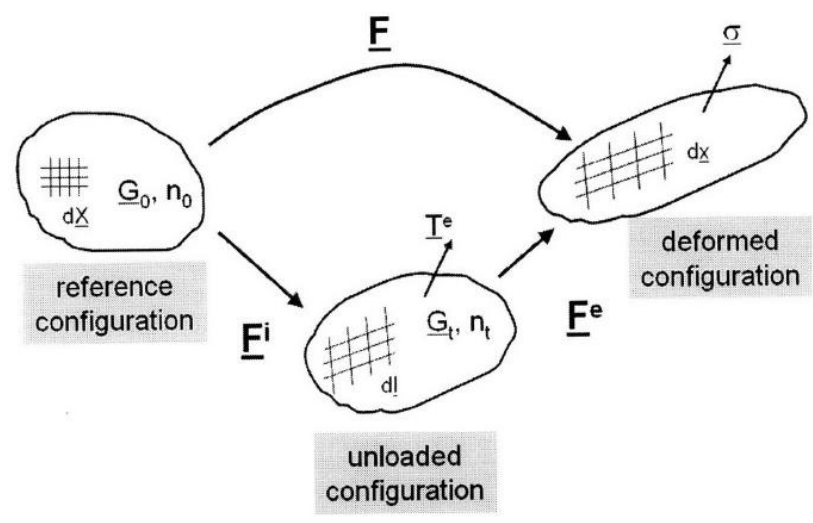

Figure B.1 Kroner-Lee Split of the deformation gradient [70]

The deformation gradients can be spectrally decomposed to:

$$
\begin{aligned}
& \underline{\mathbf{F}}^{\mathrm{e}}=\underline{\mathbf{R}}^{\mathrm{e}} \underline{\mathbf{U}}^{\mathrm{e}} \\
& \underline{\mathbf{F}}^{\mathrm{i}}=\underline{\mathbf{R}}^{\mathrm{i}} \underline{\mathbf{U}}^{\mathrm{i}}
\end{aligned}
$$

Eq B.41

Eq B.42

Where $\underline{\mathbf{R}}^{\mathrm{e}}$ and $\underline{\mathbf{R}}^{\mathrm{i}}$ are the elastic and inelastic rotation tensors, and $\underline{\mathbf{U}}^{\mathrm{e}}$ and $\underline{\mathbf{U}}^{\mathrm{i}}$ are the elastic and inelastic right stretch tensors. The elastic, right Cauchy-Green deformation tensor is defined by:

$$
\underline{\mathbf{C}}^{\mathrm{e}}=\underline{\mathbf{F}}^{\mathrm{e}^{\mathrm{T}}} \underline{\mathbf{F}}^{\mathrm{e}}=\underline{\mathbf{U}}^{\mathrm{e}^{2}}
$$


The rate of deformation is:

$$
\underline{\mathbf{F}}=\underline{\mathbf{L}} \underline{\mathbf{F}}
$$

Eq B.44

Where $\underline{\mathbf{L}}$ is the velocity gradient with elastic and inelastic components:

$$
\underline{\mathbf{L}}=\underline{\mathbf{L}}^{\mathrm{e}}+\underline{\mathbf{F}}^{\mathrm{e}} \underline{\mathbf{L}}^{\mathrm{i}} \underline{\mathbf{F}}^{\mathrm{e}^{-1}}
$$

Where:

$$
\underline{\mathbf{L}}=\underline{\mathbf{F}}^{\mathrm{e}} \underline{\mathbf{F}}^{\mathrm{e}^{-1}} \text { and } \underline{\mathbf{L}}^{\mathrm{i}}=\underline{\mathbf{F}}^{\mathrm{i}} \underline{\mathbf{F}}^{\mathrm{i}^{-1}}
$$

The velocity gradients decompose to their symmetric and skew symmetric parts according to:

$$
\begin{gathered}
\underline{\mathbf{L}}=\frac{1}{2}\left(\underline{\mathbf{L}}+\underline{\mathbf{L}}^{\mathrm{T}}\right)+\frac{1}{2}\left(\underline{\mathbf{L}}-\underline{\mathbf{L}}^{\mathrm{T}}\right)=\underline{\mathbf{D}}+\underline{\mathbf{W}} \\
\underline{\mathbf{L}}^{\mathrm{e}}=\frac{1}{2}\left(\underline{\mathbf{L}}^{\mathrm{e}}+\underline{\mathbf{L}}^{\mathrm{e}^{\mathrm{T}}}\right)+\frac{1}{2}\left(\underline{\mathbf{L}}^{\mathrm{e}}-\underline{\mathbf{L}}^{\mathrm{e}^{\mathrm{T}}}\right)=\underline{\mathbf{D}}^{\mathrm{e}}+\underline{\mathbf{W}}^{\mathrm{e}} \\
\underline{\mathbf{L}}^{\mathrm{i}}=\frac{1}{2}\left(\underline{\mathbf{L}}^{\mathrm{i}}+\underline{\mathbf{L}}^{\mathrm{i}^{\mathrm{T}}}\right)+\frac{1}{2}\left(\underline{\mathbf{L}}^{\mathrm{i}}-\underline{\mathbf{L}}^{\mathrm{i}^{\mathrm{T}}}\right)=\underline{\mathbf{D}}^{\mathrm{i}}+\underline{\mathbf{W}}^{\mathrm{i}}
\end{gathered}
$$

Where $\underline{\mathbf{D}}$ is the symmetric rate of deformation tensor and $\underline{\mathbf{W}}$ is the skew spin tensor.

In the present model, the inelastic deformation gradient will be solved via an explicit update using the equation:

$$
\dot{\mathbf{F}}^{\mathrm{i}}=\underline{\mathbf{L}}^{\mathrm{i}} \underline{\mathbf{F}}^{\mathrm{i}}
$$

And the elastic portion is then solved from:

$$
\underline{\mathbf{F}}^{\mathrm{e}}=\underline{\mathbf{F}} \underline{\mathbf{F}}^{\mathrm{i}^{-1}}
$$


The second Piola-Kirchoff stresses in the reference and relaxed configurations, respectively, are calculated as:

$$
\underline{\mathbf{S}}=\mathbf{J} \underline{\mathbf{F}}^{-1} \underline{\boldsymbol{\sigma}} \underline{\mathbf{F}}^{-\mathrm{T}} \text { and } \underline{\mathbf{S}}^{\mathrm{e}}=\mathrm{J}^{\mathrm{e}} \underline{\mathbf{F}}^{\mathrm{e}^{-1}} \underline{\boldsymbol{\sigma}} \underline{\mathbf{F}}^{\mathrm{e}^{-\mathrm{T}}}
$$

The rate of internal mechanical work in the continuum can be referred to by the contraction of the Cauchy stress with the rate of deformation tensor, integrated over the volume of the body:

$$
W_{\text {internal }}=\int \underline{\mathbf{\sigma}}: \underline{\mathbf{D}} d v
$$

Utilizing the elastic-inelastic decomposition developed previously. This can be expressed as:

$$
\begin{gathered}
W_{\text {internal }}=\int \underline{\boldsymbol{\sigma}}: \operatorname{sym}\left(\underline{\mathbf{L}}^{\mathrm{e}}+\underline{\mathbf{F}}^{\mathrm{e}} \underline{\mathbf{L}}^{\mathrm{i}} \underline{\mathbf{F}}^{\mathrm{e}^{-1}}\right) d v \\
=\int \underline{\boldsymbol{\sigma}}: \underline{\mathbf{D}}^{\mathrm{e}} d v^{i}+\int \mathrm{J}^{\mathrm{e}}\left(\underline{\mathbf{F}}^{\mathrm{e}^{\mathrm{T}}} \underline{\boldsymbol{\sigma}} \underline{\mathbf{F}}^{\mathrm{e}^{-\mathrm{T}}}: \underline{\mathbf{L}}^{\mathrm{i}}\right) d v^{i} \\
=\int \frac{1}{2}\left(\underline{\mathbf{S}}^{\mathrm{e}}: \underline{\mathbf{C}}^{\mathrm{e}}\right) \mathrm{d} v^{\mathrm{i}}+\int\left(\underline{\mathbf{C}}^{\mathrm{e}} \underline{\mathbf{S}}^{\mathrm{e}}: \underline{\mathbf{L}}^{\mathrm{i}}\right) \mathrm{d} v^{\mathrm{i}} \\
W_{\text {internal }}=W_{\text {elastic }}+W_{\text {inelastic }}
\end{gathered}
$$

Here $d v^{i}=\mathrm{J}^{\mathrm{e}^{-1}} d v$ is the differential volume defined in the relaxed configuration, and $\underline{\dot{\mathbf{C}}}=2 \underline{\mathbf{F}}^{\mathrm{e}} \underline{\mathbf{D}}^{\mathrm{e}} \underline{\mathbf{F}}^{\mathrm{e}}$. In order to satisfy the second law of thermodynamics, the inelastic work done must be dissipative [95]:

$$
\underline{\underline{E}}: \underline{\mathbf{L}}^{\mathrm{i}} \geq 0
$$

Where:

$$
\underline{\Xi}=\underline{\mathbf{C}}^{\mathrm{e}} \underline{\mathbf{S}}^{\mathrm{e}}
$$

is the Mandel stress. 
The application of this theory to non-woven fabrics relies on the following assumptions:

1. The junctions in the fabric can be modeled as material points which displace in an affine manner with the continuum.

2. The fabric texture can be captured through a single structural tensor $\underline{\mathbf{G}}_{t}$ that evolves with time in an affine manner with $\underline{\mathbf{F}}^{i}$. The fabric anisotropy in the unloaded configuration at time $t$ can then be described in terms of the fabric texture tensor:

$$
\underline{\mathbf{G}}_{\mathrm{t}}=\underline{\mathbf{F}}^{\mathrm{i}}(t) \underline{\mathbf{G}}_{0} \underline{\mathbf{F}}^{\mathrm{i}^{\mathrm{T}}}(t)
$$

3. The elastic response of the network can be obtained by the constitutive relation proposed in the elastic JS model with the evolving texture tensor $\underline{\mathbf{G}}_{\mathrm{t}}$ replacing $\underline{\mathbf{G}}_{0}$, the texture tensor in the reference configuration.

4. Fibers do not undergo inelastic deformation, accumulate no damage, and do not slip from junction points. This ensures that the number of fiber segments and the average end-toend unstretched length of fiber segments in the body are constant.

The spectral representation of the evolving texture tensor is written as:

$$
\underline{\mathbf{G}}_{\mathrm{t}}=\left[\begin{array}{ccc}
\mathrm{A}_{\mathrm{t}}^{2} & 0 & 0 \\
0 & \mathrm{~B}_{\mathrm{t}}^{2} & 0 \\
0 & 0 & \mathrm{C}_{\mathrm{t}}^{2}
\end{array}\right]
$$

The average end-to-end unstretched fiber length $\mathrm{r}_{\mathrm{t}}$ in the unloaded configuration at time $t$ can be expressed as:

$$
r_{t}=\frac{1}{2} d_{t} \sqrt{A_{t}^{2}+B_{t}^{2}+C_{t}^{2}}
$$

And as a consequence of the $4^{\text {th }}$ constitutive assumption $r_{t}=$ constant $=r_{0}$ or: 


$$
\operatorname{tr}\left(\underline{\mathbf{G}}_{\mathrm{t}}\right)=\operatorname{tr}\left(\underline{\mathbf{G}}_{0}\right)=3
$$

Since the number of fiber segments in the body is constant, and the volume in the unloaded configuration scales with the inelastic Jacobian, the number of fiber segments in the unloaded configuration scales as:

$$
\eta_{\mathrm{t}}=\frac{\eta_{0}}{\mathrm{~J}^{\mathrm{i}}}
$$

According to the $3^{\text {rd }}$ constitutive assumption the Cauchy stress is:

$$
\underline{\mathbf{S}}^{\mathrm{e}}=\frac{\eta_{\mathrm{t}} \eta}{\operatorname{tr}\left(\underline{\mathbf{G}}_{\mathrm{t}}\right)}\left(\left(\frac{\lambda_{\mathrm{l}}}{\lambda_{\mathrm{f}}^{\mathrm{e}}}\right) \beta\left\{\chi^{\mathrm{e}}\right\} \underline{\mathbf{G}}_{\mathrm{t}}-\lambda_{1} \beta_{0} \underline{\mathbf{U}}^{\mathrm{e}^{-1}} \underline{\mathbf{G}}_{\mathrm{t}} \underline{\mathbf{U}}^{\mathrm{e}^{-\mathrm{T}}}\right)-\kappa \mathrm{J}^{\mathrm{e}}\left(\mathrm{J}^{\mathrm{J}^{\mathrm{n}}}-1\right) \underline{\mathbf{C}}^{\mathrm{e}^{-1}}
$$

Where the effective elastic stretch is:

$$
\lambda_{\mathrm{f}}^{\mathrm{e}}=\sqrt{\frac{\operatorname{tr}\left(\underline{\mathbf{F}}^{\mathrm{e}} \underline{\mathbf{G}}_{\mathrm{t}} \mathbf{F}^{\mathrm{e}^{\mathrm{T}}}\right)}{\operatorname{tr}\left(\underline{\mathbf{G}}_{\mathrm{t}}\right)}}
$$

And $\chi^{\mathrm{e}}=\frac{\lambda_{\mathrm{f}}^{\mathrm{e}}}{\lambda_{1}}$ is the elastic stretch ratio.

The Cauchy stress is then found from a push forward to the relaxed configuration:

$$
\begin{gathered}
\underline{\boldsymbol{\sigma}}=\frac{1}{\mathrm{~J}} \underline{\mathbf{F}}^{\mathrm{e}} \underline{\mathbf{S}}^{\mathrm{e}} \underline{\mathbf{F}}^{\mathrm{e}^{\mathrm{T}}} \\
\underline{\boldsymbol{\sigma}}=\frac{\mu}{\mathrm{J} \operatorname{tr}\left(\underline{\mathbf{G}}_{\mathrm{t}}\right)}\left(\frac{\lambda_{1}}{\lambda_{\mathrm{f}}^{\mathrm{e}}} \beta\left\{\chi^{\mathrm{e}}\right\} \underline{\mathbf{F}}^{\mathrm{e}} \underline{\mathbf{G}}_{\mathrm{t}} \underline{\mathbf{F}}^{\mathrm{e}^{\mathrm{T}}}-\lambda_{1} \beta_{0} \underline{\mathbf{R}}^{\mathrm{e}} \underline{\mathbf{G}}_{\mathrm{t}} \underline{\mathbf{R}}^{\mathrm{e}^{\mathrm{T}}}\right)-\kappa\left(\mathrm{J}^{\mathrm{e}}-1\right) \underline{\mathbf{I}}
\end{gathered}
$$

Here $\eta_{t}=\frac{\eta_{0}}{\mathrm{~J}^{\mathrm{i}}}$ therefore $\frac{\eta_{0} \eta}{\mathrm{j}^{\mathrm{i}} \mathrm{j}^{\mathrm{e}}}=\frac{\mu}{\mathrm{J}}$

For irrecoverable deformation to occur, fibers must overcome interfiber friction and volume exclusion resulting from high levels of fiber volume fraction. Experimentally, the rate of 
structural evolution decreases as the fibers become more aligned in the principal loading direction.

Following the $2^{\text {nd }}$ constitutive assumption the rate of evolution of $\underline{\mathbf{G}}_{\mathrm{t}}$ and $\underline{\mathbf{L}}^{\mathrm{i}}$ are related by:

$$
\begin{gathered}
\dot{\mathbf{G}}_{\mathrm{t}}=\dot{\mathbf{F}}^{\mathrm{i}} \underline{\mathbf{G}}_{0} \underline{\mathbf{F}}^{\mathrm{i}^{\mathrm{T}}}+\underline{\mathbf{F}}^{\mathrm{i}} \underline{\mathbf{G}}_{0} \dot{\mathbf{F}}^{\mathrm{i}^{\mathrm{T}}} \\
\underline{\dot{\mathbf{G}}}_{\mathrm{t}}=\underline{\mathbf{L}}^{\mathrm{i}} \underline{\mathbf{F}}^{\mathrm{i}} \underline{\mathbf{G}}_{0} \underline{\mathbf{F}}^{\mathrm{i}^{\mathrm{T}}}+\underline{\mathbf{F}}^{\mathrm{i}} \underline{\mathbf{G}}_{0} \underline{\mathbf{F}}^{\mathrm{i}^{\mathrm{T}}} \underline{\mathbf{L}}^{\mathrm{i}^{\mathrm{T}}} \\
\underline{\dot{\mathbf{G}}}_{\mathrm{t}}=\underline{\mathbf{L}}^{\mathrm{i}} \underline{\mathbf{G}}_{\mathrm{t}}+\underline{\mathbf{G}}_{\mathrm{t}} \underline{\mathbf{L}}^{\mathrm{i} \mathrm{T}}
\end{gathered}
$$

The $4^{\text {th }}$ constitutive assumption in rate form can be written:

$$
\operatorname{tr}\left(\dot{\mathbf{G}}_{\mathrm{t}}\right)=0
$$

The constitutive relation for the evolution of $\underline{\mathbf{G}}_{\mathrm{t}}$ must be cast in an objective form by introducing an objective measure of the driving stress and the Jaumann co-rotational rate of $\underline{\mathbf{G}}_{t}$

$$
\underline{\mathbf{G}}_{\mathrm{t}}^{\nabla}=\underline{\dot{\mathbf{G}}}_{\mathrm{t}}-\underline{\boldsymbol{\omega}}_{\mathbf{G}} \underline{\mathbf{G}}_{\mathrm{t}}+\underline{\mathbf{G}}_{\mathrm{t}} \underline{\boldsymbol{\omega}}
$$

Where $\underline{\boldsymbol{\omega}}$ is a skew-symmetric structural spin tensor. Because the textural tensor is taken to deform in an affine manner with the inelastic deformation, the structural spin is equal to the spin of the unloaded configuration of the continuum:

$$
\underline{\omega}=\underline{\mathbf{W}}^{\mathrm{i}}
$$

As mentioned in $6.2, \underline{\mathbf{R}}^{\mathrm{i}}$ is assumed to equal $\underline{\mathbf{I}}$. Therefore:

$$
\underline{\mathbf{W}}^{\mathbf{i}}=\underline{\mathbf{R}}^{\mathbf{i}} \underline{\mathbf{R}}^{\mathbf{i}^{\mathbf{T}}}=\underline{\mathbf{0}}
$$

Thus, the structural spin vanishes, and the time derivative of the fabric ellipsoid is objective: 


$$
\underline{\mathbf{G}}_{\mathrm{t}}^{\nabla}=\underline{\dot{\mathbf{G}}}_{\mathrm{t}}
$$

Eq B.76

Therefore the inelastic velocity gradient is equal to the rate of deformation tensor:

$$
\underline{\mathbf{L}}^{\mathrm{i}}=\underline{\mathbf{D}}^{\mathrm{i}}
$$

Eq B.77

This allows Eq B.71 to be recast as:

$$
\underline{\mathbf{G}}_{\mathrm{t}}=\underline{\mathbf{D}}^{\mathrm{i}} \underline{\mathbf{G}}_{\mathrm{t}}+\underline{\mathbf{G}}_{\mathrm{t}} \underline{\mathbf{D}}^{\mathrm{i}}
$$

A flow rule is then cast in the form:

$$
\dot{\mathbf{G}}_{\mathrm{t}}=\dot{\gamma} \underline{\mathbf{N}}
$$

Where $\dot{\gamma}$ is a scalar measure of the magnitude of the rate of evolution, and $\underline{\mathbf{N}}$ is the deviatoric tensorial direction of the flow. $\dot{\gamma}$ scales with the magnitude of a driving stress. Using an approximate measure for $\underline{\mathbf{D}}^{\mathrm{i}}$ :

$$
\underline{\mathbf{D}}_{\mathrm{approx}}^{\mathrm{i}}=\frac{1}{4}\left(\underline{\dot{\mathbf{G}}}_{\mathrm{t}} \underline{\mathbf{G}}_{\mathrm{t}}^{-1}+\underline{\mathbf{G}}_{\mathrm{t}}^{-1} \dot{\mathbf{G}}_{\mathrm{t}}\right)
$$

And, imposing the constraint of Eq B.58:

$$
\underline{\underline{\Xi}} \underline{\mathbf{G}}_{\mathrm{t}}^{-1}+\underline{\mathbf{G}}_{\mathrm{t}}^{-1} \underline{\boldsymbol{E}} \geq 0
$$

The stress measure driving the evolution of the texture tensor is then expressed as:

$$
\underline{\mathbf{T}}_{\text {flow }}=\frac{1}{2}\left(\underline{\boldsymbol{\Xi}} \underline{\mathbf{G}}_{\mathrm{t}}^{-1}+\underline{\mathbf{G}}_{\mathrm{t}}^{-1} \boldsymbol{\Xi}\right)
$$

The direction of the flow is:

$$
\underline{\mathbf{N}}=\frac{\operatorname{dev}\left(\underline{\mathbf{T}}_{\text {flow }}\right)}{\left\|\operatorname{dev}\left(\underline{\mathbf{T}}_{\text {flow }}\right)\right\|}
$$


Where $\|\underline{\mathbf{A}}\|=\sqrt{\underline{\mathbf{A}}: \underline{\mathbf{A}}}$, and $\operatorname{dev}(\underline{\mathbf{A}})=\underline{\mathbf{A}}-\frac{1}{3} \operatorname{tr}(\underline{\mathbf{A}})$.

The measure for the magnitude of the driving stress is:

$$
\tau=\frac{\left\|\operatorname{dev}\left(\underline{\mathbf{T}}_{\text {flow }}\right)\right\|}{\sqrt{2}}
$$

The constitutive relationship for the flow strain rate is:

$$
\begin{gathered}
\dot{\gamma}=\dot{\gamma}_{0}\left(\frac{\tau-\tau_{0}}{\mathrm{~S}_{\mathrm{t}}}\right)^{\mathrm{m}}\left(\operatorname{det}\left(\underline{\mathbf{G}}_{\mathrm{t}}\right)-v \operatorname{det}\left(\underline{\mathbf{G}}_{0}\right)\right) ;\left(\tau-\tau_{0}\right) \&\left(\operatorname{det}\left(\underline{\mathbf{G}}_{\mathrm{t}}\right)-v \operatorname{det}\left(\underline{\mathbf{G}}_{0}\right)\right)>0 \\
\dot{\gamma}=0 ;\left(\tau-\tau_{0}\right) \leq 0 \text { or }\left\{\operatorname{det}(\underline{\mathbf{N}})<0 \&\left(\operatorname{det}\left(\underline{\mathbf{G}}_{\mathrm{t}}\right)-v \operatorname{det}\left(\underline{\mathbf{G}}_{0}\right)\right)=0\right\}
\end{gathered}
$$

The term $\left(\frac{\tau-\tau_{0}}{\mathrm{~S}_{\mathrm{t}}}\right)^{\mathrm{m}}$ is meant to capture the nonlinear dependence of the rate of structural evolution on the magnitude of the driving stress. This term relates the contribution to the resistance to texture evolution arising from interfiber friction. The parameter $\tau_{0}$ represents the initial threshold of resistance to textural evolution due to static friction. $\mathrm{S}_{\mathrm{t}}$ represents the material flow strength at time $t$ and evolves according to the equation:

$$
\mathrm{S}_{\mathrm{t}}=\mathrm{S}_{0}+\int \alpha \dot{\gamma} \mathrm{dt}
$$

The term $\left(\operatorname{det}\left(\underline{\mathbf{G}}_{t}\right)-v \operatorname{det}\left(\underline{\mathbf{G}}_{0}\right)\right)$ is meant to capture volume exclusion, wherein structural evolution ceases when a limiting (high) volume fraction is achieved.

The constitutive model is recast in a corotational framework and appended with a damage evolution law as described in 6.2.2. 
An additional constraint imposed in the new formulation of the JS model is the cessation of structural evolution and damage growth under compressive hydrostatic stress. This is expressed as:

$$
\dot{\gamma}=0 \& \dot{\mathrm{d}}=0 ; \sigma_{\mathrm{h}}=\frac{1}{3} \operatorname{tr}(\underline{\boldsymbol{\sigma}}) \leq 0
$$

In practice, some permanent structural evolution does take place during compaction, but, in Fiberloft, seems to be a very small effect. This constraint could be altered to allow some amount of structural evolution by defining a limiting hydrostatic stress, after which inelastic deformation ceases.

The update algorithm for this constitutive model is summarized below:

- $\quad$ Read in: $\underline{\mathbf{U}}_{\mathrm{t}+\mathrm{dt}}, \mathrm{dt}, \underline{\mathbf{G}}_{\mathrm{t}_{\mathrm{t}}}, \underline{\mathbf{U}}_{\mathrm{t}}^{\mathrm{i}}, \dot{\mathrm{G}}_{\mathrm{t}_{\mathrm{t}}}, \mathrm{d}, \mathrm{d}^{\mathrm{v}}$

- Update the structural tensor: $\underline{\mathbf{G}}_{\mathrm{t}_{\mathrm{t}+\mathrm{dt}}}=\underline{\mathbf{G}}_{\mathrm{t}_{\mathrm{t}}}+\underline{\mathbf{G}}_{\mathrm{t}_{\mathrm{t}}}$

- Solve for the inelastic rate of deformation tensor using

- Calculate the inelastic stretch from: $\underline{\mathbf{U}}_{t+\mathrm{dt}}^{\mathrm{i}}=\underline{\mathbf{U}}_{t}^{\mathrm{i}}+\underline{\mathbf{D}}^{\mathrm{i}} \underline{\mathbf{U}}_{t}^{\mathrm{i}} \mathrm{dt}$

- Calculate the elastic stretch from: $\underline{\mathbf{U}}^{\mathrm{e}}=\underline{\mathbf{U}}_{\mathrm{t}+\mathrm{dt}} \underline{\mathbf{U}}_{\mathrm{t}+\mathrm{dt}}^{\mathrm{i}^{-1}}$

- Determine the damage affected Cauchy stress from Eq 6.18

- Calculate the flow stress using Eq B.59 for the Mandel Stress (weighted by the factor (1d) to account for damage effects) and Eq B.82 for the flow stress

- Calculate the direction and magnitude of the inelastic flow from Eq B.83 and Eq B.84

- Calculate the structural flow rate from Eq B.79

- Calculate $\mathrm{S}_{\mathrm{t}}$ from Eq B.87

- $\quad$ Update the damage parameter using the damage model discussed in 6.2.1

- Save the state variables for the next time increment 


\section{Appendix C. JS VUMAT FORTRAN Code}

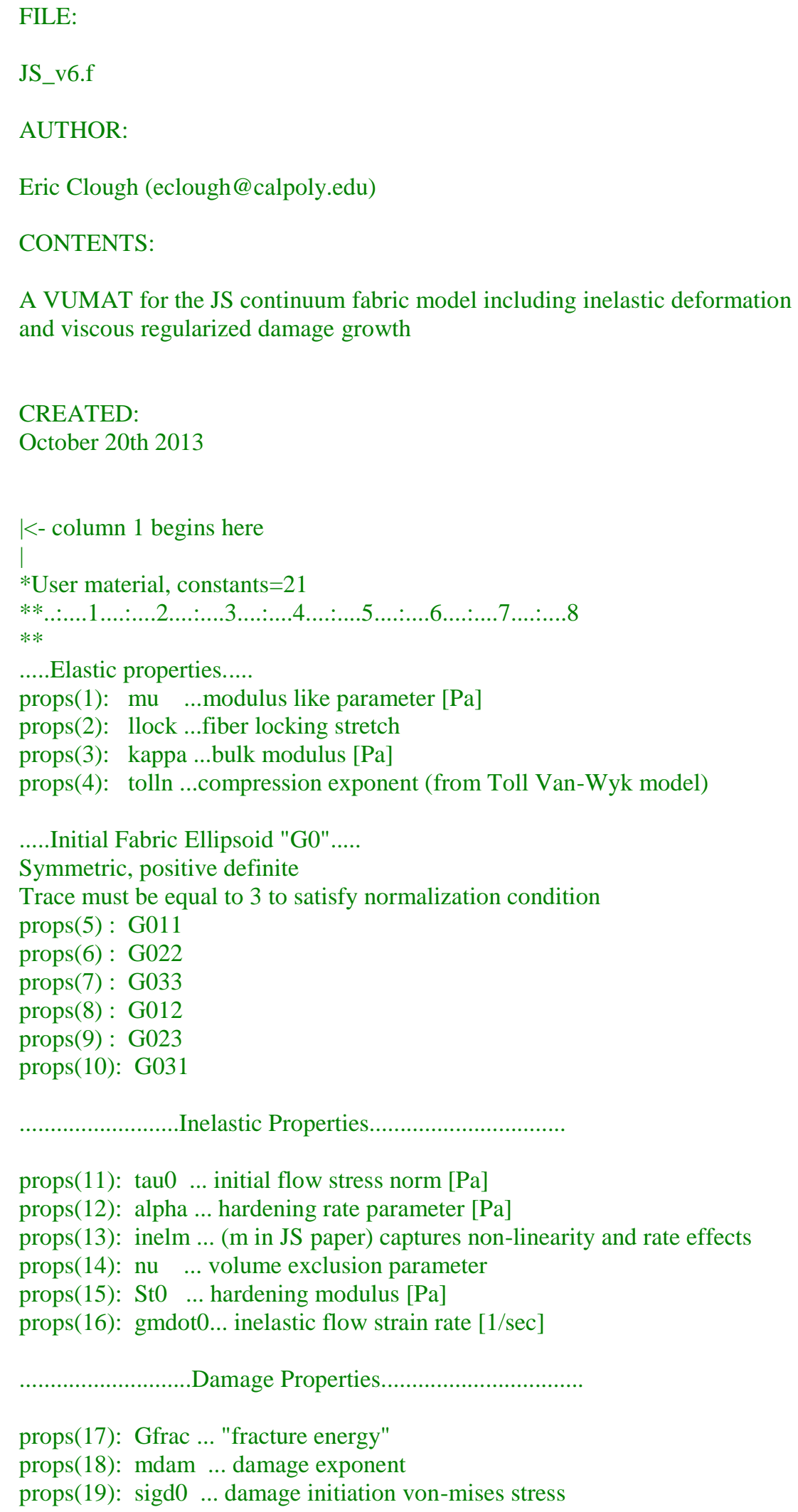




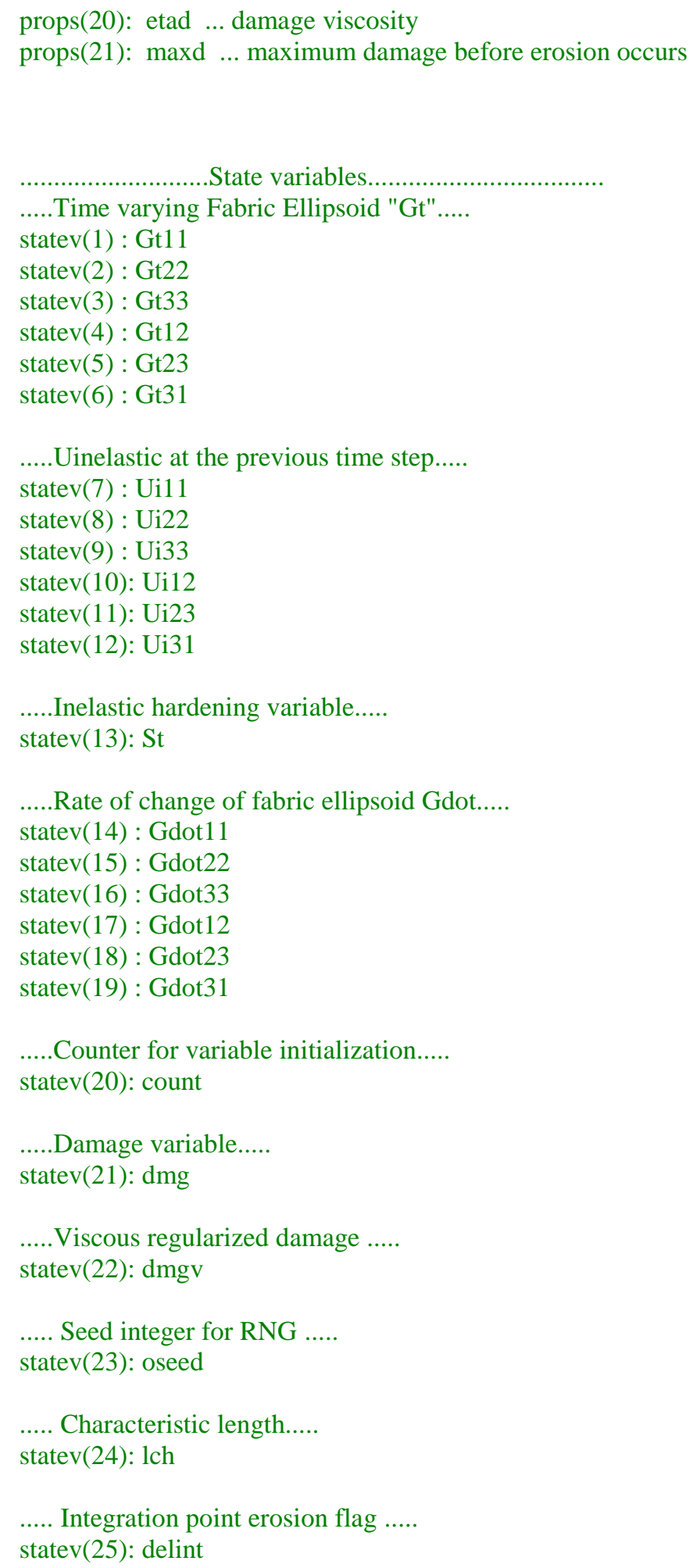


! the parameter "zero." This may not be necessary depending on the problem.

! SUBROUTINES

1. push $\rightarrow$ tensor push forward $\mathrm{U}^{*} \mathrm{~A}^{*} \mathrm{U}^{\wedge} \mathrm{T}$

! 2. Inginv $\rightarrow$ Calculates the inverse Langevin function

! 3. luinv $\rightarrow$ Inverts an $\mathrm{n} \times \mathrm{n}$ matrix using an $\mathrm{LU}$ decomposition

! 4. ludcmp -> performs an LU decomposition of a matrix

! 5. lubksb -> solves a system of equations with an LU decomposed LHS

! 6. norm $\rightarrow$ calculates the norm of a symmetric $3 \times 3$ tensor

! 7. srand -> pseudorandom number generator

Start Abaqus required VUMAT Header

1

subroutine vumat (

! Read only (unmodifiable)variables -

1 nblock, ndir, nshr, nstatev, nfieldv, nprops, lanneal,

stepTime, totalTime, dt, cmname, coordMp, charLength,

props, density, strainInc, relSpinInc,

tempOld, stretchOld, defgradOld, fieldOld,

stressOld, stateOld, enerInternOld, enerInelasOld,

tempNew, stretchNew, defgradNew, fieldNew,

! Write only (modifiable) variables -

7 stressNew, stateNew, enerInternNew, enerInelasNew )

!

include 'vaba_param.inc'

dimension props(nprops), density(nblock), coordMp(nblock, ${ }^{*}$ ),

charLength(nblock), strainInc(nblock,ndir+nshr),

relSpinInc(nblock,nshr), tempOld(nblock),

stretchOld(nblock,ndir+nshr),

defgradOld(nblock,ndir+nshr+nshr),

fieldOld(nblock,nfieldv), stressOld(nblock,ndir+nshr),

stateOld(nblock,nstatev), enerInternOld(nblock),

enerInelasOld(nblock), tempNew(nblock),

stretchNew(nblock,ndir+nshr),

defgradNew(nblock,ndir+nshr+nshr),

fieldNew(nblock,nfieldv),

stressNew(nblock,ndir+nshr), stateNew(nblock,nstatev),

enerInternNew(nblock), enerInelasNew(nblock)

!

character $* 80$ cmname

* $\quad$ End VUMAT required Header

! define variables

real $\mathrm{U}(3,3), \mathrm{G} 0(3,3)$, pushGt $(3,3)$, trG0, leff,llock,Jac,t1,t2,t3,t4, mu,kappa,tolln,beta,beta0,Ui(3,3),Ue(3,3), $\operatorname{Di}(3,3), \mathrm{A}(3,3)$,

$\operatorname{trGt}, \operatorname{SPK}(3,3), \mathrm{Xi}(3,3), \mathrm{Je}, \mathrm{Ceinv}(3,3), \mathrm{N}(3,3)$,tau0,alpha,inelm, nu,St0,gmdot0, detGt,detG0,Gt(3,3),Gtinv(3,3), Gdot(3,3),tau, gamdot,St,Tflow(3,3),dmg,Gfrac,lch,rand,dmgv,maxd,seff,sigd0, admg,dsr

integer $\mathrm{i}, \mathrm{j}, \mathrm{k}$, count,oseed

parameter zero $=0 \mathrm{~d} 0$, one $=1 \mathrm{~d} 0$, two $=2 \mathrm{~d} 0$, half $=5 \mathrm{~d}-1$, third $=1 \mathrm{~d} 0 / 3 \mathrm{~d} 0$, 
2 fourth $=1 \mathrm{~d} 0 / 4 \mathrm{~d} 0$, tiny $=1 \mathrm{e}-32$, twothird $=2 \mathrm{~d} 0 / 3 \mathrm{~d} 0$, threehalf $=3 \mathrm{~d} 0 / 2 \mathrm{~d} 0$

! Elastic Properties

$\mathrm{mu}=\operatorname{props}(1)$

llock $=\operatorname{props}(2)$

kappa $=\operatorname{props}(3)$

tolln $=\operatorname{props}(4)$

! $\quad$ setup G0 (fabric ellipsoid in ref config)

$\mathrm{G} 0(1,1)=\operatorname{props}(5)$

$\mathrm{G} 0(2,2)=\operatorname{props}(6)$

$\mathrm{G} 0(3,3)=\operatorname{props}(7)$

$\mathrm{G} 0(1,2)=\operatorname{props}(8)$

$\mathrm{G} 0(2,3)=\operatorname{props}(9)$

$\mathrm{G} 0(3,1)=\operatorname{props}(10)$

$\mathrm{G} 0(2,1)=\operatorname{props}(8)$

$\mathrm{G} 0(3,2)=\operatorname{props}(9)$

$\mathrm{G} 0(1,3)=\operatorname{props}(10)$

! Inelastic Properties

tau $0=\operatorname{props}(11)$

alpha $=\operatorname{props}(12)$

inelm $=\operatorname{props}(13)$

$\mathrm{nu}=\operatorname{props}(14)$

$\mathrm{St} 0=\operatorname{props}(15)$

gmdot $0=\operatorname{props}(16)$

! Damage Properties

Gfrac $=\operatorname{props}(17)$

mdam $=\operatorname{props}(18)$

$\operatorname{sigd} 0=\operatorname{props}(19)$

etad $=\operatorname{props}(20)$

$\operatorname{maxd}=\operatorname{props}(21)$

! Calculate the trace of G0

! this should always equal 3

$\operatorname{trG} 0=\mathrm{G} 0(1,1)+\mathrm{G} 0(2,2)+\mathrm{G} 0(3,3)$

! Calculate the determinant of G0

$\mathrm{t} 1=\mathrm{G} 0(1,1) *(\mathrm{G} 0(2,2) * \mathrm{G} 0(3,3)-\mathrm{G} 0(2,3) * * 2)$

$\mathrm{t} 2=\mathrm{G} 0(1,2) *(\mathrm{G} 0(2,3) * \mathrm{G} 0(1,3)-\mathrm{G} 0(1,2) * \mathrm{G} 0(3,3))$

$\mathrm{t} 3=\mathrm{G} 0(1,3) *(\mathrm{G} 0(1,2) * \mathrm{G} 0(2,3)-\mathrm{G} 0(2,2) * \mathrm{G} 0(1,3))$

$\operatorname{det} \mathrm{G} 0=\mathrm{t} 1+\mathrm{t} 2+\mathrm{t} 3$

! calculate beta0, which will be the same for all material points

leff=one !effective stretch in the reference configuration

call lnginv(leff,llock,beta0) ! gets beta0

IF (stepTime.eq.0) then !1

! forces the material to behave elastically for the data check increment

Do i $=1$, nblock

$\mathrm{Ue}(1,1)=\operatorname{stretchNew}(\mathrm{i}, 1)$

$\mathrm{Ue}(2,2)=\operatorname{stretchNew}(\mathrm{i}, 2)$

$\mathrm{Ue}(3,3)=\operatorname{stretchNew}(\mathrm{i}, 3)$

$\mathrm{Ue}(1,2)=\operatorname{stretchNew}(\mathrm{i}, 4)$ 
$\mathrm{Ue}(2,1)=\mathrm{Ue}(1,2)$

$\mathrm{Ue}(2,3)=\operatorname{stretchNew}(\mathrm{i}, 5)$

$\mathrm{Ue}(3,2)=\mathrm{Ue}(2,3)$

$\mathrm{Ue}(1,3)=\operatorname{stretchNew}(\mathrm{i}, 6)$

$\mathrm{Ue}(3,1)=\mathrm{Ue}(1,3)$

! Calculate the Jacobian of Ue

$\mathrm{t} 1=\mathrm{Ue}(1,1) *(\mathrm{Ue}(2,2) * \mathrm{Ue}(3,3)-\mathrm{Ue}(2,3) * * 2)$

$\mathrm{t} 2=\mathrm{Ue}(1,2) *(\mathrm{Ue}(2,3) * \mathrm{Ue}(1,3)-\mathrm{Ue}(1,2) * \mathrm{Ue}(3,3))$

$\mathrm{t} 3=\mathrm{Ue}(1,3) *(\mathrm{Ue}(1,2) * \mathrm{Ue}(2,3)-\mathrm{Ue}(2,2) * \mathrm{Ue}(1,3))$

$\mathrm{Je}=\mathrm{t} 1+\mathrm{t} 2+\mathrm{t} 3$

$\mathrm{Gt}=\mathrm{G} 0$

! Calculate the push forward of Gt

call push(Gt,Ue,ndir,pushGt)

leff=sqrt $((\operatorname{pushGt}(1,1)+\operatorname{pushGt}(2,2)+\operatorname{pushGt}(3,3)) / \operatorname{trG} 0)$

call lnginv(leff,llock,beta)! gets beta

$\mathrm{t} 1=\mathrm{mu} /(\mathrm{Je} * \mathrm{trG} 0)$ !leading coefficient

$\mathrm{t} 2=$ llock/leff $*$ beta $!$ inverse of the stretch ratio $*$ beta

t3 $=$ kappa* $\left(\left(\mathrm{Je}^{* *}(-\right.\right.$ tolln $\left.\left.)\right)-1\right)$ !Toll Van Wyk term

$\mathrm{t} 4=$ llock $*$ beta 0

! Update the Cauchy stress

stressNew(i,1) $=\mathrm{t} 1 *(\mathrm{t} 2 * \operatorname{pushGt}(1,1)-\mathrm{t} 4 * \mathrm{Gt}(1,1))$-t 3 !Sig1 1

stressNew $(\mathrm{i}, 2)=\mathrm{t} 1 *(\mathrm{t} 2 * \operatorname{pushGt}(2,2)-\mathrm{t} 4 * \mathrm{Gt}(2,2))-\mathrm{t} 3$ !Sig22

stressNew $(\mathrm{i}, 3)=\mathrm{t} 1 *(\mathrm{t} 2 * \operatorname{pushGt}(3,3)-\mathrm{t} 4 * \mathrm{Gt}(3,3))-\mathrm{t} 3$ !Sig33

stressNew $(\mathrm{i}, 4)=\mathrm{t} 1 *(\mathrm{t} 2 * \operatorname{pushGt}(1,2)-\mathrm{t} 4 * \mathrm{Gt}(1,2)) \quad$ !Sig12

stressNew $(\mathrm{i}, 5)=\mathrm{t} 1 *(\mathrm{t} 2 * \operatorname{pushGt}(2,3)-\mathrm{t} 4 * \mathrm{Gt}(2,3)) \quad$ !Sig23

stressNew $(\mathrm{i}, 6)=\mathrm{t} 1 *(\mathrm{t} 2 * \operatorname{pushGt}(3,1)-\mathrm{t} 4 * \mathrm{Gt}(3,1)) \quad$ !Sig31

! ..... Store the new Gt

stateNew $(\mathrm{i}, 1)=\mathrm{Gt}(1,1)$

stateNew $(\mathrm{i}, 2)=\mathrm{Gt}(2,2)$

stateNew $(\mathrm{i}, 3)=\mathrm{Gt}(3,3)$

stateNew $(\mathrm{i}, 4)=\mathrm{Gt}(1,2)$

stateNew $(\mathrm{i}, 5)=\mathrm{Gt}(2,3)$

stateNew $(\mathrm{i}, 6)=\mathrm{Gt}(3,1)$

$1 \quad$..... Store the new Uinelastic .....

stateNew $(i, 7)=$ one

stateNew $(\mathrm{i}, 8)=$ one

stateNew $(\mathrm{i}, 9)=$ one

stateNew $(\mathrm{i}, 10)=$ zero

stateNew $(i, 11)=$ zero

stateNew $(i, 12)=$ zero

! Make sure that erosion is off for the data check increment stateNew $(\mathrm{i}, 25)=$ one

enddo

ELSE

Loop through all material points 
do $100 \mathrm{i}=1$, nblock

$*$

* Initialize the state variables

! update count

count=stateOld $(\mathrm{i}, 20)+1$

!if in the first increment initialize variables

if (count.le.1) then !2

! Set Gt to G0

$\mathrm{Gt}=\mathrm{G} 0$

! Set Ui to the identity tensor

$\mathrm{Ui}(1,1)=$ one

$\mathrm{Ui}(2,2)=$ one

$\mathrm{Ui}(3,3)=$ one

$\mathrm{Ui}(1,2)=$ zero

$\mathrm{Ui}(2,3)=$ zero

$\mathrm{Ui}(3,1)=$ zero

$\mathrm{Ui}(2,1)=$ zero

$\mathrm{Ui}(3,2)=$ zero

$\mathrm{Ui}(1,3)=$ zero

! Set Gdot to zeros

$\operatorname{Gdot}(1,1)=$ zero

$\operatorname{Gdot}(2,2)=$ zero

$\operatorname{Gdot}(3,3)=$ zero

$\operatorname{Gdot}(1,2)=$ zero

$\operatorname{Gdot}(2,3)=$ zero

$\operatorname{Gdot}(3,1)=$ zero

$\operatorname{Gdot}(2,1)=$ zero

$\operatorname{Gdot}(3,2)=$ zero

$\operatorname{Gdot}(1,3)=$ zero

! initialize the damage variable

$\mathrm{dmg}=$ zero

dmgv=zero

! initialize the characteristic length

stateNew $(\mathrm{i}, 24)=$ charLength $(\mathrm{i})$

! intialize erosion flag

stateNew $(\mathrm{i}, 25)=$ one

! Set St to St0

$\mathrm{St}=\mathrm{St} 0 ! \mathrm{St}$

else !setup from stateOld

! Setup Gt

$\operatorname{Gt}(1,1)=$ stateOld $(\mathrm{i}, 1)$

$\mathrm{Gt}(2,2)=$ stateOld $(\mathrm{i}, 2)$

$\operatorname{Gt}(3,3)=$ stateOld $(\mathrm{i}, 3)$

$\operatorname{Gt}(1,2)=$ stateOld $(\mathrm{i}, 4)$

$\operatorname{Gt}(2,3)=\operatorname{stateOld}(\mathrm{i}, 5)$

$\mathrm{Gt}(3,1)=$ stateOld $(\mathrm{i}, 6)$

$\mathrm{Gt}(2,1)=$ stateOld $(\mathrm{i}, 4)$

$\operatorname{Gt}(3,2)=$ stateOld $(\mathrm{i}, 5)$ 
$\operatorname{Gt}(1,3)=\operatorname{stateOld}(\mathrm{i}, 6)$

! Setup the old inelastic stretch

$\mathrm{Ui}(1,1)=\operatorname{stateOld}(\mathrm{i}, 7)$

$\mathrm{Ui}(2,2)=$ stateOld $(\mathrm{i}, 8)$

$\mathrm{Ui}(3,3)=$ stateOld $(\mathrm{i}, 9)$

$\mathrm{Ui}(1,2)=\operatorname{stateOld}(\mathrm{i}, 10)$

$\mathrm{Ui}(2,3)=$ stateOld $(\mathrm{i}, 11)$

$\mathrm{Ui}(3,1)=\operatorname{stateOld}(\mathrm{i}, 12)$

$\mathrm{Ui}(2,1)=\mathrm{Ui}(1,2)$

$\mathrm{Ui}(3,2)=\mathrm{Ui}(2,3)$

$\mathrm{Ui}(1,3)=\mathrm{Ui}(3,1)$

! Get the old St (hardening parameter)

$\mathrm{St}=$ stateOld$(\mathrm{i}, 13)$

! Setup Gdot

$\operatorname{Gdot}(1,1)=$ stateOld $(\mathrm{i}, 14)$

$\operatorname{Gdot}(2,2)=\operatorname{stateOld}(\mathrm{i}, 15)$

$\operatorname{Gdot}(3,3)=$ stateOld $(\mathrm{i}, 16)$

$\operatorname{Gdot}(1,2)=\operatorname{stateOld}(i, 17)$

$\operatorname{Gdot}(2,3)=\operatorname{stateOld}(\mathrm{i}, 18)$

$\operatorname{Gdot}(3,1)=\operatorname{stateOld}(i, 19)$

$\operatorname{Gdot}(2,1)=\operatorname{stateOld}(\mathrm{i}, 17)$

$\operatorname{Gdot}(3,2)=\operatorname{stateOld}(\mathrm{i}, 18)$

$\operatorname{Gdot}(1,3)=\operatorname{stateOld}(\mathrm{i}, 19)$

! damage variable

$\mathrm{dmg}=\operatorname{stateOld}(\mathrm{i}, 21)$

! viscous regularized damage variable

dmgv=stateOld $(\mathrm{i}, 22)$

! Integer seed for RNG

oseed=stateOld $(\mathrm{i}, 23)$

! ! store the characteristic length

lch $=$ CharLength(i)

stateNew $(\mathrm{i}, 24)=\mathrm{lch}$

! integration point erosion flag

stateNew $(\mathrm{i}, 25)=\operatorname{stateOld}(\mathrm{i}, 25)$

endif $! 2$

*

* Update U, Gt, Di, Ui, Ue, pushGt, leff, and Beta setup U

This model will be formulated in a corotational reference

frame so we will use $\mathrm{U}$ rather than $\mathrm{F}$

$\mathrm{U}(1,1)=\operatorname{stretchNew}(\mathrm{i}, 1)$

$\mathrm{U}(2,2)=\operatorname{stretchNew}(\mathrm{i}, 2)$

$\mathrm{U}(3,3)=\operatorname{stretchNew}(\mathrm{i}, 3)$

$\mathrm{U}(1,2)=\operatorname{stretchNew}(\mathrm{i}, 4)$

$\mathrm{U}(2,3)=\operatorname{stretchNew}(\mathrm{i}, 5)$

$\mathrm{U}(1,3)=\operatorname{stretchNew}(\mathrm{i}, 6)$

$\mathrm{U}(3,2)=\mathrm{U}(2,3)$ 
$\mathrm{U}(2,1)=\mathrm{U}(1,2)$

$\mathrm{U}(3,1)=\mathrm{U}(1,3)$

! calculate the Jacobian of $\mathrm{U}$

$\mathrm{t} 1=\mathrm{U}(1,1) *(\mathrm{U}(2,2) * \mathrm{U}(3,3)-\mathrm{U}(2,3) * * 2)$

$\mathrm{t} 2=\mathrm{U}(1,2) *(\mathrm{U}(2,3) * \mathrm{U}(1,3)-\mathrm{U}(1,2) * \mathrm{U}(3,3))$

$\mathrm{t} 3=\mathrm{U}(1,3) *(\mathrm{U}(1,2) * \mathrm{U}(2,3)-\mathrm{U}(2,2) * \mathrm{U}(1,3))$

$\mathrm{Jac}=\mathrm{t} 1+\mathrm{t} 2+\mathrm{t} 3$

! Update Gt using gamdot from the previous increment

$\mathrm{Gt}=\mathrm{Gt}+\mathrm{Gdot}^{*} \mathrm{dt}$

do $\mathrm{j}=1$, ndir

do $\mathrm{k}=1$, ndir

if $(\mathrm{Gt}(\mathrm{j}, \mathrm{k}) \cdot \mathrm{lt} \cdot \mathrm{zero})$ then

$\mathrm{Gt}(\mathrm{j}, \mathrm{k})=\mathrm{zero}$

endif

if $(\operatorname{abs}(\mathrm{Gt}(\mathrm{j}, \mathrm{k})) \cdot$.le.tiny $)$ then

$\mathrm{Gt}(\mathrm{j}, \mathrm{k})=\mathrm{zero}$

endif

enddo

enddo

! Calculate the trace of Gt

$\operatorname{trGt}=\mathrm{Gt}(1,1)+\mathrm{Gt}(2,2)+\mathrm{Gt}(3,3)$

! Calculate the determinant of $\mathrm{Gt}$

$\mathrm{t} 1=\mathrm{Gt}(1,1) *(\mathrm{Gt}(2,2) * \mathrm{Gt}(3,3)-\mathrm{Gt}(2,3) * * 2)$

$\mathrm{t} 2=\mathrm{Gt}(1,2) *(\mathrm{Gt}(2,3) * \mathrm{Gt}(1,3)-\mathrm{Gt}(1,2) * \mathrm{Gt}(3,3))$

$\mathrm{t} 3=\mathrm{Gt}(1,3) *(\mathrm{Gt}(1,2) * \mathrm{Gt}(2,3)-\mathrm{Gt}(2,2) * \mathrm{Gt}(1,3))$

$\operatorname{det} \mathrm{t}=\mathrm{t} 1+\mathrm{t} 2+\mathrm{t} 3$

! Solve for Di using the approximate eqn $\mathrm{Di}=1 / 4 *(\mathrm{inv}(\mathrm{Gt}) * \mathrm{Gdot}+\mathrm{Gdot} * \mathrm{inv}(\mathrm{Gt}))$ call diup(Gt,Gdot,ndir,Di,Gtinv)

! Update the inelastic stretch

$\mathrm{Ui}=\mathrm{Ui}+\operatorname{matmul}(\mathrm{Di}, \mathrm{Ui}) * \mathrm{dt}$

! Calculate $\mathrm{Ue}$ from $\mathrm{Ue}=\mathrm{U}^{*} \mathrm{inv}(\mathrm{Ui}), \mathrm{A}=\mathrm{inv}(\mathrm{Ui})$ in this case

call luinv(Ui,ndir,A)

$\mathrm{Ue}=\operatorname{matmul}(\mathrm{U}, \mathrm{A})$

! Calculate the Jacobian of Ue

$\mathrm{t} 1=\mathrm{Ue}(1,1) *(\mathrm{Ue}(2,2) * \mathrm{Ue}(3,3)-\mathrm{Ue}(2,3) * * 2)$

$\mathrm{t} 2=\mathrm{Ue}(1,2) *(\mathrm{Ue}(2,3) * \mathrm{Ue}(1,3)-\mathrm{Ue}(1,2) * \mathrm{Ue}(3,3))$

$\mathrm{t} 3=\mathrm{Ue}(1,3) *(\mathrm{Ue}(1,2) * \mathrm{Ue}(2,3)-\mathrm{Ue}(2,2) * \mathrm{Ue}(1,3))$

$\mathrm{Je}=\mathrm{t} 1+\mathrm{t} 2+\mathrm{t} 3$

! Calculate the push forward of Gt

call push(Gt,Ue,ndir,pushGt)

! gets pushGt

! Calculate the effective elastic stretch

! leff $=\operatorname{sqrt}\left(\operatorname{tr}\left(\mathrm{U}^{*} \mathrm{Gt} * \mathrm{U}\right) / \operatorname{tr}(\mathrm{Gt})\right)$

leff=sqrt((pushGt $(1,1)+\operatorname{pushGt}(2,2)+\operatorname{pushGt}(3,3)) / \operatorname{trGt})$

! Calculate the inverse Langevin function of the stretch ratio

call lnginv(leff,llock,beta) 
! gets beta

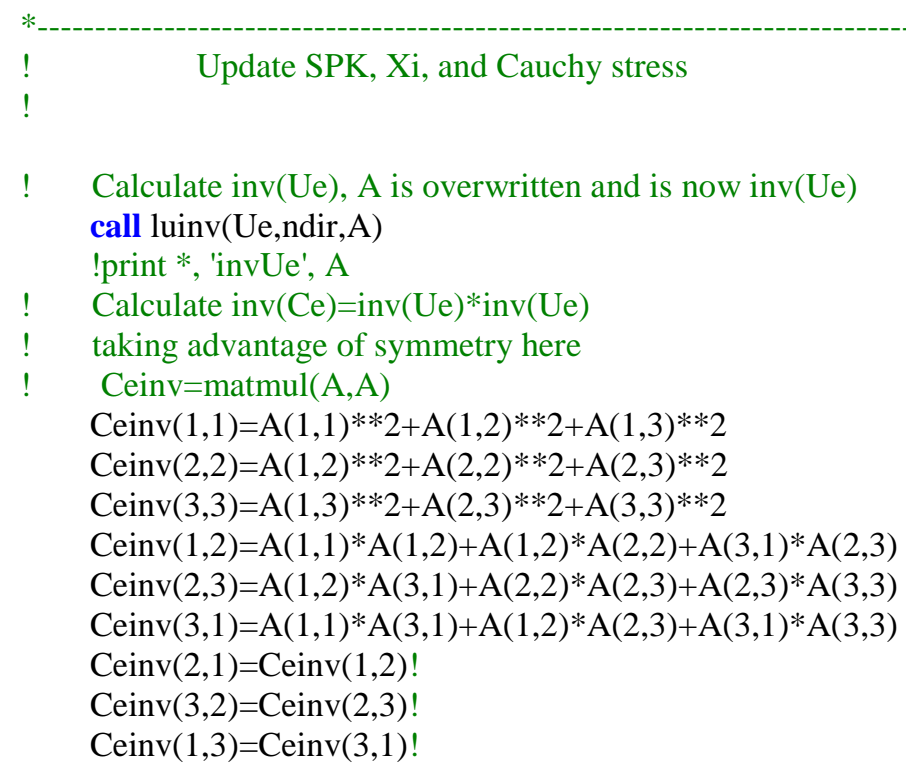

$*$

!

$!$

Update SPK, Xi, and Cauchy stress

! Calculate $\operatorname{inv}(\mathrm{Ue}), \mathrm{A}$ is overwritten and is now $\operatorname{inv}(\mathrm{Ue})$

call luinv(Ue,ndir,A)

!print *, 'invUe', A

! Calculate $\operatorname{inv}(\mathrm{Ce})=\operatorname{inv}(\mathrm{Ue}) * \operatorname{inv}(\mathrm{Ue})$

! taking advantage of symmetry here

! $\quad$ Ceinv $=$ matmul $(\mathrm{A}, \mathrm{A})$

$\operatorname{Ceinv}(1,1)=\mathrm{A}(1,1) * * 2+\mathrm{A}(1,2) * * 2+\mathrm{A}(1,3) * * 2$

$\operatorname{Ceinv}(2,2)=\mathrm{A}(1,2) * * 2+\mathrm{A}(2,2) * * 2+\mathrm{A}(2,3) * * 2$

$\operatorname{Ceinv}(3,3)=\mathrm{A}(1,3) * * 2+\mathrm{A}(2,3) * * 2+\mathrm{A}(3,3) * * 2$

$\operatorname{Ceinv}(1,2)=\mathrm{A}(1,1) * \mathrm{~A}(1,2)+\mathrm{A}(1,2) * \mathrm{~A}(2,2)+\mathrm{A}(3,1) * \mathrm{~A}(2,3)$

$\operatorname{Ceinv}(2,3)=\mathrm{A}(1,2) * \mathrm{~A}(3,1)+\mathrm{A}(2,2) * \mathrm{~A}(2,3)+\mathrm{A}(2,3) * \mathrm{~A}(3,3)$

$\operatorname{Ceinv}(3,1)=\mathrm{A}(1,1) * \mathrm{~A}(3,1)+\mathrm{A}(1,2) * \mathrm{~A}(2,3)+\mathrm{A}(3,1) * \mathrm{~A}(3,3)$

$\operatorname{Ceinv}(2,1)=\operatorname{Ceinv}(1,2)$ !

$\operatorname{Ceinv}(3,2)=\operatorname{Ceinv}(2,3)$ !

$\operatorname{Ceinv}(1,3)=\operatorname{Ceinv}(3,1)$ !

! SPK $=\mathrm{mu} /(\operatorname{tr}(\mathrm{Gt})) *(1$ lock/leff*beta*Gt-llock*beta $0 * \operatorname{inv}(\mathrm{Ue}) \mathrm{Gt} * \mathrm{inv}(\mathrm{Ue}))$ ! -kappa*Je*(Je^(-n)-1)*inv(Ce)

! this breaks down to: $\mathrm{Spk}=$ stretch term - repulsive term - bulk term the ! strain energy is scaled back by the damage to account for fiber slip from ! junction points

! overwrite $\mathrm{t} 1, \mathrm{t} 2, \mathrm{t} 3, \mathrm{t} 4$

$\mathrm{t} 1=\mathrm{mu} /(\mathrm{trGt}) *($ one-dmgv) !leading coefficient including damage

t $2=$ llock/leff $*$ beta !inverse of the stretch ratio times beta

t3 $=$ kappa*Je*((Je**(-tolln))-one)*(one-dmgv)!Toll Van Wyk term

$\mathrm{t} 4=$ llock $*$ beta 0

! overwrite $\mathrm{Xi}$ with the term: $\operatorname{inv}(\mathrm{Ue}) * \mathrm{Gt} * \operatorname{inv}(\mathrm{Ue})$

! this is actually the repulsive term in this context but will be overwritten

! later with the real $\mathrm{Xi}$

call push(Gt,A,ndir,Xi)

! Calculate second Piola Kirchoff stress: SPK

! in this case $\mathrm{Xi}$ is the repulsive stress term

! I also multiply the terms by their respective coefficients in this step

! taking advantage of symmetry here to save some computation

$\operatorname{SPK}(1,1)=\mathrm{t} 1 *(\mathrm{t} 2 * \mathrm{Gt}(1,1)-\mathrm{t} 4 * \mathrm{Xi}(1,1))-\mathrm{t} 3 * \operatorname{Ceinv}(1,1)$

$\operatorname{SPK}(2,2)=\mathrm{t} 1 *(\mathrm{t} 2 * \mathrm{Gt}(2,2)-\mathrm{t} 4 * \mathrm{Xi}(2,2))-\mathrm{t} 3 * \operatorname{Ceinv}(2,2)$

$\operatorname{SPK}(3,3)=\mathrm{t} 1 *(\mathrm{t} 2 * \mathrm{Gt}(3,3)-\mathrm{t} 4 * \mathrm{Xi}(3,3))-\mathrm{t} 3 * \operatorname{Ceinv}(3,3)$

$\operatorname{SPK}(1,2)=\mathrm{t} 1 *(\mathrm{t} 2 * \mathrm{Gt}(1,2)-\mathrm{t} 4 * \mathrm{Xi}(1,2))-\mathrm{t} 3 * \operatorname{Ceinv}(1,2)$

$\operatorname{SPK}(2,3)=\mathrm{t} 1 *(\mathrm{t} 2 * \mathrm{Gt}(2,3)-\mathrm{t} 4 * \mathrm{Xi}(2,3))-\mathrm{t} 3 * \operatorname{Ceinv}(2,3)$

$\operatorname{SPK}(3,1)=\mathrm{t} 1 *(\mathrm{t} 2 * \operatorname{Gt}(3,1)-\mathrm{t} 4 * \mathrm{Xi}(3,1))-\mathrm{t} 3 * \operatorname{Ceinv}(3,1)$

$\operatorname{SPK}(2,1)=\operatorname{SPK}(1,2)$

$\operatorname{SPK}(3,2)=\operatorname{SPK}(2,3)$

$\operatorname{SPK}(1,3)=\operatorname{SPK}(3,1)$

! Update the cauchy stress

! Sig $=\mathrm{mu} /(\operatorname{Jac} * \operatorname{tr}(\mathrm{Gt}))^{*}($ llock/leff*beta*pushGt-llock*beta $0 * \mathrm{Gt})-\operatorname{kappa}^{*}\left(\operatorname{Jac}^{\wedge}(-\mathrm{n})-1\right) * \mathrm{I}$ 
! update $\mathrm{t} 1$, and $\mathrm{t} 3$ according to the cauchy stress coefficients

! I don't use a push forward of SPK here due to the way I've defined the modulus

$\mathrm{t} 1=\mathrm{t} 1 / \mathrm{Jac}$

$\mathrm{t} 3=\mathrm{t} 3 / \mathrm{Je}$

stressNew $(\mathrm{i}, 1)=\mathrm{t} 1 *(\mathrm{t} 2 * \operatorname{pushGt}(1,1)-\mathrm{t} 4 * \mathrm{Gt}(1,1))-\mathrm{t} 3$ !Sig1 1

$\operatorname{stressNew}(\mathrm{i}, 2)=\mathrm{t} 1 *(\mathrm{t} 2 * \operatorname{pushGt}(2,2)-\mathrm{t} 4 * \mathrm{Gt}(2,2))-\mathrm{t} 3$ !Sig22

stressNew $(\mathrm{i}, 3)=\mathrm{t} 1 *(\mathrm{t} 2 * \operatorname{pushGt}(3,3)-\mathrm{t} 4 * \mathrm{Gt}(3,3))-\mathrm{t} 3$ !Sig33

stressNew $(\mathrm{i}, 4)=\mathrm{t} 1 *(\mathrm{t} 2 *$ pushGt $(1,2)-\mathrm{t} 4 * \mathrm{Gt}(1,2)) \quad$ !Sig12

stressNew $(\mathrm{i}, 5)=\mathrm{t} 1 *(\mathrm{t} 2 *$ pushGt $(2,3)-\mathrm{t} 4 * \mathrm{Gt}(2,3)) \quad$ !Sig23

stressNew $(\mathrm{i}, 6)=\mathrm{t} 1 *(\mathrm{t} 2 * \operatorname{pushGt}(3,1)-\mathrm{t} 4 * \mathrm{Gt}(3,1)) \quad$ !Sig31

!print *, 'stressnew', stressnew(i,:)

! Calculate the Mandel stress $\mathrm{Xi}=\mathrm{Ue} * \mathrm{Ue} * \mathrm{Spk}$

! Overwrite $\mathrm{Xi}$ (which was used for the repulsive term above) with $\mathrm{Ce}$

! Taking advantage of symmetry

$\mathrm{Xi}(1,1)=\mathrm{Ue}(1,1) * * 2+\mathrm{Ue}(1,2) * * 2+\mathrm{Ue}(1,3) * * 2$

$\mathrm{Xi}(2,2)=\mathrm{Ue}(1,2) * * 2+\mathrm{Ue}(2,2) * * 2+\mathrm{Ue}(2,3) * * 2$

$\mathrm{Xi}(3,3)=\mathrm{Ue}(1,3) * * 2+\mathrm{Ue}(2,3) * * 2+\mathrm{Ue}(3,3) * * 2$

$\mathrm{Xi}(1,2)=\mathrm{Ue}(1,1) * \mathrm{Ue}(1,2)+\mathrm{Ue}(1,2) * \mathrm{Ue}(2,2)+\mathrm{Ue}(3,1) * \mathrm{Ue}(2,3)$

$\mathrm{Xi}(2,3)=\mathrm{Ue}(1,2) * \mathrm{Ue}(3,1)+\mathrm{Ue}(2,2) * \mathrm{Ue}(2,3)+\mathrm{Ue}(2,3) * \mathrm{Ue}(3,3)$

$\mathrm{Xi}(3,1)=\mathrm{Ue}(1,1) * \mathrm{Ue}(3,1)+\mathrm{Ue}(1,2) * \mathrm{Ue}(2,3)+\mathrm{Ue}(3,1) * \mathrm{Ue}(3,3)$

$\mathrm{Xi}(2,1)=\mathrm{Xi}(1,2)$

$\mathrm{Xi}(3,2)=\mathrm{Xi}(2,3)$

$\mathrm{Xi}(1,3)=\mathrm{Xi}(3,1)$

! Calculate the Mandel stress: Xi

$\mathrm{Xi}=$ matmul(Xi,SPK) !overwrite $\mathrm{Xi}$ again, now $=$ Mandel stress

! Calculate the inelastic flow stress, its magnitude, and direction

Tflow=half*(matmul(Xi,Gtinv) + matmul(Gtinv,Xi))

! Calculate the volumetric part of Tflow

$\mathrm{t} 1=$ third $*(\operatorname{Tflow}(1,1)+\operatorname{Tflow}(2,2)+\operatorname{Tflow}(3,3))$ !overwrites $\mathrm{t} 1$

! Calculate the deviatoric part of Tflow

$\operatorname{Tflow}(1,1)=\operatorname{Tflow}(1,1)-\mathrm{t} 1$

Tflow $(2,2)=\operatorname{Tflow}(2,2)-\mathrm{t} 1$

$\operatorname{Tflow}(3,3)=\operatorname{Tflow}(3,3)-\mathrm{t} 1$

!pause

! Calculate the Norm of Tflow

call norm(Tflow,ndir,t2) !overwrites t2

!print *, 'norm devTflow', t2

! Calculate the direction of $\operatorname{dev}($ Tflow)

$\mathrm{N}=$ Tflow/t2

!print *, 'N', N

! Calculate the magnitude of $\operatorname{dev}($ Tflow) tau $=\mathrm{t} 2 /$ sqrt (two)

! Perform the inelastic update

$\mathrm{t} 1=$ tau-tau0

$\mathrm{t} 2=\operatorname{detGt}-\mathrm{nu} * \operatorname{det} \mathrm{G} 0$

$\mathrm{t} 3=$ third $^{*}(\operatorname{stressNew}(\mathrm{i}, 1)+\operatorname{stressNew}(\mathrm{i}, 2)+\operatorname{stressNew}(\mathrm{i}, 3))$

\section{If (t1.le.zero) then}

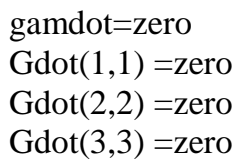

$\operatorname{Gdot}(2,2)=$ zero

$\operatorname{Gdot}(3,3)=$ zero 


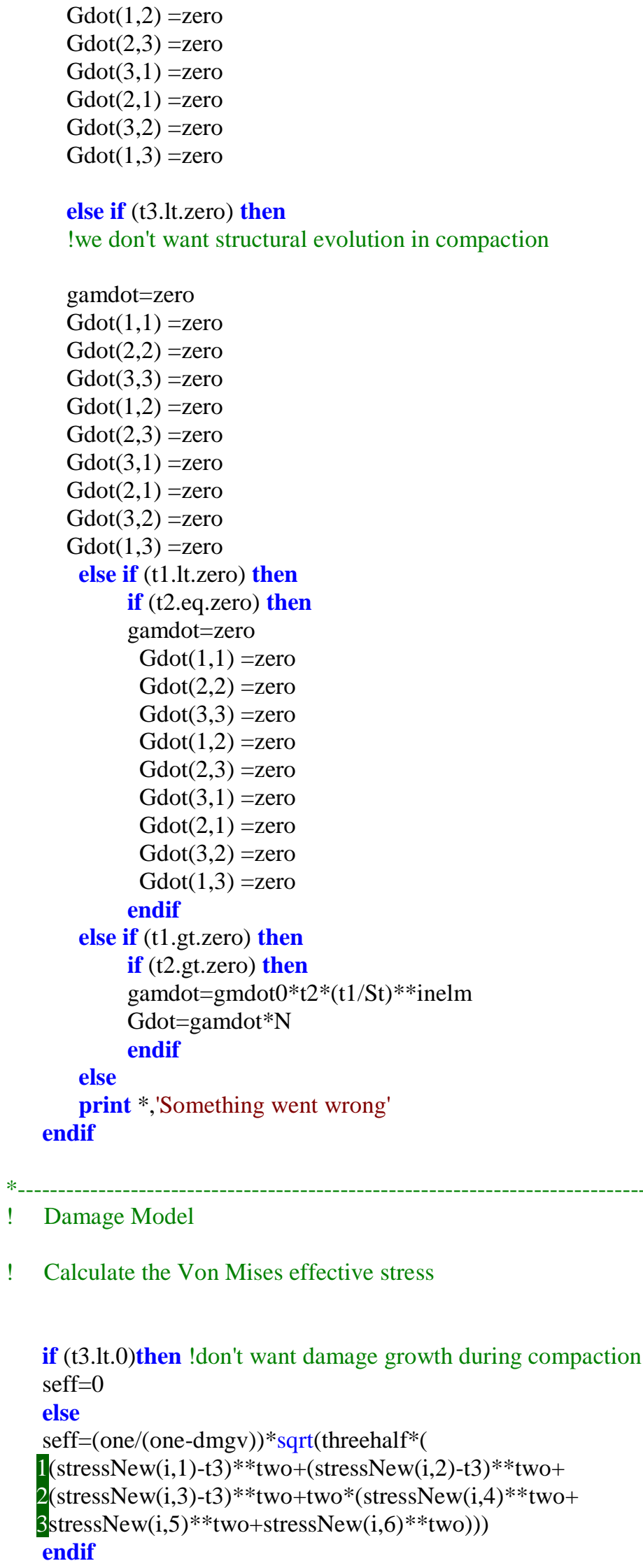

! Calculate the Von Mises effective stress

if ( $\mathrm{t} 3$.lt.0)then !don't want damage growth during compaction seff $=0$

else seff $=($ one $/($ one- $\mathrm{dmgv})) *$ sqrt $($ threehalf* ( 
! Random number generator for stochastic effects

$\mathrm{t} 2=$ one

call srand (20029, oseed,t1)

if (t1.le.half)then

$\mathrm{t} 2=$ one $-5 * \mathrm{t} 1 / 100$

else

$\mathrm{t} 2=\mathrm{one}+5 *(\mathrm{t} 1-$ half $) / 100$

endif

!damage stress ratio

$\mathrm{dsr}=\mathrm{seff} / \operatorname{sigd} 0 * \mathrm{t} 2$

! Check to see if the damage threshold has been exceeded

! If it has, calculate the damage variable

if(dsr.gt.one)then

!calculate the Bazant and Oh factor

$\mathrm{t} 3=\mathrm{mu}^{*}$ beta

$\operatorname{admg}=$ two $* 1 \mathrm{ch} * \operatorname{sigd} 0 * *$ two/

2(two*t $3 *$ Gfrac-lch*sigd $0 *$ two)

!evolve the damage parameter

$\mathrm{t} 3=$ one-exp $(-($ admg $*($ dsr-one $)) * *$ mdam $)$

if(t3.gt.dmg) then !damage cannot decrease

$\mathrm{dmg}=\mathrm{t} 3$

endif

endif

! update the viscous regularized damage

$\mathrm{t} 4=\mathrm{dt} /(\mathrm{etad}+\mathrm{dt})^{*} \mathrm{dmg}+\mathrm{etad} /(\mathrm{etad}+\mathrm{dt}) * \mathrm{dmgv}$

if (t4.gt.dmgv) then !check to ensure dmgv never decreases $\mathrm{dmgv}=\mathrm{t} 4$

endif

! check for total failure

if(dmgv.ge.maxd)then

stateNew $(\mathrm{i}, 25)=$ zero !erode if totally failed

dmgv=one

endif

! ..... Store the new Gt

stateNew $(\mathrm{i}, 1)=\mathrm{Gt}(1,1)$

stateNew $(\mathrm{i}, 2)=\mathrm{Gt}(2,2)$

stateNew $(\mathrm{i}, 3)=\mathrm{Gt}(3,3)$

stateNew $(\mathrm{i}, 4)=\mathrm{Gt}(1,2)$

stateNew $(\mathrm{i}, 5)=\mathrm{Gt}(2,3)$

stateNew $(\mathrm{i}, 6)=\mathrm{Gt}(3,1)$

! ..... Store the new Uinelastic .....

stateNew $(\mathrm{i}, 7)=\mathrm{Ui}(1,1)$

stateNew $(\mathrm{i}, 8)=\mathrm{Ui}(2,2)$

stateNew $(\mathrm{i}, 9)=\mathrm{Ui}(3,3)$

stateNew $(\mathrm{i}, 10)=\mathrm{Ui}(1,2)$ 
stateNew $(\mathrm{i}, 11)=\mathrm{Ui}(2,3)$

stateNew $(\mathrm{i}, 12)=\mathrm{Ui}(3,1)$

! ..... Update St the inelastic hardening variable .....

stateNew $(\mathrm{i}, 13)=\mathrm{St}+\mathrm{alpha} * \mathrm{gamdot} * \mathrm{dt}$

! ..... Store the new Gdot

!print *, 'Gdot end', Gdot

stateNew $(\mathrm{i}, 14)=\operatorname{Gdot}(1,1)$

stateNew $(\mathrm{i}, 15)=\operatorname{Gdot}(2,2)$

stateNew $(\mathrm{i}, 16)=\operatorname{Gdot}(3,3)$

stateNew $(\mathrm{i}, 17)=\operatorname{Gdot}(1,2)$

stateNew $(i, 18)=\operatorname{Gdot}(2,3)$

stateNew $(\mathrm{i}, 19)=\operatorname{Gdot}(3,1)$

! ..... Store the new count value .....

stateNew(i,20)=count

! ..... Store the new damage parameter .....

stateNew $(\mathrm{i}, 21)=\mathrm{dmg}$

! ..... Store the new viscous damage parameter .....

stateNew $(\mathrm{i}, 22)=\mathrm{dmgv}$

!! ..... Store the new seed for the pseudorandom number generator .....

! $\quad$ stateNew $(\mathrm{i}, 23)=$ oseed

100 CONTINUE

endif !1

RETURN

END !end of the VUMAT

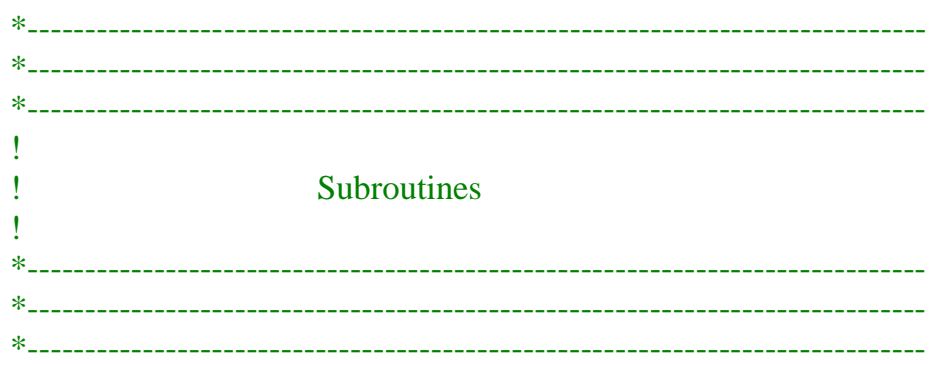

* $1 \quad$ NxN Matrix Push Forward Subroutine "push"

!

! Subroutine to push an nxn matrix forward into the present configuration.

! This could also be called to rotate a matrix forward into the present

! configuration.

! INPUTS:

! 1. M $\rightarrow$ Matrix to be pushed (or rotated) forward

! 2. U $\rightarrow$ Either deformation gradient or the rotation matrix

! 3. ndir $\rightarrow$ Size of the matrix to be pushed (or rotated) forward

! ( $\quad(\mathrm{nxn}==$ ndirxndir)ndir is included in this subroutine 


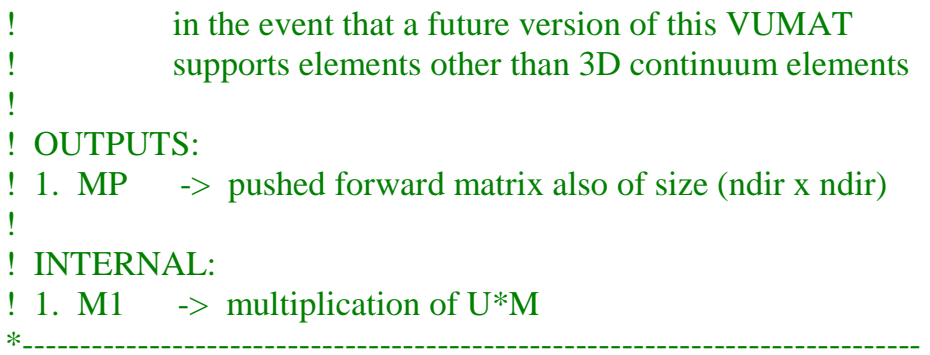

subroutine push(M,U,ndir,Mpush)

integer ndir, $\mathrm{i}, \mathrm{j}$

real $\mathrm{M}$ (ndir,ndir),M1(ndir,ndir),U(ndir,ndir),Ut(ndir,ndir),

1 Mpush(ndir,ndir)

! Multiply U times m

$\mathrm{M} 1=\operatorname{matmul}(\mathrm{U}, \mathrm{M})$

$\mathrm{Ut}=\operatorname{transpose}(\mathrm{U})$ ! if using $\mathrm{U}$ the transpose is unnecessary

! multiply ( $\left.\mathrm{U}^{*} \mathrm{M}\right)$ by $\mathrm{Ut}$ to get $\mathrm{M} 1$

Mpush=matmul(M1,Ut)

return

end

* 2 inverse langevin function subroutine "lnginv"

this subroutine calculates the inverse langevin function of an input argument. this input argument is either $1 / 1$ lock for beta 0 or leff/llock for beta.

this subroutine can use a taylor series approximation of the inverse langevin function, or a Pade' approximant (which leads to a stiffer response with fewer terms than a taylor series approximation). The Pade approximant suffers from an instability as the input argument exceeds a value of one.

inputs:

1. leff $\rightarrow$ effective stretch

2. llock $\rightarrow$ locking stretch

!

! outputs:

! 1. beta $\rightarrow$ output of the inverse langevin function

!

subroutine lnginv(leff,llock, beta)

! argument declarations

real leff,llock, beta, $\mathrm{x}$

parameter thirtyfive $=3.5 \mathrm{e} 1$, thirtythree $=3.3 \mathrm{e} 1$, twelve $=1.2 \mathrm{e} 1$,

1 two $=2 \mathrm{~d} 0$, three $=3 \mathrm{~d} 0$

! calculate the stretch ratio leff/llock

$\mathrm{x}=$ leff/llock

calculate the inverse langevin function

beta $=3 * x+9 / 5 * x * * 3+297 / 175 * x * * 5+1539 / 875 * x * * 7$ !Taylor 


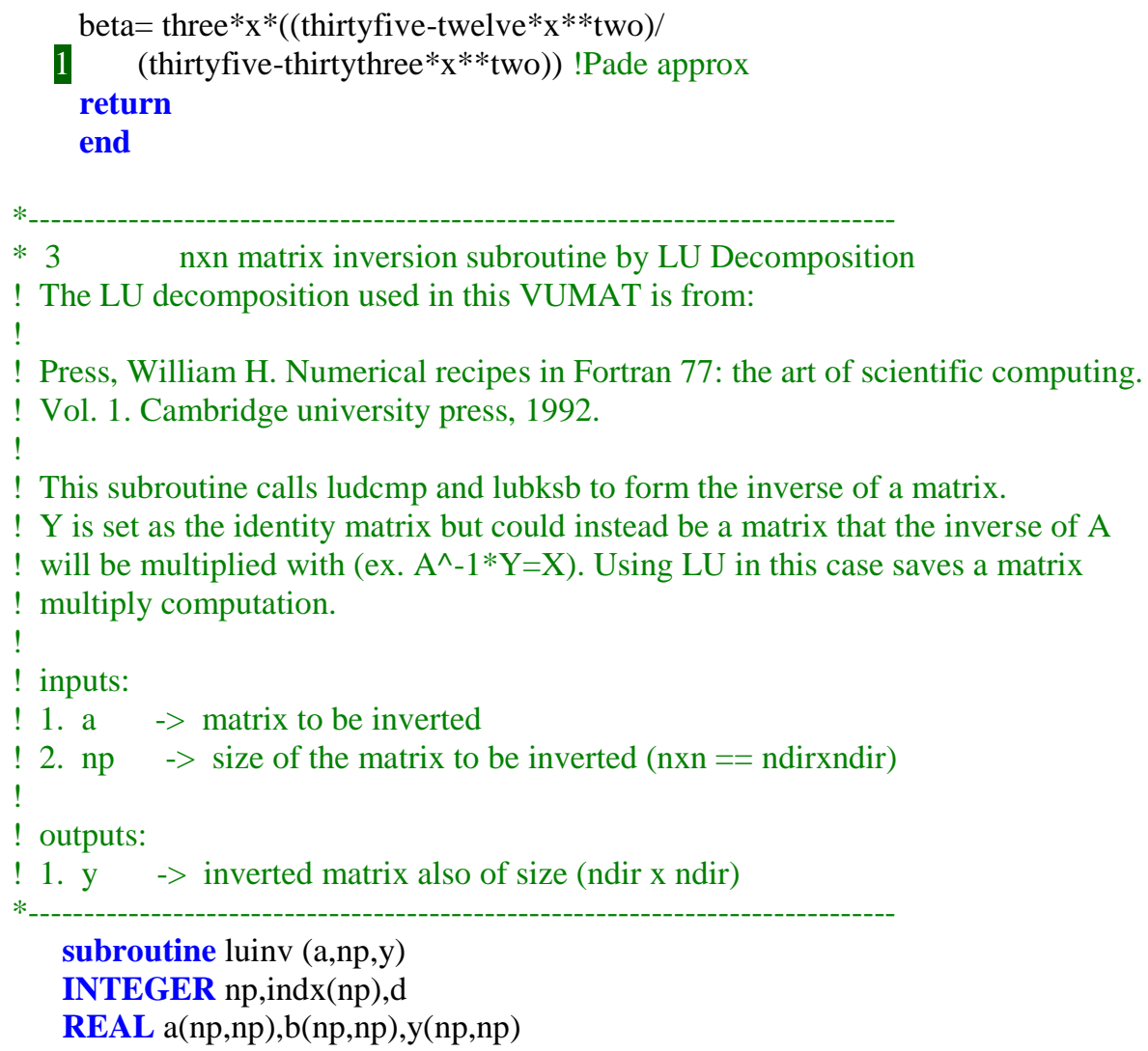

call ludcmp(b,n,np,indx,d)! Decompose the matrix just once.

do $\mathrm{j}=1, \mathrm{n}$ !13 Find inverse by columns.

call lubksb(b,n,np,indx,y $(1, \mathrm{j})$ )

! Note that FORTRAN stores two-dimensional matrices by column, so $\mathrm{y}(1, \mathrm{j})$ is the

! address of the jth column of $y$.

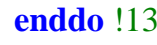

\section{* 4 LU Decomposition of an nxn matrix}

Given a matrix a(1:n,1:n), with physical dimension $n p$ by $n p$, this routine replaces it by the LU decomposition of a rowwise permutation of itself. a and $n$ are input. a is output, arranged as in equation (2.3.14) above; indx(1:n) is an output vector that records the row permutation effected by the partial pivoting; $d$ is output as \pm 1 depending on whether the number of row interchanges was even or odd, respectively. This routine is used in combination with lubksb to solve linear equations or invert a matrix. 


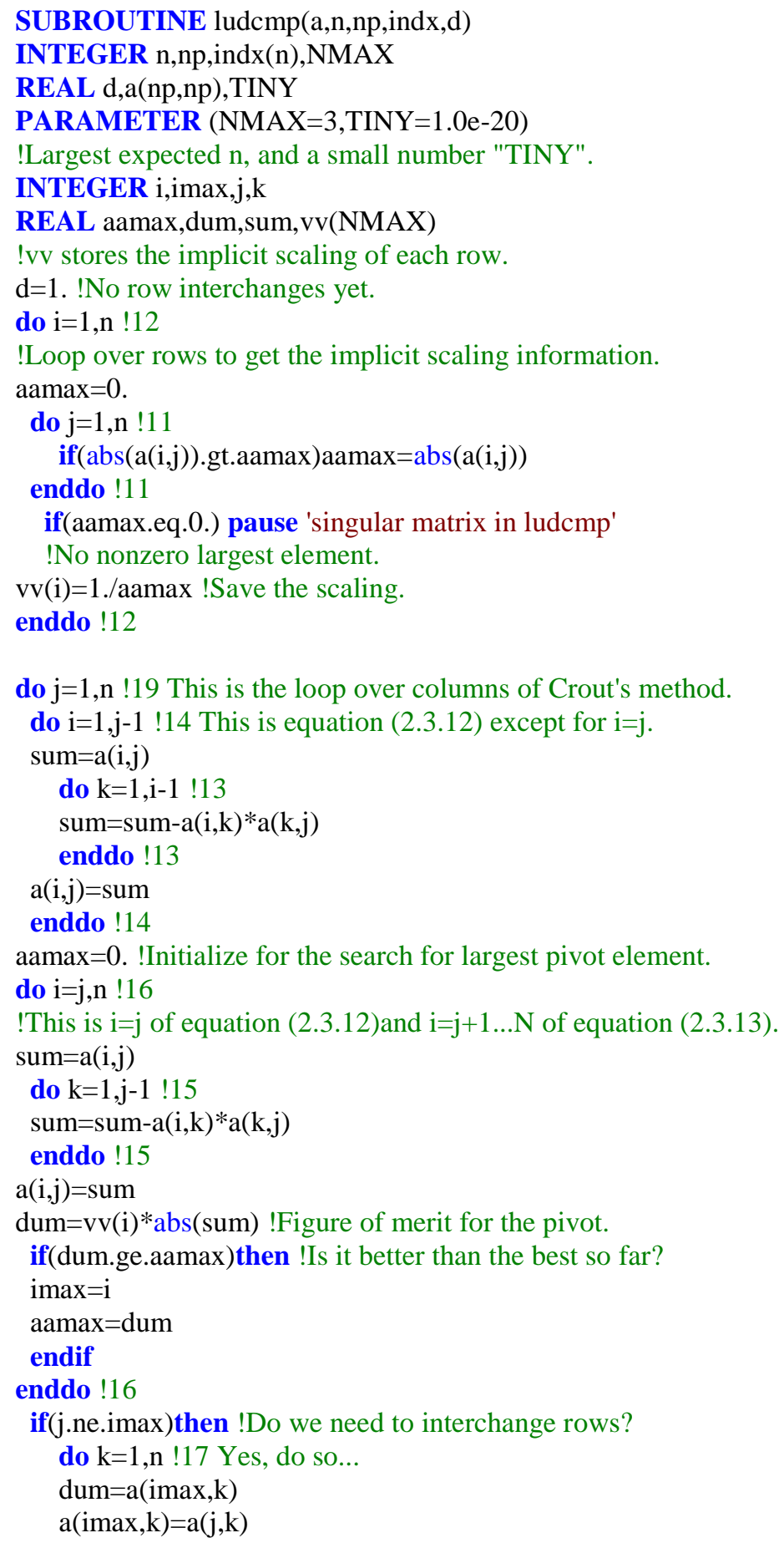


$\mathrm{a}(\mathrm{j}, \mathrm{k})=\mathrm{dum}$

enddo !17

$\mathrm{d}=-\mathrm{d} !$....and change the parity of $\mathrm{d}$.

$\mathrm{vv}(\mathrm{imax})=\mathrm{vv}(\mathrm{j})$ ! Also interchange the scale factor.

endif

$\operatorname{indx}(\mathrm{j})=\mathrm{imax}$

if $(a(j, j) . e q \cdot 0) a.(j, j)=T I N Y$

If the pivot element is zero the matrix is singular

(at least to the precision of the algorithm).

! For some applications on singular matrices, it is desirable to substitute

! TINY for zero.

if(j.ne.n)then !Now, finally, divide by the pivot element.

dum $=1 . / \mathrm{a}(\mathrm{j}, \mathrm{j})$

do $i=j+1, n ! 18$

$a(i, j)=a(i, j) * d u m$

enddo $! 18$

endif

enddo !19 !Go back for the next column in the reduction.

return

END

\section{* 5 LU Back substitution of an nxn matrix}

Solves the set of $\mathrm{n}$ linear equations $\mathrm{A} * \mathrm{X}=\mathrm{B}$. Here $\mathrm{a}$ is input, not as the matrix A but rather as its LU decomposition, determined by the routine ludcmp. indx is input as thepermutation vector returned by ludcmp. $b(1: n)$ is input as the right-hand side vector $\mathrm{B}$,and returns with the solution vector X. a,n,np, and indx are not modified by this routineand can be left in place with successive call of right-hand sides $b$. This routine takes into account the possibility that $\mathrm{b}$ will begin with many zero elements,so it is effiecient for use in matrix inversion.

inputs:

! 1. a $\quad \rightarrow$ decomposed matrix

! 2. $\mathrm{n} \quad \rightarrow$ size of the matrix

! 3. np $\quad \rightarrow$ same as $\mathrm{n}$

! 4. indx $\rightarrow$ permutation vector

! 5. b $\rightarrow$ right hand side vector

!

! outputs:

! 1. b $\quad \rightarrow$ now the $\mathrm{x}$ vector (destroys $\mathrm{b}$ )

SUBROUTINE lubksb(a,n,np,indx,b)

INTEGER $\mathrm{n}, \mathrm{np}, \operatorname{indx}(\mathrm{n})$

REAL a(np,np),b(n)

! Local Declarations

INTEGER $\mathrm{i}, \mathrm{ii}, \mathrm{j}, 11$

REAL sum

ii $=0$

! When ii is set to a positive value, it will become the index

! of the first nonvanishing element of $b$. We now do

! the forward substitution, equation (2.3.6). The only new

! wrinkle is to unscramble the permutation as we go. 


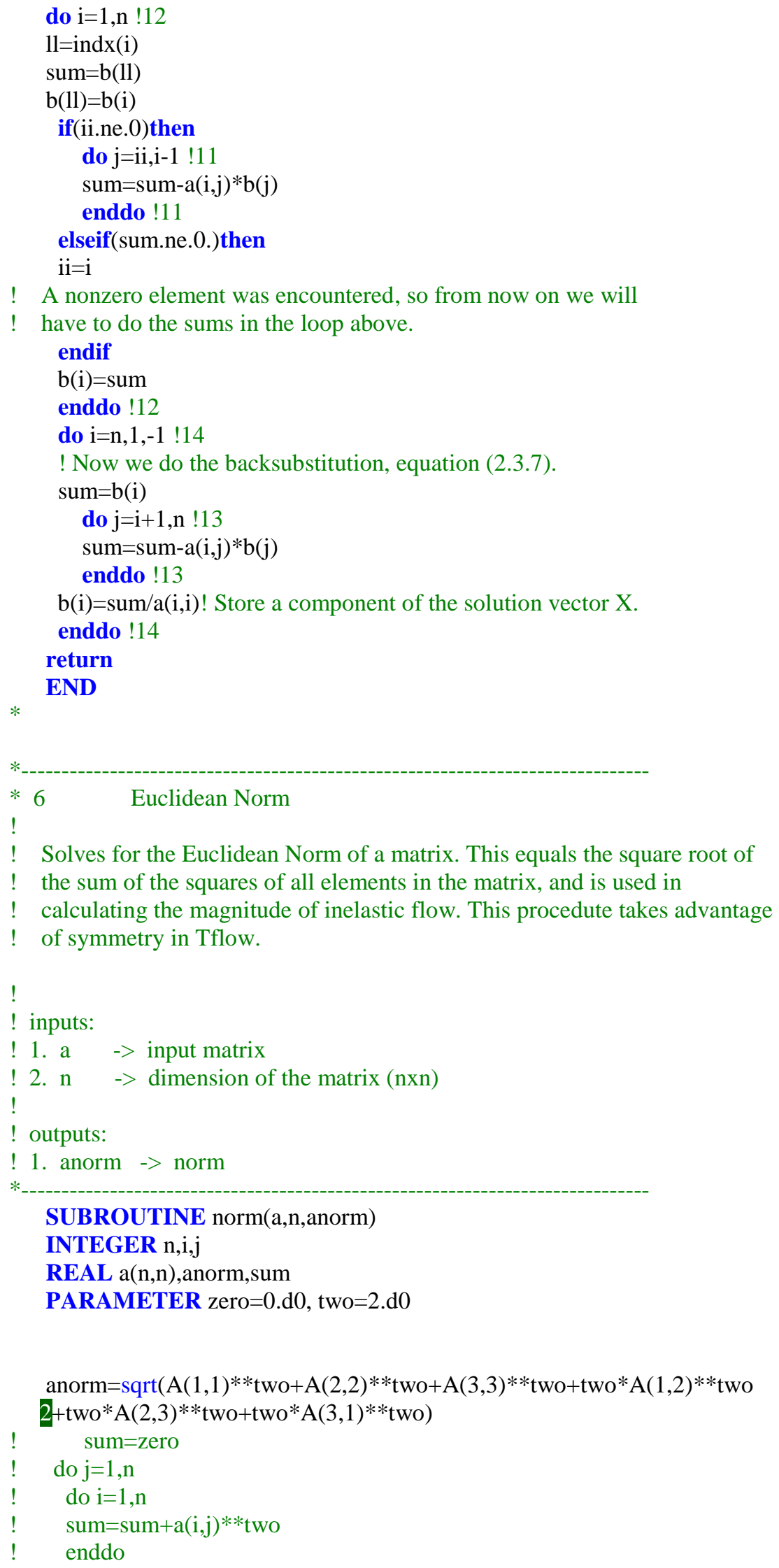




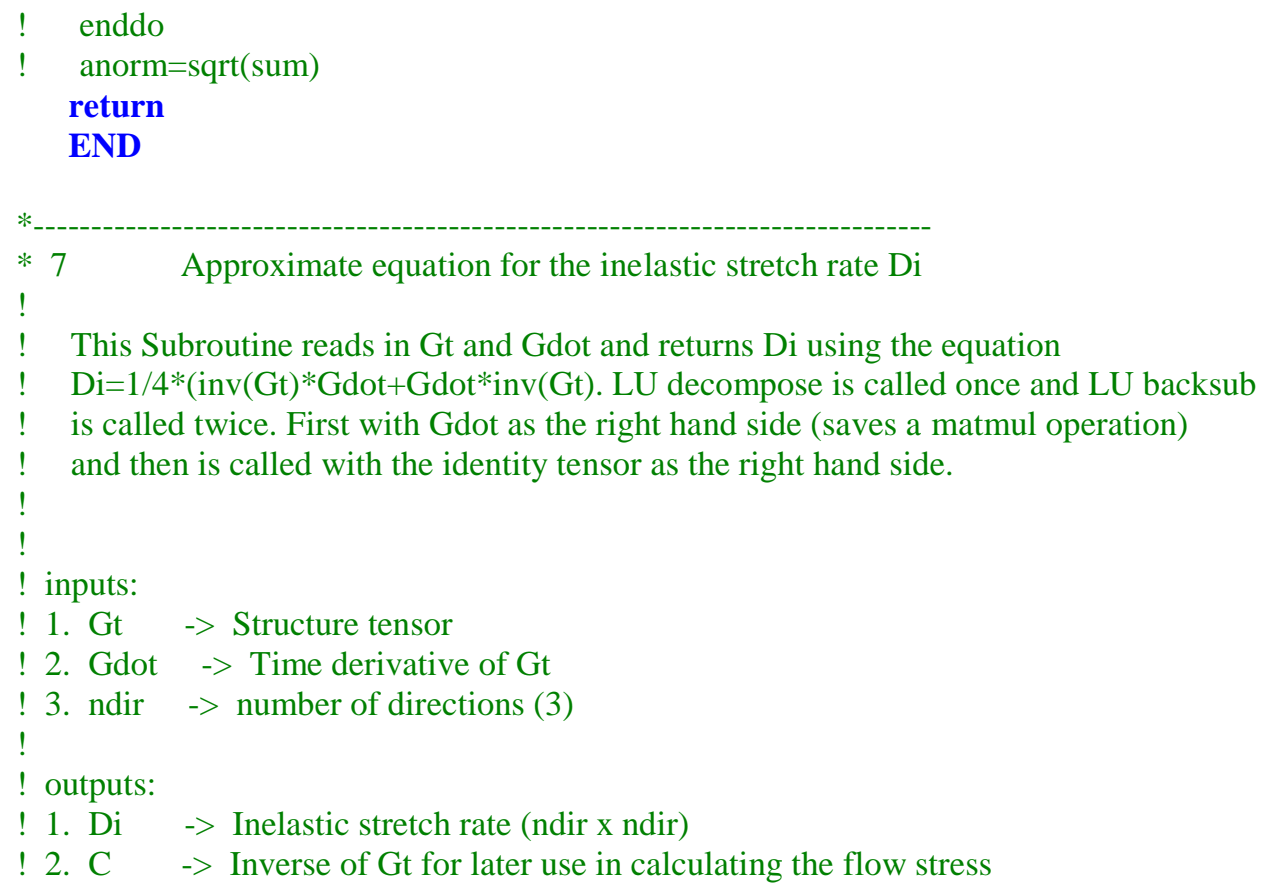


! Calculate Di

$\mathrm{Di}=$ fourth*(B+matmul $($ Gdot, $\mathrm{C}))$

!replace any very small numbers with zero to avoid underflow

do $\mathrm{k}=1$, ndir

do $\mathrm{j}=1$, ndir

if $(\operatorname{abs}(\operatorname{Di}(k, j)) \cdot$ le.tiny) then

$\operatorname{Di}(k, j)=$ zero

endif

if $(\operatorname{abs}(\mathrm{C}(\mathrm{k}, \mathrm{j})) \cdot$ le.tiny $)$ then

$\mathrm{C}(\mathrm{k}, \mathrm{j})=$ zero

endif

enddo

enddo

return

END

* 8 srand

!

! This subroutine calculates a pseudorandom number between 0 and 1 . It can

! be used to model stochastic effects in the damgage growth.

inputs:

! 1. iseed $\rightarrow$ initial seed

!

! outputs:

! 1. random $\rightarrow$ a pseudorandom number between 0 and 1

! 2. oseed $\rightarrow$ the integer seed

subroutine srand(iseed,oseed,random)

Integer iseed,c1,c2, oseed

Parameter $(\mathrm{c} 1=19423, \mathrm{c} 2=811)$

Real random

if (oseed.eq.0) then

oseed $=$ iseed

endif

oseed $=\bmod (\mathrm{c} 1 *$ oseed, $\mathrm{c} 2)$

random=real(oseed)/real(c2)

return

end 